\title{
VARIATIONS IN GENES RELATED TO SLEEP PATTERNS IN CHILDREN WITH AUTISM SPECTRUM DISORDER
}

by

Ann E. E. Johansson

Bachelor of Science in Nursing, Indiana Wesleyan University, 2007

\author{
Submitted to the Graduate Faculty of \\ the School of Nursing in partial fulfillment \\ of the requirements for the degree of \\ Doctor of Philosophy
}

University of Pittsburgh 


\title{
UNIVERSITY OF PITTSBURGH
}

\author{
School of Nursing
}

This dissertation was presented

by

Ann E. E. Johansson

It was defended on

November 30, 2017

and approved by

Bernie Devlin, PhD, Professor, Department of Psychiatry

Christine A. Feeley, PhD, RN Assistant Professor, Health Promotion \& Development

Eileen R. Chasens, PhD, RN, FAAN, Associate Professor, Health \& Community Systems Janice S. Dorman, PhD, MS, Professor, Health Promotion \& Development 
Copyright (C) by Ann E. E. Johansson

2017 


\title{
VARIATIONS IN GENES RELATED TO SLEEP PATTERNS IN CHILDREN WITH AUTISM SPECTRUM DISORDER
}

\author{
Ann E. E. Johansson, PhD, RN \\ University of Pittsburgh, 2017
}

Background: Autism spectrum disorder (ASD) is accompanied by comorbid sleep problems in $40-80 \%$ of cases. Previous reports have shown relationships between circadian rhythm genes and sleep problems in ASD. This study aimed to (1) examine relationships between sleep problems and characteristics of children with ASD—age, sex, ASD severity, IQ, and medical conditions, and (2) explore genetic associations between 25 melatonin synthesis and suprachiasmatic nucleus (SCN) genes and sleep patterns in children with ASD.

Methods: This secondary analysis used genotypic and phenotypic data on children aged 4-18years from the Simons Simplex Collection. An exploratory factor analysis and psychometric evaluation was performed on the Simons Simplex Collection Sleep Interview (SSCSI), consisting of Nighttime problems, Daytime problems, and Sleep duration problems subscales. Expression quantitative trait loci (eQTL) and single nucleotide polymorphisms (SNPs) were identified primarily from gene expression data for 25 melatonin/SCN genes. Student's $t$-tests, Chi-square, and Kruskal-Wallis or Mann-Whitney U tests were performed to detect differences between age groups, sexes, and sleep problem groups. Associations between sleep problems and child characteristics were assessed using logistic regression. Relationships between sleep problems and gene variants were tested with logistic regression and transmission disequilibrium tests.

Results: Approximately $41 \%$ of children were categorized as having sleep problems. In the full sample, and in age subgroups, GID and age were the variables most frequently associated with 
composite sleep problems, Nighttime problems, Daytime problems, and Sleep duration problems, followed by NVIQ and male sex. Seizures were associated with composite sleep problems and Nighttime problems. ASD severity was not associated with sleep problems in this sample. No significant associations were found between sleep problems and any gene variants after correction for multiple testing.

Conclusions: This study advances our understanding of sleep in ASD by showing that GID, NVIQ, age, sex, and seizures increased the odds of sleep problems; but that the gene expressionrelated variants in the melatonin synthesis and SCN pathways do not have a notable impact on sleep problems in children with ASD. These results reinforce that healthcare professionals should screen for sleep problems in children with ASD and suggest future lines of inquiry. 
TABLE OF CONTENTS

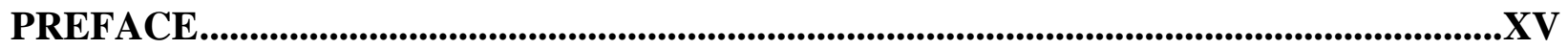

DEFINITIONS OF TERMS ....................................................................................................XIX

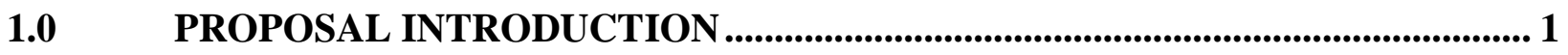

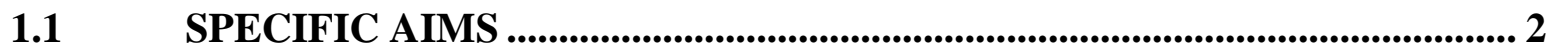

1.1.1 Conceptual models................................................................................................ 3

1.2 SIGNIFICANCE, INNOVATION, AND BACKGROUND ............................ 5

1.2.1 Significance................................................................................................................ 5

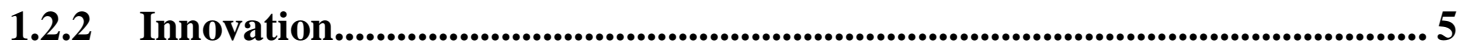

1.2.3 Background ................................................................................................................ 6

1.2.3.1 Sleep and development in the healthy child.......................................... 6

1.2.3.2 Measurement of sleep patterns .................................................................. 12

1.2.3.3 Support for investigation of sleep patterns in autism spectrum disorder 13

1.2.3.4 Comparison of sleep in children with ASD and TD children .......... 14

1.2.3.5 Intellect, ASD severity, and sleep …........................................................ 17

1.2.3.6 Medical conditions and sleep .................................................................... 18

1.2.3.7 Support for investigation of circadian genes and sleep patterns .... 18

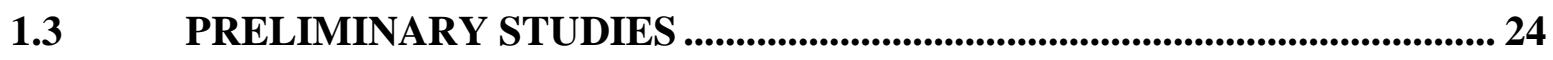


1.3.1 Simons Simplex Collection Sleep Interview ................................................. 24

1.4 RESEARCH DESIGN AND METHODS.................................................. 25

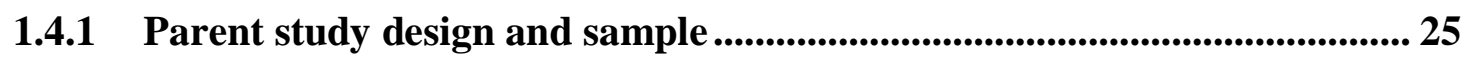

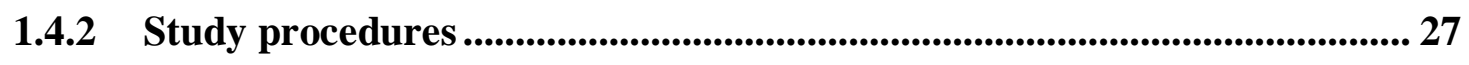

1.4.2.1 Genotype data collection .............................................................. 27

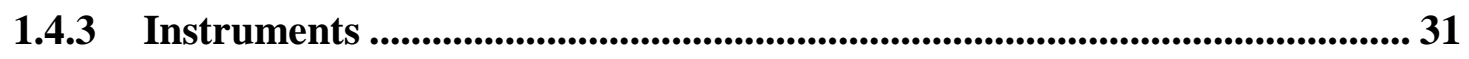

1.4.3.1 Sleep.......................................................................................................... 31

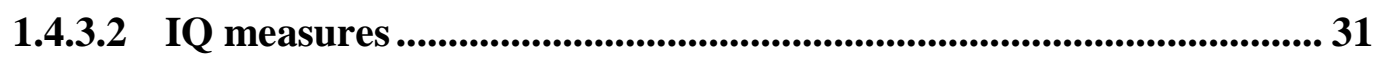

1.4.3.3 ASD severity measures ............................................................. 34

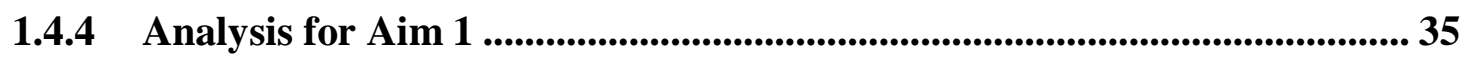

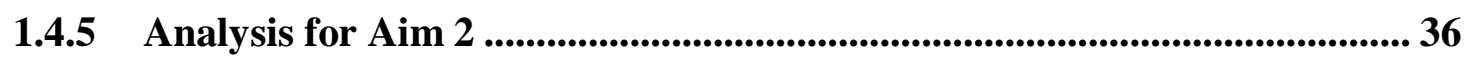

1.4.5.1 Logistic regression analysis ............................................................ 36

1.4.5.2 Transmission disequilibrium test ................................................... 37

1.5 STRENGTHS, POTENTIAL LIMITATIONS, AND ALTERNATE

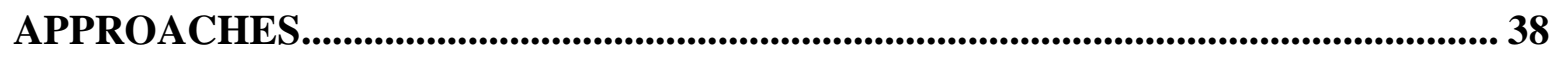

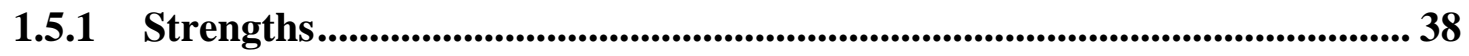

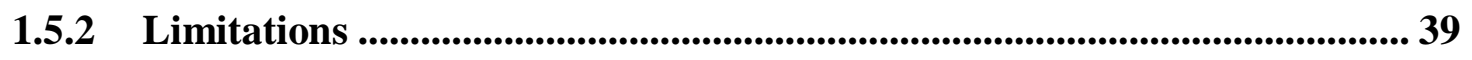

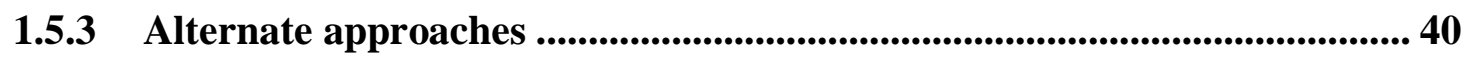

1.6 PROTECTION OF HUMAN SUBJECTS .............................................. 41

1.6.1 Potential risks for proposed secondary analysis......................................... 41

1.6.2 Procedures for protection against risk ................................................... 41

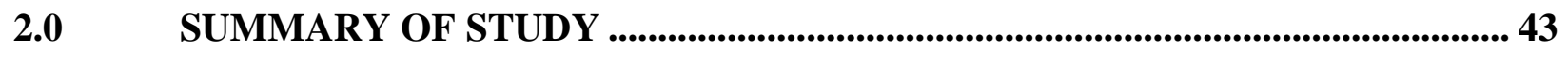

PRELIMINARY WORK ON DISSERTATION STUDY ............................ 43 
$2.2 \quad$ PROPOSAL CHANGES...................................................................................... 43

2.3 CONCLUSIONS, IMPLICATIONS FOR NURSING, AND FUTURE

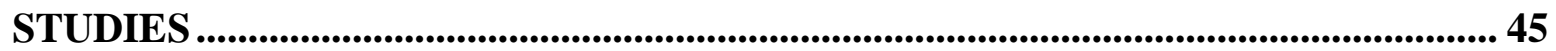

3.0 INSTRUMENTATION MANUSCRIPT: PSYCHOMETRIC PROPERTIES OF

THE SIMONS SIMPLEX COLLECTION SLEEP INTERVIEW ....................................... 48

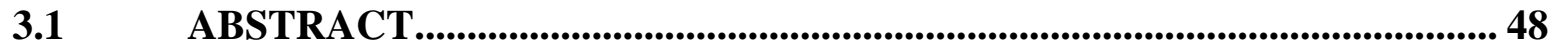

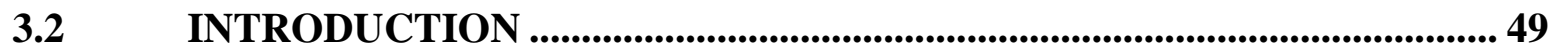

3.2.1 Study aims …......................................................................................................... 51

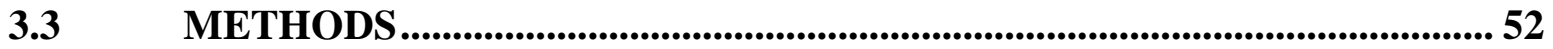

3.3.1 Participants ............................................................................................................ 52

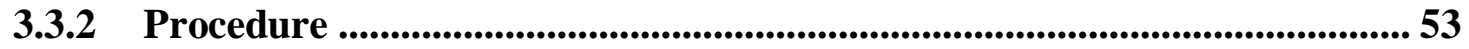

3.3.3 Survey instrument .......................................................................................... 53

3.3.4 Measures................................................................................................................ 54

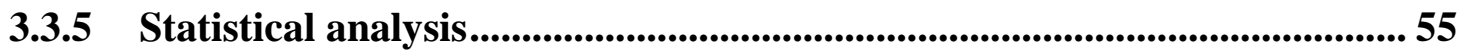

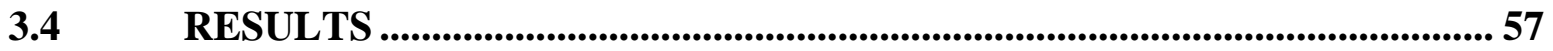

3.4.1 Sample characteristics....................................................................................... 57

3.4.2 Item reduction.............................................................................................5

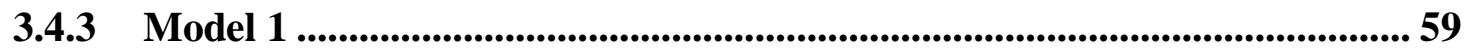

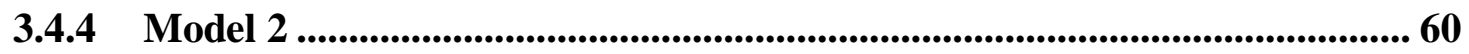

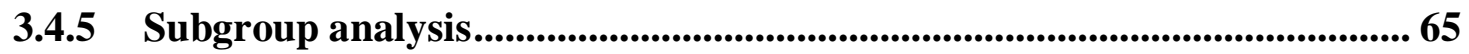

$3.5 \quad$ DISCUSSION

3.5.1 Limitations .......................................................................................................... 67

3.5.2 Implications ............................................................................................................. 68 


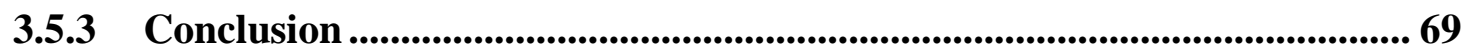

4.0 DATA-BASED MANUSCRIPT: CHARACTERISTICS OF SLEEP IN CHILDREN WITH AUTISM SPECTRUM DISORDER FROM THE SIMONS SIMPLEX

COLLECTION ....................................................................................................................... 70

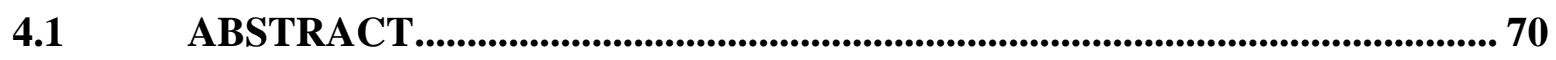

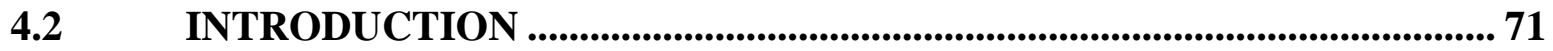

4.2.1 Autism spectrum disorder .............................................................................. 71

4.2.2 Sleep patterns in children with ASD........................................................... 71

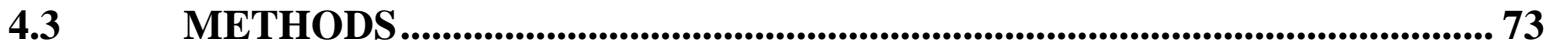

4.3.1 Participants ............................................................................................................ 73

4.3.1.1 Simons Simplex Collection .......................................................................... 73

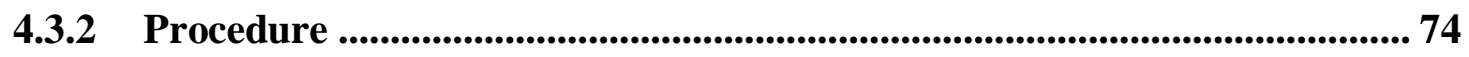

4.3.3 Measures............................................................................................................... 75

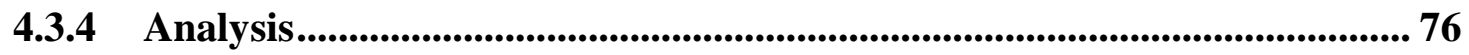

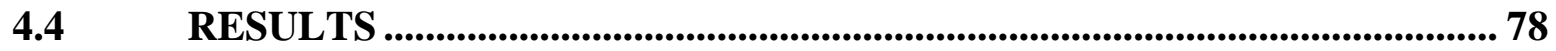

4.4.1 Sample characteristics............................................................................................ 78

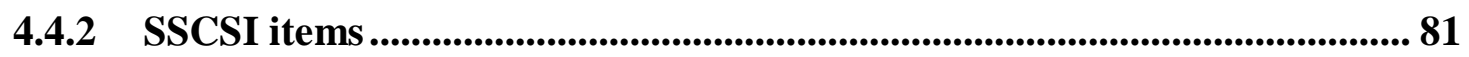

4.4.3 SSCSI composite and subscale scores......................................................... 83

4.4.4 Full sample associations with SSCSI sleep problems .................................... 84

4.4.5 Subgroup associations with SSCSI sleep problems ......................................... 86

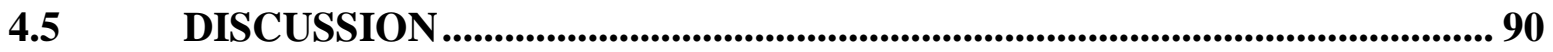

4.5.1 Limitations .............................................................................................................. 94

4.5.2 Implications ............................................................................................................ 95 


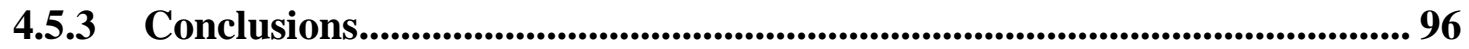

5.0 DATA-BASED MANUSCRIPT: VARIATIONS IN GENES RELATED TO SLEEP PATTERNS IN CHILDREN WITH AUTISM SPECTRUM DISORDERS .......... 97

5.1 ABSTRACT............................................................................................................. 97

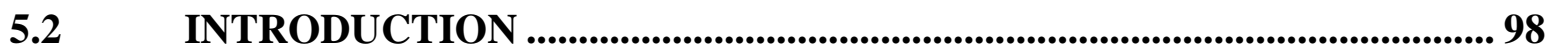

5.2.1 Autism spectrum disorder .............................................................................. 98

5.2.2 Genetics of sleep patterns...................................................................................... 99

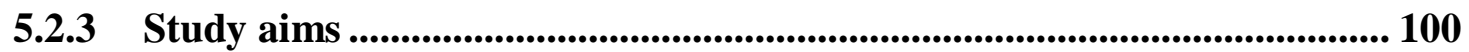

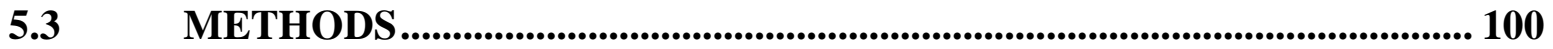

5.3.1 Participants ............................................................................................................. 100

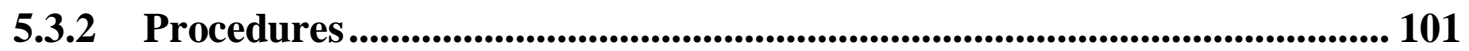

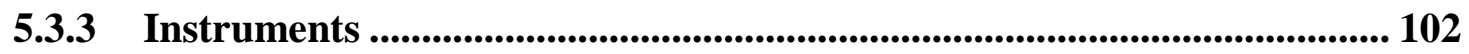

5.3.4 Gene variant selection ...................................................................................... 102

5.3.5 Statistical analyses ......................................................................................... 104

5.3.5.1 Logistic regression analysis ..................................................................... 104

5.3.5.2 Transmission disequilibrium test ........................................................... 105

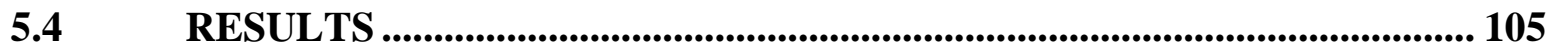

5.4.1 Sample characteristics.......................................................................................... 105

5.4.2 Genotype associations with SSCSI sleep problems ....................................... 107

5.4.3 Transmission disequilibrium test .................................................................. 109

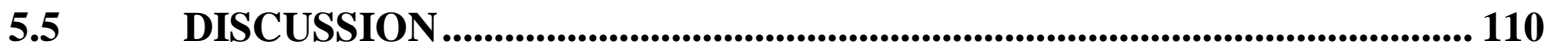

5.5.1 Implications ........................................................................................................... 112

5.5.2 Conclusion .............................................................................................................. 112 
APPENDIX A: COMPARISON OF SLEEP PATTERNS IN CHILDRENWITH ASD AND TYPICALLY DEVELOPING CHILDREN....................................................................... 113

APPENDIX B: MANUSCRIPT \#1 SUPPLEMENTARY TABLES ................................... 120 APPENDIX C: SIMONS SIMPLEX COLLECTION SLEEP INTERVIEW (SSCSI) ..... 123 APPENDIX D: IQ AND AUTISM SEVERITY MEASURE PSYCHOMETRICS............. 125 APPENDIX E: VARIANTS INCLUDED IN GENETIC REGRESSION ANALYSIS ..... 127 APPENDIX F: UNIVERSITY OF PITTSBURGH INSTITUTIONAL REVIEW BOARD

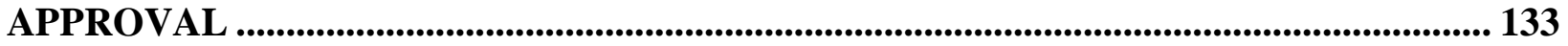
APPENDIX G: LICENSE AGREEMENT FOR MANUSCRIPT \#1 .................................... 135 APPENDIX H: MANUSCRIPT \#2 SUPPLEMENTARY INFORMATION....................... 141 APPENDIX I: TRANSMISSION DISEQUILIBRIUM TEST RESULTS......................... 152

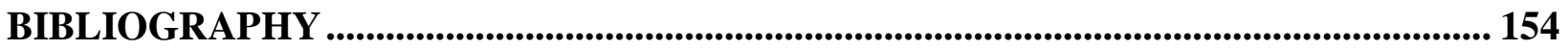




\section{LIST OF TABLES}

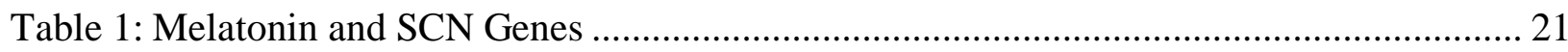

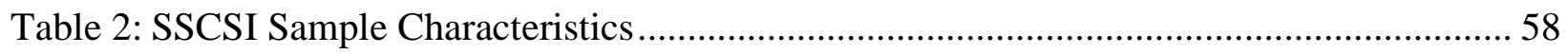

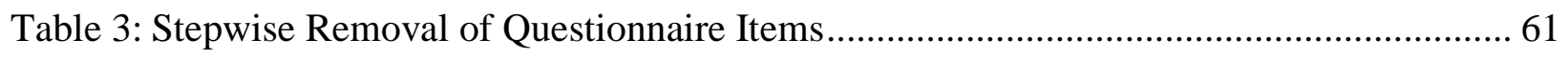

Table 4: Factor Loadings of Principle Components Factor Analysis .............................................. 64

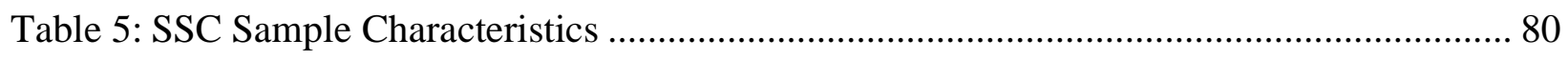

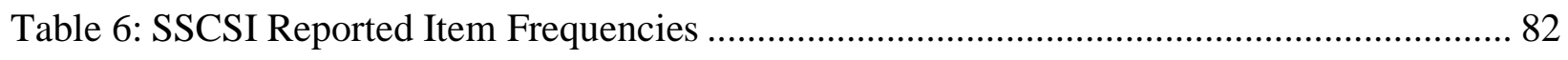

Table 7: SSCSI Composite and Subscale Frequencies Among Children with Sleep Problems... 84

Table 8: Associations Between Child Characteristics and SSCSI Sleep Problems........................ 88

Table 9: Sample Characteristics............................................................................................ 107

Table 10: Genotype Associations with SSCSI Composite and Subscale Sleep Problems ......... 109

Table 11: Comparison of Sleep Patterns in Children with ASD and Typically Developing

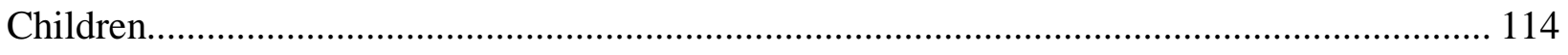

Table 12. Simons Simplex Collection Sleep Interview (SSCSI) ................................................. 124

Table 13: IQ and Autism Severity Measure Psychometrics ....................................................... 126

Table 14: Variants Included in Genetic Regression Analysis .................................................... 128

Table 15: Sample characteristics by SSCSI Sleep Score Categories........................................... 143

Table 16: Reported SSCSI Item Frequencies by Sleep Score Categories .................................... 144

Table 17: SSCSI Composite and Subscale Frequencies by Sleep Score Categories................... 145 
Table 18: Associations Between Child Characteristics and SSCSI Sleep Problems in Early

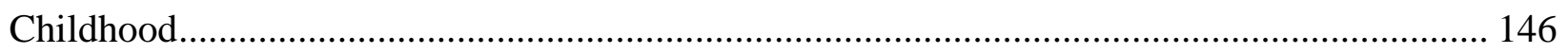

Table 21: Associations Between Child Characteristics and SSCSI Sleep Problems in Middle

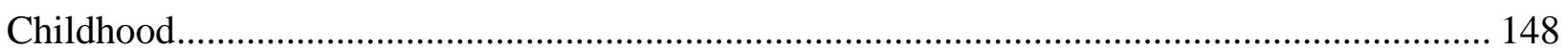

Table 22: Associations Between Child Characteristics and SSCSI Sleep Problems in Late

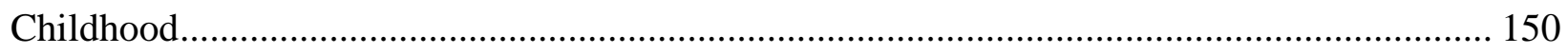

Table 23: Transmission Disequilibrium Test...................................................................... 153 


\section{LIST OF FIGURES}

Figure 1: Aim 1 Conceptual Model .................................................................................... 3

Figure 2: Aim 2 Conceptual Model ............................................................................... 4

Figure 3: Flowchart of Genetic Variant Selection ........................................................... 30

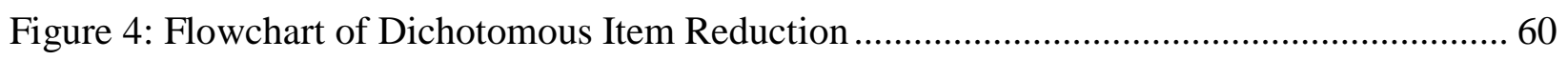

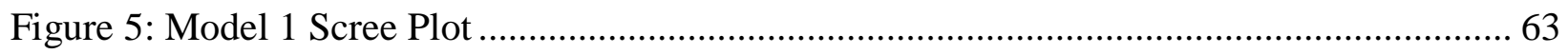

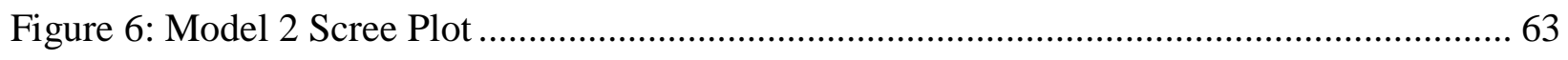

Figure 7: Distribution of SSCSI Item Scores............................................................... 121

Figure 8: Distribution of SSCSI Nighttime Problems Item Scores ..................................... 121

Figure 9: Distribution of SSCSI Daytime Problems Item Scores......................................... 122

Figure 10: Distribution of Sleep Duration Problems Item Scores ......................................... 122

Figure 11: Distribution of SSCSI Composite Sleep Score Categories .................................. 142 


\section{PREFACE}

Ann would like to thank her committee members for sharing their expertise and experience, Dr. Lambertus Klei, and Shawn Wood at the University of Pittsburgh for their analytical assistance on this project, Drs. Benjamin Handen and Vanessa Hus Bal for their consultation on pediatric neuropsychiatry and diagnostic measures, and the Targeted Research and Academic Training Program for Nurses in Genomics (T32NR00975909) and Jayne F. Wiggins Memorial Fund scholarship. Ann would also like to thank her family, friends, and church family for their unending support.

We are grateful to all of the families at the participating Simons Simplex Collection (SSC) sites, as well as the principal investigators (A. Beaudet, R. Bernier, J. Constantino, E. Cook, E. Fombonne, D. Geschwind, R. Goin-Kochel, E. Hanson, D. Grice, A. Klin, D. Ledbetter, C. Lord, C. Martin, D. Martin, R. Maxim, J. Miles, O. Ousley, K. Pelphrey, B. Peterson, J. Piggot, C. Saulnier, M. State, W. Stone, J. Sutcliffe, C. Walsh, Z. Warren, E. Wijsman). We appreciate obtaining access to phenotypic data on the Simons Foundation Autism Research Initiative (SFARI) Base. Approved researchers can obtain the SSC population dataset described in this study (http://sfari.org/resources/sfari-base), by applying at https://base.sfari.org. 


\section{LIST OF ABBREVIATIONS}

ACT $=$ actigraphy

ADI-R = Autism Diagnostic Interview-Revised

ADOS = Autism Diagnostic Observation Schedule

ASD $=$ autism spectrum disorder

$\mathbf{C M C}=$ CommonMind Consortium

CSD = Consensus Sleep Diary

CSHQ = Children’s Sleep Habits Questionnaire

DAS-II = Differential Ability Scales-II

DLPFC = dorsolateral prefrontal cortex

DSPS = delayed sleep phase syndrome

$\mathbf{E E G}=$ electroencephalogram

eQTL = expression quantitative trait loci

FASPS = familial advanced sleep phase syndrome

$\mathbf{F D R}=$ false discovery rate

GABA $=\mathrm{y}$-aminobutyric acid

GID = gastrointestinal distress

GTEx = The Genotype-Tissue Expression Project

GUID = global unique identifiers

GWAS = genome-wide association study

HWE = Hardy Weinberg Equilibrium 


$$
\begin{aligned}
& \text { ID = intellectual disability } \\
& \text { IQ = intelligence quotient } \\
& \text { IRB = Institutional Review Board } \\
& \mathbf{L D}=\text { linkage disequilibrium } \\
& \mathbf{M b}=\text { megabase }
\end{aligned}
$$

MSEL = Mullen Scales of Early Learning

NREM = non-rapid eye movement

NVIQ = nonverbal intelligence quotient

$\mathbf{O R}=$ odds ratio

PSG = polysomnography

$\mathbf{R E M}=$ rapid eye movement

RNA-Seq $=$ RNA sequence

$\mathbf{R} \mathbf{R}=$ risk ratio

$\mathbf{R R B}=$ restricted repetitive behavior

SA $=$ social affect

SCN = suprachiasmatic nucleus

SDB = sleep disordered breathing

$\mathbf{S E}=$ sleep efficiency

SFARI = Simons Foundation Autism Research Initiative

SOL = sleep onset latency

SNP = single nucleotide polymorphism

SSC $=$ Simons Simplex Collection

SSCSI = Simons Simplex Collection Sleep Interview 
SWS = slow wave sleep

TD = typically developing

TST $=$ total sleep time

WASI $=$ Weschler Abbreviated Scale of Intelligence

WASO = wake after sleep onset

WISC-IV = Weschler Intelligence Scale for Children-Fourth Edition 


\section{DEFINITIONS OF TERMS}

Proband: individual under study, often the first individual affected in a family who is brought to medical attention

Simplex family: family in which only one member has the condition under study; in this study, a family in which the proband is the only member of the family with ASD up to third degree relatives affected with ASD

Sleep efficiency: portion of sleep episode taken up by sleep (total sleep time/time in bed)

Sleep latency: period of time from beginning to try to fall asleep to onset of sleep; also known as sleep onset latency

Sleep quality: subjective satisfaction with one’s sleep, including falling asleep, staying asleep, length of sleep, and refreshment on waking

Time in bed: time one spends in bed, from laying down to rising for the final time

Total sleep time: amount of time asleep during a sleep episode; equal to duration of sleep episode minus time awake

Wake after sleep onset: awakenings after initially falling asleep in which the sleeper returns to a relatively alert state 


\subsection{PROPOSAL INTRODUCTION}

Autism spectrum disorder (ASD) is a pervasive developmental disorder characterized by deficits in social communication, and restricted, repetitive interests and behaviors (American Psychiatric Association, 2013). ASD presents early in life, persisting through the lifespan, and is termed a spectrum because symptoms of the disorder vary widely in severity. In addition to core communication and behavior difficulties, children with ASD may suffer from a range of other medical and behavioral comorbidities, such as anxiety, gastrointestinal disorders, seizures, and sleep problems. Sleep problems are estimated to affect $40-80 \%$ of children with ASD, and include difficulty falling asleep (Goldman et al., 2017) and staying asleep (Souders et al., 2009), shorter total sleep time (sleep beginning to end- awakenings after sleep onset; Elrod \& Hood, 2015), poorer sleep efficiency (total sleep time/time in bed; Fletcher et al., 2016; Giannotti et al., 2011). Several studies have also suggested that intellectual ability (Mazurek \& Petroski, 2015; Veatch et al., 2017) and ASD severity may contribute to sleep problems in children with ASD (Zachor \& Ben-Itzchak, 2016). However, the studies of sleep problems in ASD that have been performed thus far have used relatively small samples in a restricted age range, examined a single or select few sleep problems, or did not account for variables such as IQ, ASD severity, or medical problems.

Recent studies have suggested that disrupted levels of melatonin and/or alterations of genes in the suprachiasmatic nucleus, two components of circadian rhythm regulation, may play a role in sleep problems in ASD. Using data from the Simons Simplex Collection (SSC), a large, national ASD consortium genome-wide association study (GWAS) that collected information about sleep patterns, intellectual abilities, ASD symptoms, and medical history, the proposed study seeks to fill these gaps in 
knowledge by (1) examining associations between nighttime and daytime sleep problems and sleep duration problems and characteristics of children with ASD (sex, age, IQ, ASD severity, and medical conditions), and (2) relate variation in and around 25 melatonin synthesis and SCN genes to their influence on sleep problems in children with ASD. Genetic variants have been chosen for association analysis based on their impact on gene expression, as measured by RNA-sequencing (RNASeq) and single nucleotide polymorphism (SNP) data. Sleep patterns to be examined include difficulty going to bed and falling asleep, frequent/prolonged night wakings, difficult morning waking, long/frequent naps, excess daytime sleepiness, irregular bedtime and waketime, and irregular sleep duration. This study helped to distinguish subgroups in ASD; address the question of whether and to what extent IQ, ASD severity, medical conditions, and melatonin-related genes impact sleep patterns; and eventually may even contribute to the search for targeted therapies to improve the quality of life, health, and treatment efficacy of children and families affected by ASD.

\subsection{SPECIFIC AIMS}

Specific Aim 1: Examine the relationship between sleep patterns and characteristics of children with ASD—age, sex, ASD severity, IQ, and medical conditions. We hypothesize that IQ, more severe ASD symptoms, and presence of medical conditions will increase the likelihood of a child having certain sleep patterns.

Specific Aim 2: Explore genetic associations between 25 melatonin synthesis and SCN genes and sleep patterns in children with ASD. We hypothesize that relationships will exist between sleep patterns and variants in the melatonin/SCN genes. 


\subsubsection{Conceptual models}

The conceptual model for aim 1 (Figure 1) demonstrates relationships between child characteristics, measured by the Autism Diagnostic Observation Schedule, four well-validated intelligence tests, and the SSC medical history; and sleep patterns, measured by the Simons Simplex Collection Sleep Interview.

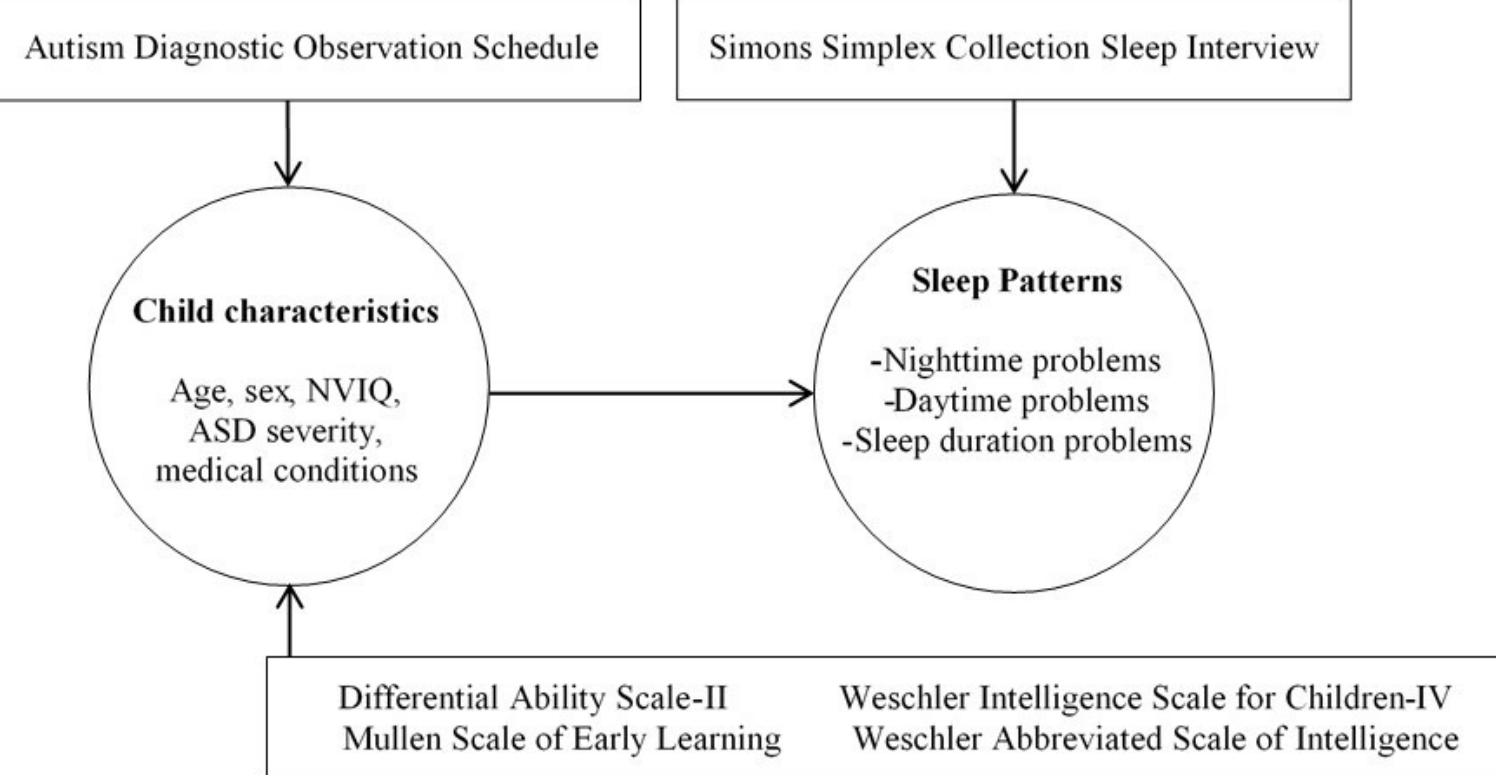

Figure 1: Aim 1 Conceptual Model 
The conceptual model for aim 2 (Figure 2) demonstrates relationships between 25 genes related to melatonin synthesis and SCN circadian function, measured by genotypes from a SNP genotyping array that covered the genome; and sleep patterns, measured by the Simons Simplex Collection Sleep Interview; controlling for child characteristics, measured by the Autism Diagnostic Observation Schedule, four intelligence tests, and the SSC medical history. The model also depicts the interaction between the pineal gland and SCN.

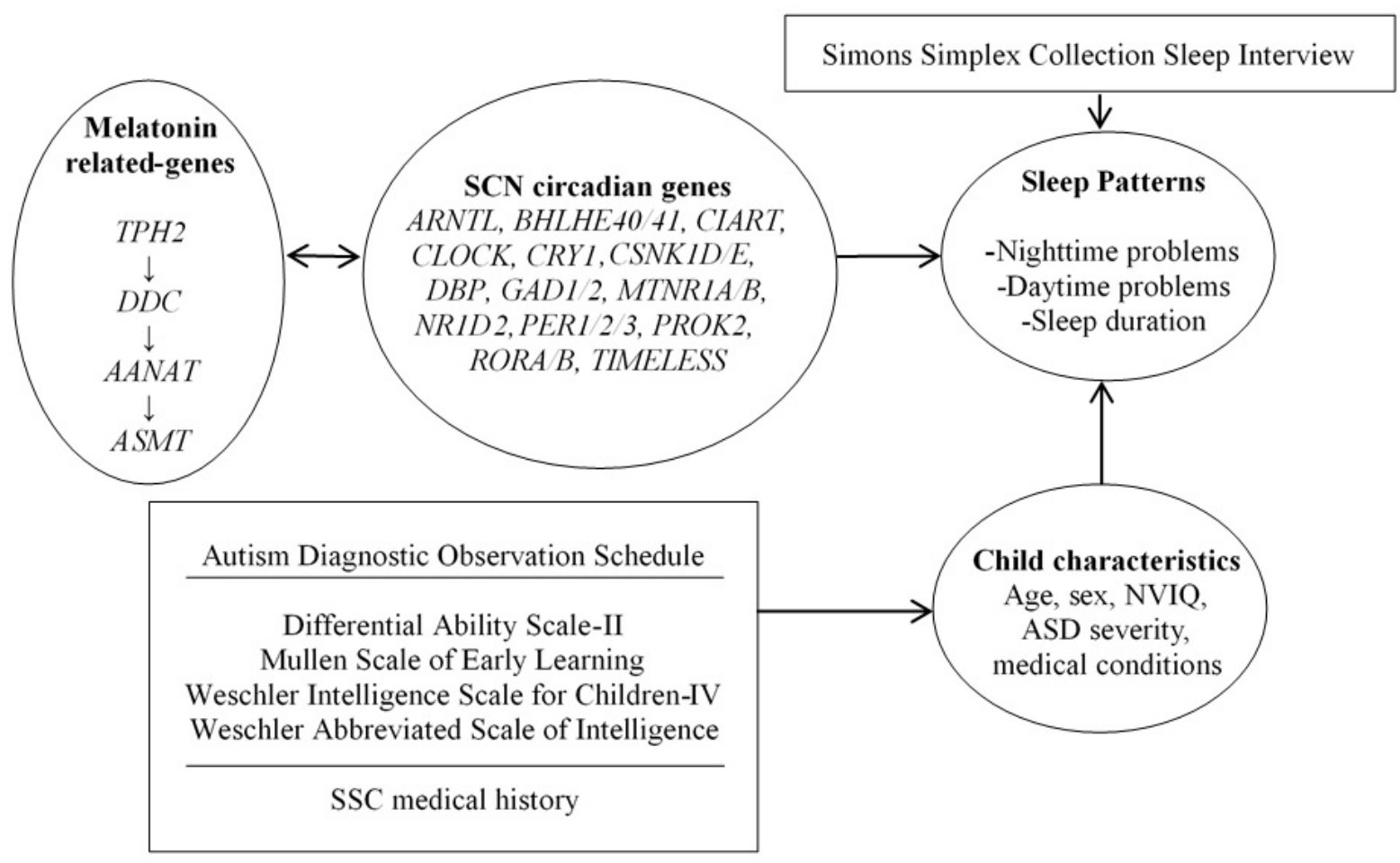

Figure 2: Aim 2 Conceptual Model 


\subsection{SIGNIFICANCE, INNOVATION, AND BACKGROUND}

\subsubsection{Significance}

Roughly half of children diagnosed with ASD have abnormal sleep patterns (Liu, Hubbard, Fabes, \& Adam, 2006; Malow et al., 2006; Souders et al., 2009), which are worsened in children with severe ASD and/or low IQ (DeVincent, Gadow, Delosh, \& Geller, 2007; Gail Williams, Sears, \& Allard, 2004; Schreck, Mulick, \& Smith, 2004; Veatch et al., 2017; Zachor \& Ben-Itzchak, 2016). Adequate sleep is vital for all children for optimal physical and mental health, growth, learning and memory, and peak academic performance. The proposed study aims to describe sleep patterns within the context of ASD, and to determine whether these patterns can be ascribed, in part, to genotypes of children with ASD. The study lays the groundwork to understand from a biological perspective why and how sleep patterns are abnormal in children with ASD. With this firm foundation, it should be possible to build further studies and interventions aimed at making sleep patterns more typical, thereby improving the quality of life for individuals with ASD and their families. Insofar as we are aware, this will be the largest study to statistically characterize patterns of sleep in ASD, and the relationship of those patterns to attributes of ASD subjects. We anticipate that this study will be of keen interest to the field, could form the foundation to understand why these patterns emerge, and will be the essential first step toward understanding the biology of abnormal sleep patterns in ASD.

\subsubsection{Innovation}

The proposed study is innovative in several ways: (1) previous research has not sufficiently explored how biological factors, some affected by genetic variation, influence sleep patterns in ASD. (2) The study takes advantage of a large sample of strictly phenotyped children, from whom data on sleep patterns and genome-wide data are available, to examine an entirely new question. (3) Genetic variants have been 
chosen for association analysis based on their impact on gene expression in the brain, as measured by RNA-Seq and SNP data, providing a closer link to SCN gene expression than other methods for SNP selection. (4) Findings could help to distinguish genetic subgroups within ASD and contribute to sleep research in other closely related neuropsychiatric disorders.

\subsubsection{Background}

\subsubsection{Sleep and development in the healthy child}

Adequate sleep quantity and quality are essential for healthy growth and development from birth through adolescence (Carskadon, 2002; Dahl \& Lewin, 2002; Hobson, 1989; Sadeh, Mindell, \& Rivera, 2011), and for good health and functioning throughout the lifespan. Most researchers now agree that sleep is an active neurologic process in which chemical and hormone levels are restored (Carter, 2005), epigenetic changes occur, psychological homeostasis is restored (Agnati et al., 2011), memories are consolidated and reorganized (Diekelmann, Wilhelm, Wagner, \& Born, 2013), and growth and maturation take place (Carskadon, 2002; Dahl \& Lewin, 2002). Children and adolescents also need to have satisfactory sleep quantity and quality to convert new learning to long-term memory (Diekelmann et al., 2013).

Healthy sleep is comprised of two physiologically distinct phases, non-rapid eye movement (NREM) and rapid eye movement (REM) sleep, that cyclically alternate throughout the sleep period. NREM is made up of four progressively deeper stages, ranging from stage 1 when the sleeper may be easily awakened and may not realize they had fallen asleep, to stages 3 and 4, referred to as slow wave sleep (SWS) because of characteristically slow delta waves. During NREM, the body has low muscle tone and negligible psychological activity. REM sleep is the stage in which individuals dream, and is distinguished by dyssynchronous electroencephalogram (EEG) waves and atonic muscles. The first REM stage of sleep typically occurs 80 to 100 minutes after sleep onset. Over the sleep period, SWS episodes become progressively shorter and REM episodes progressively longer. Sleep quantity and quality may be affected by homeostatic sleep need, circadian phase, environmental temperature and light, medications, 
and sleep or medical disorders (Carskadon \& Dement, 2017).

The two-process model is a biologic conceptual model commonly used to describe the components of the circadian clock that interact to regulate sleep propensity. Sleep homeostasis, or Process S, represents sleep need or sleep debt, and is dependent on previous amounts of sleep and wake time. This process, also referred to as sleep pressure, increases during wakefulness and decreases during sleep. When Process S nears the lower end of its range, it triggers awakening; and when it approaches the upper end of its range, it triggers sleep. Circadian rhythm, or Process C, is controlled by the SCN and is independent of previous sleep or wakefulness. The Process $\mathrm{C}$ alertness signal has a regular diurnal rhythm with a peak in the early evening, and a trough in the early morning. These two processes antagonize each other to produce a balanced sleep/wake propensity (Borbély, Daan, Wirz-Justice, \& Deboer, 2016), and may also be responsible for the cyclic alternation of REM and NREM sleep stages. The rate at which sleep propensity builds up changes across childhood, with the greatest change around puberty.

The structure and characteristics of sleep patterns change considerably from birth to adolescence, and a number of sleep problems may be seen in different age groups. From the fetal period through adolescence, sleep evolves to meet particular needs during developmental processes including memory consolidation (Stickgold, 2005), synaptic growth and pruning (Tononi \& Cirelli, 2006), and formation of white matter connectivity (Yoo, Gujar, Hu, Jolesz, \& Walker, 2007).

\section{Fetuses and infants}

A recognizable pattern of activity and quiet behavior can be seen in fetuses as early as 20 weeks gestation. Newborns (birth to 1-month) sleep about 70\% of a 24-hour period, or approximately 16.8 hours. Sleep in newborns occurs in multiple periods throughout the day and night, not cued by circadian indicators like light and dark. Sleep periods tend to be around 4-hours long, with 1-2 hour wake periods in between. Infant (1- to 12-months) sleep is comprised of two stages, quiet sleep characterized by relaxed muscles and even breathing; and active sleep, characterized by muscle twitches and flickering eyelids. As infants age, quiet sleep will differentiate into the stages of NREM sleep, and active sleep will become 
REM sleep. In the neonatal period, active sleep makes up more of total sleep time than quiet sleep, but quiet sleep increases in length until it predominates by around 3-months-old. In contrast to newborns, who generally enter sleep through active or REM, infants around 4-months begin transitioning to entering sleep predominantly through quiet or NREM sleep. Sleep onset latency is typically less than 15 minutes. By 3-4 months old, a diurnal pattern of sleep and wake can be seen in infants' activity, and recognizable NREM sleep stages begin to appear. Consolidation of sleep into nighttime sleep and daytime naps, and entrainment of sleep to environmental cues develops during the first 6-months of life. The age at which an infant can sleep through the night varies widely and is largely determined by a child's temperament (Mindell, Leichman, \& Composto, 2016; Sheldon, 2014). Recommended sleep time for newborns is between 14- and 17-hours per 24-hour period, and for infants is between 12- and 15-hours per 24-hour period (Hirshkowitz et al., 2015).

A major sleep-related task in infancy is learning to self-soothe, or the ability to fall back to sleep independently. Infants placed in their crib drowsy but awake tend to be better and self-soothing and falling asleep by themselves. Since sleep onset latency lengthens as children age and nighttime wakings continue to be common through the toddler years, the ability to self-soothe becomes more important for parents (Mindell, Sadeh, Kohyama, \& How, 2010).

\section{Toddlers}

Compared with infants, sleep pattern changes in toddlers occur steadily, but more gradually. As children move into the toddler years (1-3 years), sleep continues to consolidate into one long period at night and one or two naps during the day (Mindell et al., 2016). Sleep onset latency increases to 15-30 minutes as toddlers age. At the beginning of the first year, REM periods tend to be uniform in length and spaced evenly throughout the night. As the child ages, REM periods become shorter at the beginning of the night and gradually longer throughout the sleep period. Slow wave sleep periods exhibit the opposite trend, longer in the early night and shorter as the night proceeds (Sheldon, 2014). The recommended sleep duration for toddlers is 11-14 hours per 24 hour period (Hirshkowitz et al., 2015). 
A number of behavioral sleep problems may emerge in the toddler years as regular sleep routines are being established. Two common and related problems are bedtime resistance and separation anxiety. Children may fight going to bed because of fear of being separated from caregivers, or as children develop independence and begin testing their limits. Bedtime resistance may manifest as refusing to lay down in bed and stay in bed, or frequently calling out after they have been put to bed. Another common problem seen in toddlers is nighttime wakings, reported in $25-50 \%$ of parents. These may be due long naps, delayed bedtime, not enough sleep overall, sleep disordered breathing (snoring, sleep apnea), or a sleep environment not conducive to sleep (noise, light, temperature). Although wakings throughout the night are normal at all ages, they become a problem when the child is not able to go back to sleep independently. These three common problems may be helped by a regular bedtime routine and consistent limit setting (Mindell \& Moore, 2014).

\section{Pre-schoolers}

Sleep patterns in preschool-aged children (3-5-years) steadily continue toward mature, adult patterns. A distinct process occurring in this period is a decline in the percentage of REM sleep to total sleep time from about $30 \%$ in toddlers to a more adult level of $20-25 \%$. The age at which naps are given

up varies among children but is most often by 3- to 4-years-old, especially as children enter school. A strict bedtime routine is particularly important when preschoolers are attending school because they need to wake up in time for school while still needing 10-13 hours of sleep per night (Licis, 2018).

The preschool age period is the peak time for sleep disordered breathing (SDB) to emerge. Including obstructive sleep apnea syndrome, SDB ranges from snoring to periodic obstruction of the airway, and has been identified as a risk factor for neurocognitive and behavioral problems in children (Biggs et al., 2014). SDB may be indicated by other symptoms such as enuresis (Su et al., 2011), daytime hyperactivity or excess sleepiness (Ren \& Qiu, 2014), and decreased physical, emotional, and social functioning (Jackman et al., 2013). The majority of SDB cases in children are due to adenotonsillar hypertrophy, treated most often with adenotonsillectomy (Mitchell, Pereira, \& Friedman, 2006). 
Other behavioral sleep problems that may occur in preschool-aged children include "curtain calls” and parasomnias_sleep walking, confusion arousals, or sleep terrors. Curtain calls are repeatedly calling for a caregiver to come back into the room or the child repeatedly leaving the bedroom. Children are usually seeking reassurance that they are safe and a caregiver is nearby. While children are still learning the difference between reality and imagination, children may need to have a caregiver look in closets, under the bed, and in the toy box to assure the child that they are safe. Parasomnias, also known as disorders of arousal, are a group of disorders that involve abnormal or unusual behavior during sleep. A child having a parasomnia may display misperception of or unresponsiveness to the environment, difficulty being aroused, autonomic arousal, automatic behavior, and amnesia to the event after waking. Parasomnias usually occur while transitioning out of slow wave sleep, typically 60-90 minutes after falling asleep, and may last less than one minute to more than 30 minutes. Sleep terrors are typically seen in older children and adolescents. The majority of children naturally grow out of parasomnias (Rosen, 2014). Enuresis in the absence of SDB is common in preschool children while stable bladder control is still being established. In a study of over 16,000 children, an estimated $16 \%$ of 5 -year-old children experienced one episode of enuresis over three-months. Boys were found to be three times more likely to experience enuresis than girls (Yeung, Sreedhar, Sihoe, Sit, \& Lau, 2006).

\section{School-aged children}

Sleep patterns in school age children (6-12-years) resemble that of adults, with the exception of sleep duration. The recommended sleep duration for school age children is 9-11 hours per night, while adults are advised to sleep 7-9 hours per night. The additional time these children sleep is distributed evenly across sleep stages, so that each stage is slightly longer but proportion of each sleep stage to total sleep resembles adults. Distinctively, prepubertal school aged children have the most energy and alertness they have had or will have in their life. Because of this, most school-aged children do not nap, and daytime sleepiness may signal a problem with nighttime sleep duration or quality (Sheldon, 2014).

Some sleep problems—-parasomnias, enuresis, SDB—may continue from the preschool period 
into the early school-aged period. School-aged children may increasingly have problems with getting the recommended sleep duration because wake time during the week is dictated by school start time. Children in this age group are also becoming more involved with electronic devices and consumption of caffeine during the day, which have been show to disrupt sleep patterns and next day function (Cain \& Gradisar, 2010; Lamontagne, Singh, \& Palosky, 2010). Parents, however, may not be as aware of sleep problems in school-aged children, because children at this age are more likely to put themselves to bed in the evening, and fall back to sleep themselves if they wake in the night.

\section{Adolescents}

As children move into puberty, considerable changes in sleep patterns take place. In natural conditions (no set bedtime or waketime), the amount of slow wave sleep decreases significantly from preto post-puberty. Pubertal children also experience a delayed sleep phase, naturally falling asleep later and waking later than pre-pubertal children. Based on study of natural and recovery sleep after sleep deprivation, Jenni et al. (2005) propose that homeostatic sleep pressure during the day accumulates more slowly in post-pubertal children, but that the waning of Process S remains comparable to pre-pubertal and pubertal children. Research has also found that total sleep time and REM sleep time needed remain constant across the pubertal process.

The primary sleep problem encountered in adolescence is daytime sleepiness, and cumulative sleep debt because of nighttime sleep restriction. As adolescents progress through puberty, daytime sleepiness increases, even in the absence of nighttime sleep deprivation. Post-pubertal adolescents tend to be sleepiness during the early to mid-afternoon (Carskadon et al., 1979; Crowley, Tarokh, \& Carskadon, 2014). Daytime sleepiness is compounded by the effects of sleep debt commonly seen in contemporary adolescents. As adolescents begin to exercise more independence, become more involved in social activities and sports, and receive more homework, bedtimes are often pushed later in the night. However, they must then wake up in time for early school start times. Reduced total sleep time in combination with natural daytime sleepiness can put adolescents at risk for serious attentional impairment. In addition to the 
detrimental effects this attentional impairment may have on academic achievement, it can have deadly consequences for young adults who are learning to drive.

\subsubsection{Measurement of sleep patterns}

Sleep patterns and the EEG structure of sleep may be studied with polysomnography (PSG), actigraphy (ACT), validated sleep instruments, or sleep diaries. The in-laboratory PSG is considered the "gold standard” for the evaluation of sleep according to the American Association of Sleep Medicine (Kushida, Littner, \& Morgenthaler, 2005). Before an in-laboratory PSG, a detailed history and physical examination is performed to evaluate normal sleep patterns and symptoms of sleep disorders. A PSG is performed by a trained polysomnographic technologist, and usually includes central EEG, respiratory effort, nasal and oral airflow, a microphone to record snoring, pulse oximetry, electrocardiogram, and video recording to document body positions during sleep. This recording can determine the total time in each sleep stage, total sleep time, sleep efficiency, sleep onset latency (time to fall asleep), REM sleep latency, wake after sleep onset, and arousals (Krishna, 2014; Roland et al., 2011). A disadvantage of PSG is that sleep quality is often poorer in the sleep lab with its associated equipment than at home. For example, the "first-night effect” occurs when sleep quality is poorer on the first night of a two night study in the PSG lab (Buckley et al., 2013).

Actigraphy uses a device about the size of a wristwatch to quantify sleep-wake patterns by measuring movement. The actigraph is worn on the wrist or ankle for several days and nights as individuals go about their regular activities. The actigraph registers frequency and amplitude of movements in 30- or 60-second periods. One benefit of ACT is that it's objectively measured activity patterns may estimate habitual sleep patterns more effectively than PSG because of being worn by an individual in their natural environment, avoiding the first-night phenomenon. ACT can be used to measure sleep latency, total sleep time, wake time after initial sleep onset, sleep efficiency (total sleep time/total time in bed) by recording episodes of activity and inactivity (Ancoli-Israel et al., 2003). 
Sleep questionnaires have been developed to specifically evaluate overall sleep, sleep initiation and maintenance, daytime sleepiness, sleep habits and sleep hygiene, beliefs about sleep, and multiple sleep dimensions (Lewandowski, Toliver-Sokol, \& Palermo, 2011). The most commonly used sleep questionnaire in the pediatric population is the Children's Sleep Habits Questionnaire (CSHQ), a 35-item, well-validated and commonly used parent-completed multidimensional instrument that assesses bedtime routine, sleep behavior, waking during the night and in the morning, and daytime sleepiness. Each item is scored on a 1 to 3 scale indicating how frequently a behavior occurs, with 1 corresponding to usually (5-7 days/week), 2 to sometimes (2-4 times/week), and 3 to rarely (0-1 time/week). Thus, a high score indicates more sleep problems than a low score. The CSHQ addresses sleep latency as how often the child falls asleep within 20 minutes of going to bed (Owens, Spirito, \& McGuinn, 2000).

Sleep diaries record information about time to bed, sleep onset latency, and number and length of night wakings, final awakening time in the morning, and subjective evaluation of sleep quality. Sleep diaries may take the form of simply writing information in a journal, shading in a bar graph with sleep time, or the standardized Consensus Sleep Diary (CSD). The CSD gathers the above information, and may also ask if final awakening time was earlier than planned, excess daytime sleepiness and napping during the day, caffeinate consumption during the day, and sleep medications taken (Carney et al., 2012; Luyster et al., 2015). It is recommended that sleep diaries capture at least five weekday nights to most accurately record adolescent bedtimes, sleep onset latency, and sleep duration (Short, Arora, Gradisar, Taheri, \& Carskadon, 2017). Sleep diaries may underestimate length of night awakenings, and overestimate total sleep time when compared with ACT recordings, although ACT may record movements during sleep as activity and quiet time while awake as sleep (Short, Gradisar, Lack, Wright, \& Carskadon, 2012).

\subsubsection{Support for investigation of sleep patterns in autism spectrum disorder}

Abnormal sleep patterns in ASD have long been recognized by researchers (Ornitz et al., 1969; Tanguay, Ornitz, Forsythe, \& Ritvo, 1976). Polysomnography studies have shown that, compared with typically 
developing [TD] peers, children with ASD have significantly lower average time in bed (mean: 9.2 $\pm 0.56 \mathrm{hrs}$ vs. $8.4 \pm 0.45 \mathrm{hrs}$ ), sleep period time (sleep onset to sleep end, mean: $8.9 \pm 0.45 \mathrm{hrs}$ vs. 7.7 $\pm 0.75 \mathrm{hrs}$ ), and total sleep time (sleep period time - wake after sleep onset + movements, mean: 8.7 \pm 0.40 hrs vs. $7.5 \pm 0.83 \mathrm{hrs}$, all $p \leq 0.02$; Elia et al., 2000). Krakowiak, Goodlin-Jones, Hertz-Picciotto, Croen, \& Hansen (2008) developed and performed factor analysis on the CHARGE Sleep History, their parent-report, 30-item sleep questionnaire for children with ASD similar to the well-validated Children's Sleep Habits Questionnaire (Owens et al., 2000), revealed two factors: sleep onset problems (7 items, $\mathrm{a}=0.79$ ) and night waking ( 4 items, $\mathrm{a}=0.81$ ). The most common sleep onset problem was trouble falling asleep ( $p<0.0001$ ), and the most common night waking problem was parasomnias, particularly waking up screaming during the night ( $p=0.0014)$.

Multiple ASD studies have confirmed that the most common complaints about sleep patterns in ASD are problems settling to bed, also referred to as Behavioral Insomnia of Childhood (Goldman et al., 2010; Hodge, Carollo, Lewin, Hoffman, \& Sweeney, 2014; Mazurek \& Petroski, 2015), trouble falling asleep (Aldinger, Lane, Veenstra-VanderWeele, \& Levitt, 2015; Allik et al., 2008; Fadini et al., 2015; Suzanne E Goldman et al., 2010; Krakowiak et al., 2008; Miano et al., 2007), frequent and/or long night wakings (Giannotti et al., 2008; Goldman et al., 2010; Hodge, Carollo, Lewin, Hoffman, \& Sweeney, 2014), early morning waking (Cotton \& Richdale, 2006; Gail Williams et al., 2004; Wiggs \& Stores, 2004), decreased sleep duration (Aldinger et al., 2015; Elrod \& Hood, 2015; Giannotti et al., 2011; Goldman et al., 2010) and daytime sleepiness (Giannotti et al., 2011; Goldman et al., 2010; May, Cornish, Conduit, Rajaratnam, \& Rinehart, 2015).

\subsubsection{Comparison of sleep in children with ASD and TD children}

A variety of objective and subjective measures reveal that bedtime difficulties—bedtime resistance, sleep anxiety, difficulty falling asleep-are significantly more common in children with ASD than their TD counterparts (Table 10). Significantly higher (worse) bedtime resistance, sleep anxiety, and sleep onset delay subscale scores on the CSHQ have been reported by numerous research groups in ASD samples 
when compared to TD samples (Flavia Giannotti et al., 2008, 2011; Hodge et al., 2014; May et al., 2015; Souders et al., 2009). Children in these samples range from 2.5- to 17-years-old, demonstrating the presence of sleep problems throughout childhood and adolescence. Giannotti et al. (2008) divided their ASD sample of children 2.5- to 8-years-old into developmentally regressed ( $n=70$; lost developmental skills after period of typical or mildly delayed development) and non-regressed children ( $\mathrm{n}=34$, symptoms apparent early in life with delayed developmental milestones; TD n=162; Williams, Brignell, Prior, Bartak, \& Roberts, 2015). They found that regressed children had significantly greater scores on the CSHQ bedtime resistance and sleep onset delay subscales than non-regressed children with ASD and TD children $(p<0.01)$. Regressed children also were found on parent-report sleep diaries to have significantly longer sleep onset latency than non-regressed children with ASD and TD children $(p<0.01)$. Using objective measures of sleep onset latency, several research groups have reported greater sleep onset latency in children with ASD than TD children aged 2- to 19-years, again demonstrating persistence of sleep problems through childhood and adolescence (ACT: Goldman et al., 2017; Lambert et al., 2016; Souders et al., 2009; PSG: Elrod \& Hood, 2015; Giannotti et al., 2011; Lambert et al., 2016). In a metaanalysis of ten studies, Elrod and Hood (2015) found a significant pooled mean delay in sleep onset latency of 10.9 minutes (95\% CI: 6.7 to 15.0 ) in children with ASD compared to TD children.

Children with ASD are also reported to have significantly more nighttime sleep problems than their TD peers. Using the CSHQ, children with ASD are reported to have significantly worse scores on night waking, parasomnia, and sleep duration subscale scores than TD children 2.5- to 17-years-old (Flavia Giannotti et al., 2008, 2011; Hodge et al., 2014; May et al., 2015; Souders et al., 2009). A common complaint of parents who have children with ASD, waking after sleep onset (WASO) was confirmed to be a significantly greater problem in children with ASD than TD children. Polysomnography revealed a greater percentage of total sleep time spent awake after sleep onset in children with ASD 5- to 10-years-old, and worse in regressed than non-regressed ASD $(p<0.05$; Giannotti et al., 2011); and ACT showed a greater number and length of WASO in children with ASD aged 4- to 15.5-years-old ( $p<0.05$; Allik, Larsson, \& Smedje, 2008; Souders et al., 2009). Sleep 
efficiency (total sleep time/time in bed) is reported to be significantly lower in children with ASD using ACT in children greater than 6-years-old ( $p<0.05$; Fletcher et al., 2016; Goldman et al., 2017); and using PSG in children 5- to 10-years old ( $p<0.05$; Giannotti et al., 2011). Overall, total sleep time has been shown to be significantly less in children with ASD than TD children throughout childhood and adolescence using parent-report sleep diaries (Flavia Giannotti et al., 2011; Hodge et al., 2014), CSHQ (Flavia Giannotti et al., 2008, 2011; Hodge et al., 2014; Souders et al., 2009), ACT (Goodlin-Jones, Tang, et al., 2008), and PSG (all $p<0.05$; Buckley et al., 2010; Giannotti et al., 2008, 2011; Miano et al., 2007)

Nighttime sleep problems are often followed by daytime sleepiness in all children, although less is published comparing ASD to TD. Children with ASD of all ages have received worse daytime sleepiness scores on the CSHQ daytime sleepiness subscale (2.5-12-years-old; $p<0.05$; Giannotti et al., 2008; May et al., 2015) and on the Sleep Disturbance Scale (3.7-19-years-old; $p<0.05$; Miano et al., 2007). Souders et al. (2009), however, did not find a significant difference between children with ASD and TD on the CSHQ daytime sleepiness subscale.

The notable shifts in sleep patterns that occur around puberty in TD children are believed to occur in adolescents with ASD as well. Allik et al.(2008) observed over the course of 2-3 years that a sample of adolescents with ASD (mean age time 1=11.1 years, time 2=13.7 years) developed significantly later sleep onset time (difference $=40.2 \pm 57.3$ minutes, $p=0.01$ ) and wake time (difference $=38.8 \pm 69.2$ minutes, $p<0.01)$. The same pattern was observed in age- and gender-matched TD controls. The amount of sleep needed appears to remain constant across pubertal stages (Carskadon et al., 1979). The average age of puberty (Tanner stages 2-4) in the United States is between 8-13-years for girls, and 9-14-years for boys; mean age of menarche is 12.2-12.8-years (Al-Sahab, Ardern, Hamadeh, \& Tamim, 2012; Cabrera, Bright, Frane, Blethen, \& Lee, 2014). Pubertal development was not well characterized in the SSC. For these reasons, children in SSC sample will be divided into three age groups: early childhood (4-8-years), middle childhood (9-13- years), and late childhood (14-18-years). 


\subsubsection{Intellect, ASD severity, and sleep}

It has been suggested that intellectual disability (ID; Full scale IQ $<70$ with functional deficits; World Health Organisation, 1996) may alter sleep patterns in children with ASD. Two recent studies have noted that ASD with ID was associated with sleep anxiety in children 2- to 5-years-old $(p<0.001)$, bedtime resistance and night wakings in children 6- to 18-years-old ( $p<0.05$; Mazurek \& Petroski, 2015), and short sleep duration in children 4- to 18-years-old ( $p<0.001$; Veatch et al., 2017). A meta-analysis of ten studies revealed that children with ASD and comorbid ID had significantly lower total sleep time than TD children (pooled difference of means $=-45$ minutes; 95\% CI: -62.6 to $-28.6, p<0.001$ ), while there was no significant difference in total sleep time between children with ASD and no ID compared to TD children $(p=0.316)$.

Severity of ASD may also contribute to abnormal sleep patterns in children. In a population of 1,224 children, those with parent-reported sleep problems (falling asleep and staying asleep) were observed to have higher ASD severity scores on the Autism Diagnostic Inventory-Revised social interaction, communication, and restricted, repetitive behavior subscales (Lord, Rutter, \& Le Couteur, 1994); and on the Vineland Adaptive Behavior Scale (Sparrow, Balla, Cicchetti, Harrison, \& Doll, 1984) communication, socialization, daily living skills, and motor skills subscales than children without sleep problems (Zachor \& Ben-Itzchak, 2016; $p<0.02$ ). Using the Autism Diagnostic Observation Scale (ADOS; Hus, Gotham, \& Lord, 2014; Lord et al., 1989) as an indicator of ASD severity, Veatch et al. (2017) observed that short sleep duration was significantly negatively correlated with ADOS social affect severity scores $(\rho=-0.06, p<0.02)$, though not with total ADOS severity score.

The correlations between sleep patterns and behavior are striking in children with ASD. Using the Pediatric Behavior Scale and the Checklist for Autism Spectrum Disorder, sleep problems—difficulty falling asleep and staying asleep, restlessness, parasomnias, waking too early, and less total sleep timewere significantly related to oppositional behavior, aggression, explosiveness, attention deficit and hyperactivity, anxiety, depression, and mood variability $(r=0.26-0.42, p<0.01$; Mayes \& Calhoun, 2009). By contrast, greater total sleep time significantly predicted better adaptive behavior, motor skills, 
socialization, and daily living skills on the Scales of Independent Behavior-Revised and the Vineland Adaptive Behavior Scale. It was even suggested that more hours of sleep per night may translate into higher full-scale IQ scores on age-appropriate Stanford-Binet or Wechsler Intelligence scales (Taylor, Schreck, \& Mulick, 2012). Some uncertainty exists, however, about whether behavior problems predict sleep patterns or the other way around.

\subsubsection{Medical conditions and sleep}

Two medical comorbidities that have been associated with sleep problems in children with ASD are gastrointestinal disturbance (GID: diarrhea, constipation, vomiting or reflux, abdominal pain, bloating, etc.) and seizures. Presence of epilepsy and epileptiform EEG abnormalities have both been reported as predictors of sleep problems (Giannotti et al., 2008; $p<0.01$ ). Aldinger, Lane, Veenstra-VanderWeele, and Levitt (2015) report that, among children with ASD and sleep problems in the SSC, the odds of having GID (23\% of sample) were almost two times higher than for children without sleep problems (OR=1.8, 95\% CI: 1.5-2.3), and the odds of having both GID and seizures (2.6\% of sample) was twice as high as children without sleep problems (OR=2.0, 95\% CI: 1.3-3.1). In the Autism Genetic Resource Exchange, Aldinger et al. also report that children with sleep problems, seizures, and GID had a two standard deviation lower Vineland Adaptive Behavior Scale composite score than children with none of the three comorbidities.

\subsubsection{Support for investigation of circadian genes and sleep patterns}

Components of sleep, such as onset, duration, and sleep stages, appear to be highly heritable based on monozygotic and dizygotic twin studies (Ambrosius et al., 2008; De Gennaro et al., 2008). Studies of twins have also revealed that diurnal preference-morning or evening-is about 50\% heritable (Koskenvuo, Hublin, Partinen, Heikkila, \& Kaprio, 2007), and habitual bedtime is about 22-25\% heritable (Gottlieb, O’Connor, \& Wilk, 2007). Multiple gene/hormone pathways expressed throughout the brain may contribute to regulation of sleep patterns. These include serotonin (Frank, Stryker, \& Tecott, 2002; 
Popa, El Yacoubi, Vaugeois, Hamon, \& Adrien, 2006), dopamine (Goel, Banks, Lin, Mignot, \& Dinges, 2011; Kume, Kume, Park, Hirsh, \& Jackson, 2005), histamine (Anaclet et al., 2009), adenosine (Bjorness, Kelly, Gao, Poffenberger, \& Greene, 2009; Stenberg et al., 2003), y-aminobutyric acid (GABA; WinskySommerer, 2009); cytokines (Clinton, Davis, Zielinski, Jewett, \& Krueger, 2011; Darko et al., 1995); orexin (Anaclet et al., 2009; Mochizuki et al., 2004); and melatonin/SCN (Isobe \& Nishino, 2004; Moore, Speh, \& Leak, 2002; Pandi-Perumal, Srinivasan, Spence, \& Cardinali, 2007). Some pathways, such as orexin (Anaclet et al., 2009; Ebrahim, Howard, \& Kopelman, 2002), dopamine (Andretic, van Swinderen, \& Greenspan, 2005; Kume et al., 2005), and histamine (Parks et al., 2014; Thakkar, 2011) provide arousal cues; while others, such as GABA (Alam et al., 2005; Goutagny, Luppi, Salvert, Gervasoni, \& Fort, 2005), adenosine (Basheer, Porkka-Heiskanen, Strecker, Thakkar, \& McCarley, 2000; Porkka-Heiskanen et al., 1997), and the cytokines, interleukin-1 $\beta$ and tumor necrosis factor promote sleep (Krueger, Rector, \& Churchill, 2007).

The most thoroughly studied and well-replicated pathway regulating timing, onset, and maintenance of sleep are the melatonin/SCN pathways (Table 1; Dardente \& Cermakian, 2007; Ko \& Takahashi, 2006). The SCN, the circadian pacemaker of the body, is a small group of compact neurons sitting atop the optic chiasma in the hypothalamus. The SCN is responsible for controlling the sleep-wake cycle, and coordinating physiologic functions according to circadian phase. When signaled by decreasing light through the retinohypothalamic pathway, the SCN stimulates the pineal gland to produce melatonin (Moore, 1996; Moore et al., 2002). The melatonin synthesis pathway is a four-step process carried out in the pineal gland via the expression of TPH2 (Carkaci-Salli et al., 2006; Zhang, Beaulieu, Sotnikova, Gainetdinov, \& Caron, 2004), DDC (Lauritsen et al., 2002; National Center for Biotechnology Information, 2014b; Sumi-Ichinose, Ichinose, Takahashi, Hori, \& Nagatsu, 1992), AANAT (Blomeke, Golka, Griefahn, \& Roemer, 2008; Coon et al., 1996), and ASMT (Huafang, Donohue, Klein, \& Mcbride, 1992; National Center for Biotechnology Information, 2014b; L. Wang et al., 2013). When melatonin binds to the SCN's MTNR1A and MTNR1B receptors, it entrains the circadian rhythm to 24-hours. In general, MTNR1A suppresses SCN neuronal excitability at night, whereas MTNR1B receptors shift phase 
by switching the SCN from daytime high-electrical, low hormone mode to nighttime low-electrical, high hormone mode (Dubocovich, Benloucif, \& Masana, 1996; Liu et al., 1997).

Oscillations in the expression of core SCN genes influence sleep by driving phase shifts in two main autoregulatory feedback loops. At the center of both loops are the core transcriptional regulators CLOCK and ARNTL. In the first loop, CLOCK and ARNTL heterodimerize to activate the rhythmic transcription of PERIOD (PER1, PER2, PER3) and CRYPTOCHROME (CRY1, CRY2) genes (King et al., 1997; Kondratov et al., 2003; National Center for Biotechnology Information, 2014a, 2014a). DBP, although not necessary for maintenance of circadian rhythm, enhances CLOCK:ARNTL activation of PER (Lopez-Molina, Conquet, Dubois-Dauphin, \& Schibler, 1997). Activated by the CLOCK:ARNTL heterodimer, $P E R$ and $C R Y$ proteins accumulate in the cytoplasm throughout the day and then form PER:CRY heterodimers that enter the nucleus and repress the CLOCK:ARNTL complex (Nieto et al., 2015; UniProtKB, 2014a, 2014b). This repression may be aided by TIMELESS (Sangoram et al., 1998). Degradation of PER and $C R Y$ by casein kinase (CSNK1D, CSNK1E) phosphorylation allows the feedback loop to begin again (National Center for Biotechnology Information, 2014).

The second loop regulates expression of $A R N T L$ and activates genes that carry messages within and out of the SCN. The nuclear orphan receptor genes, RORA/B upregulate, and NR1D2 downregulates, expression of ARNTL. BHLHE40/41 (Kawamoto et al., 2004; Nakashima et al., 2008; Sato et al., 2004) and CIART (Goriki et al., 2014) interfere with the activation of core circadian genes by ARNTL. The SCN distributes its circadian message to receptors in the brain and peripheral tissues using several neurotransmitters (Born, Kellner, Uthgenannt, Kern, \& Fehm, 1992; Scheer, Ter Horst, Van der Vliet, \& Buijs, 2001; Vázquez-Palacios, Retana-Márquez, Bonilla-Jaime, \& Velázquez-Moctezuma, 2001) or autonomic nerves to deliver electrical messages directly to targeted tissues (Bamshad, Song, \& Bartness, 1999; Buijs et al., 1999; Buijs, Chun, Niijima, Romijn, \& Nagai, 2001; Gerendai, Toth, Boldogkoi, Medveczky, \& Halasz, 2000; Kalsbeek, Fliers, Franke, Wortel, \& Buijs, 2000). PROK2 acts as a key signaling molecule within the SCN and to other regions of the brain (Cheng et al., 2002; Masumoto et al., 2006). The primary neurotransmitter carrying messages from the SCN to the pineal gland is GABA, 
encoded by the GAD1 and GAD2 genes (Erlander, Tillakaratne, Feldblum, Patel, \& Tobin, 1991; Liu \& Reppert, 2000; O’Hara, Andretic, Heller, Carter, \& Kilduff, 1995).

Table 1: Melatonin and SCN Genes

\begin{tabular}{|c|c|c|c|}
\hline Gene & Name & Function & Associations \\
\hline ТPH2* & tryptophan hydroxylase 2 & $\begin{array}{l}\text { 5-hydroxylation of L-tryptophan to } \\
\text { 5HTP }\end{array}$ & \\
\hline$D D C^{*}$ & dopa decarboxylase & Decarboxylation of 5HTP to $5 \mathrm{HT}$ & \\
\hline AANAT & $\begin{array}{l}\text { aralkylamine } \mathrm{N} \\
\text { acetyltransferase }\end{array}$ & $\begin{array}{l}\mathrm{N} \text {-acetylation of } 5 \mathrm{HT} \text { to } \mathrm{N} \\
\text { acetylserotonin }\end{array}$ & $\begin{array}{l}\text { DSPS (Hohjoh et al., 2003), } \\
\text { short sleep duration (Wang, } \\
\text { Lee, \& Lee, 2004) }\end{array}$ \\
\hline$A S M T^{*}$ & $\begin{array}{l}\text { acetylserotonin } \mathrm{O} \\
\text { methyltransferase }\end{array}$ & $\begin{array}{l}\text { O-methylation of } \mathrm{N} \text {-acetylserotonin to } \\
\text { melatonin }\end{array}$ & $\begin{array}{l}\text { Sleep efficiency in ASD } \\
\text { (Melke et al., 2008) }\end{array}$ \\
\hline MTNR1A* & melatonin receptor $1 \mathrm{~A}$ & Suppresses SCN neuronal firing & \\
\hline MTNR1B* & melatonin receptor $1 \mathrm{~B}$ & Induces circadian phase shifting & $\begin{array}{l}\text { Insomnia in ASD (Yang et } \\
\text { al., 2016) }\end{array}$ \\
\hline CLOCK* & clock circadian regulator & $\begin{array}{l}\text { Transcription factor, with } A R N T L \\
\text { activates transcription of } P E R, C R Y \text {, } \\
\text { NR1D2, RORA/B, PROK2, } \\
\text { TIMELESS }\end{array}$ & $\begin{array}{l}\text { Narcolepsy (Moreira et al., } \\
\text { 2005), sleep duration } \\
\text { (Allebrandt et al., 2010) }\end{array}$ \\
\hline$A R N T L$ & $\begin{array}{l}\text { aryl hydrocarbon receptor } \\
\text { nuclear translocator-like }\end{array}$ & $\begin{array}{l}\text { Transcription factor, with } C L O C K \\
\text { activates transcription of } P E R, C R Y \text {, } \\
\text { NR1D2, RORA/B, PROK2, } \\
\text { TIMELESS }\end{array}$ & $\begin{array}{l}\text { Altered sleep patterns, later } \\
\text { sleep onset and wake time in } \\
\text { elderly (Evans et al., 2013) }\end{array}$ \\
\hline PER1* & period circadian clock 1 & $\begin{array}{l}\text { Feedback regulation of } \\
C L O C K: A R N T L\end{array}$ & $\begin{array}{l}\text { Extreme diurnal preference } \\
\text { (Carpen, von Schantz, Smits, } \\
\text { Skene, \& Archer, 2006) }\end{array}$ \\
\hline PER2 & period circadian clock 2 & Maintains clock gene RNA rhythms & $\begin{array}{l}\text { FASPS (Shanware et al., } \\
\text { 2011), DSPS (Hamet \& } \\
\text { Tremblay, 2006) }\end{array}$ \\
\hline PER3* & period circadian clock 3 & Maintains cycle length & $\begin{array}{l}\text { DSPS (Archer et al., 2010), } \\
\text { extreme diurnal } \\
\text { preference (Archer et al., } \\
\text { 2003) }\end{array}$ \\
\hline CRY1 & cryptochrome 1 & $\begin{array}{l}\text { Repression of CLOCK:ARNTL; } \\
\text { translocation of PER to nucleus }\end{array}$ & \\
\hline CSNK1D* & casein kinase 1 , delta & $\begin{array}{l}\text { Phosphorylates } P E R \text { induces } \\
\text { translocation of } P E R 3 \text { to nucleus }\end{array}$ & FASPS (Brennan et al., 2013) \\
\hline CSNK1E & casein kinase 1 , epsilon & $\begin{array}{l}\text { Ubiquinates, translocates } P E R 3 \text { to } \\
\text { nucleus; phosphorylation of } P E R\end{array}$ & $\begin{array}{l}\text { DSPS, non-24h sleep-wake } \\
\text { syndrome (Takano et al., } \\
\text { 2004) }\end{array}$ \\
\hline
\end{tabular}




\begin{tabular}{|c|c|c|c|}
\hline RORA* & $\begin{array}{l}\text { RAR-related orphan receptor } \\
\text { A }\end{array}$ & $\begin{array}{l}\text { Activates } C L O C K \text { and } A R N T L \\
\text { expression }\end{array}$ & $\begin{array}{l}\text { Rigid circadian type (Etain et } \\
\text { al., 2014), early } \\
\text { morning awakening in } \\
\text { depression (Utge et al., 2010) }\end{array}$ \\
\hline RORB & $\begin{array}{l}\text { RAR-related orphan receptor } \\
\text { B }\end{array}$ & Activates ARNTL expression & $\begin{array}{l}\text { Free-running rhythmicity } \\
\text { (André et al., 1998) }\end{array}$ \\
\hline NR1D2 & $\begin{array}{l}\text { Nuclear receptor subfamily } \\
1 \text { group D member } 2\end{array}$ & $\begin{array}{l}\text { Transcription factor, represses ARNTL } \\
\text { expression }\end{array}$ & \\
\hline BHLHE40 & $\begin{array}{l}\text { Basic HLH family member } \\
\text { E40 }\end{array}$ & $\begin{array}{l}\text { Represses CLOCK:ARNTL activation } \\
\text { of PER1 }\end{array}$ & $\begin{array}{l}\text { Non-24 hour sleep-wake } \\
\text { syndrome (Kripke et al., } \\
\text { 2014) }\end{array}$ \\
\hline BHLHE41 & $\begin{array}{l}\text { Basic HLH family member } \\
\text { E41 }\end{array}$ & $\begin{array}{l}\text { Represses CLOCK:ARNTL activation } \\
\text { of PER1 }\end{array}$ & $\begin{array}{l}\text { Short sleep duration (He et } \\
\text { al., 2009) }\end{array}$ \\
\hline CIART & $\begin{array}{l}\text { Circadian associated } \\
\text { repressor of transcription }\end{array}$ & $\begin{array}{l}\text { Interferes with } A R N T L \text { to repress } \\
C L O C K: A R N T L \text { activity }\end{array}$ & \\
\hline$G A D 1 * / 2$ & Glutamate decarboxylase & $\begin{array}{l}\text { Catalyze production of GABA, the } \\
\text { main SCN output neurotransmitter }\end{array}$ & \\
\hline PROK2 & Prokineticin 2 & Transmits circadian signals from SCN & $\begin{array}{l}\text { Sleep duration (Gottlieb et } \\
\text { al., 2007), reduced } \\
\text { rhythmicity (J. Li et al., } \\
\text { 2006) }\end{array}$ \\
\hline$D B P$ & $\begin{array}{l}\text { D Site of Albumin Promoter } \\
\text { (Albumin D-Box) Binding } \\
\text { Protein }\end{array}$ & $\begin{array}{l}\text { Enhances activation of } P E R \text { regulates } \\
\text { clock output signals }\end{array}$ & $\begin{array}{l}\text { Sleep consolidation (Franken, } \\
\text { Lopez-Molina, Marcacci, } \\
\text { Schibler, \& Tafti, 2000), } \\
\text { sleep duration (Yamajuku et } \\
\text { al., 2011) }\end{array}$ \\
\hline TIMELESS* & Timeless Circadian Clock & $\begin{array}{l}\text { Interacts with } P E R \text { to repress } \\
\text { CLOCK:ARNTL }\end{array}$ & $\begin{array}{l}\text { Sleep disturbance in } \\
\text { depression (Utge et al., 2010) } \\
\text { and ASD (Yang et al., 2016), } \\
\text { later sleep onset (Etain et al., } \\
\text { 2014) }\end{array}$ \\
\hline
\end{tabular}

Variations in core melatonin and SCN genes have been correlated with abnormal sleep patterns in individuals with ASD. Spurred by reports of abnormal melatonin levels in individuals with ASD, (Kulman et al., 2000; Leu et al., 2011; Nir, Meir, Zilber, Knobler, \& Hadjez, 1995; Tordjman, Anderson, Pichard, Charbuy, \& Touitou, 2005) Melke et al.(2008) examined variations in ASMT in 250 individuals with ASD, and 255 controls. Two promoter polymorphisms were found significantly more frequently in individuals with ASD (rs4446909 and rs5989681, $p<0.000$ ) leading to a substantial decrease in ASMT activity $\left(p=2 \times 10^{-12}\right)$ and melatonin level $\left(p=3 \times 10^{-11}\right)$ in two triads (mother, father, child). In addition, a splice-site mutation (IVS5+2T>C) was correlated in ASD with decreased sleep efficiency (70-82\% vs. 
$>85 \%$ in controls) and increased night wakings (17-22 times/hr vs. $<10$ times/hr in controls) in one triad. More recently, Veatch et al. (2015) confirmed a high frequency of two ASMT polymorphisms previously reported by Melke et al. (rs4446909 and rs5989681) in a group of fifteen unrelated children with ASD and sleep onset delay. Yang et al.(2016) took a broader approach, examining 18 melatonin and SCN genes in individuals with ASD with $(\mathrm{n}=14)$ and without self-reported abnormal sleep patterns $(\mathrm{n}=14)$ and controls ( $\mathrm{n}=23)$. Six missense changes found exclusively in individuals with ASD and abnormal sleep problems were observed in CLOCK (c.2551A>G), MTNR1B (с.974G>A), PER3 (с.1447C>T, c.1361G>A), NIR1D1 (c.58A>C), and TIMELESS (c.1493T>C). These studies are limited in their ability to identify meaningful associations between genomic and biochemical variants and sleep patterns by their small sample sizes and choice of only one gene.

Intriguingly, and in support of an abnormality in melatonin synthesis or use by the body, a multitude of studies have shown that exogenous melatonin supplementation in children with ASD significantly improves sleep latency, duration of sleep, and sleep efficiency (Cortesi, Giannotti, Sebastiani, Panunzi, \& Valente, 2012; Gringras, Nir, Breddy, Frydman-Marom, \& Findling, 2017; Malow et al., 2012; Wirojanan et al., 2009; Wright et al., 2011). In a recent meta-synthesis of eight systematic reviews examining melatonin supplementation in children with ASD, it was concluded that there is strong evidence for a positive effect of melatonin on sleep latency, total sleep time, bedtime resistance, and need for co-sleeping; and moderate evidence for a positive effect on night wakings, activity during the night, and the longest sleep episode without waking (Cuomo et al., 2017). Rossignol and Frye (2011; included in Cuomo et al. meta-synthesis) showed in a meta-analysis of five randomized, double-blind, placebo-controlled studies of melatonin supplementation revealed significant improvements from baseline in total sleep time (73 min greater vs. 44 min with placebo) and sleep latency (66 min less vs. 39 min with placebo; both $p<0.01$ ). An open-label trial of controlled-release melatonin supplementation report sustained improvements in sleep latency, bedtime resistance, total sleep time, night wakings, and need for a parent to co-sleep up to 2-years after starting melatonin $(p<0.001)$, and a relapse in sleep problems when taken off the melatonin (NS; Giannotti, Cortesi, Cerquiglini, \& Bernabei, 
2006). These studies report that melatonin supplements were well-tolerated with mild to no adverse effects.

\subsection{PRELIMINARY STUDIES}

\subsubsection{Simons Simplex Collection Sleep Interview}

In order to study sleep patterns in the SSC sample, psychometric evaluation was performed on items pertaining to sleep from the SSC medical history form. The medical history form used 18 questions (“Does your child currently or has your child ever had...”) to investigate sleep habits—bedtime, waketime, sleep duration; daytime problems as a result of sleep patterns-difficulty waking, naps, daytime sleepiness; and associated symptoms—sleep disordered breathing, parasomnias, enuresis. Correlational analyses and exploratory factor analysis was executed on this data from the sample of 2,737 children with ASD aged 4-18 years to develop the Simons Simplex Collection Sleep Interview (SSCSI; Table 10). The resulting cross-sectional, parent-report questionnaire is comprised of ten dichotomous (yes/no) items in three subscales: Nighttime Problems (bedtime problems, need to lay down with child, difficulty falling asleep, frequent/prolonged awakenings), Daytime Problems (difficulty waking, excess daytime sleepiness, long/frequent naps, regular duration is not enough), and Sleep Duration Problems (irregular bedtime and/or waketime, no regular duration). An eleventh question asks for the child's regular sleep duration in hours. The SSCSI is scored by giving one point for each "yes" response to the ten dichotomous items, and also one point if the regular sleep duration is outside the duration recommended by the National Sleep Foundation for the child's age (Hirshkowitz et al., 2015). The points

for these eleven questions are summed to yield a composite score (range $=0-11$ ), and three subscale scores (Nighttime Problems and Daytime Problems: range = 0-4; Sleep Duration Problems: range = 0-2). 
These scores will be used as the dependent variables in the proposed dissertation project. (See Manuscript 1, Psychometric properties of the Simons Simplex Collection Sleep Interview, for a full description.)

\subsection{RESEARCH DESIGN AND METHODS}

This study was a secondary analysis of data from the SSC, a large and very well characterized GWAS of physical, behavioral, and cognitive correlates of ASD. Due to the nature of this sample, we were able to test for association using quantitative trait techniques to examine associations between child characteristics (age, sex, intellect, ASD severity, and medical conditions) and sleep patterns, and between gene variants and sleep patterns in children with ASD.

\subsubsection{Parent study design and sample}

The Simons Foundation Autism Research Initiative (SFARI) began in 2003 with the goal of improving the scientific understanding of ASD. One of their major projects was the SSC, a sample of 2,744 simplex families from 12 sites in the United States. (Simplex means only one subject with ASD, termed the proband, in a nuclear family; and, for the SSC, no other family member up to third degree relatives affected with ASD.) The goal of the SSC was to identify highly penetrant, rare de novo mutations and copy number variants associated with ASD. Its data have been used to study genomics of cognition and behavior in ASD (Frazier, Georgiades, Bishop, \& Hardan, 2014), intellectual disability (Gamsiz et al., 2013), the broad autism phenotype (Davidson et al., 2014), and the impact of copy number variants on ASD (Luo et al., 2012). More than thirty instruments were used, or interviews conducted, to collect many other variables with which we can study ASD in the future. These include demographics; family health,

psychiatric, developmental, and environmental exposure histories; proband medical, developmental, treatment, school, and ASD histories; and current proband comorbidities, intelligence, behavior, motor 
skills, and speech. Blood samples were taken from biological parents, the proband, and one full sibling, if available (Fischbach \& Lord, 2010; Simons Foundation Autism Research Initiative, 2014).

Families were recruited from clinics at the University of Michigan, Yale University, Emory University, Columbia University, Vanderbilt University, McGill University, Harvard University (Children's Hospital of Boston), the University of Washington, the University of Illinois (Chicago), the University of Missouri, the University of California-Los Angeles, and the Baylor College of Medicine. Local service providers and parent and advocacy groups were enlisted to aid in recruitment, in addition to web postings and radio and television ads.

Children were included if they were between 4- and 18-years-old; met the criteria for ASD based on the Autism Diagnostic Observation Schedule [ADOS], Autism Diagnostic Interview-Revised [ADIR], and Diagnostic and Statistical Manual of Mental Disorders-IV-Text Revised; and had a non-verbal mental age of at least 24-months (in probands 4- to 7-years-old), or 30-months (in probands $\geq 7$-yearsold). Probands were excluded if they were born at $<36$-weeks and had a birth weight $<2,000$ grams; were born with a significant injury, abnormality, or disorder affecting the brain; had extended prenatal or birth complications; had fragile $\mathrm{X}$ or Down syndrome; had sensory or motor disorders that would confound diagnostic measures; had a history of severe nutritional or psychological deficiency; or were not fluent in English (Simons Foundation Autism Research Initiative, 2014). Informed consent was obtained for the SSC study from parents or caregivers, and assent was obtained from children as required by local institutional review boards.

Probands were strictly phenotyped through parent interview, medical history, and multiple wellvalidated instruments. Children were given a clinical best estimate diagnosis of autism or ASD based on observation, medical history and chart review, and ADOS and ADI-R scores. Diagnoses were based on the Diagnostic and Statistical Manual of Mental Disorders-IV-Text Revised [DSM-IV-TR] (Simons Foundation Autism Research Initiative, 2014). 


\subsubsection{Study procedures}

Approval was obtained from the University of Pittsburgh Institutional Review Board for the current study. Data was de-identified by the SFARI prior to receipt by the present researchers. All phenotypic and genomic data were obtained from the SSC through the SFARI software platform, SFARI Base. Demographic data (age, sex, etc.) were collected when families entered the SSC study. The present study tried to capture normal changes in sleep patterns by dividing the sample into three age groups called early (4-8-years), middle (9-13- years), and late (14-18-years) childhood. As analysis of the sample (Johansson, Feeley, Dorman, \& Chasens, n.d.), revealed associations between sleep problems and presence or absence of GID, non-verbal IQ (NVIQ), and ADOS total score, these variables, with the addition of seizures, will be used as covariates in analysis. The medical condition, GID, was defined as parent reports of bloating/excess gas, celiac disease, constipation, diarrhea, ulcers, gastroesophageal reflux disease, inflammatory bowel disease (Crohn's disease, ulcerative colitis), irritable bowel syndrome, abdominal pain, unusual stools, vomiting, and/or other GID; and seizures as parent reports atonic drop, infantile spasms; or partial complex, grand mal, petit mal, simple partial, and/or other seizures. Presence or absence of GID and seizures was determined using data from the medical history interview conducted by the parent study at baseline. Participants were excluded from the present study if more than two SSCSI items were missing or coded unknown, or if the child was missing both ADOS and IQ measures.

\subsubsection{Genotype data collection}

Variants used in analysis of genes and sleep problems were identified using gene expression data from the CommonMind Consortium (CMC; Fromer et al., 2016), the Genotype-Tissue Expression project (GTEx; The GTEx Consortium, 2015), HaploReg v.4 (Ward \& Kellis, 2016), and previous literature (Melke et al., 2008; Veatch et al., 2015; Yang et al., 2016) (Table 12). The CMC used RNA Seq data from dorsolateral prefrontal (DLPFC) brain tissue of 279 control individuals and 258 adult individuals diagnosed with neuropsychiatric disorders. Using the RNA sequencing data and genotypes from the same subjects, gene 
expression and how it relates to genetic variation in candidate genes was assessed at almost 10 million loci using regression methods. This identified expression quantitative trait loci (eQTLs), SNPs or genomic variants that affect the expression of one or more genes (Albert \& Kruglyak, 2015).

Figure 3 displays the flow of variant selection from the CMC, GTEx, HaploReg, and variants previously identified by other groups as related to sleep in ASD. For the current study, gene expression was analyzed in cis- (within $1 \mathrm{Mb}$ of gene) variants with a false discovery rate threshold FDR $\leq 0.05$. This revealed that 17 of the 25 genes are expressed in substantial quantities in the CMC DLPFC data (TPH2, CLOCK, ARNTL, PER1/2/3, BHLHE40/41, DBP, GAD1/2, NR1D2, RORA/B, CSNK1D/E, CRY1), and identified 5,133 cis-eQTLs whose genotypes correlate with the total abundance of transcripts of its nearby gene. From this list, all eQTLs with a $p$-value $<0.001$ were chosen. Using genotypes from 1000 Genomes (Auton et al., 2015), the eQTLs were analyzed using the independent pairwise function in Plink, which prunes the list based on linkage disequilibrium (LD). The settings used were a window of 50 SNPs, moving by 5 SNPs at each step, and an $r^{2}=0.5$. When eQTL were not present in the SSC data, proxy eQTL in significant LD were substituted; SNAP Proxy Search was used to find proxy eQTLs (Broad Institute, 2015). This resulted in 72 eQTL; 49 were available in SSC data (32 proxy eQTL were substituted). eQTLs were not found in the CMC analysis for CRY1.

To identify eQTLs for 7 of the remaining 8 genes (MTNR1B, CRY1, DDC, AANAT, PROK2, CIART, TIMELESS), data was used from the GTEx project (The GTEx Consortium, 2015). The GTEx project aimed to further the understanding of functional genomic variation, including how this variation impacts human disease and traits, by identifying eQTLs for all genes in a variety of human tissue types. For the 7 genes, 1,051 eQTLs were available in the GTEx database; 556 of these were unique (not repeated). Using genotypes from 1000 Genomes, Plink pruned these down to 40 eQTLs. The settings used were a window of 50 SNPs, moving by 5 SNPs at each step, and an $r^{2}=0.5$. Of the 40 eQTLs, 16 were available in the SSC data (12 proxy eQTLs were substituted).

HaploReg acquired SNP and eQTL results from GTEx pilot analysis v.6 (The GTEx Consortium, 2015), the GEUVADIS project (Lappalainen et al., 2013)s, the GRASP database (Eicher et al., 2015), and 
a dozen studies (Fairfax et al., 2014; Gibbs et al., 2010; Grundberg et al., 2011; Hao et al., 2012; Koopmann et al., 2014; Q. Li et al., 2014; Montgomery et al., 2013; Ramasamy et al., 2014; Schadt et al., 2008; Stranger et al., 2009; Westra et al., 2013; Zou et al., 2012). For MTNR1A, 2,149 SNPs were found in the Haploreg v.4.1 program using the location of the gene plus 50KB on each side (chr4:187404809187526537). Of these, 12 variants had data for expression. The 12 eQTLs were analyzed using the independent pairwise function in Plink, using the previous settings. This resulted in 8 eQTL; 3 were available in SSC data (1 proxy eQTL was used).

From previous literature on sleep patterns in individuals with ASD, 26 variants were identified from 10 genes (ASMT, CLOCK, CSNK1D/E, MTNR1A/B, NR1D1, PER1/2/3, TIMELESS). Four of these variants were available in the SSC data (2 proxies were used): ASMT rs4446909 was identified by Melke et al. (2008) and Veatch et al. (2015); PER2 rs934945, PER3 rs10462020 (proxy: rs10462021), and PER3 rs228697 (proxy: rs228690) were identified by Yang et al. (2016).

In all, 72 independent eQTLs or SNPs were identified (Table 12: CMC = 50, GTEx = 16, Haploreg $=3$, literature $=4$ ). The variants from CMC, GTEx, and Haploreg are eQTLs or putative eQTLs; the four variants from literature are SNPs for which their effect on expression was not investigated. The 72 eQTLs and SNPs will henceforth be referred to as "variants.” When proxy eQTL were required, SNAP Proxy Search was used to find proxy eQTLs (Broad Institute, 2015).

Individuals in the SSC sample were genotyped using either Illumina 1Mv1 (approximately 325 families), Illumina 1Mv3 Duo (approximately 1,160 families), or Illumina HumanOMNI 2.5 (approximately 625 families) arrays. The same array version was used to analyze all members of a family. 


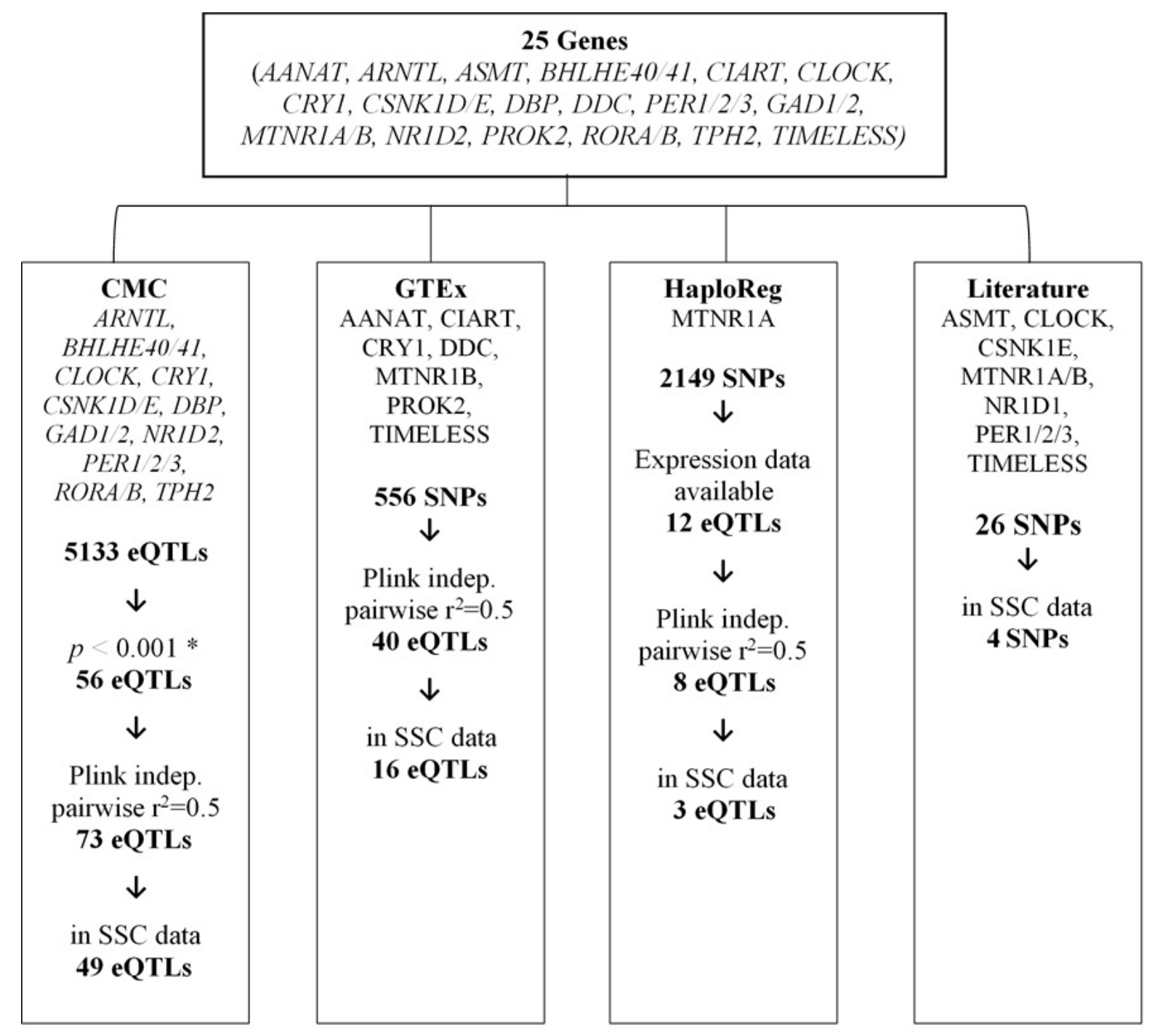

* Genotypes that significantly correlate with the total abundance of transcripts of its nearby gene

Figure 3: Flowchart of Genetic Variant Selection 


\subsubsection{Instruments}

\subsubsection{Sleep}

The SSCSI, an 11-item parent-report questionnaire, was used for assessing bedtime and nighttime sleep problems, daytime function, and sleep duration problems (Table 10). The instrument was developed from questions in the SSC medical history that were asked at a clinic visit or by telephone interview of parents within three months of study initiation. The SSCSI is comprised of three subscales: Nighttime Problems, Daytime Problems, and Sleep Duration Problems. The scoring rubric consists of individual items that are scored as 0 for "no" and 1 for "yes". A continuous composite score is made by summing the ten dichotomous items and adding one point for sleep duration that was outside the National Sleep Foundation recommendations for the child's age (Hirshkowitz et al., 2015). Composite scores range from 0 to 11 , with higher scores indicating worse sleep. Scores for the Nighttime Problems and Daytime Problems subscales range from 0 to 4, and scores for the Sleep Duration Problems subscale range from 0 to 2 (reviewed in Johansson, Feeley, Chasens, in press).

\subsubsection{IQ measures}

Intelligence is operationalized by the IQ measures as characteristics of cognitive abilities (verbal receptive and expressive abilities, nonverbal reasoning, and spatial abilities) and functional skills (processing speed, working memory, motor skills). Four norm-referenced instruments were used to quantify IQ in the SSC sample depending on chronologic and mental age (Table 11). Norm-referenced measures are those standardized by the developers of the measure on a group of individuals chosen to represent the general characteristics (ex. age, sex, ethnicity/race, socioeconomic status, geographical region) of those who will be measured with the instrument. Norm-referenced measures are mapped onto a scale so that scores are a rank that indicate the test-taker's performance relative to the normalization sample. Instruments are then standardized so that, although raw scores are comparable between instruments, standardized scores are on a common metric across instruments. This is done by mapping 
raw scores from the individual test onto a common scale with a total score mean $=100$ and standard deviation $=15$. Thus, each scale produces a common standardized verbal IQ (VIQ), nonverbal IQ (NVIQ), and full-scale IQ (FSIQ) score that can be used to quantify and compare IQ across instruments. The established cut-off for intellectual disability (ID), described by the Autism and Developmental Disabilities Monitoring Network as, a condition in which “a person has difficulty learning at an expected level and functioning in daily life” (Centers for Disease Control and Prevention, 2016), is an IQ test score $\leq 70$.

There are limitations to the standardization and measurement process for intellegence measures. Each measure uses different items to quantify IQ, although they are generally in common domains. IQ is often underestimated in individualas with ASD, frequently because of difficulty completing a standardized test (Bolte, Dziobek, \& Poustka, 2009). Discrepencies also often occur between nonverbal and verbal IQ scores in individuals with ASD. A primary reason for this is because of verbal and/or language deficits inherent in the disorder. Thus, tests that rely on language skills may not accurately reflect the child's actual intelligence and functional abilities (Joseph, Tager-Flusberg, \& Lord, 2002). For this reason, NVIQ scores were utilized in the present study, and scores were categorized into five groups. Nonverbal IQs above 70 were divided by the population mean (mean=100). Those below 70 were divided by population standard deviation $(S D=15)$ down to $N V I Q=40$. Based on recommendations from the American Association of Intellectual and Developmental Disabilities (Tasse, Luckasson, \& Nygren, 2013), NVIQ scores below 40 were condensed into one category.

The Differential Ability Scales-II (DAS-II) is a standardized battery of tests measuring a range of cognitive abilities in children with mental age 2 years 6 months through 17 -years-old. The DAS-II consists of 20 independent subtests within 3 item clusters measure verbal comprehension, nonverbal reasoning skills, and spatial abilities yielding norm-referenced ability scores, $t$-scores and confidence intervals, cluster scores, and percentile ranks. Six core subtests contribute to an overall General Conceptual Ability score.(Elliott, 2012; PsychCorp, 2016) The DAS-II has two versions used for younger children and school-aged children. The DAS-II Early Years is divided into a lower level for children with 
mental age 2 years 6 months to 3 years 5 months with 4 core subtests, and an upper level for children with mental age 3 years 6 months to 6 years 11 months, and has 6 core subtests. The DAS-II School Age is used for children with mental age 7 years to 17 years 11 months, and has 6 core subtests. Additional subtests are available for each of the three levels to measure things like school readiness, working memory, and processing speed. The SSC primarily used the DAS-II (85.0\%), with almost equal proportions using the Early Years (50.9\%) and School-Age (49.1\%) versions (Elliott, 2012; PsychCorp, 2016).

The Weschler Intelligence Scale for Children-Fourth Edition (WISC-IV) is a standardized intelligence test used in children with mental age 6 years to 16 years 11 months. The WISC-IV measures intellectual ability and cognitive processing with ten core and five supplemental subtests resulting in four index scores: verbal comprehension, perceptual reasoning, working memory, and processing speed. The scale has good reliability in standard samples (Full scale: $\alpha=0.88-0.97$, subtests: $\alpha=0.70-0.90$ ) and specialeducation samples (full-scale not calculated, subtests: $\alpha=0.82-0.93$; Williams, Weiss, \& Rolfhus, 2003). The scale has been used in ASD samples (Khor, Gray, Reid, \& Melvin, 2014; Oliveras-Rentas, Kenworthy, Roberson, Martin, \& Wallace, 2013; P. E. Williams et al., 2003).

The Wechsler Abbreviated Scale of Intelligence (WASI) is a standardized intelligence test used in individuals with mental age 6 years to 90 years 11 months. The WASI is a shortened version of the WISC-IV and Wechsler Adult Intelligence Scale, and has the same standardized scoring as the WISC-IV. The WASI has a 2-scale version resulting in VIQ, PIQ, and FSIQ; and a 4-scale version resulting in just FSIQ (Axelrod, 2002; Canivez, Konold, Collins, \& Wilson, 2009; Goldstein \& Mazefsky, 2013; McCrimmon \& Smith, 2012). The WASI has good reliability in a standard sample of children $(\alpha=0.81$ 0.97) and adults ( $\alpha=0.84-0.98$; The Psychological Corporation, 1999), and excellent reliability in a sample of adults with neurologic or psychiatric disorders ( $\alpha=0.96-0.98$; Axelrod, 2002).

The Mullen Scales of Early Learning (MSEL) is a standardized cognitive test used with children from birth to a mental age of 5 years 8 months. The MSEL produces an Early Learning Composite score which measures cognitive abilities with five subscales: Gross Motor, Visual Reception, Fine Motor, 
Receptive Language, and Expressive Language. The Gross Motor subscale is only used for children up to 33 months. Scores are reported as $T$ scores for subscales (mean=50, $\mathrm{SD}=10$ ) and standardized scores for the full scale (mean=100, SD=15; Bradley-Johnson, 1997). The MSEL has been used with TD children and those with ASD (Akshoomoff, 2006; Bishop, Guthrie, Coffing, \& Lord, 2011). The Early Learning Composite has good reliability ( $\alpha=0.83-0.95$; Dumont, Cruse, Alfonso, \& Levine, 2000).

\subsubsection{ASD severity measures}

ASD severity was measured with the ADOS, a semi-structured observational instrument used for diagnosis of ASD and classification of severity. The measures that make up the ADOS total score are operationalized as characteristics of social responsiveness, communication, language delay, and spatial ability. Four modules are available with developmentally appropriate tasks for testing individuals with different language abilities: (1) "Preverbal/single words/simple phrases,” (2) “Flexible phrase speech,” (3) “Fluent speech child/adolescent,” and (4) “Fluent speech adolescent/adult (Lord et al., 2000).” Items are scored on a 4-point Likert-type scale from 0 (no abnormality) to 3 (moderate to severe abnormality), and fall into measures of either social affect (SA) or restricted repetitive behavior (RRB). Calibrated severity scores (CSS), standardized for age and language level, are calculated for SA items (CSS-SA) and RRB items (CSS-RRB), and a total ADOS-CSS is the sum of these scores. Children with a total ADOS-CSS score from four to five are classified as having "autism spectrum," and those with a score of six or more are classified as having “autism” (Gotham, Pickles, \& Lord, 2009; Gotham et al., 2008; Hus et al., 2014). The present research team performed standardization of module 4 scores to match prior standardization of modules 1-3 by mapping raw social affect and restricted, repetitive behavior scores onto total, social affect, and restricted repetitive behavior severity scores (V. Hus Bal, personal communication, March 24, 2017). 


\subsubsection{Analysis for Aim 1}

Analysis for the SSCSI factor analysis can be found in the instrumentation manuscript, Psychometric properties of the Simons Simplex Collection Sleep Interview (section 4). The ten dichotomous SSCSI items, SSCSI composite score and subscale scores, NVIQ, ADOS severity score, and sex were described using frequency counts and percentages. Regular sleep duration in hours per night and age in years were described using mean, standard deviation, and range. Outliers in regular sleep duration—reported sleep duration less than 3 standard deviations below the population mean-were replaced with the 3rd standard deviation below the mean (4.85 hours). Correlations between age, sex, NVIQ, ADOS severity score, GID, and seizures were compared with Student's t-test, chi-square, or Mann-Whitney U analysis. Descriptive and correlational analyses were performed with SPSS version 23 (SPSS Inc., Chicago, IL).

SSCSI composite sleep scores have been categorized into three levels of sleep problems: none/minimal (scores 0-1), mild (2-3), moderate/severe (> 4). Baseline and adjacent category logistic regression analyses were performed to explore and compare the association between SSCSI composite and factor scores, and child characteristics. This method uses maximum likelihood estimation to compare the log odds of being in one group versus another. Six independent variables were included: age, ADOS severity score (range: 4-10), sex (male=0, female=1), NVIQ $(<40=1,40-54=2,55-70=3,71-100=4$, $>100=5$ ), GID (no=0, yes=1), and seizures (no=0, yes=1). Where total ADOS severity score was significant, social affect and restricted, repetitive behavior severity scores were analyzed. Analyses were performed by building models with no sleep problems versus sleep problems (mild and moderate/severe) as the outcome variable, and then looking between sleep problem severities (none/minimal vs. mild, mild vs. moderate/severe, none/minimal vs. moderate/severe). The most parsimonious model in each case was chosen using the Akaike information criterion (AIC). Because of small proportions of children in the female, and middle and late childhood subgroups, only baseline (none/minimal sleep problems vs. sleep problems) analyses were performed in subgroups. Logistic regression analyses were done with the full sample, and then stratified by age group and sex. Logistic regression was performed with the MASS 
package in the R suite (R Core Team, 2016). Level of significance was set at $\mathrm{p}<0.05$. Power to detect factors associated with sleep patterns was calculated assuming 20 independent tests, resulting in a corrected significance level of 0.05/20. With over 2,600 subjects assessed, we will have good power ( $>80 \%$ ) to detect an effect of size 0.06 SD. Of the full sample, 2,619 have complete data on the ADOS, IQ scales, and SSCSI and were used for the analyses.

\subsubsection{Analysis for Aim 2}

\subsubsection{Logistic regression analysis}

The SSCSI items, composite score and subscale scores, and sex were described using frequency counts and percentages. Parent reported regular sleep duration (hours per night) and age (years) were described using mean, standard deviation, and range. Regular sleep duration less than 3 standard deviations below the population mean, were replaced with the 3rd standard deviation below the mean (4.85 hours). MannWhitney U was used to compare SSCSI composite and subscale scores between age groups, and chisquare analyses to compare between sexes. Descriptive and correlational analyses were performed with SPSS version 23 (SPSS Inc., Chicago, IL).

SSCSI composite and subscale scores were dichotomized into none/minimal (scores 0-1) or sleep problems present $(\geq 2)$. Logistic regression analyses were performed to estimate associations between SSCSI composite and factor scores, and individual variant reference allele dosage (0, 1, 2 alleles). HardyWeinberg equilibrium (HWE) was assessed in participants of non-Hispanic European Ancestry using the HardyWeinberg package in the R suite (R Core Team, 2016) and a $p<0.00023$ (Bonferroni correction). All variants were in HWE. All models were adjusted for age (years), ADOS severity score (range: 4-10), sex (male=0, female=1), NVIQ ( $<40=1,40-54=2,55-70=3,71-100=4,>100=5)$, GID (no=0, yes=1), seizures (no=0, yes=1), and ancestry (1=non-Hispanic European, 2=Asian, 3=Hispanic European, 4=African American). Analyses were performed by building models with no sleep problems versus sleep problems (mild or moderate/severe) as the outcome variable. Odds ratios with 95\% confidence intervals 
were used to measure the strength of associations between each variant and sleep patterns. Logistic regression was performed with the MASS package in the $\mathrm{R}$ suite. Level of significance was set at $p<0.00023$ (Bonferroni correction).

\subsubsection{Transmission disequilibrium test}

The transmission disequilibrium test (TDT) is one of several family-based association methods, a group of tests that examines how parental genotypes or alleles are transmitted to offspring affected by a disease or trait. The TDT tests Mendel's law of segregation, which states that parental alleles are randomly and independently transmitted to offspring, with each allele having a probability of transmission $=1 / 2$. According to the law of segregation, there should be no association between a marker allele and a diseasesusceptibility allele, because they will be transmitted independently. Transmission disequilibrium occurs when there is non-random or greater than expected transmission of a certain allele to offspring with a trait or disease (Laird \& Lange, 2011). Alleles transmitted to offspring are viewed as “cases,” and alleles not transmitted as "controls," and a significant TDT test statistic indicates both linkage and association between a marker and the disease susceptibility allele. One advantage of the TDT over traditional case/control studies, is that confounding variables are completely matched between cases and controls, since "case" and "control" alleles come from the same person. Thus, the test is robust to population stratification factors. The TDT also does not require the disease model to be identified, and the distribution of the trait or disease in the general population does not affect the validity of the test. A disadvantage is that only heterozygous parents provide the information necessary to perform the TDT, and thus information on homozygous parents is thrown away (Barmada, 2016).

The TDT, performed with parent-child trios using McNemar's test (McNemar, 1947), is a test of two binomial samples, in this case transmitted alleles and non-transmitted alleles. A contingency table is formed with transmitted and non-transmitted alleles, and a modified chi-square test assesses the null hypothesis that the probability that either allele will be transmitted is equal. A significant result would 
occur if one allele was transmitted significantly more often than would be expected by chance (Walker \& Shostak, 2010).

\subsection{STRENGTHS, POTENTIAL LIMITATIONS, AND ALTERNATE APPROACHES}

\subsubsection{Strengths}

The major strengths of the proposed project pertain to the study of ASD, and the innovative methods used. This study proposes to address a phenotype that is highly disruptive to families affected with ASD. It will be more efficient and cost-effective because of using secondary data from a large, well-phenotyped sample from across the United States. Since genotyping has already been performed, analysis of genotypes will be much less costly. This study made use of RNA-Seq and in situ genetic data to guide selection of the most valuable genetic variants. By performing factor analysis on the SSCSI, the research team endeavored to understand better its internal structure, sub-dimensions, and validity. While a partial genetic basis for sleep patterns is well-established, specific genetic variation and its effects are not wellunderstood, especially in the context of ASD. Finally, this research was a good predoctoral training vehicle for the student to learn the tools of quantitative genetic analysis, which will provide the knowledge and skills required to become an independent researcher. Together, this study lays the groundwork to understand from a biological perspective why and how sleep patterns are abnormal in children with ASD. With this firm foundation, it should be possible to build further studies and interventions aimed at making sleep patterns more typical, thereby improving the quality of life for individuals with ASD and their families. 


\subsubsection{Limitations}

The first aim of the study has several limitations. The primary limitation of the factor analysis study was unequal sizes between early, middle, and late childhood groups. This may skew the ability to detect a similar factor structure in the SSC sample, and in the three groups. Although the sample is large and from sites across the United States, it is predominantly non-Hispanic Caucasian (74.5\%), and had a slightly greater ratio of boys to girls (5.9:1) than that seen in the general ASD population (4.5:1). This could limit the ability to generalize results to other populations. However, this is the largest such sample in the world, and what we learn from it should have general value. Another limitation is that little was known about the origin and administration of the SSCSI, and no other sleep instruments were used, precluding the calculation of test-retest reliability, interrater reliability, and convergent validity. Without an objective measure of sleep, all items were based on parents' report, and subject to reporting bias. In addition, laying down with a child may be accepted in some cultures and families practicing co-bedding, and this was not taken into consideration. Finally, without also testing a control group of typically developing children, it is difficult to know what sleep problems are unique to ASD and which are common in all children of a certain age.

Several factors related to the second aim have the potential to decrease the effect of the study. First, using secondary data precludes measuring levels of hormones related to sleep patterns, like melatonin and cortisol. Conversely, studies such as this one require large samples making it almost impossible to muster such a sample for which hormones were measured directly. Second, as neither the CMC nor GTEx collected samples with an eye to timing of circadian gene expression, the choice of eQTLs may not capture markers that were not expressed at the time of sample collection. The CMC used samples from the brains of adults, which may exhibit different gene expression patterns than the brains of children. Third, it is possible that melatonin/SCN genes are not key to driving sleep patterns. If this is the case, the student will evaluate the developing literature on the biology and genetics of sleep patterns, and investigate other genes for future analyses. 


\subsubsection{Alternate approaches}

An alternative approach may have been taken in performing the exploratory factor analysis on sleep questions that would become the SSCSI. Enuresis ("wet the bed") and sleep disordered breathing (combined "difficulty breathing at night," “sleep disordered breathing,” and "sleep apnea”) have been shown to disturb sleep in TD children. Based on this evidence, these two items may have been forced into the factor analysis model. This means that the items would be kept in the model despite poor associations with other items in the model, lower internal consistency (Cronbach's $\alpha$ ) with the items in the model, and low factor loading scores. The items "wet the bed," "tonsils and adenoids present," and "sleep disordered breathing," all had small correlations with other sleep items $(r \leq 0.13)$. The communality-how much total variance in an item is explained by the extracted factors - was very low for "wet the bed" $(0.031)$. When "wet the bed" and "sleep disordered breathing" were forced into the factor model, three factors emerged for early childhood (previously referred to as pre-pubertal) and two factors emerged for middle and late childhood (previously referred to as pubertal and post-pubertal respectively), making interpretation and generalized used of the instrument very difficult. It was concluded that, although enuresis and sleep disordered breathing affect sleep in other samples, they may not have strong effects on sleep patterns in the SSC sample and were allowed to drop out of the model as factor analysis proceeded.

A second alternative approach that may have been taken when analyzing the SSCSI is to treat the SSCSI composite and subscale scores as continuous variables rather than categorizing them. This approach would have been less ideal than the categorical approach taken, as the scores are discrete—data may only take set, discrete values - rather than continuous data-data may take infinite small values between integers. This approach would also have been less ideal because the composite and subscale scores were very positively skewed. 


\subsection{PROTECTION OF HUMAN SUBJECTS}

This research analyzes existing genetic, phenotypic, and gene expression data. The genetic and phenotypic data are drawn from the Simons Foundation Autism Research Initiative (SFARI) database, an existing resource for autism research. This study was submitted, reviewed, and approved by the University of Pittsburgh Institutional Review Board (IRB) in October 2015 (PRO15050345, Appendix F).

\subsubsection{Potential risks for proposed secondary analysis}

The research team has received and analyzed de-identified data only. They do not have access to biological specimens or access to personal identifying information. The greatest risk to human subjects from data received by and analyzed at the University of Pittsburgh is theft. If the SFARI data were stolen, and if they were somehow associated with a specific person, this could cause embarrassment and discomfort to the subject who participated in the parent study.

There are no direct benefits to participants in this study. The research team will consider each aim of the project complete when we have answered the specific scientific questions. Eventually, such knowledge could benefit mankind. In summary, the data to be analyzed are de-identified. Thus, the study presents very little risk to the subjects. The general knowledge gained by the study could benefit mankind. We believe the risk/benefit ratio is acceptable.

\subsubsection{Procedures for protection against risk}

Because the parent study involved risks greater than risks experienced in activities of daily living, informed consent was received from parents and assent was received from children as required by each site’s local IRB. In addition, each participant was assigned Global Unique Identifiers (GUID) produced from created from personal information using an algorithm. Each site maintained personal identifiers on 
site, and only deidentified GUIDs were sent to the central database. At no times does the present research team have access to personal identifiers. Although it would be extremely difficult, it may be possible to deduce personal identities from demographic and genotype data. For this reason, all data will be retained behind the UPMC firewall, which is formidable, has tight limits on remote access, and is monitored for security at all times. Data is never shared with individuals outside the present research plan.

The student has received exempt approval and will maintain approval from the University of Pittsburgh IRB for research on de-identified data from human subjects. She will report any data theft directly to the University of Pittsburgh IRB. 


\subsection{SUMMARY OF STUDY}

\subsection{PRELIMINARY WORK ON DISSERTATION STUDY}

The purpose of this research was to: (1) examine the relationship between sleep patterns and characteristics of children with ASD—age, sex, ASD severity, IQ, and medical conditions.; and (2) determine if there are genetic associations between 27 melatonin/SCN genes and sleep patterns in children with ASD. Genetic variants were chosen for association analysis based on their impact on gene expression, as measured by RNA-Seq and SNP data. The results for the SSCSI psychometrics are presented in the instrumentation manuscript, "Psychometric properties of the Simons Simplex Collection Sleep Interview.” Results for Specific Aim 1 are presented in the data-based manuscript, "Characteristics of sleep in children with autism spectrum disorders from the Simons Simplex Collection.” Results for Specific Aim 2 are presented in the data-based manuscript, "Variations in genes related to sleep patterns in children with autism spectrum disorder.”

\subsection{PROPOSAL CHANGES}

An attempt was made to capture normal shifts in sleep patterns throughout childhood by exploring puberty stage as a predictor of sleep patterns. Because the average age of puberty in the United States is between 8-13-years for girls (mean age of menarche is 12.2-12.8-years) and 9-14-years for boys (AlSahab et al., 2012; Cabrera et al., 2014), children in the SSC sample were divided by age into three bands 
called pre-pubescent (4-8-years), pubescent (9-13- years), and post-pubescent (14-18-years). The puberty development scale was developed by the parent study and relied on the opinion of the medical professional performing the exam. The scale measured 5 items in males (body hair, facial hair, growth spurt, skin changes, voice changes) and 4 in females (body hair, breast changes, growth spurt, skin changes) on a scale from 1 to 4 (1 = "has not begun,” 2 = "barely started,” 3 = ”definitely underway," 4 = ”seems complete”). Females also had one item, "begun period” measured on a two-point scale. Because the scale was ambiguous (ex. "seems complete") and could be interpreted differently by each medical professional who used it, all children had data for age, but many did not have data for puberty, and because results using the scale will not be generalizable, it was decided to use age instead of puberty level in analyses. The instrumentation manuscript uses the terms "pre-pubertal," “pubertal," and "postpubertal” even though these groups were determined using age. The terms "early childhood," "middle childhood," and "late childhood” were used in the two subsequent manuscripts.

After review of recent literature and consideration of other variables that may affect sleep patterns, GID and seizures were added as independent variables. Presence of GID was defined as parent reports of bloating/excess gas, celiac disease, constipation, diarrhea, ulcers, gastroesophageal reflux disease, inflammatory bowel disease (Crohn's disease, ulcerative colitis), irritable bowel syndrome, abdominal pain, unusual stools, vomiting, and/or other GID; and seizures as parent reports atonic drop, infantile spasms; or partial complex, grand mal, petit mal, simple partial, and/or other seizures. Presence or absence of GID and seizures was determined using data from the medical history interview conducted by the parent study at baseline.

The transmission disequilibrium test was added to the Aim 2 analysis to reinforce results from the logistic regression from a different angle. The logistic regression analysis of genetic variants on SSCSI sleep scores looked at associations between the number of reference alleles possessed by the participant and their sleep score, while the TDT examined if there was over-transmission of one allele to children with sleep problems. 


\subsection{CONCLUSIONS, IMPLICATIONS FOR NURSING, AND FUTURE STUDIES}

Several conclusions may be drawn from this study. As expected, many sleep problems were most prevalent in early and middle childhood, suggesting that treatments may need to be tailored toward sleep problems evident in younger children. These included bedtime problems and the need for a parent to lay down with the child, difficulty falling asleep, and frequent/prolonged awakenings. However, children in middle childhood had more difficulty waking than those in early and late childhood, and children in late childhood had more excess daytime sleepiness than younger children, implying that treatments will need to evolve as children age.

Using the SSC sample, this study confirmed numerous prior reports of sleep problems in children with ASD. These reports reinforce the need for health care professionals to screen for and discuss sleep problems with their ASD patients and families. As recommended by Malow et al. (2014), parental education in proper sleep habits should be provided. Use of the SSCSI may be used to screen for sleep problems in children with ASD in primary care and sleep medicine clinics, as well as in a range of specialty clinics—neurology, pulmonology, cardiology, behavioral medicine, etc. This screening may aid in proper diagnosis and treatment of other symptoms, and contribute to holistic, multispecialty care. In addition, as sleep has been associated with behavioral, cognitive, and biological variables, the SSC provides a wealth of opportunities to incorporate sleep into research in this large, well-characterized sample of children with ASD. The SSCSI may be used by such researchers to incorporate sleep patterns into studies focusing on a wide range of variables.

This study advances the understanding of sleep and ASD by showing that presence of GID, seizures, young age, and low NVIQ increase the odds of having sleep problems. As other studies have found, nearly half of children in this sample were reported to have GID, and these gastric symptoms were significantly related to sleep problems. The current study additionally found that rates of GID and its relationship to sleep problems were not different between age groups or sexes. Seizures were found to be significantly associated with composite sleep problems and nighttime problems, but not daytime problems 
or sleep duration problems, which may imply that seizure activity impacts length, depth, and/or microarchitecture of sleep patterns. That low NVIQ (more severe symptoms), but not ASD severity, is related to increased composite sleep problems is intriguing and will require more investigation to explain. These results, however, emphasize that practitioners and researchers from many specialties must be attentive for sleep problems in their ASD populations.

The second aim of this study attempted to determine if there are genetic associations between melatonin/SCN genes and sleep patterns using association analysis and transmission disequilibrium tests. Variants were chosen because of their significant effect on expression of target genes, primarily in the brain. However, genetic analysis of the 72 eQTLs and SNPs did not demonstrate association with sleep problems in this sample, indicating that variation in expression, or transcription, of genes in the melatonin/SCN pathway is likely not a driver of sleep problems. If transcription is not driving sleep problems, a number of research questions now follow, including the role of post-translational modification of melatonin/SCN proteins and epigenomic mechanisms in sleep problems, and the contribution of other sleep homeostasis gene pathways. In addition, the innovative methods used in these genetic analyses demonstrate a method that may be employed by other research groups interesting in the use of gene expression for association analyses.

Future research could proceed in several directions. Use of a validated instrument adds a measure of confidence to a study and is essential for collection and interpretation of accurate patient data. However, due to the data available, a full description of the reliability and validity of the SSCSI was not possible. Before the SSCSI is used in full-scale studies, it must be tested in other samples of children and adolescents with ASD in order to establish test-retest reliability, inter-rater reliability, and to support our findings of internal consistency. The SSCSI must also be used along with validated sleep questionnaires such as the Children's Sleep Habits Questionnaire, Modified Simmons and Parraga Sleep Questionnaire, or the Clinical Global Index-Sleep in order to establish convergent validity. In addition to refining the SSCSI, use of objective measures of sleep, such as actigraphy and polysomnography, would provide a strong foundation for association studies. As a next-step toward translation of research results into clinical 
practice, evaluation of screening for sleep problems in various clinical settings may be performed to document how often healthcare providers address sleep problems.

Continued research into the genomics of sleep problems in individuals with ASD are clearly needed. The lack of association found between expression of genes and sleep patterns in the present study does not imply that the melatonin synthesis and SCN pathway should be abandoned. Although this study showed that transcription of these genes was not influencing sleep patterns, the effect of translation and post-translational modification on melatonin and SCN proteins cannot be dismissed. That a number of research studies have shown low melatonin or melatonin metabolite levels demonstrates this is still a valid research area. Expanding on recent research suggesting that risk for ASD may lie in common variants acting additively, it may be informative to investigate risk haplotypes from melatonin-synthesis genes or SCN genes as guided by literature and well-thought out hypotheses. However, as other gene pathways in the brain and body, such as glutamate, GABA, and serotonin are known to interact with melatonin and the SCN, inquiries should not be limited to the melatonin/SCN pathway. In summary, future research into the biological basis of sleep problems in ASD—genetic, biochemical, comorbid conditions - and the effect of therapies on sleep problems may move this research into the realm of clinical interventions to improve quality of life for families affected by ASD and sleep problems. 


\title{
3.0 INSTRUMENTATION MANUSCRIPT: PSYCHOMETRIC PROPERTIES OF THE SIMONS SIMPLEX COLLECTION SLEEP INTERVIEW
}

\author{
3.1 ABSTRACT \\ Background and Purpose: An estimated $40-80 \%$ of children with autism spectrum disorders (ASD) \\ have sleep problems. The Simons Simplex Collection Sleep Interview (SSCSI) is a parent-report \\ questionnaire assessing bedtime and nighttime sleep problems, and daytime function. The present study \\ evaluated the factorial model of the SSCSI that best characterizes children 4-18 years with ASD. \\ Methods: Exploratory factor analysis was performed using principal component analysis and promax \\ rotation, beginning with 16 items and ending with 10 items. \\ Results: Exploratory factor analysis concluded with ten dichotomous items, plus age and regular sleep \\ duration, in three factors: nighttime problems, daytime problems, and sleep duration problems. The \\ analysis was performed on the full sample, and on prepubertal (4-8-years), pubertal (9-13-years), and \\ postpubertal (14-18-years) subgroups. \\ Conclusion: Further refinement, including confirmatory factor analysis, test-retest reliability, and \\ convergent validity testing are needed.
}




\section{$3.2 \quad$ INTRODUCTION}

Autism spectrum disorder (ASD) encompasses a range of pervasive neurodevelopmental disabilities that begin early in childhood and last throughout life. Although all children require adequate sleep for normal growth and development (Hirshkowitz et al., 2015; Iglowstein, Jenni, Molinari, \& Largo, 2003), an estimated 40-80\% of children with ASD have comorbid sleep problems (May et al., 2015; Veatch et al., 2016). These sleep problems may include increased sleep latency (time to fall asleep), decreased sleep efficiency (percentage of time asleep to time in bed), parasomnias (e.g. night terrors, sleep walking), and nighttime or early morning waking (Elia et al., 2000; Galli-Carminati, Deriaz, \& Bertschy, 2009;

Goldman et al., 2010; Liu et al., 2006; Malow et al., 2006).

Nighttime behaviors and abnormal sleep duration in children with ASD can be very disruptive to the rest of the family, and often decrease the family’s quality of life (Giallo, Wood, Jellett, \& Porter, 2013; Johnson \& Simpson, 2013; Schwichtenberg et al., 2013). Parents of children with ASD report significantly more and longer night wakings than parents of typically developing children (Krakowiak et al., 2008; Souders et al., 2009). Parents often must get up to help the child settle and fall asleep again, or to ensure the child is safe while awake. These wakings may also disrupt other siblings who sleep in the same room or near the child with ASD, possibly affecting their daytime function. Parental sleepiness during the day can affect family functioning, work productivity, and even public safety (Giallo et al., 2013; Goldman, Bichell, Surdyka, \& Malow, 2012; Tietze, Zernikow, Michel, \& Blankenburg, 2014). Behavior problems in the child with ASD are exacerbated by their lack of quality sleep, and may disrupt classroom functioning at school (Allik et al., 2008; Schwichtenberg et al., 2013).

Existing sleep instruments, originally designed for use with typically developing children, have been adapted for use in the ASD population. The most commonly used instrument is the Children's Sleep Habits Questionnaire (CSHQ; Owens, Spirito, \& McGuinn, 2000), a 45-item, parent-report measure of sleep behaviors that has been validated in children from preschool through school-age (Goodlin-Jones, Sitnick, Tang, Liu, \& Anders, 2008; Markovich, Gendron, \& Corkum, 2015). Eight subscales within the 
CSHQ are bedtime resistance, sleep onset delay, sleep duration, sleep anxiety, night wakings, parasomnias, sleep disordered breathing, and daytime sleepiness. Items are scored on a three-point Likert-type scale with the behavior occurring in the previous week "usually" (5 to 7-times/week), “sometimes” (2 to 4-times/week), or “never” (zero to one-time/week). Internal consistency in a community sample of healthy, typically developing children 4 to 10-years-old was adequate for the full scale $(a=0.68)$, and acceptable for the subscales $(a=0.36-0.70)$. Test-retest reliability of subscales was satisfactory, ranging from r=0.62-0.79 (Owens et al., 2000). Internal consistency was similar in a sample of 2 to 16-year-old children with ASD ( $a=0.68$, Johnson, Turner, Foldes, Malow, \& Wiggs, 2013).

The Simonds and Parraga Sleep Questionnaire (Simonds \& Parraga, 1982) is a similar instrument originally developed to screen for sleep problems in typically developing children 5-18-years-old, but modified and validated in children with ASD. The Modified Simonds and Parraga Sleep Questionnaire (MSPSQ; Johnson et al., 2013; Wiggs \& Stores, 1996) is a 36-item, parent-report questionnaire consisting of 7 subscales scored on a 5-point Likert-type scale, with higher scores denoting more problems: bedtime resistance, sleep onset delay, sleep anxiety, night waking, parasomnia, sleep disordered breathing, and daytime sleepiness. In a sample of 345 children with ASD, the MSPSQ had good overall internal consistency $(\alpha=0.80)$, and test-retest reliability after 2 weeks ( $r=0.83$; Maas et al., 2011). However, in a follow-up study, internal consistency of subscales was lower, ranging from 0.19 to 0.66 (full scale $\alpha=0.67$; Johnson et al., 2013).

To complement parent-report instruments and objective measures, such as actigraphy (a movement sensor used to determine sleep and wake patterns) and polysomnography (in-laboratory sleep study), Malow et al. (2016) developed the clinician-report Pediatric Sleep Clinical Global Impressions Scale (CGI-S) for use in children with ASD. Based on components of insomnia criteria from the International Classification of Sleep Disorders (American Academy of Sleep Medicine, 2014), the CGI-S queries bedtime problems—ability to fall asleep and stay asleep independent of parents, bedtime resistance, sleep onset delay_night wakings, parents' satisfaction with the child's sleep patterns, and the clinician's general opinion about the child's sleep. Items are presented in two sections: current problems 
and improvement from previous assessment. Responses are rated by the clinician on a 7-point Likert-type scale ranging from "no concerns” (1) to "highest level of concerns seen by the examiner" (Malow et al., 2016, p. 371). In pilot testing, significant associations between the CGI-S and CSHQ were observed in sleep onset delay ( $r=0.65, p<0.001)$, night wakings ( $r=0.40, p<0.001)$, and overall score $(r=0.29, p<$ 0.001). Sleep onset delay score on the CGI-S was also significantly associated with objective actigraphic measure of sleep onset latency (time required to fall asleep; $r=0.75, p<0.01$ ).

The Simons Foundation Autism Research Initiative began in 2003 with the goal of improving the scientific understanding of ASD. One of their major projects was the Simons Simplex Collection (SSC), a sample of approximately 2,750 simplex families (only one child with ASD, termed the proband) from 12 sites in North America. A main goal of the SSC was to identify genetic variants associated with ASD risk (Fischbach \& Lord, 2010). Its data have been used to study genomics of cognition and behavior in ASD (Frazier et al., 2014; Gamsiz et al., 2013; Green et al., 2016), the broad autism phenotype (Davidson et al., 2014), the impact of copy number variants on risk for ASD (Desachy et al., 2015; Sanders et al., 2015; Webb et al., 2016), and medical comorbidities with ASD (Gorrindo et al., 2012; Viscidi et al., 2013).

More than thirty instruments were used with the SSC to collect a number of variables, including family health; psychiatric, developmental, and environmental exposure histories; proband medical, developmental, treatment, school, and ASD histories; and current proband comorbidities, intelligence, behavior, motor skills, and speech. Blood samples were taken from biological parents, the proband, and one sibling, if available, for genomic study. Probands were strictly phenotyped through parent interview, medical history, and multiple well-validated instruments (Fischbach \& Lord, 2010).

\subsubsection{Study aims}

As one of the largest cohorts of children with ASD currently available, the SSC presented a good opportunity for the study of sleep throughout childhood in children with ASD. The Simons Simplex Collection Sleep Interview (SSCSI) originated as part of the full medical history performed upon entrance 
to the study, and consisted of 18 questions about sleep habits, including bedtime behavior, nighttime sleep patterns, and daytime function. The SSCSI has not previously been evaluated for reliability and validity, or undergone factor analysis. The purpose of this study was to (1) evaluate the factor structure that best characterizes the SSC sample, and (2) establish validity and reliability of the SSCSI in this sample.

\subsection{METHODS}

\subsubsection{Participants}

Families were recruited from clinics at academic medical centers serving children with ASD, as well as from service providers, parent and advocacy groups, web postings, and radio and television advertisements. Children were included if they were between 4- and 18-years-old; met the criteria for ASD based on the Autism Diagnostic Observation Schedule [ADOS], Autism Diagnostic InterviewRevised [ADI-R], and Diagnostic and Statistical Manual of Mental Disorders-IV-Text Revised [DSM-IVTR]; and had a non-verbal mental age of at least 24-months for probands 4- to 7-years-old, or 30-months for probands $\geq 7$-years-old. Probands were excluded if they were born at less than 36 -weeks and had a birth weight less than 2,000 grams; were born with a significant injury, abnormality, or disorder effecting the brain; had extended prenatal or birth complications; had fragile X or Downs syndrome; had sensory or motor disorders that would confound diagnostic measures; had a history of severe nutritional or psychological deficiency; were not fluent in English; or had another family member affected with ASD up to third degree relatives of the proband. One sibling was included in the study if available (Simons Foundation Autism Research Initiative, 2014). Because notable shifts in sleep patterns occur around puberty, the sample for the present study was divided into three age groups: prepubertal (4-8-years), pubertal (9-13- years), and post-pubertal (14-18-years). 


\subsubsection{Procedure}

This study was a secondary analysis of existing data from the Simons Foundation. Twelve study sites across the United States and Canada collaborated with the Simons Foundation Autism Research Initiative on the SSC. The SSCSI was completed at a clinic visit or by telephone interview of parents. Procedures of the parent study and characteristics of the SSC parent study sample have been described previously (Fischbach \& Lord, 2010).

Demographic data—age, ethnicity, family income, living situation, parental education—were collected at the time of initiation in the study, and the SSCSI was administered within three months of study initiation. Children with ASD were strictly phenotyped by trained clinicians using the Autism Diagnostic Interview-Revised (ADI-R; Lord, Rutter, \& Le Couteur, 1994), Autism Diagnostic Observation Schedule (ADOS; Lord et al., 2000), and multiple neurocognitive and behavioral measures described previously (Fischbach \& Lord, 2010; Simons Foundation Autism Research Initiative, 2014). Phenotypic data was acquired for the present study from the Simons Foundation software platform, SFARI Base. Incentives were not offered to participants. For the parent study, informed consent was obtained from all parents or caregivers included in the study, and assent from children as required by local institutional review boards. Approval was obtained from the University of Pittsburgh (Pittsburgh, PA) Institutional Review Board for the present study.

\subsubsection{Survey instrument}

The original questionnaire included 18 items about going to bed and falling asleep; waking during the night; sleepwalking or nightmares; wetting the bed; breathing difficulties at night; waking in the morning; daytime sleepiness; regular bedtime, wake time and duration; age in months at questionnaire completion; and regular duration of sleep in hours. When a range of hours was given, the mean was taken for use in this analysis. Of the 18 items, 16 were dichotomous (yes/no) pairs inquiring if this sleep problem had ever 
occurred, and if it is currently occurring. In considering if both "ever" and "currently" items should be retained, it was decided to only use "currently" items. One reason for this is that older children would have a much longer time to have had these sleep problems than younger children, and so may appear to have an inflated number of sleep problems. In addition, problems like wetting the bed or sleepwalking, which may be more developmentally typical at younger ages, are “currently” abnormal in older children. Only the 16 dichotomous items were analyzed in the present study. A continuous composite score is made by summing items as 0 for “no” and 1 for “yes,” and adding 1 point for sleep duration outside National Sleep Foundation recommendations for the child's age (Hirshkowitz et al., 2015). Participants were excluded from the present study if more than two items were missing or coded as unknown. No information is available on the original writers or development process of the original sleep questions.

\subsubsection{Measures}

ASD severity was measured with the Autism Diagnostic Observation Schedule (ADOS), a semistructured observational instrument used with other instruments for diagnosis of ASD and classification of severity. The measures that make up the ADOS total score are operationalized as characteristics of social responsiveness, communication, language delay, and spatial ability. Four modules are available with developmentally appropriate tasks for testing individuals with different language abilities: (1) "Preverbal/single words/simple phrases,” (2) “Flexible phrase speech,” (3) "Fluent speech child/adolescent,” and (4) “Fluent speech adolescent/adult (Lord et al., 2000).” Items are scored on a 4point Likert-type scale from 0 (no abnormality) to 3 (moderate to severe abnormality), and fall into measures of either social affect or restricted repetitive behavior. Calibrated severity scores (CSS), standardized for age and language level, are calculated and a total ADOS-CSS is the sum of these scores (Gotham et al., 2009; Gotham et al., 2008; Hus et al., 2014). ADOS-CSS was used in this study as an indicator of ASD severity. 
Four instruments-Weschler Intelligence Scale for Children-IV, Weschler Abbreviated Scale of Intelligence, Differential Abilities Scales-II, and Mullen Scales of Early Learning - were used to quantify IQ in the SSC sample depending on chronologic and mental age. Each scale produces standardized verbal IQ (VIQ), nonverbal IQ (NVIQ), and full-scale IQ (FSIQ) scores that can be used to measure and compare IQ across instruments. The Differential Ability Scales-II (DAS-II) Early Years is divided into a lower level for children with mental age 2 years 6 months to 3 years 5 months, and an upper level for children with mental age 3 years 6 months to 6 years 11 months; and the DAS-II School Age is used for children with mental age 7 years to 17 years 11 months (Elliott, 2012; PsychCorp, 2016). The Weschler Intelligence Scale for Children-Fourth Edition (WISC-IV) is a standardized intelligence test used in children with mental age 6 years to 16 years 11 months (P. E. Williams et al., 2003). The Wechsler Abbreviated Scale of Intelligence (WASI) is a standardized intelligence test used in individuals with mental age 6 years to 90 years 11 months (Axelrod, 2002; Canivez et al., 2009; Goldstein \& Mazefsky, 2013; McCrimmon \& Smith, 2012). The Mullen Scales of Early Learning (MSEL) is a standardized cognitive test used with children from birth to a mental age of 5 years 8 months (Bradley-Johnson, 1997).

\subsubsection{Statistical analysis}

Descriptive statistics of the data were calculated, including frequencies of yes/no responses for the 16 dichotomous items. Means with standard deviations, and ranges were computed for age and sleep duration. No ages were missing. Subjects missing regular sleep duration were retained $(n=30)$, and other missing values were excluded pairwise. Histograms were generated for the continuous variables, including calculations of skewness and excess kurtosis, and the Shapiro-Wilkes test was performed to assess for normality. Because the questions, "regular bedtime and wake time,” "regular sleep duration,” and "sleep duration is enough" were worded positively (“yes” means no problem), these items were reverse coded to match the other dichotomous items. 
Subsequently, the characteristics of the data were assessed, beginning with inter-item Spearman correlations and significance levels, and the presence of item clusters. Items were assessed for violation of factor analysis assumptions: redundancy of items, excessive inter-item correlations $(r>0.9)$, sampling inadequacy, and outliers (Tabachnick \& Fidell, 2013a). Redundancy was explored particularly among the five breathing-related questions (“difficulty breathing at night," "have the tonsils been removed," "have the adenoids been removed," "has a physician diagnosed obstructive sleep apnea," and "child snores more than half the time”). Bartlett's Test of Sphericity was performed to test the null hypothesis that there was no correlation between items (significance: $p<0.05$ ). The overall Kaiser-Meyer-Olkin $(\mathrm{KMO})$ test determined if the sample size was adequate relative to the number of items. The cutoff of KMO $>0.6$ was used (Tabachnick \& Fidell, 2013b). Measures of sampling adequacy (MSA) assessed the strength of correlations among individual items.

Exploratory factor analysis was attempted with principle components analysis on the full scale and by age group. The Spearman correlation matrix was specified for the analysis, given the dichotomous nature of the items. Rotation was then attempted to improve factor loading. Factors were determined using percent of variance explained by the model and scree plots. Where cross loading (an item loading nearly equally on several factors) was present, the item was placed in the factor that best fit it conceptually. Scale reliability and validity were assessed using Cronbach’s alpha, inter-item correlations, and statistics if item deleted. The scale was not administered more than once with this sample, so that testretest reliability was not calculated. The families involved in the parent study did not complete any other sleep questionnaires, precluding the examination of criterion-related validity. SPSS version 23 was used for analysis, and significance was defined as a $p$-value $<0.05$. 


\subsection{RESULTS}

\subsubsection{Sample characteristics}

Table 2 provides sample characteristics. Of the full sample $(\mathrm{N}=2,745), 2,737$ children had sufficient data on the SSCSI to be used for the analysis. Those excluded were not significantly different in age, ADOSCSS, VIQ, NVIQ, FSIQ, regular sleep duration, or sex. There were significant differences between those included and those excluded in the ADOS module used (Fisher's exact test, $p<0.05$ ). Among the 2,737 children included in analyses, no significant difference was found in the number of males or ADOS-CSS score between age groups. As would be expected, sleep duration was significantly different between groups (prepubertal vs. pubertal $d=0.10, p<0.01$; pubertal vs. postpubertal $d=0.52, p<0.01$ ), with mean duration in hours decreasing from pre- to post-pubertal. Mean sleep duration for each age group was within the National Sleep Foundation's recommendations for that age group (Hirshkowitz et al., 2015). NVIQ, as measured on age appropriate scales, had a significant decrease from the prepubertal to pubertal age group ( $d=0.01, p=0.03)$. Scores in all age groups were low compared to the general population mean of 100 (SD = 15). Neither age nor sleep duration were normally distributed. The distribution of age was considerably positively skewed, with the majority of children in the younger age range (range 4-18 years; $p<0.01$ ). The distribution of sleep duration was negatively skewed, with most children sleeping in the upper range of hours (range 3-13 hours per night; $p<0.01$ ), and 12 outliers were present with regular sleep duration ranging from 3 to 5 hours per night. These cases were assessed for influence as analyses proceeded. 
Table 2: SSCSI Sample Characteristics

\begin{tabular}{|c|c|c|c|c|}
\hline & $\begin{array}{c}\text { Full Scale } \\
\text { (4-18 years) } \\
\text { N=2737 }\end{array}$ & $\begin{array}{c}\text { Prepubertal } \\
\text { (4-8 years) } \\
n=1533\end{array}$ & $\begin{array}{c}\text { Pubertal } \\
\text { (9-13 years) } \\
\text { n=883 }\end{array}$ & $\begin{array}{c}\text { Postpubertal } \\
\text { (14-18 years) } \\
n=321\end{array}$ \\
\hline $\begin{array}{l}\text { Age [mean(SD)] } \\
\text { (years) }\end{array}$ & $9.0(3.6)$ & $6.4(1.5)$ & $11.1(1.4)$ & $15.8(1.1)$ \\
\hline Male [n(\%)] & 2302 (83.9) & 1285 (86.8) & 749 (87.2) & 268 (83.8) \\
\hline \multicolumn{5}{|l|}{ IQ [mean(SD)] } \\
\hline Full-scale & $81.2(27.9)$ & $82.0(25.7)$ & 80.1 (29.8) & $80.2(32.2)$ \\
\hline Verbal & $77.1(31.0)$ & $79.0(28.0)$ & $76.2(33.7)$ & $69.1(36.3)$ \\
\hline Nonverbal & $84.1(26.1)$ & $85.7(24.2)^{a} *$ & $83.0(27.9)^{\text {a } *}$ & $77.8(29.4)$ \\
\hline $\begin{array}{l}\text { ADOS } \\
\text { calibrated } \\
\text { severity } \\
\text { score[mean(SD)] }\end{array}$ & $7.4(1.7)$ & $7.5(1.6)$ & $7.4(1.8)$ & $7.3(1.8)$ \\
\hline $\begin{array}{l}\text { Sleep duration } \\
\text { hours } \\
\text { [mean(SD)] }\end{array}$ & $9.2(1.3)$ & $9.6(1.2)^{a * *}$ & $\begin{array}{r}9.0(1.2)^{\mathrm{a} * *} \\
\mathrm{~b} * *\end{array}$ & $8.4(1.1)^{b * *}$ \\
\hline
\end{tabular}

ADOS: Autism Diagnostic Observation Scale (range: 1-10); ${ }^{a}$ significant difference between prepubertal and pubertal groups; ${ }^{\mathrm{b}}$ significant difference between pubertal and postpubertal groups; * $p<0.05 ; * * p<0.01$

Prepubertal children were significantly more likely than pubertal children to need a parent to lay down with them, have frequent or long awakenings, wet the bed, and have tonsils and adenoids present; but less likely to have trouble falling asleep or difficulty waking. Pubertal children were significantly more likely than post-pubertal children to have trouble going to bed, need a parent to lay down with them, and wet the bed; and less likely to have sleep disordered breathing (all $\mathrm{p}<0.05$ ).

\subsubsection{Item reduction}

The characteristics of matrices—correlations, significance levels, and the determinant-describe how well individual items go together accounting or not accounting for the other items. Most dichotomous items had low to modest inter-item correlations $\left(r_{s} \leq 0.286\right)$. An item cluster with moderate correlations 
(0.340-0.517; $p<0.01)$ was seen between "difficulty going to bed," "need to lay down with child," and “difficulty falling asleep.” A second item cluster with more modest, but still significant, correlations $\left(r_{s}=\right.$ 0.267-0.302, $p<0.01$ ) consisted of “difficulty waking," “daytime sleepiness,” and "long/frequent naps.” These are items that were expected to cluster, and likely factor together.

After examining correlations and their significance levels, it was determined that not all three items related to breathing problems were necessary. "Sleep apnea” had non-significant correlations with about half of the other items in the instrument; and "snoring” and "difficulty breathing” with about a quarter of items. Because sleep disordered breathing is an umbrella term that could describe sleep apnea, difficulty breathing at night and snoring, the three items were collapsed into a single combined "sleep disordered breathing” item. "Wet the bed," "tonsils and adenoids present," and "sleep disordered breathing," each have small correlations with other items $\left(r_{s} \leq 0.126\right)$. These items were carefully evaluated during factor analysis for retention in the model.

\subsubsection{Model 1}

Factor analysis was attempted in the full sample by principle components analysis. In this initial model, communalities - preliminary estimates of how much total variance in each item was explained by the factors to be extracted - ranged from 0.174 (“wet the bed”) to 0.607 (“difficulty falling asleep”). Four factors had eigenvalues greater than 1.0, and were obviously above the elbow on the scree plot (Figure 5). However, these four eigenvalues only explain $44 \%$ of total variance, and 10 factors were required to explain $80 \%$ of the total variance. There was an uneven distribution of items in the factor matrix, with two factors having only one item each, and another factor having 8 items. There were no items with factor loadings greater than 0.675 , and "sleep walk/nightmares" did not achieve a factor loading $>0.4$. Promax rotation did not result in much improvement, with “wet the bed” and "sleep walk/nightmares” crossloading on two factors, and "regular duration is not enough" and "frequent/prolonged awakenings" having 
lower loading scores than unrotated items. Rotation does not change communalities or variance explained by factors, hence not improving these aspects of the model.

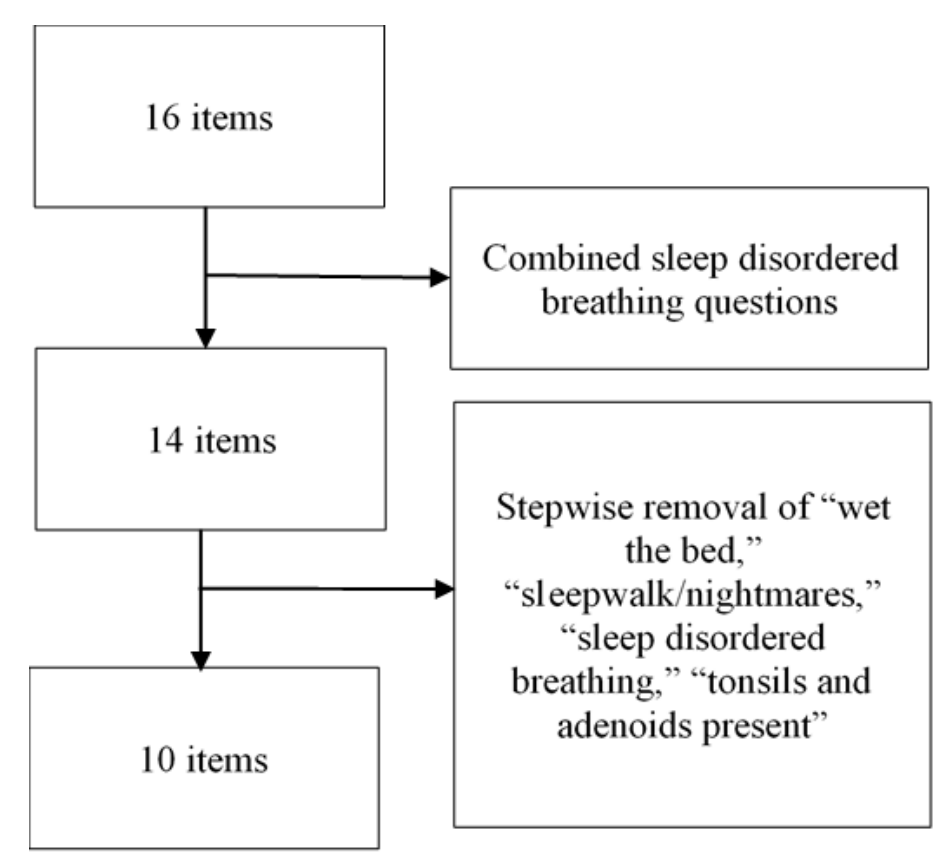

Figure 4: Flowchart of Dichotomous Item Reduction

\subsubsection{Model 2}

Because of the less-than desirable factor loadings seen in Model 1, a second model was attempted, again specifying a Spearman correlation matrix. Items were removed from the model in a step-wise fashion based on low or non-significant correlations, communalities, strength of factor loadings, and if Cronbach's $\alpha$ increased after deletion of the item (Table 3; Figure 4). For example, correlations between "wet the bed" and other items were very low (0.09) and non-significant for five items. Communality and factor loading were moderate, but Cronbach's $\alpha$ increased by 0.17 when "wet the bed" was removed. 
Before considering removal of this item, correlations were checked in the pre-pubertal age group, since this was the group most likely to experience wetting the bed. Correlations were found to be nonsignificant for ten of the fourteen items, so "wet the bed” was removed from the model. In the same way, “tonsils and adenoids present,” “combined sleep disordered breathing,” and "sleepwalk/nightmares” were removed until all items loaded on factors, Cronbach's $\alpha$ was no longer larger if items were deleted, and correlations were all significant, except for one pair ("awake frequently" and "irregular sleep duration;" $p=0.148$ ). The largest correlation among items was modest--0.499 between “difficulty going to bed” and “difficulty falling asleep.”

Table 3: Stepwise Removal of Questionnaire Items

\begin{tabular}{|c|c|c|c|c|}
\hline $\begin{array}{l}\text { Variables } \\
\text { removed }\end{array}$ & KMO & Cronbach's $\alpha^{*}$ & $\alpha$ if item is deleted & Factors in model \\
\hline [Full model] & 0.775 & 0.671 & $\begin{array}{l}\text { Wet the bed: } 0.688 \\
\text { Tonsils and adenoids } \\
\text { present: } 0.680\end{array}$ & 4 \\
\hline Wet the bed & 0.777 & 0.687 & $\begin{array}{l}\text { Tonsils and adenoids } \\
\text { present: } 0.698 \\
\text { Sleepwalk/ } \\
\text { nightmares: } 0.688\end{array}$ & 4 \\
\hline $\begin{array}{l}\text { Tonsils and } \\
\text { adenoids present }\end{array}$ & 0.772 & 0.698 & $\begin{array}{l}\text { Sleep disordered } \\
\text { breathing: } 0.702 \\
\text { Sleepwalk/ } \\
\text { nightmares: } 0.694\end{array}$ & 3 \\
\hline $\begin{array}{l}\text { Sleep disordered } \\
\text { breathing }\end{array}$ & 0.771 & 0.702 & $\begin{array}{l}\text { Sleepwalk/ } \\
\text { nightmares: } 0.700\end{array}$ & 3 \\
\hline $\begin{array}{l}\text { Sleepwalk/ } \\
\text { nightmares }\end{array}$ & 0.766 & 0.699 & $\begin{array}{l}\text { None higher than full } \\
\text { scale } \alpha\end{array}$ & 3 \\
\hline
\end{tabular}


As most bivariate correlations were significant with a 2-tail, 95\% confidence interval, oblique rotation (Promax with Kaiser normalization) was attempted. In this second, rotated model, communalities were slightly better, ranging from 0.291 (“frequent/prolonged awakenings”) to 0.686 (“irregular sleep duration”). Three factors had eigenvalues greater than 1.0, and could explain just over $50 \%$ of the variance (Figure 6). "Regular sleep duration is not enough" and "difficulty waking in the morning" switched factors when the model was rotated (Table 4). The factors will be known as night time problems, daytime problems, and sleep duration problems. Although "regular duration is not enough" seems like it should load with the other sleep duration problems, instead of with daytime problems, it may be that this item, conceptually, reflects the effect of not enough sleep on daytime function.

Figures 7-11 show the distribution of the ten extracted items for the full scale, and three subscales. Cronbach's alpha for the full, 10-item scale was $\alpha=0.694$ showing modest internal consistency, although internal consistency within the three factors (bedtime problems, daytime problems, sleep duration problems) was lower ( $\alpha=0.421-0.643$; Table 4). Item-total correlations were low; the only correlations above $r=0.4$ were between "difficulty going to bed" and "difficulty falling asleep" $\left(r_{\mathrm{s}}=0.499\right)$. Because all age groups were together in one model, differences in frequency of sleep problems between groups may decrease correlations and consistency. 


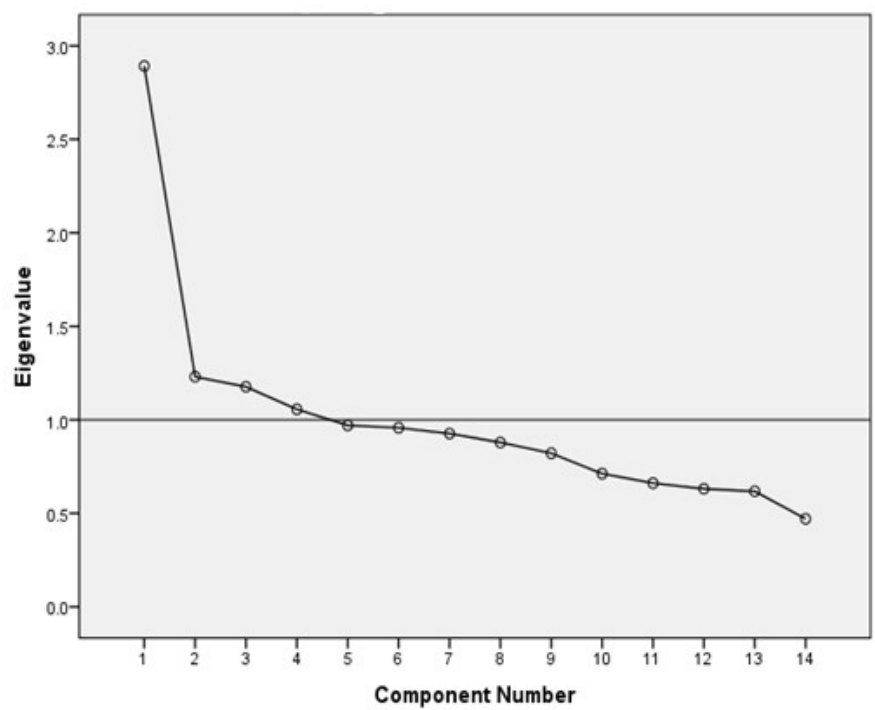

Figure 5: Model 1 Scree Plot

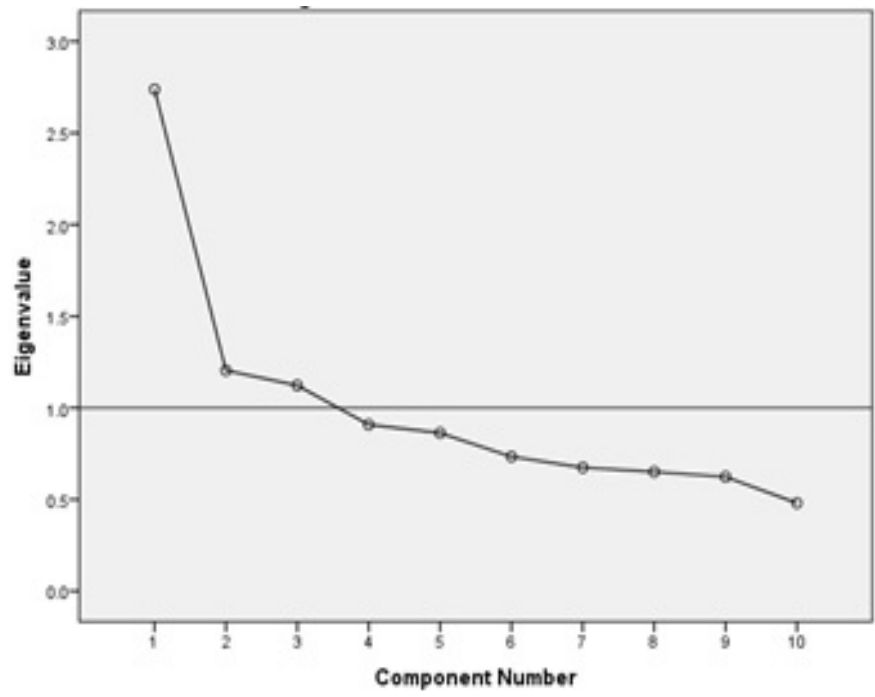

Figure 6: Model 2 Scree Plot 
Table 4: Factor Loadings of Principle Components Factor Analysis

\begin{tabular}{|c|c|c|c|c|c|c|}
\hline & \multicolumn{3}{|c|}{ Component (unrotated) matrix } & \multicolumn{3}{|c|}{ Pattern (rotated) matrix } \\
\hline & $\begin{array}{l}\text { Nighttime } \\
\text { problems }\end{array}$ & $\begin{array}{l}\text { Daytime } \\
\text { problems }\end{array}$ & $\begin{array}{c}\text { Sleep } \\
\text { duration } \\
\text { problems }\end{array}$ & $\begin{array}{l}\text { Nighttime } \\
\text { problems }\end{array}$ & $\begin{array}{l}\text { Daytime } \\
\text { problems }\end{array}$ & $\begin{array}{c}\text { Sleep } \\
\text { duration } \\
\text { problems }\end{array}$ \\
\hline $\begin{array}{l}\text { Difficulty going to } \\
\text { bed }\end{array}$ & 0.664 & & & 0.777 & & \\
\hline $\begin{array}{l}\text { Need to lay down } \\
\text { with child }\end{array}$ & 0.534 & & & 0.679 & & \\
\hline $\begin{array}{l}\text { Difficulty going to } \\
\text { sleep }\end{array}$ & 0.689 & & & 0.767 & & \\
\hline $\begin{array}{l}\text { Frequent/prolonged } \\
\text { awakenings }\end{array}$ & 0.486 & & & 0.482 & & \\
\hline $\begin{array}{l}\text { Difficulty waking } \\
\text { up in the morning }\end{array}$ & 0.559 & & & & 0.491 & \\
\hline Long/frequent naps & & 0.623 & & & 0.719 & \\
\hline $\begin{array}{l}\text { Regular duration is } \\
\text { not enough }\end{array}$ & 0.562 & & & & 0.436 & \\
\hline Daytime sleepiness & & 0.575 & & & 0.787 & \\
\hline $\begin{array}{l}\text { Irregular sleep } \\
\text { duration }\end{array}$ & & & 0.761 & & & 0.841 \\
\hline $\begin{array}{l}\text { Irregular bedtime } \\
\text { and wake time }\end{array}$ & & & 0.622 & & & 0.723 \\
\hline
\end{tabular}

\section{Cronbach's $\alpha$}

\begin{tabular}{lccc} 
Full sample & 0.6443 & 0.548 & 0.421 \\
Prepubertal & 0.649 & 0.518 & 0.454 \\
Pubertal & 0.650 & 0.572 & 0.456 \\
Post-pubertal & 0.576 & 0.610 & 0.177 \\
\hline
\end{tabular}




\subsubsection{Subgroup analysis}

In the pre-pubertal age group (4- to 8-years), 1,533 participants met eligibility requirements and were examined. Items loaded on the same factors as the full sample, with all items having a loading $>0.40$. The strongest factor loadings in this age group were "irregular sleep duration” (0.837) and "difficulty falling asleep” (0.803). Full-scale reliability was sufficient ( $\alpha=0.682$; Table 4$)$, and the removal of any one item did not impact the reliability.

The pubertal group (9- to 13-years) had 883 eligible participants who were evaluated. Inter-item correlations were all significant. One item, “frequent/prolonged awakenings,” did not have a favorable factor loading above 0.4 (0.317). The strongest factor loadings were on "daytime sleepiness” $(0.812)$ and “irregular sleep duration” (0.800), two elements expected to change during puberty (Crowley et al., 2014). Cronbach's $\alpha$ was acceptable $(\alpha=0.724)$, and would not increase if any items were deleted.

In the post-pubertal group (14- to 18-years), 321 participants were eligible and were examined. Individual measures of sampling adequacy were more problematic, dipping down to 0.563 ; and the KMO (0.672) was just above the suggested threshold of 0.60. Many of the correlations between the ten items were not significant, particularly with "need to lay down with child" and "frequent/prolonged naps." Factor analysis was attempted with these limitations in mind. PCA yielded four factors with eigenvalues greater than one. The fourth factor, although it was just above 1 (1.004) accounted for $10 \%$ of variance, making one wary to force it into another factor for the sake of a 3-factor full scale. "Irregular bedtime or wake time” loaded alone on the fourth factor with a strong loading (0.898). On the pattern matrix, "need to lay down with child" cross-loaded between factors 2 and 3 ("nighttime problems" and "sleep duration problems"), and "frequent/prolonged awakenings" did not achieve a unique loading above 0.4. The structure matrix better defined "need to lay down with child" as a member of factor 2 ("nighttime problems"), and raised factor loadings above 0.4 for "frequent/prolonged awakenings," which then proceeded to cross-load on factors 2 and 4 ("nighttime problems" and the factor as yet undefined). When 
three factors were forced, 'irregular bedtime or wake time’ had a communality as low as 0.103 , and did not have a factor loading greater than 0.4 .

\section{$3.5 \quad$ DISCUSSION}

The results obtained from evaluating the SSCSI indicate three main concepts underlying sleep problems in children with ASD: night time problems, day time problems, and problems with sleep duration. Sixteen dichotomous items taken from the raw questionnaire were reduced through principle components analysis and reliability testing to ten items in three factors: nighttime problems, daytime problems, and sleep duration problems. The ten items are in addition to the continuous variables, age and regular sleep duration, yielding a twelve-item questionnaire. Items that were removed from the model, such as "sleep disordered breathing," "wet the bed," and "sleepwalking/nightmares” may not be as much a problem in this sample as some other sleep problems. The reliability of the SSCSI was modest $(\alpha=0.694)$ indicating that additional refinement, perhaps in another ASD sample, is needed to further elucidate the concepts underlying sleep problems in children with ASD.

The post-pubertal group was not well-characterized by the SSCSI, with low communalities and factor loadings. Evaluating this group with the instrument, however, may give important information about how sleep patterns and problems change from childhood through adolescence. For instance, some sleep habits or problems, such as needing to lay down with the child or frequent/prolonged naps, would developmentally be expected to resolve. In addition, it is well-documented that the sleep period of postpubescent children shifts back to a later bedtime and wake time (Crowley et al., 2014; Tarokh \& Carskadon, 2010), which may help to explain why "difficulty waking in the morning” and "daytime sleepiness” load well in the postpubertal model. One could presume that perhaps sleep duration has stabilized after puberty so that "irregular bedtime and wake time” may not be a problem according to the 
SSCSI. Thought must be given to use of the instrument in this age group, and additional study may be needed to clarify these issues.

Although no other instruments were administered in the SSC sample to allow for analysis of convergent validity, that many items on the SSCSI are represented in other well-validated questionnaires

adds a measure of confidence in the SSCSI. Factor 1 of the SSCSI, nighttime problems, parallels the bedtime resistance, sleep onset delay, and night-waking subscales on the CSHQ and MSPSQ. The latter two questionnaires have parasomnia and sleep disordered breathing subscales, though these items were not retained in the SSCSI. Factor 2 of the SSCSI, daytime problems, resembles the daytime sleepiness scales in the CSHQ and MSPSQ, although the MSQSQ does not address difficulty waking and neither questionnaires has a "regular sleep duration not enough"-type item in their daytime scale. Finally, factor 3 of the SSCSI, sleep duration problems, corresponds to the sleep duration subscale of the CSHQ.

\subsubsection{Limitations}

The primary limitation of this study was unequal sizes between prepubertal, pubertal, and postpubertal groups. This may skew our ability to detect a similar factor structure in the SSC sample, and in the three groups. There was a slightly greater ratio of boys to girls (5.9:1) than that seen in the general ASD population (4.5:1). Another limitation was that little was known about the origin and administration of the SSCSI, and no other sleep instruments were used precluding the calculation of test-retest reliability, interrater reliability, and convergent validity. Without an objective measure of sleep, all items were based on parents' report, and subject to reporting bias. In addition, laying down with a child may be accepted in some cultures and families practicing co-bedding, and this was not taken into consideration. Finally, without also testing a control group of typically developing children, it was difficult to know what sleep problems are unique to ASD and which are common in all children of a certain age. 


\subsubsection{Implications}

Several implications may be drawn from this analysis. First, myriad previous reports of sleep problems in children with ASD were confirmed using the SSC sample. This indicates the need for clinical screening by health care professionals of sleep habits and patterns in their ASD patient population, and as suggested by Malow et al. (2014), parent education in proper sleep habits should be provided. Nurses are in an optimal position to use the SSCSI for screening both in-patient and out-patient populations. Although sleep is often disturbed in the hospital, nurses working with in-patients may observe abnormal sleep patterns in their patients, and, guided by the SSCSI, bring these to the attention of the healthcare team. As well as in primary care and sleep medicine clinics, screening with the SSCSI could be employed in a range of specialty clinics—neurology, pulmonology, cardiology, behavioral medicine, etc. This screening may aid in proper diagnosis and treatment of other symptoms, and contribute to holistic, multispecialty care. Pediatric homecare nurses have an advantage in screening for sleep problems because they may have opportunities to witness first hand bedtime problems, abnormal sleep patterns, and sequela such as difficulty waking in the morning and excess daytime sleepiness. In addition, homecare nurses often have time that other nurses may not to counsel families about improving sleep habits, and to evaluate treatment interventions.

Second, the SSCSI may be used by nurses and others utilizing the SSC sample for ASD research. As sleep problems in children with ASD have been correlated with gastrointestinal disorders, seizures (Aldinger et al., 2015), genes related to circadian rhythms (Melke et al., 2008; Yang et al., 2016), melatonin levels (Melke et al., 2008; Tordjman et al., 2005), cognitive performance (Elia et al., 2000; Limoges, Bolduc, Berthiaume, Mottron, \& Godbout, 2013), behavior problems (Schwichtenberg et al., 2013; Sikora, Johnson, Clemons, \& Katz, 2012), and developmental regression (Flavia Giannotti et al., 2011), the SSCSI may be used to evaluate sleep problems and patient outcomes in relation to many other variables in this large, well-characterized sample of children with ASD. 
Use of a validated instrument adds a measure of confidence to a study, and is essential for collection and interpretation of accurate patient data. It is important to state that this study is the first to explore the psychometrics of the SSCSI, and due to the data available, a full description of the reliability and validity was not possible. Before the SSCSI is used in full-scale studies, it must be tested in other samples of children and adolescents with ASD in order to establish test-retest reliability, inter-rater reliability, and to support our findings of internal consistency. The SSCSI must also be used along with validated sleep questionnaires such as the CSHQ, MSPSQ, and CGI-S in order to establish convergent validity.

\subsubsection{Conclusion}

In conclusion, the SSCSI was found to have three factors with 10 dichotomous and 2 continuous items that describe sleep problems in a large sample of children with ASD, and has adequate reliability and validity. Relatively low internal consistencies and crossloading of several items point to the need for further refinement of the instrument. Similar factor loading of items in three age groups, indicates that many sleep problems continue from childhood through adolescence; and similarity to factors in other well-validated sleep instruments adds confidence in the SSCSI. This study adds to the current knowledge base of sleep patterns in children with ASD, and reinforces the need for nurses and other healthcare providers to screen for and address possible sleep problems in their practice. The SSCSI also presents a means for other researchers using SSC data to integrate sleep pattern variables into their research, but must be further validated. 


\title{
4.0 DATA-BASED MANUSCRIPT: CHARACTERISTICS OF SLEEP IN CHILDREN WITH AUTISM SPECTRUM DISORDER FROM THE SIMONS SIMPLEX COLLECTION
}

\begin{abstract}
4.1 ABSTRACT
Background: About $40-80 \%$ of children with ASD have sleep problems, including bedtime behavior problems, difficulty falling and staying asleep, decreased sleep time, and daytime sleepiness. This study aimed to examine relationships between sleep problems and characteristics of children with ASD in a large, nationwide sample.

Methods: This secondary analysis of children 4- to 18-years explored sleep problems using the Simons Simplex Collection Sleep Interview (SSCSI), which includes Nighttime problems, Daytime problems, and Sleep duration problems subscales. Chi-square, Kruskal-Wallis, and Mann-Whitney U tests were performed to detect differences between age groups, sexes, and sleep problem severity groups. Odds ratios for variables associated with sleep problems were calculated using logistic regression. Two-way interaction effects were included in regression models, and stratified analyses performed for age groups. Results: Approximately 41\% of children had SSCSI sleep problems. The most common items were in the nighttime problems subscale; difficulty falling asleep was most frequently reported. More than onequarter slept less than the hours recommended for their age. The odds of sleep problems were highest for gastrointestinal distress (GID) and age. Low nonverbal IQ (NVIQ) and seizures increased the odds of composite and Nighttime problems. Males were more likely to have composite and Daytime problems. ASD severity was not associated with sleep problems in this sample.
\end{abstract}


Conclusions: This study advances our understanding of sleep in ASD by showing that GID, NVIQ, age and seizures increased the odds of sleep problems in children with ASD. These results reinforce the need to screen for sleep problems in children with ASD, and suggest future lines of inquiry.

\subsection{INTRODUCTION}

\subsubsection{Autism spectrum disorder}

Autism spectrum disorder (ASD) is a pervasive neurodevelopmental condition characterized by persistent difficulties in communication and social interaction, and restricted, repetitive behaviors, interests and activities. ASD is referred to as a “spectrum” because impairments may range from mild to severe, and may also include intellectual disability (American Psychiatric Association, 2013). The Autism and Developmental Disabilities Monitoring Network estimated that, in 2012, ASD affected 1 in 68 American children, and had a male-to-female ratio of 4.5:1 (Centers for Disease Control and Prevention, 2016).

\subsubsection{Sleep patterns in children with ASD}

An estimated $40-80 \%$ of children with ASD have comorbid sleep problems (Reviewed in Veatch, Maxwell-Horn, \& Malow, 2015; Cortesi, Giannotti, Ivanenko, \& Johnson, 2010), which have been recognized by researchers for decades (Ornitz et al., 1969; Tanguay et al., 1976). Studies have observed that, compared with typically developing [TD] peers, children with ASD have significantly longer sleep onset latency (time to fall asleep; Giannotti, Cortesi, Cerquiglini, Vagnoni, \& Valente, 2011, N=52; Goldman et al., 2017, N=41; Lambert et al., 2016, N=34), more frequent awakenings after sleep onset (Allik, Larsson, \& Smedje, 2008, N=32; Souders et al., 2009, N=99), shorter total sleep time (sleep beginning to end- awakenings after sleep onset; Elrod \& Hood, 2015, meta-analysis N=564; Goodlin- 
Jones, Tang, Liu, \& Anders, 2008, N=194; Miano et al., 2007, N=62), and poorer sleep efficiency (total sleep time/time in bed; all $p<0.05$; Elrod \& Hood, 2015; Fletcher et al., 2016, N=50; Giannotti et al., 2011).

It has been suggested that intellectual disability (ID; IQ < 70 with functional deficits; World Health Organisation, 1996) may alter sleep patterns in children with ASD. Two recent studies have noted that ASD with ID was associated with sleep anxiety in children 2- to 5-years-old ( $p<0.001)$, bedtime resistance and night wakings in children 6- to 18-years-old ( $p<0.05$; Mazurek \& Petroski, 2015), and short sleep duration in children 4- to 18-years-old ( $p<0.001$; Veatch et al., 2017). A meta-analysis of ten studies revealed that children with ASD and comorbid ID had significantly lower total sleep time than TD children (pooled difference of means $=-45$ minutes; 95\% CI: -62.6 to $-28.6, p<0.001$ ), while there was no significant difference in total sleep time between children with ASD and no ID compared to TD children $(p=0.316)$.

Severity of ASD may also contribute to abnormal sleep patterns in children. In a population of 1,224 children, those with parent-reported sleep problems (falling asleep and staying asleep) were observed to have higher ASD severity scores on the Autism Diagnostic Inventory-Revised social interaction, communication, and restricted, repetitive behavior subscales (Lord, Rutter, \& Le Couteur, 1994); and on the Vineland Adaptive Behavior Scale (Sparrow, Balla, Cicchetti, Harrison, \& Doll, 1984) communication, socialization, daily living skills, and motor skills subscales than children without sleep problems (Zachor \& Ben-Itzchak, 2016; $p<0.02$ ). Using the Autism Diagnostic Observation Scale (ADOS; Hus, Gotham, \& Lord, 2014; Lord et al., 1989) as an indicator of ASD severity, Veatch et al. (2017) observed that short sleep duration was significantly negatively correlated with ADOS social affect severity scores $(\rho=-0.06, p<0.02)$, though not with total ADOS severity score.

Two medical comorbidities that have been associated with sleep problems in children with ASD are gastrointestinal disturbance (GID) and seizures. Presence of epilepsy and epileptiform EEG abnormalities have both been reported as predictors of sleep problems (Giannotti et al., 2008; $p<0.01$ ). Aldinger, Lane, Veenstra-VanderWeele, and Levitt (2015) report that, among children with ASD and 
sleep problems (defined as parent report yes/no any sleep problems) in the Simons Simplex Collection (SSC), the odds of having GID (23\% of sample) were almost two times higher than for children without sleep problems (OR=1.8, 95\% CI: 1.5-2.3), and the odds of having both GID and seizures (2.6\% of sample) was twice as high as children without sleep problems (OR=2.0, 95\% CI: 1.3-3.1). In the Autism Genetic Resource Exchange, Aldinger et al. also report that children with sleep problems, seizures, and GID had a two-standard deviation lower Vineland Adaptive Behavior Scale composite score than children with none of the three comorbidities.

To date, most studies examining sleep problems in children with ASD have had small sample sizes and restricted age ranges, or look only at a single or select few sleep problems. The aim of this study is to examine the relationship between the presence of sleep problems and characteristics of children with ASD, such as age, sex, nonverbal IQ (NVIQ), ASD severity, and medical conditions in a large, nationwide sample of children 4- to 18-years-old. We hypothesize that the number of children with bedtime and nighttime sleep problems will decrease as children age; that children with GID and/or seizures will have more sleep problems; and that children with severe ASD and/or NVIQ will have more sleep problems.

\subsection{METHODS}

\subsubsection{Participants}

\subsubsection{Simons Simplex Collection}

The Simons Foundation Autism Research Initiative began in 2003 with the goal of improving the scientific understanding of ASD. One of their major projects was the Simons Simplex Collection [SSC], a sample of approximately 2,750 simplex families (only one child with ASD) from 12 sites in the United States and Canada. Two studies have examined sleep patterns in children from the SSC. Veatch et al. 
(2017) examined short sleep duration in relation to intellectual and behavioral comorbidities of ASD, and Aldinger et al. (2015) studied risk for fourteen medical conditions, including presence of any sleep problem (yes/no), in children with ASD. The present study will examine ten sleep problems, including short sleep duration, and how these relate to demographic, medical, and behavioral characteristics.

Procedures of the parent study, inclusion and exclusion criteria, and characteristics of the SSC study sample have been described previously (Fischbach \& Lord, 2010; Frazier et al., 2014; Lord et al., 2012). Briefly, children, aged 4- to 18 -years-old ( $\mathrm{N}=2,745, M=9.0, S D=0.07$ years), were strictly phenotyped through parent interview, medical history, and multiple well-validated instruments. Children were given a clinical best estimate diagnosis of ASD based on observation, medical history and chart review, and Autism Diagnostic Observation Schedule [ADOS] and Autism Diagnostic Interview-Revised [ADI-R] scores. Diagnoses were based on the Diagnostic and Statistical Manual of Mental Disorders-IVText Revised [DSM-IV-TR] (Simons Foundation Autism Research Initiative, 2014).

\subsubsection{Procedure}

This study was a secondary analysis of existing data from the Simons Foundation. For the parent study, informed consent was obtained from all parents or caregivers included in the study, and assent from children as required by local institutional review boards. Approval was obtained from the University of Pittsburgh Institutional Review Board for the present study. Data were de-identified by the Simons Foundation prior to receipt by the present researchers.

Demographic data (age, sex, etc.) were collected at the time of initiation in the parent study. In the current study, an attempt was made to capture normal shifts in sleep patterns throughout childhood by exploring the association of age with sleep patterns. Therefore, children in the SSC sample were divided by age into three age groups called early (4-8-years), middle (9-13- years), and late (14-18-years) childhood. Presence or absence of GID and seizures was determined using data from the medical history interview conducted by the parent study at baseline. 


\subsubsection{Measures}

Simons Simplex Collection Sleep Interview (SSCSI), an 11-item parent-report questionnaire, was used for assessing bedtime and nighttime sleep problems, daytime function, and sleep duration (Table 10). Questions were asked at a clinic visit or by telephone interview of parents within three months of study initiation. The scoring rubric consists of individual items that are scored as 0 for "no" and 1 for "yes". A continuous composite score is made by summing 10 dichotomous items, and adding 1 point for sleep duration that was outside the National Sleep Foundation recommendations for the child's age (Hirshkowitz et al., 2015). Composite scores range from 0 to 11, with higher scores indicating worse sleep. Scores for the nighttime problems and daytime problems subscales range from 0 to 4 , and scores for the sleep duration problems subscale range from 0 to 2. (reviewed in Johansson, Feeley, Chasens, in press). Participants were excluded from the present study if more than two items were missing or coded as unknown.

Autism Diagnostic Observation Schedule (ADOS) was used to measure ASD severity. The ADOS is a semi-structured observational instrument used with other instruments for diagnosis of ASD and classification of severity. The measures that make up the ADOS total score are operationalized as characteristics of social responsiveness, communication, language delay, and spatial ability. Four modules are available with developmentally appropriate tasks for testing individuals with different language abilities (Lord et al., 2000). Items are scored on a 4-point Likert-type scale from 0 (no abnormality) to 3 (moderate to severe abnormality), and fall into measures of either social affect or restricted repetitive behavior. Calibrated severity scores standardized for age and language level are calculated for social affect and restricted, repetitive behavior items, and a total ADOS severity score is the sum of these scores. Children with a total ADOS severity score from four to five are classified as having “autism spectrum,” and those with a score of six or more are classified as having "autism” (Gotham, Pickles, \& Lord, 2009; Gotham et al., 2008; Hus, Gotham, \& Lord, 2014). Standardization of module 4 scores to match prior standardization of modules 1-3 was performed by the present research team by 
mapping raw social affect and restricted, repetitive behavior scores onto total, social affect, and restricted repetitive behavior severity scores (V. Hus Bal, personal communication, March 24, 2017).

Four norm-referenced instruments were used by the parent study to quantify IQ in the SSC sample depending on chronologic and mental age: the Differential Ability Scales-II (DAS-II; Elliott, 2012; PsychCorp, 2016), Weschler Intelligence Scale for Children-Fourth Edition (WISC-IV; Weschler, 2003), Wechsler Abbreviated Scale of Intelligence (WASI; Wechsler, 2011), and Mullen Scales of Early Learning (MSEL; Bradley-Johnson, 1997). Scales have been standardized so that each scale produces a common verbal IQ (VIQ), nonverbal IQ (NVIQ), and full-scale IQ (FSIQ) score that can be used to quantify and compare IQ across instruments. The WISC-IV (Khor et al., 2014; Oliveras-Rentas et al., 2013; P. E. Williams et al., 2003) and MSEL (Akshoomoff, 2006; Bishop, Guthrie, Coffing, \& Lord, 2011) have been previously used in ASD samples. Discrepencies also often occur between nonverbal and verbal IQ scores in individuals with ASD, and tests that rely on language skills may not accurately reflect the child's actual intelligence and functional abilities. Thus, NVIQ scores will be utilized in the present study. Children were excluded from the present study if they were missing NVIQ and ADOS data.

Children were classified as having GID if they were reported (yes/no) to have bloating/excess gas, celiac disease, constipation, diarrhea, ulcers, gastroesophageal reflux disease, inflammatory bowel disease (Crohn's disease, ulcerative colitis), irritable bowel syndrome, abdominal pain, unusual stools, vomiting, and/or other GID; and classified as having seizures if they were reported to have atonic drop, infantile spasms; or partial complex, grand mal, petit mal, simple partial, and/or other seizures. GID and seizures were dichotomized as 0 (none reported) or 1 (one or more reported).

\subsubsection{Analysis}

Phenotypic data was acquired from the Simons Foundation software platform, SFARI Base. The ten

dichotomous SSCSI items, SSCSI composite score and subscale scores, NVIQ, ADOS severity score, and sex were described using frequency counts and percentages. Regular sleep duration in hours per night and 
age in years were described using mean, standard deviation, and range. Outliers in regular sleep duration-reported sleep duration less than 3 standard deviations below the population mean-were replaced with the $3^{\text {rd }}$ standard deviation below the mean (4.85 hours). Differences in NVIQ, ADOS severity score between age groups, sexes, and sleep problem groups (none/minimal, mild, moderate/severe) were explored with Mann-Whitney U or Kruskal-Wallis analyses; and differences in presence of GID and seizures between these groups were explored with chi-square analyses. Descriptive analyses were performed with SPSS version 23 (SPSS Inc., Chicago, IL).

SSCSI composite sleep scores have been categorized into three levels of sleep problems: none/minimal (scores 0-1), mild (2-3), moderate/severe (> 4). Baseline and adjacent category logistic regression analyses were performed to explore and compare the association between SSCSI composite and factor scores, and child characteristics. This method uses maximum likelihood estimation to compare the log odds of being in one group versus another. Six independent child characteristics variables were included: age, ADOS severity score (range: 4-10), sex (male=0, female=1), NVIQ $(<40=1,40-54=2$, 55$70=3,71-100=4,>100=5$ ), GID (no=0, yes=1), and seizures (no=0, yes=1). Nonverbal IQs above 70 were split into 2 groups: those above and those below or equal to the population mean of 100 . Those 70 and below were split into 3 groups using the population standard deviation $(\mathrm{SD}=15)$. The groups were 0 to 1 SD below 70 (NVIQ = 55-70), 1 to 2 SD (NVIQ=40-54), and >2 SD (NVIQ < 40). Based on recommendations from the American Association of Intellectual and Developmental Disabilities (Tasse, Luckasson, \& Nygren, 2013), NVIQ scores below 40 were condensed into one category. Where total ADOS severity score was significant, social affect and restricted, repetitive behavior severity scores were analyzed. Analyses were performed by building models with no/minimal sleep problems versus sleep problems (mild and moderate/severe) as the outcome variable, and then looking between sleep problem severities (none/minimal vs. mild, none/minimal vs. moderate/severe, mild vs. moderate/severe). The most parsimonious model in each case was chosen using the Akaike information criterion (AIC). Because of small proportions of children in the female, middle and late childhood, or seizure groups, only baseline (none/minimal sleep problems vs. sleep problems) analyses were performed in subgroups. Logistic 
regression analyses were done with the full sample. Two-way interactions between child characteristics were requested; and significant interactions between age or sex and the other child characteristics were reported. When there were interactions with age or sex, the sample was stratified by the significant variable (age or sex) and logistic regression analyses were run in the subgroups. Logistic regression was performed with the MASS package in the R suite (R Core Team, 2016). Level of significance was set at $p<0.05$.

\subsection{RESULTS}

\subsubsection{Sample characteristics}

Of the full sample (N=2,745), 2,619 children had sufficient data on the SSCSI, NVIQ, and ADOS measures to be used for the analysis (Table 5; see Table 13 for sample characteristics by SSCSI sleep score severity). One hundred twenty-six children were excluded from analysis: 22 because of missing more than 2 SSCSI items, and 104 because of not having SSCSI, NVIQ, and ADOS data. There were no significant differences between those included and excluded. All children had GID and seizure data. Among the variables used in this study, the SSCSI item, irregular bedtime/waketime, was the only variable missing in more than $5 \%$ of participants (missing $n=449,17.1 \%$ ). Children missing this item were significantly older (mean=9.5(3.4) vs. 8.9(3.6) years, $p<0.01$ ), and had significantly higher NVIQ (mean=90.2(25.1) vs. 83.3(26.2), $p<0.01)$.

Mean age at study inclusion was approximately 9-years-old (range: 4-18-years-old). Overall, there was a male-to-female ratio of 6.5:1. Half of all children were reported to have GID, a rate that was not significantly different between age groups. Significantly more children with moderate/severe sleep problems had GID than those with mild or none/minimal sleep problems; and significantly more children with mild sleep problems have GID than those with none/minimal sleep problems (moderate/severe: 
60.9\%, mild: $52.5 \%$, and none/minimal: $44.8 \% p<0.05)$. Approximately 5\% of children were reported to have a seizure condition. Significantly fewer children in early childhood had seizures than in middle and late childhood (early: 4.2\%, middle: $6.1 \%$, late: $7.3 \%$ respectively, $p<0.05$ ); and significantly fewer children with none/minimal sleep problems had seizures than those with moderate/severe sleep problems (4.3\% vs. $7.1 \%, p<0.05)$.

In the current sample, over one-quarter (26.6\%) of children had a NVIQ $\leq 70$. The proportion of children with NVIQ $\leq 70$ was not different between age groups, but was significantly higher in girls than boys $(38.0 \%$ vs. $24.8 \%, p<0.001)$, in children diagnosed with autism than ASD (28.5\% vs. 9.9\%, $p<0.001)$, and in children with moderate/severe than none/minimal sleep problems $(31.2 \%$ vs. $24.8 \%$, $p<0.05$ ). Total ADOS severity score and number of children with ASD (ADOS=4-5) versus autism (ADOS=6-10) were not found to be significantly different between girls and boys $(p>0.05)$. Significantly more children with none/minimal vs. mild sleep problems had ASD (12.1\% vs. 9.2\%, $p<0.05)$. 
Table 5: SSC Sample Characteristics

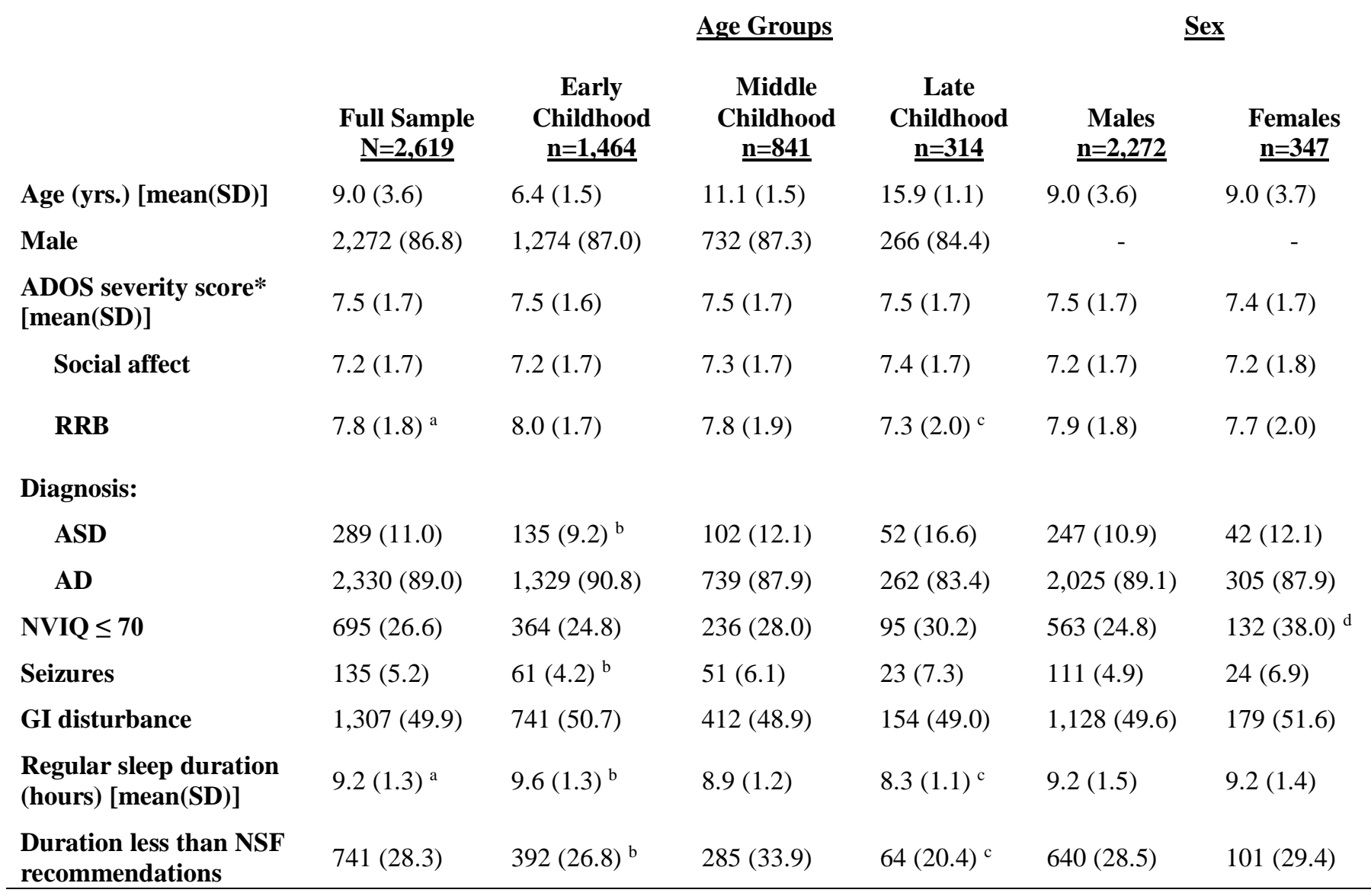

Note: data presented as n (\%) unless otherwise specified; *ADOS=Autism Diagnostic Observation Scale, range 4-10;

ASD=autism spectrum disorder (ADOS score 4-5); $\mathrm{AD}=$ autism disorder (ADOS score 6-10); GI= gastrointestinal;

NSF=National Sleep Foundation; NVIQ=nonverbal IQ; RRB=restricted and repetitive behavior ; Difference between early, middle, and late childhood, Kruskal-Wallis, $p<0.05$; ${ }^{\mathrm{b}}$ Difference between early and middle childhood, $p<0.05$; ${ }^{\mathrm{C}}$ Difference

between middle and late childhood, $p<0.05$; ${ }^{\mathrm{d}}$ Difference between males and females, $p<0.05$ 


\subsubsection{SSCSI items}

Table 6 displays the number and proportion of children whose parents responded "yes" to each SSCSI item, indicating that the item was problematic for the child (see Table 14 for distribution of SSCSI sleep score categories). In the total sample of 2,619 children, 1,623 (62\%) of parents responded “yes” to one or more SSCSI items. The most commonly reported SSCSI items were in the nighttime problems subscale, with up to $32 \%$ of parents reporting “yes” to at least one item. Similarly, parents reported that up to onesixth of children had at least one problem item on the SSCSI daytime problems subscale. Significantly more females than males were reported to have regular sleep duration that was not enough (26.4\% vs. $20.6 \%, p<0.02)$, difficulty waking in the morning (19.3\% vs. $12.5 \%, p<0.01)$, long/frequent naps $(5.8 \%$ vs. $3.5 \%, p<0.04)$, and excess daytime sleepiness $(17.1 \%$ vs. $12.9 \%, p<0.04)$. Few children were reported to have all items for nighttime problems (3.1\%), daytime problems $(0.7 \%)$, or sleep duration problems (2.7\%) subscales, and only one child had the maximum SSCSI composite score of 11 . Mean sleep duration was just over 9 hours (range: 4.9-13 hours), and steadily declined as children aged (correlation between age and sleep duration $r=-0.361, p<0.01)$. About one-quarter of children $(28.7 \%)$ slept less than the number of hours recommended for their age by the National Sleep Foundation (NSF; Hirshkowitz et al., 2015). The proportion of children with regular sleep duration less than that recommended by the NSF increased from early (27.8\%) to middle childhood, (33.9\%) and then decreased from middle to late childhood (20.1\%). 
Table 6: SSCSI Reported Item Frequencies

\begin{tabular}{|c|c|c|c|c|c|c|}
\hline & \multicolumn{4}{|c|}{ Age groups } & \multicolumn{2}{|c|}{$\underline{\text { Sex }}$} \\
\hline & $\begin{array}{l}\text { Total Sample } \\
\underline{\mathrm{N}=2,619}\end{array}$ & $\begin{array}{c}\text { Early } \\
\text { Childhood } \\
\underline{\mathbf{n}=1,464}\end{array}$ & $\begin{array}{c}\text { Middle } \\
\text { Childhood } \\
\underline{\mathbf{n}=841}\end{array}$ & $\begin{array}{c}\text { Late } \\
\text { Childhood } \\
\underline{\mathbf{n}=314}\end{array}$ & $\begin{array}{c}\text { Males } \\
\underline{\mathrm{n}=2,272}\end{array}$ & $\begin{array}{c}\text { Females } \\
\underline{\mathbf{n}=347}\end{array}$ \\
\hline \multicolumn{7}{|l|}{ Nighttime problems } \\
\hline Bedtime problems & $465(17.8)^{a}$ & $280(19.1)$ & $146(17.4)$ & $39(12.4)^{c}$ & 396 (17.4) & 69 (19.9) \\
\hline $\begin{array}{l}\text { Need to lay down with } \\
\text { child }\end{array}$ & $498(19.1)^{\mathrm{a}}$ & $363(24.9)^{b}$ & $121(14.5)$ & $14(4.5)^{c}$ & $433(19.1)$ & 65 (18.9) \\
\hline Difficulty falling asleep & $829(31.8)^{a}$ & $437(30.0)^{b}$ & $296(35.4)$ & 96 (30.6) & $706(31.2)$ & $123(35.7)$ \\
\hline $\begin{array}{l}\text { Frequent/prolonged } \\
\text { awakenings }\end{array}$ & $489(18.8)^{a}$ & $300(20.5)^{b}$ & $143(17.1)$ & $46(14.7)$ & $416(18.4)$ & $73(21.2)$ \\
\hline \multicolumn{7}{|l|}{ Daytime problems } \\
\hline Difficulty waking & $351(13.4)$ & $178(12.2)$ & $132(15.7)$ & $41(13.1)$ & $284(12.5)^{d}$ & 67 (19.3) \\
\hline Excess daytime sleepiness & $350(13.4)$ & $182(12.5)$ & $117(14.0)$ & $51(16.3)$ & $291(12.9)^{d}$ & $59(17.1)$ \\
\hline Long/ frequent naps & $99(3.8)$ & $56(3.8)$ & $26(3.1)$ & $17(5.4)$ & $79(3.5)^{d}$ & $20(5.8)$ \\
\hline $\begin{array}{l}\text { Regular duration is not } \\
\text { enough }\end{array}$ & $537(21.4)$ & $293(20.8)$ & $183(22.6)$ & $61(20.5)$ & $449(20.6)^{d}$ & $88(26.4)$ \\
\hline \multicolumn{7}{|l|}{ Sleep duration problems } \\
\hline $\begin{array}{l}\text { Irregular bedtime and/or } \\
\text { waketime * }\end{array}$ & 425 (19.6) & 235 (18.9) & $133(19.8)$ & $57(22.1)$ & 361 (19.2) & $64(22.2)$ \\
\hline No regular duration & $146(5.6)$ & $80(5.5)$ & $44(5.2)$ & $22(7.1)$ & $123(5.4)$ & $23(6.6)$ \\
\hline
\end{tabular}

All n (\%); * missing 17.1\%; ${ }^{\text {a }}$ Difference between early, middle, and late childhood, $p<0.05$; ${ }^{\mathrm{b}}$ Difference between early and middle childhood, $p<0.05 ;{ }^{\mathrm{c}}$ Difference between middle and late childhood, $p<0.05$; ${ }^{\mathrm{d}}$ Difference between males and females, $p<0.05$ 


\subsubsection{SSCSI composite and subscale scores}

Approximately $41 \%$ of children were categorized as having a mild (SSCSI composite score 2-3) or moderate/severe (SSCSI composite score $\geq 4$ ) sleep problem (Table 7; Table 15 for SSCSI composite and subscale frequencies by sleep score categories). The proportion of children with sleep problems was significantly less in the late childhood group (34.1\%) than the early and middle childhood groups (47.4\% vs. $46.3 \%$ respectively, $p<0.001$ ). The distribution of SSCSI composite and subscale scores were not different between those with ASD (ADOS CSS 4-5) versus autism (ADOS CSS $\geq 6$ ). Mean SSCSI composite score was significantly greater in children with NVIQ $\leq 70$ compared to those with NVIQ $>70$ (2.1(2.1) vs. 1.8(1.9), $p<0.01)$. In addition, children with NVIQ $<55$ had significantly more need for a parent to lay down with them (28.0\% vs. $18.2 \%, p<0.01)$, more frequent/prolonged awakenings $(29.2 \%$ vs. $22.1 \%, p<0.05$ ), and irregular bedtime/waketime than children with NVIQ 55-70 (30.0\% vs. 22.7\%, $p<0.05)$. 
Table 7: SSCSI Composite and Subscale Frequencies Among Children with Sleep Problems

\begin{tabular}{|c|c|c|c|c|c|c|}
\hline & \multicolumn{4}{|c|}{ Age groups } & \multicolumn{2}{|c|}{$\underline{\text { Sex }}$} \\
\hline & $\begin{array}{c}\text { Full Sample } \\
\underline{N=2,619}\end{array}$ & $\begin{array}{c}\text { Early } \\
\text { Childhood } \\
\underline{\mathbf{n}=1,464}\end{array}$ & $\begin{array}{c}\text { Middle } \\
\text { Childhood } \\
\underline{\mathbf{n}=\mathbf{8 4 1}}\end{array}$ & $\begin{array}{c}\begin{array}{c}\text { Late } \\
\text { Childhood } \\
\underline{\mathbf{n}=314}\end{array} \\
\end{array}$ & $\begin{array}{c}\begin{array}{c}\text { Males } \\
\mathbf{n}=2,272\end{array} \\
\end{array}$ & $\begin{array}{c}\begin{array}{c}\text { Females } \\
\mathbf{n}=347\end{array} \\
\end{array}$ \\
\hline Composite & $1,073(41.0)^{\mathrm{a}}$ & $635(43.4)^{d}$ & 331 (39.4) & 107 (34.1) & $900(39.6)^{e}$ & 173 (49.9) \\
\hline $\begin{array}{l}\text { Nighttime } \\
\text { problems }\end{array}$ & $675(25.8)^{\mathrm{a}}$ & $406(27.7)^{b, d}$ & 213 (25.3) & $56(17.8)$ & $577(25.4)$ & $98(28.2)$ \\
\hline $\begin{array}{l}\text { Daytime } \\
\text { problems }\end{array}$ & 348 (13.3) & $192(13.1)$ & 115 (13.7) & 41 (13.1) & $726(32.0)^{\mathrm{e}}$ & $147(42.4)$ \\
\hline $\begin{array}{l}\text { Sleep } \\
\text { duration } \\
\text { problems }\end{array}$ & $72(2.7)$ & $45(3.1)$ & $21(2.5)$ & $6(1.9)$ & $60(2.6)$ & $12(3.5)$ \\
\hline
\end{tabular}

Note: "Sleep problems" considered SSCSI score $\geq 2 ;{ }^{\text {a }}$ Difference between early, middle, and late childhood, $p<0.05$; ${ }^{\mathrm{b}}$ Difference between early and middle childhood, $p<0.05$; ${ }^{\mathrm{c}}$ Difference between middle and late childhood, $p<0.05$; ${ }^{\mathrm{d}}$ Difference between early and late childhood, $p<0.05$; ${ }^{\mathrm{e}}$ Difference between males and females, $p<0.05$

\subsubsection{Full sample associations with SSCSI sleep problems}

Odds ratios, 95\% confidence intervals, and p-values reflecting the association between child characteristics (age, sex, NVIQ, ADOS severity score, GID, and seizures) and SSCSI scores for the full sample can be found in Table 8. When examining the odds of a child having SSCSI composite sleep problems (mild-moderate/severe) as opposed to none/minimal sleep problems, GID was the strongest risk factor $(\mathrm{OR}=2.79,95 \% \mathrm{CI}$ : 1.59-4.92, $p<0.01)$, followed lower NVIQ (OR=0.73, 95\% CI: 0.59-0.90, $p<0.01)$ and younger age $(\mathrm{OR}=0.76,95 \% \mathrm{CI}: 0.66-0.87, p<0.01)$ controlling for sex and ADOS severity score. An interaction was present between age and NVIQ for composite vs. none/minimal sleep problems (OR=1.04, 95\% CI 1.02-1.06, $p<0.01$ ). Seizures had a significant, large OR, but an extremely wide 95\% CI (OR=7.38, 95\% CI: 1.34-43.18, $p=0.02)$, likely due to the small number of children with seizures. Of those who had sleep problems, the odds of having mild sleep problems (vs. none/minimal) was associated with GID (OR=2.63, 95\% CI: 1.29-5.40, $p=0.01)$, male sex (OR=1.66, 95\% CI: 1.26-2.18, $p<0.01)$, 
younger age (OR=0.87, 95\% CI: 0.79-0.96, $p<0.01)$, and higher ADOS severity score (OR=1.07, 95\% CI: 1.01-1.13, $p=0.03$ ) and ADOS social affect severity score (OR=1.09, 95\% CI: 1.03-1.15, $p<0.01)$. A significant interaction was present between age and NVIQ for mild vs. none/minimal problems (OR=1.03, 95\% CI: 1.01-1.06, $p=0.02)$. The odds of moderate/severe sleep problems were significantly greater as NVIQ decreased (vs. none/minimal: $\mathrm{OR}=0.62,0.48-0.79$; or mild: $\mathrm{OR}=0.80,95 \%$ CI: 0.68 0.95 , all $p \leq 0.01$ ), as age increased (vs. none/minimal: $\mathrm{OR}=0.84,95 \% \mathrm{CI}: 0.75-0.92, p<0.01$ ), and with the presence of GID (OR=1.88, 95\% CI: 1.51-2.34, $p<0.01)$. The odds of moderate/severe composite sleep problems increased as ADOS total severity score and social affect severity score decreased (vs. mild; total ADOS: OR=0.91, 95\% CI: 0.84-0.98; social affect: OR=0.89, 95\% CI: 0.82-0.96, all $p \leq 0.02$ ). Restricted, repetitive behavior severity score was not significantly associated with the SSCSI composite sleep score. A significant interaction was found between age and NVIQ in those with moderate/severe composite sleep problems (OR=1.04, 95\% CI: 1.01-1.07, $p<0.01)$.

The odds of nighttime problems (mild-moderate/severe vs. none/minimal) were significantly greater in younger children (OR=0.85, 95\% CI: 0.78-0.93, $p<0.01)$, those with lower NVIQ (OR=0.70, 95\% CI: 0.57-0.86, $p<0.01$ ), and those with seizures (OR=1.47, 95\% CI: 1.01-2.14, $p=0.04)$. A significant interaction was present between age and NVIQ (OR=1.02, 95\% CI: 1.01-1.05, $p=0.03$ ). The odds of mild nighttime problems (vs. none/minimal problems) and moderate/severe nighttime problems (vs. none/minimal and mild problems) were significantly greater in younger children (mild vs. none/minimal: OR=0.89, 95\% CI: 0.81-0.96; moderate/severe vs. none/minimal: OR=0.81, 95\% CI: 0.69-0.96; moderate/severe vs. mild: OR=0.85, 95\% CI: $0.73-0.97$; all $p \leq 0.03$ ). Children with GID were significantly more likely to have mild (vs. none/minimal) nighttime problems (OR=2.68, 95\% CI: 1.146.34, $p=0.02$ ). Lower NVIQ also increased the likelihood of mild (vs. none/minimal) nighttime problems $(\mathrm{OR}=0.71,95 \% \mathrm{CI}: 0.57-0.88, p<0.01)$, and a significant interaction was found between age and NVIQ in children with mild (vs. none/minimal) nighttime problems (OR=1.02, 95\% CI: >1.00-1.05, p=0.04).

The odds of daytime problems (mild-moderate/severe vs. none/minimal) were more than three times greater for males and those with GID (male: OR=3.37, 95\% CI: 1.50-7.63; GID: OR=3.17, 95\% CI: 
1.72-5.88, all $p<0.01$ ). The odds of composite (vs. mild-moderate/severe problems) and mild (vs. none/minimal) daytime problems were significantly greater in younger children (composite: OR=0.90, 95\% CI: 0.83-0.98; mild vs. none/minimal: OR=0.88, 95\% CI: 0.79-0.996; all $p<0.05)$. However, the odds of moderate/severe problems were significantly greater in older children (vs. none/minimal: OR=1.80, 95\% CI: 1.08-2.93; vs. mild: OR=1.79, 95\% CI: 1.03-3.19; all $p<0.05$ ). A significant interaction was found for children with daytime problems between age and NVIQ (mild-moderate/severe vs. none/minimal: OR=1.03, 95\% CI: 1.01-1.05, $p<0.01$ ), and between age and ADOS (moderate/severe vs. none/minimal: OR=0.93, 95\% CI: 0.87-0.99, $p=0.03$ ). There were no associations between odds of sleep duration problems (mild-moderate/severe vs. none/minimal) and any child characteristics.

\subsubsection{Subgroup associations with SSCSI sleep problems}

Since there were numerous interactions between age and other covariates, the sample was stratified by age group (early, middle, and late childhood) and associations between sleep problems and child characteristics were further explored (Tables 18-20). Examining only the early childhood group (4-8years-old), low NVIQ was significantly associated with increased odds of composite sleep problems (OR=0.82, 95\% CI: 0.74-0.90; $p<0.01)$, nighttime problems (OR=0.80, 95\% CI: 0.72-0.89; $p<0.01$ ), and sleep duration problems (OR=0.65, 95\% CI: 0.52-0.82, $p<0.01)$. Presence of GID significantly increased the odds of composite sleep problems (OR=1.61, 95\% CI: 1.31-1.99, $p<0.01)$, nighttime problems (OR=1.37, 95\% CI: 1.09-1.73, $p<0.01)$ and daytime problems (OR=2.19, 95\% CI: 1.60-3.04, $p<0.01)$. Males in the early childhood group were significantly more likely to experience daytime problems than females (OR=1.59, 95\% CI: 1.05-2.37, $p=0.02)$.

Children in the middle childhood group (8-13-years-old) had significant associations between age and the odds of composite problems ( $\mathrm{OR}=1.15,95 \% \mathrm{CI}: 1.04-1.27, p<0.01)$ and daytime problems $(\mathrm{OR}=1.18,95 \% \mathrm{CI}: 1.03-1.36, p<0.01)$. Males were significantly more likely than females to have composite sleep problems $(\mathrm{OR}=1.86,95 \% \mathrm{CI}$ : 1.23-2.83, $p<0.01)$. Presence of GID significantly 
increased the odds of composite problems, nighttime problems, and daytime problems (composite: OR=1.56, 95\% CI: 1.18-2.06; nighttime problems: OR=1.63, 95\% CI: 1.19-2.24, $p<0.01)$. As NVIQ decreased, the odds of sleep duration problems significantly increased (OR=0.73, 95\% CI: 0.54-0.99, $p=0.04)$. Presence of GID also increased the odds of nighttime problems two-fold in the late-childhood group (OR=2.08, 95\% CI: 1.15-3.85, $p=0.02)$. 
Table 8: Associations Between Child Characteristics and SSCSI Sleep Problems

\begin{tabular}{|c|c|c|c|c|c|c|c|c|c|c|c|c|c|}
\hline & & \multicolumn{3}{|c|}{$\begin{array}{c}\text { None/minimal vs. } \\
\text { Mild-severe }\end{array}$} & \multicolumn{3}{|c|}{$\begin{array}{c}\text { None/minimal vs. } \\
\text { Mild }\end{array}$} & \multicolumn{3}{|c|}{$\begin{array}{c}\text { None/minimal vs. } \\
\text { Moderate/severe }\end{array}$} & \multicolumn{3}{|c|}{$\begin{array}{c}\text { Mild } \\
\text { vs. Moderate/severe }\end{array}$} \\
\hline & & OR & CI & $p$ & OR & CI & $p$ & OR & CI & $p$ & OR & CI & $p$ \\
\hline \multirow{8}{*}{$\begin{array}{l}\text { SSCSI } \\
\text { composite } \\
\text { sleep score }\end{array}$} & Age & 0.97 & $\begin{array}{l}0.95- \\
0.99\end{array}$ & $<0.01$ & 0.97 & $\begin{array}{l}0.95- \\
0.99\end{array}$ & 0.04 & 0.96 & $\begin{array}{l}0.93- \\
0.99\end{array}$ & $<0.01$ & 0.99 & $\begin{array}{l}0.96- \\
1.03 \\
\end{array}$ & 0.72 \\
\hline & Sex $*$ & 1.47 & $\begin{array}{l}1.17- \\
1.85\end{array}$ & $<0.01$ & 1.58 & $\begin{array}{l}1.22- \\
2.06\end{array}$ & $<0.01$ & 1.35 & $\begin{array}{l}0.98- \\
1.79\end{array}$ & 0.07 & 0.84 & $\begin{array}{l}0.61- \\
1.16\end{array}$ & 0.30 \\
\hline & NVIQ & 0.88 & $\begin{array}{l}0.81- \\
0.95\end{array}$ & 0.08 & 0.98 & $\begin{array}{l}0.90- \\
1.07\end{array}$ & 0.66 & 0.88 & $\begin{array}{l}0.81- \\
0.96\end{array}$ & $<0.01$ & 0.89 & $\begin{array}{l}0.81- \\
0.98\end{array}$ & 0.02 \\
\hline & ADOS & 1.02 & $\begin{array}{l}0.97- \\
1.07\end{array}$ & 0.40 & 1.05 & $\begin{array}{l}0.99- \\
1.12 \\
\end{array}$ & 0.05 & 0.97 & $\begin{array}{l}0.91- \\
1.04\end{array}$ & 0.40 & 0.92 & $\begin{array}{l}0.86- \\
0.99\end{array}$ & 0.02 \\
\hline & $\mathbf{S A}^{\mathrm{t}}$ & - & - & - & - & - & - & - & - & - & 0.93 & $\begin{array}{l}0.86- \\
0.99\end{array}$ & 0.03 \\
\hline & $\mathbf{R R B}^{\mathrm{t}}$ & - & - & - & - & - & - & - & - & - & 0.97 & $\begin{array}{l}0.91- \\
1.03\end{array}$ & 0.35 \\
\hline & GID & 1.53 & $\begin{array}{l}1.30- \\
1.78\end{array}$ & $<0.01$ & 1.34 & $\begin{array}{l}1.12- \\
1.61\end{array}$ & $<0.01$ & 1.84 & $\begin{array}{l}1.50- \\
2.28\end{array}$ & $<0.01$ & 1.38 & $\begin{array}{l}1.09- \\
1.75\end{array}$ & $<0.01$ \\
\hline & Seizures & 1.33 & $\begin{array}{l}0.93- \\
1.90 \\
\end{array}$ & 0.12 & 1.26 & $\begin{array}{l}0.82- \\
1.93 \\
\end{array}$ & 0.28 & 1.41 & $\begin{array}{l}0.90- \\
2.18 \\
\end{array}$ & 0.13 & 1.16 & $\begin{array}{l}0.72- \\
1.88 \\
\end{array}$ & 0.54 \\
\hline \multicolumn{14}{|l|}{ Subscales } \\
\hline \multirow{7}{*}{$\begin{array}{l}\text { Nighttime } \\
\text { problems }\end{array}$} & Age & 0.95 & $\begin{array}{l}0.93- \\
0.98\end{array}$ & $<0.01$ & 0.96 & $\begin{array}{l}0.94- \\
0.99\end{array}$ & $<0.01$ & 0.90 & $\begin{array}{l}0.84- \\
0.96\end{array}$ & $<0.01$ & 0.94 & $\begin{array}{l}0.87- \\
1.004\end{array}$ & 0.07 \\
\hline & Sex $*$ & 1.10 & $\begin{array}{l}0.85- \\
1.43 \\
\end{array}$ & 0.45 & 1.12 & $\begin{array}{l}0.86- \\
1.46 \\
\end{array}$ & 0.40 & 0.99 & $\begin{array}{l}0.51- \\
1.91 \\
\end{array}$ & 0.98 & 0.91 & $\begin{array}{l}0.46- \\
1.80\end{array}$ & 0.78 \\
\hline & NVIQ & 0.88 & $\begin{array}{l}0.81- \\
0.95\end{array}$ & $<0.01$ & 0.88 & $\begin{array}{l}0.81- \\
0.95\end{array}$ & $<0.01$ & 0.84 & $\begin{array}{l}0.70- \\
1.02\end{array}$ & 0.08 & 0.99 & $\begin{array}{l}0.82- \\
1.19\end{array}$ & 0.89 \\
\hline & ADOS & 0.99 & $\begin{array}{l}0.94- \\
1.05 \\
\end{array}$ & 0.73 & 0.99 & $\begin{array}{l}0.93- \\
1.04 \\
\end{array}$ & 0.66 & 1.01 & $\begin{array}{l}0.88- \\
1.16 \\
\end{array}$ & 0.86 & 1.03 & $\begin{array}{l}0.90- \\
1.20 \\
\end{array}$ & 0.60 \\
\hline & $\mathbf{S A}^{\mathrm{t}}$ & - & - & - & - & - & - & - & - & - & - & - & - \\
\hline & $\mathbf{R R B}^{\mathrm{t}}$ & - & - & - & - & - & - & - & - & - & - & - & - \\
\hline & GID & 1.49 & $\begin{array}{l}1.25- \\
1.78\end{array}$ & $<0.01$ & 1.43 & $\begin{array}{l}1.19- \\
1.73\end{array}$ & $<0.01$ & 1.94 & $\begin{array}{l}1.23- \\
3.12\end{array}$ & $<0.01$ & 1.39 & $\begin{array}{l}0.86- \\
2.26\end{array}$ & 0.18 \\
\hline
\end{tabular}




\begin{tabular}{|c|c|c|c|c|c|c|c|c|c|c|c|c|c|}
\hline & Seizures & 1.33 & $\begin{array}{l}0.91- \\
1.93\end{array}$ & 0.14 & 1.35 & $\begin{array}{l}0.91- \\
1.99\end{array}$ & 0.13 & 1.19 & $\begin{array}{l}0.46- \\
3.10\end{array}$ & 0.72 & 0.86 & $\begin{array}{l}0.32- \\
2.29\end{array}$ & 0.76 \\
\hline \multirow{8}{*}{$\begin{array}{c}\text { Daytime } \\
\text { problems }\end{array}$} & Age & 0.98 & $\begin{array}{l}0.79- \\
1.21 \\
\end{array}$ & 0.84 & 0.98 & $\begin{array}{l}0.79- \\
1.22 \\
\end{array}$ & 0.85 & 0.97 & $\begin{array}{l}0.40- \\
2.32 \\
\end{array}$ & 0.94 & 1.03 & $\begin{array}{l}0.90- \\
1.17 \\
\end{array}$ & 0.64 \\
\hline & Sex $*$ & 1.57 & $\begin{array}{l}1.15- \\
2.12 \\
\end{array}$ & $<0.01$ & 1.63 & $\begin{array}{l}1.19- \\
2.20 \\
\end{array}$ & $<0.01$ & 0.80 & $\begin{array}{l}0.18- \\
3.50\end{array}$ & 0.77 & 0.41 & $\begin{array}{l}0.09- \\
1.92 \\
\end{array}$ & 0.26 \\
\hline & NVIQ & 1.08 & $\begin{array}{l}0.98- \\
1.20\end{array}$ & 1.13 & 1.09 & $\begin{array}{l}0.98- \\
1.21\end{array}$ & 0.12 & 1.03 & $\begin{array}{l}0.70- \\
1.52\end{array}$ & 0.89 & 1.05 & $\begin{array}{l}0.70- \\
1.56\end{array}$ & 0.83 \\
\hline & ADOS & 1.00 & $\begin{array}{l}0.93- \\
1.07 \\
\end{array}$ & 0.94 & 1.00 & $\begin{array}{l}0.93- \\
1.07 \\
\end{array}$ & 0.97 & 0.93 & $\begin{array}{l}0.71- \\
1.21 \\
\end{array}$ & 0.59 & 1.11 & $\begin{array}{l}0.81- \\
1.50 \\
\end{array}$ & 0.52 \\
\hline & $\mathbf{S A}^{\mathrm{t}}$ & - & - & - & - & - & - & - & - & - & - & - & - \\
\hline & $\mathbf{R R B}^{\mathrm{t}}$ & - & - & - & - & - & - & - & - & - & - & - & - \\
\hline & GID & 1.98 & $\begin{array}{l}1.57- \\
2.51 \\
\end{array}$ & $<0.01$ & 1.98 & $\begin{array}{l}1.56- \\
2.53 \\
\end{array}$ & $<0.01$ & 1.90 & $\begin{array}{l}0.75- \\
4.85 \\
\end{array}$ & 0.18 & 0.83 & $\begin{array}{l}0.31- \\
2.24 \\
\end{array}$ & 0.71 \\
\hline & Seizures & 1.16 & $\begin{array}{l}0.73- \\
1.86 \\
\end{array}$ & 0.53 & 1.00 & $\begin{array}{l}0.60- \\
1.67 \\
\end{array}$ & 0.99 & 5.13 & $\begin{array}{l}1.45- \\
14.41\end{array}$ & $<0.01$ & 4.35 & $\begin{array}{l}1.16- \\
13.42\end{array}$ & 0.02 \\
\hline \multirow{8}{*}{$\begin{array}{l}\text { Sleep } \\
\text { duration } \\
\text { problems }\end{array}$} & Age & 0.95 & $\begin{array}{l}0.88- \\
1.02 \\
\end{array}$ & 0.14 & \multirow{8}{*}{\multicolumn{9}{|c|}{$\begin{array}{l}\text { Bold results denote statistical significance; Note: Sleep duration problems sample too } \\
\text { small to examine differences between none vs. mild, none vs. moderate/severe, or mild } \\
\text { vs. moderate/severe; * Reference group=females; }{ }^{\mathrm{t}} \text { only tested if ADOS severity } \\
\text { score significant; ADOS=ADOS severity score; CI=95\% confidence interval; } \\
\text { GID=gastrointestinal disturbance; NVIQ=non-verbal IQ; OR=odds ratio; SA=ADOS } \\
\text { social affect severity score; RRB=ADOS restricted, repetitive behavior severity score }\end{array}$}} \\
\hline & Sex $*$ & 1.32 & $\begin{array}{l}0.70- \\
2.48\end{array}$ & 0.39 & & & & & & & & & \\
\hline & NVIQ & 0.69 & $\begin{array}{l}0.58- \\
0.82\end{array}$ & $<0.01$ & & & & & & & & & \\
\hline & ADOS & 1.00 & $\begin{array}{l}0.87- \\
1.15\end{array}$ & 0.97 & & & & & & & & & \\
\hline & $\mathbf{S A}^{\mathrm{t}}$ & - & - & - & & & & & & & & & \\
\hline & $\mathbf{R R B}^{\mathrm{t}}$ & - & - & - & & & & & & & & & \\
\hline & GID & 1.11 & $\begin{array}{l}0.69- \\
1.78 \\
\end{array}$ & 0.66 & & & & & & & & & \\
\hline & Seizures & 2.07 & $\begin{array}{l}0.92- \\
4.64\end{array}$ & 0.08 & & & & & & & & & \\
\hline
\end{tabular}




\subsection{DISCUSSION}

The aim of this study was to examine the relationship between sleep patterns and characteristics of children with ASD ranging from early to late childhood. More than $60 \%$ of children in the SSC sample had a parent identify at least one item on the SSCSI as problematic, and about $40 \%$ were classified as having a mild or moderate/severe sleep problem on the SSCSI composite or subscales. The nighttime problems subscale had the highest occurrence of sleep problems, with difficulty falling asleep being the most frequently reported problem. A considerable proportion of the sample did not get the sleep duration recommended by the National Sleep Foundation for their age (20-34\%), although only a very small proportion were reported by parents as having problems on the SSCSI sleep duration problems subscale (3\%). The number of children with mild or moderate/severe scores on the nighttime problems subscale did not decrease significantly between early and middle childhood groups, but did decrease between middle and late childhood groups. In the full sample, and in sex and age subgroups, GID and age were the variables most frequently most significantly associated with sleep problems in the SSC sample. A significantly higher mean SSCSI composite score was seen in children with NVIQ $\leq 70$. In addition, NVIQ was significantly associated with composite and nighttime problems subscales; and small interactions between age and NVIQ were present for composite problems, and nighttime problems and daytime problems subscales. Seizures were significantly correlated with increased composite and nighttime problems scores. Lower total ADOS and social affect severity scores were associated with increased odds of moderate/severe (versus mild) sleep problems on the SSCSI composite, but higher total ADOS and social affect severity scores were associated with increased odds of mild (versus none/minimal) sleep problems. However, these associations were small, had incongruous directions, and there was no significant difference in the distribution of SSCSI scores between children with low (4-5) and high (6-10) ADOS severity scores in this sample. In addition, although an interaction between age 
and total ADOS severity score was indicated, ADOS severity score was not significantly associated with sleep problems in any of the stratified groups. These inconsistencies suggest that the associations are likely false positives.

That GID was found to be frequently associated with sleep problems is consistent with results found in other studies. Comparable to Kang et al. (2014), the present study found that almost 50\% of children studied had at least one chronic GID, that rates of GID were not significantly different among age groups, and that GID was associated with sleep disturbance in children with ASD. A link between sleep problems and GID has been previously reported in the medical and ASD specific literature, with attempts to further understand this phenomenon. The first theory is that abdominal pain or discomfort is likely to make it difficult to fall asleep, stay asleep, or maintain deep sleep. Fass et al. (2000) found in their study of 505 patients with functional bowel disease and/or irritable bowel syndrome that up to $71 \%$ report sleep disturbance such as waking during the night or waking unrefreshed in the morning. In addition, individuals with ASD may have heightened pain sensitivity, compounding the effect of abdominal discomfort on sleep (Riquelme, Hatem, \& Montoya, 2016). More specifically for this population, GID in children with ASD may be due to neurobehavioral factors such as food selectivity or stool-holding behavior (Ibrahim, Voigt, Katusic, Weaver, \& William, 2009). In addition to the effect of these behaviorally-influenced GID symptoms on sleep, children who are particularly prone to restrictive, ritualistic behaviors may also exhibit bedtime problems like bedtime resistance or insistence on having a parent in the room when falling asleep.

Age was observed to be associated with sleep patterns in the way expected: negatively associated with SSCSI composite and nighttime problems subscale scores. This indicates that as children aged, the number of sleep problems reported by parents decreased. Conversely, age was significantly positively associated with moderate/severe daytime problems. Studies of sleep patterns through adolescence have shown that postpubertal children naturally tend to fall asleep and wake later than prepubertal children, and are more likely to experience difficulty waking and daytime sleepiness (M. Carskadon et al., 1979; Crowley et al., 2014; Jenni et al., 2005). Discrepancies between when adolescents naturally fall asleep, 
and when they must wake in the morning for school or work may contribute to parents reporting their older child not getting enough sleep duration. Of note, age was only associated with composite sleep problems and daytime problems in the middle childhood group, indicating that sleep patterns are changing between 9- and 13-years-old in this sample.

Lower total ADOS and social affect severity scores were found to be associated with lesser odds of mild composite sleep problems (vs. none/minimal), but greater odds of moderate/severe composite sleep problems (vs. mild). However, these associations may represent false positive results based on the size and direction of associations. First, odds ratios were small (mild: total ADOS OR $=1.07$, social affect $\mathrm{OR}=1.09$; moderate/severe: total $\mathrm{ADOS}$ OR $=0.92$, social affect $\mathrm{OR}=0.93$ ), translating to only a 7-9\% change in problems for each unit increase in ADOS severity score. That the direction of the mild vs. none/minimal and moderate vs. mild results are incongruent is concerning, as well. Second, the implication that children with less severe ASD symptoms have more reported sleep problems also does not support our hypothesis that children with more severe ASD would have more sleep problems. Indeed, there are mixed positive and negative associations between non-significant total ADOS severity score and total sleep problems (mild-severe; vs. none/minimal), mild and moderate/severe sleep problems (vs. none/minimal), and SSCSI subscale scores. Third, that total ADOS severity score was not significantly associated with sleep duration problems (irregular bedtime/waketime and no regular sleep duration) agrees with the Malow et al. (2006) finding that total ADOS is not associated parent-reported sleep duration, but does not agree with the Veatch et al. (2017) finding that shorter sleep duration was associated with more severe social impairment. Other studies, such as Zachor and Ben-Itzchak (2016) using the Autism Diagnostic Inventory-Revised (Lord et al., 1994) and the Vineland Adaptive Behavior Scales (Sparrow, Balla, Cicchetti, Harrison, \& Doll, 1984), and Hirata, Mohri, Kato-Nishimura, \& Tachibana (2016) using the Child Behavior Checklist (Achenbach, 2001) have found that children with parent-reported sleep problems had higher autism severity scores. Overall, the uncertainty surrounding the size, direction, and validity of the significant association between sleep problems and ASD severity in the 
present study and other studies indicate that more investigation into this complex relationship is warranted.

Unlike Elrod and Hood (2015), who observed that children with ASD and mild ID (FSIQ 55-70) had more trouble falling asleep than those with ASD and moderate to severe ID (FSIQ < 55), the present study did not confirm these results. The present study showed that children with NVIQ 55-70 did not have significantly more difficulty falling asleep than those with NVIQ $<55$, and they had significantly less nighttime and sleep duration problems than those with NVIQ $<55$. In fact, most of the results reported here indicated more reported sleep problems in children with lower NVIQ. Analyses showed that SSCSI composite, and nighttime problems and sleep duration problems subscale scores, were negatively associated with NVIQ. This indicates that as NVIQ decreases, the frequency of reported sleep problems increases. The present study did confirm the results of Gail-Williams et al. (2004) which showed that children with concurrent ID were found to have a greater frequency of night wakings than those with ASD and no ID.

Interactions between age and NVIQ were observed in analyses of SSCSI composite, nighttime problems, and daytime problems scores. Although the association between these interaction effects and sleep problems were small $(\mathrm{OR}=1.02-1.04)$, their frequency in the regression models may warrant further investigation. The positive direction of the interactions implies that, in the SSC sample, as age increased the effect of NVIQ on the odds of sleep problems increases. However, it is possible that the use of different IQ measures based on mental age may be creating an artificial interaction between age and NVIQ. In addition, it is becoming more evident that the genetic and environmental causes ASD and ID are varied, it is possible that there are subgroups within the SSC sample that are not accounted for in this study.

A confounding factor in the measurement and analysis of IQ in the SSC sample is the effect of ASD severity on the IQ testing process. If a child was unable to participate in part of the IQ testing because of communication or behavior difficulties, SSC researchers recorded the IQ with what they were able to measure. Consequently, some IQ scores were extremely low (NVIQ reported range: 9-152), and 
not reflective of children who would have fit the inclusion criteria for the SSC sample. Because IQ scores may be artificially low and correlated with ADOS scores, they may be creating false correlations with SSCSI scores, and in turn masking a true correlation between sleep scores and ADOS severity scores or preventing ADOS severity scores from entering the regression model. Because of this, we cannot say conclusively that ADOS severity score is or is not associated with sleep scores.

\subsubsection{Limitations}

This study has several limitations. First, little is known about administration of the SSCSI, and other sleep instruments were not used so that test-retest reliability, interrater reliability, and convergent validity cannot be calculated. Also, without an objective measure of sleep, the SSCSI was based on parents' report, and thus is subject to reporting bias. For instance, some parents may not be aware that their child is waking multiple times in the night if the child does not wake them; or children with GID or other medical comorbidities may wake their parents more often with complaints of discomfort. In addition, some parents may view symptoms such as difficulty waking in the morning or daytime sleepiness as normal for their child's developmental level, and thus not report these symptoms as problems on the SSCSI. Second, although this is one of the largest groups of children with ASD available, subgroups of the sample varied considerably in size. Because of small sample size in comparison to the full sample, females and the late childhood group did not yield reliable regression results. Also, a greater ratio of boys to girls was seen in this group (6.5:1) than that seen in the broader ASD population (4.5:1). Third, without also testing a typically developing control group, it is difficult to surmise what sleep problems are unique to ASD versus are typical in children of a certain age. 


\subsubsection{Implications}

This study advances the understanding of sleep and ASD by showing that age, and presence of GID and low NVIQ increase the risk of sleep problems in a large, well-phenotyped sample of children with ASD. Several implications may be drawn from this investigation. First, as expected, sleep problems were most prevalent in early and middle childhood, suggesting that treatments may need to focus on the sleep problems evident in younger children. However, adolescents had more daytime problems while younger children presented with more nighttime problems, indicating that treatments will need to evolve as children age. Second, using the SSC sample, this study confirmed the many prior reports of sleep problems in children with ASD. These numerous reports reinforce the need for health care professionals to screen for and discuss sleep problems with their ASD patients and families. As recommended by Malow et al. (2014), parental education in proper sleep habits should be provided. Third, since sleep problems in ASD have been associated with gastrointestinal disorders, seizures (Aldinger et al., 2015), circadian genes (Melke et al., 2008; Yang et al., 2016) and melatonin levels (Melke et al., 2008; Tordjman et al., 2005), cognitive performance (Elia et al., 2000; Limoges et al., 2013), behavior problems (Schwichtenberg et al., 2013; Sikora et al., 2012), and developmental regression (Flavia Giannotti et al., 2011), the SSC provides a wealth of opportunities to incorporate sleep into research in this large, wellcharacterized sample of children with ASD.

Several future lines of inquiry may be proposed from these results to further the understanding of sleep problems in ASD. Based on the associations found between sleep problems and GID in the present study, a next step would be to explore types of GID—constipation, diarrhea, reflux, abdominal pain, etc. It is possible that different types of GID may have distinct influences on sleep problems. In the same way, associations between various types of seizures and sleep problems may also be investigated. Additionally, the potential influence of medications on sleep problems, and on comorbidities such as GID and seizures, should be considered in future studies. The current study was not able to take into consideration the effects of medications in the current study because pertinent details, such as dose, administration time and 
frequency were not available from the parent study. To fully address the influence of medications on expression of sleep problems, variables to be collected and considered in future studies should include for each medication, the type of medication (primary mechanism of action), dose and frequency of doses, time of administration in relation to sleep time, interactions with other medications taken, and potential side effects that may themselves disturb sleep, seizure patterns, or cause GID. As age was found to be significantly associated with sleep problems, and particularly sleep problems in middle childhood, further investigation into the effect of puberty on sleep patterns in ASD is warranted. An objective measure of puberty, such as the Tanner stages, would greatly enhance our understanding of the interaction between puberty and sleep patterns.

\subsubsection{Conclusions}

In conclusion, approximately $41 \%$ of children in the SSC were categorized as having mild or moderate/severe sleep problems based on the SSCSI. Most sleep problems were seen in early and middle childhood, and the most frequent problems were in the nighttime problems subscale- bedtime problems, difficulty falling asleep, need for a parent to lay with the child, and frequent/prolonged awakenings during the night. Almost one-third of children had a reported sleep duration less than NSF recommendations. GID and age were the most frequent variables associated with of sleep problems, followed by NVIQ, sex, and age. Presence of seizures was associated with composite problems and nighttime problems. ADOS severity score is likely not associated with sleep problems in this sample. Future research into the biological basis of sleep problems and comorbidities affecting sleep, changes in sleep during puberty, and the influence of pharmacologic therapies on sleep problems may move this research into the realm of clinical interventions to improve quality of life for families affected by ASD and sleep problems. 


\title{
5.0 DATA-BASED MANUSCRIPT: VARIATIONS IN GENES RELATED TO SLEEP PATTERNS IN CHILDREN WITH AUTISM SPECTRUM DISORDERS
}

\begin{abstract}
5.1 ABSTRACT
Background: Sleep disturbance is a frequent comorbidity in children with autism spectrum disorder (ASD), estimated to affect $40-80 \%$ of cases. Previous reports have shown relationships between several circadian rhythm-related genes and sleep problems in ASD. The purpose of this study is to relate variation in and around melatonin-synthesis and suprachiasmatic nucleus genes to sleep problems in a large sample of children with ASD.

Methods: This secondary analysis used existing genotypic and phenotypic data on 2,065 children aged 418-years from the Simons Simplex Collection. Sleep problems were measured with the Simons Simplex Collection Sleep Interview. Expression quantitative trait loci and single nucleotide polymorphisms in 25 circadian genes were chosen primarily for their impact on expression levels of target genes in the brain. Associations between composite sleep problems, nighttime problems, daytime problems, and sleep duration problems, and variants were calculated using logistic regression analysis. Age, sex, nonverbal IQ, ASD severity, gastrointestinal distress, seizures, and ancestry were included as covariates. Transmission disequilibrium tests were performed to test for over-transmission of alleles in the same variants.
\end{abstract}

Results: No significant associations or transmission disequilibrium were found between gene variants and sleep problems in this sample of children with ASD. 
Conclusion: Variation in expression of investigated genes in the melatonin synthesis and suprachiasmatic nucleus pathway do not have notable impacts on sleep problems in this large sample of children with ASD. Future research may explore translational and post-translational effects of these genes, or the effects of genes in other sleep-homeostasis pathways on sleep patterns.

\subsection{INTRODUCTION}

\subsubsection{Autism spectrum disorder}

Autism spectrum disorder (ASD) is a pervasive developmental disorder characterized by deficits in social communication, and restricted, repetitive interests and behaviors (American Psychiatric Association, 2013). ASD presents early in life, persisting through the lifespan, and is termed a spectrum because symptoms of the disorder vary widely in severity. In addition to core communication and behavior difficulties, children with ASD may suffer from a range of other medical and behavioral comorbidities, such as anxiety, gastrointestinal disorders, seizures, and sleep problems. Sleep problems are estimated to affect $40-80 \%$ of children with ASD, and include difficulty falling asleep (Giannotti, Cortesi, Cerquiglini, Vagnoni, \& Valente, 2011; Goldman et al., 2017; Lambert et al., 2016) and staying asleep (Allik, Larsson, \& Smedje, 2008; Souders et al., 2009), shorter total sleep time (sleep beginning to endawakenings after sleep onset; Elrod \& Hood, 2015; Goodlin-Jones, Tang, Liu, \& Anders, 2008; Miano et al., 2007), poorer sleep efficiency (total sleep time/time in bed; Elrod \& Hood, 2015; Fletcher et al., 2016; Giannotti et al., 2011). 


\subsubsection{Genetics of sleep patterns}

Recent studies have suggested that disrupted levels of melatonin, a neurotransmitter vital in regulation of circadian rhythm, may play a role in sleep problems in ASD (Nir, Meir, Zilber, Knobler, \& Hadjez, 1995; Pagan et al., 2014; Pagan et al., 2017; Tordjman, Anderson, Pichard, Charbuy, \& Touitou, 2005). Spurred by these reports of abnormal melatonin levels in individuals with ASD, Melke et al.(2008) examined variations in ASMT in 250 individuals with ASD, and 255 controls. Two promoter polymorphisms were found significantly more frequently in individuals with ASD (rs4446909 and rs5989681, p<0.000) leading to a substantial decrease in ASMT activity $\left(p=2 \times 10^{-12}\right)$ and melatonin level $\left(p=3 \times 10^{-11}\right)$. In addition, a splice-site mutation (IVS5+2T > C) was correlated in ASD with decreased sleep efficiency (70-82\% vs. $>85 \%$ in controls) and increased night wakings (17-22 times/hr vs. $<10$ times/hr in controls). More recently, Veatch et al. (2015) confirmed a high frequency of two ASMT polymorphisms previously reported by Melke et al. (rs4446909 and rs5989681) in a group of fifteen unrelated children with ASD and sleep onset delay.

Although a number of studies have investigated links between SCN genes and risk for ASD (Chaste et al., 2011; Goto, Mizuno, Matsumoto, Yang, \& Jimbo, 2017; Hu, Sarachana, Sherrard, \& Kocher, 2015; Nicholas et al., 2007), only one study to date has examined multiple SCN genes in relation to sleep patterns in individuals with ASD. Yang et al. (2015) examined 18 genes in individuals with ASD with and without abnormal sleep patterns, and in controls. Six missense changes found exclusively in individuals with ASD and abnormal sleep problems were observed in CLOCK (c.2551A>G), MTNR1B (c.974G>A), PER3 (с.1447C>T, с.1361G>A), NIR1D1 (с.58A>C), and TIMELESS (с.1493T>C). However, mutations were also identified in ARNTL, MTNR1A, MTNR1B, PER1, PER2, PER3, and TIMELESS in individuals with ASD without sleep problems, and in PER2 in healthy controls. 


\subsubsection{Study aims}

To our knowledge, no study has examined the genes of the melatonin synthesis pathway and core SCN pathway together with characteristics of a large sample of children with ASD. Using data from the Simons Simplex Collection (SSC), a large, national ASD consortium genome-wide association study (GWAS) that also collected information about sleep patterns, the proposed study seeks to fill this gap in knowledge by relate variation in and around 25 melatonin synthesis and SCN genes to their influence on sleep problems in children with ASD. Genetic variants have been chosen for association analysis based on their impact on gene expression, as measured by RNA-Seq and single nucleotide polymorphism (SNP) data.

\subsection{METHODS}

\subsubsection{Participants}

The Simons Foundation Autism Research Initiative began the Simons Simplex Collection [SSC] with the primary goal of identifying genetic variants that contribute to ASD risk. The sample of approximately 2,750 simplex families (only one child with ASD) was recruited from 12 academic medical centers in the United States and Canada. Procedures of the original SSC study, and characteristics of the sample have been described previously. Strict phenotyping was performed on children 4- to 18-years-old with ASD using parent interview, medical history, and multiple well-validated instruments. Clinical best estimate diagnoses of autism or ASD were given based on medical history and chart review, the Autism Diagnostic Observation Schedule (ADOS; Lord et al., 1989) and Autism Diagnostic Interview-Revised (ADI-R; Lord, Rutter, \& Le Couteur, 1994) scores, and the Diagnostic and Statistical Manual of Mental 
Disorders-IV-Text Revised (DSM-IV-TR; American Psychiatric Association, 2013; Simons Foundation Autism Research Initiative, 2014).

\subsubsection{Procedures}

Procedures of the parent study, inclusion and exclusion criteria, and characteristics of the SSC study sample have been described previously (Fischbach \& Lord, 2010; Frazier et al., 2014; Johansson, Feeley, et al., n.d.; Lord et al., 2012). The current study was a secondary analysis of existing genotypic and phenotypic data from the Simons Foundation. Informed consent was obtained for the SSC study from parents or caregivers, and assent was obtained from children as required by local institutional review boards. Approval was obtained from the University of Pittsburgh Institutional Review Board for the current study. Data was de-identified by the Simons Foundation prior to receipt by the present researchers.

Phenotypic data was obtained from the Simons Foundation software platform, SFARI Base. Demographic data (age, sex, etc.) were collected when families entered the SSC study. As previous analysis of the sample (Johansson et al., n.d.), revealed associations between sleep problems and presence or absence of gastrointestinal distress (GID), non-verbal IQ (NVIQ), and Autism Diagnostic Observation Scale (ADOS) total score, these variables, with the addition of seizures, sex, age, and ancestry, will be used as covariates in analyses. GID was defined as parent reports of bloating/excess gas, celiac disease, constipation, diarrhea, ulcers, gastroesophageal reflux disease, inflammatory bowel disease (Crohn's disease, ulcerative colitis), irritable bowel syndrome, abdominal pain, unusual stools, vomiting, and/or other GID; and seizures as parent reports atonic drop, infantile spasms; or partial complex, grand mal, petit mal, simple partial, and/or other seizures. Presence or absence of GID and seizures was determined using data from the medical history interview conducted by the parent study at baseline. Four normreferenced instruments were used to measure NVIQ in the SSC sample based on chronologic and mental age: the Differential Ability Scales-II (DAS-II; Elliott, 2012; PsychCorp, 2016), Weschler Intelligence 
Scale for Children-Fourth Edition (WISC-IV; Weschler, 2003), Wechsler Abbreviated Scale of Intelligence (WASI; Wechsler, 2011), and Mullen Scales of Early Learning (MSEL; Bradley-Johnson, 1997). The ADOS (Gotham, Pickles, \& Lord, 2009; Lord et al., 1989) is a semi-structured observational instrument used with other instruments for diagnosis of ASD and classification of severity, and measures social responsiveness, communication, language delay, and spatial ability.

\subsubsection{Instruments}

Simons Simplex Collection Sleep Interview (SSCSI): The SSCSI (Johansson, Rohay, \& Chasens, in press) is an 11-item cross-sectional, parent-report questionnaire evaluating nighttime and daytime sleep problems, and sleep duration problems (Table 10). Items are scored as 0 for "no" and 1 for "yes”. A continuous composite score is made by summing the 10 dichotomous items, plus 1 point for reported sleep duration outside National Sleep Foundation recommendations based on the child's age (Hirshkowitz et al., 2015). Composite scores range from 0 to 11, with higher scores indicating worse sleep. Subscale scores are determined by summing the items within the subscale (Nighttime problems and Daytime problems range $=0-4$; Sleep duration problems range $=0-2$. Participants were excluded from the present study if more than two items were missing or coded as unknown.

\subsubsection{Gene variant selection}

Expression quantitative trait loci (eQTLs), or genomic variants that influence the expression of one or more genes, were chosen primarily using gene expression data from the CommonMind Consortium (CMC; Fromer et al., 2016). The CMC used RNA-Seq data from dorsolateral prefrontal (DLPFC) brain tissue from adults with and without neuropsychiatric disorders (Albert \& Kruglyak, 2015). Variants were also taken from the Genotype-Tissue Expression project (GTEx; The GTEx Consortium, 2015), 
HaploReg v.4 (Ward \& Kellis, 2016), and variants identified by other groups as affecting sleep patterns in individuals with ASD (Melke et al., 2008; Veatch et al., 2015; Yang et al., 2016).

For the current study, gene expression was analyzed in cis- (within $1 \mathrm{Mb}$ of gene) variants with a false discovery rate threshold FDR $\leq 0.05$, revealing that 16 of the 25 genes were expressed in substantial quantities in the CMC DLPFC data (TPH2, CLOCK, ARNTL, PER1/2/3, BHLHE40/41, DBP, GAD1/2, $N R 1 D 2, R O R A / B, C S N K 1 D / E$ ). Data was used from the GTEx project (The GTEx Consortium, 2015) to identify eQTLs for 6 of the 7 remaining genes (MTNR1B, CRY1, DDC, AANAT, PROK2, CIART, CRY1/2, TIMELESS). For MTNR1A, 2,149 variants were found in the Haploreg v.4 program using the location of the gene plus 50KB on each side (chr4:187404809-187526537). Of these, 12 variants had data for expression. Those eQTLs with a $p$-value $<0.001$ were chosen, and the independent pairwise function in Plink was used to prune the list based on linkage disequilibrium (LD) and an $r^{2}=0.5$. Proxy eQTLs were required for some eQTLs that were not available in the SSC. SNAP Proxy Search was used to find proxy eQTLs (Broad Institute, 2015). These processes resulted in 73 eQTLs from the CMC; 49 were available in SSC data (32 proxy eQTL in significant LD were substituted for eQTLs not in SSC data). Of the 40 eQTLs from GTEx, 16 were available in the SSC data (12 proxy eQTLs were substituted). Haploreg identified 8 eQTLs; 3 were available in SSC data (1 proxy eQTL was used). Of 26 variants identified by previous literature in 10 genes (ASMT, CLOCK, CSNK1D/E, MTNR1A/B, NR1D1, PER1/2/3, TIMELESS), 4 were available in the SSC data (2 proxies were used): ASMT rs4446909 was identified by Melke et al. (2008) and Veatch et al. (2015); PER2 rs934945, PER3 rs10462020 (proxy: rs10462021), and PER3 rs228697 (proxy: rs228690) were identified by Yang et al. (2016). In all, 73 independent eQTLs or variants were identified $(\mathrm{CMC}=50, \mathrm{GTEx}=16$, Haploreg= 3, literature $=4$; Table 12).

Individuals in the SSC sample were genotyped using either Illumina 1Mv1 (approximately 325 families), Illumina 1Mv3 Duo (approximately 1,160 families), or Illumina HumanOMNI 2.5 (approximately 625 families) arrays. The same array version was used to analyze all members of a family. 


\subsubsection{Statistical analyses}

SSCSI items, composite and subscale scores, and sex were described using frequency counts and percentages. Parent reported regular sleep duration (hours per night) and age (years) were described using mean, standard deviation, and range. Regular sleep duration less than 3 standard deviations below the population mean, were replaced with the 3rd standard deviation below the mean (4.85 hours). MannWhitney U was used to compare SSCSI composite and subscale scores between age groups, and chisquare analyses to compare between sexes. Descriptive and correlational analyses were performed with SPSS version 23 (SPSS Inc., Chicago, IL).

Power analysis was conducted using the Genetic Power Calculator (Purcell, Cherny, \& Sham, 2003). Power to detect factors associated with sleep patterns was calculated assuming 72 independent tests, resulting in a Bonferroni corrected significance level of $0.05 / 72$ or $p=0.0007$. Given the large size of the SSC database, the present study was well-powered $(\geq 80 \%)$ to detect an OR of 1.2 for a common variant (risk allele frequency $=0.5$ ) or a less common variant (risk allele frequency $=0.25$; $p$-value $=0.0007$ ).

\subsubsection{Logistic regression analysis}

SSCSI composite and subscale scores were dichotomized into none/minimal (scores 0-1) or sleep problems present $(\geq 2)$. Logistic regression analyses were performed to estimate associations between SSCSI composite and factor scores, and individual variant reference allele dosage (0, 1, 2 alleles). HardyWeinberg equilibrium (HWE) was assessed in participants of non-Hispanic European Ancestry using the HardyWeinberg package in the $\mathrm{R}$ suite (R Core Team, 2016) and a $p<0.0007$ (Bonferroni correction). All variants were in HWE. All models were adjusted for age (years), ADOS severity score (range: 4-10), sex (male=0, female=1), NVIQ $(<40=1,40-54=2,55-70=3,71-100=4,>100=5)$, GID (no=0, yes=1), seizures (no=0, yes=1), and ancestry (1=non-Hispanic European, 2=Asian, 3=Hispanic European, 4=African American), and were performed under the additive genetic model ((0, 1, 2 alleles). Analyses were performed by building models with no sleep problems versus sleep problems (mild or 
moderate/severe) as the outcome variable. Odds ratios with $95 \%$ confidence intervals were used to measure the strength of associations between each gene variant and sleep patterns. Logistic regression was performed with the MASS package in the $\mathrm{R}$ Suite. Level of significance was set at $p<0.0007$ (Bonferroni correction).

\subsubsection{Transmission disequilibrium test}

The transmission disequilibrium test (TDT), one of several family-based association methods, examines expected and observed transmission of parental genotypes or alleles to offspring affected by a disease or trait. Transmission disequilibrium occurs when there is non-random or greater than expected transmission of a certain allele to offspring with a trait or disease (Laird \& Lange, 2011). The TDT was performed with parent-child trios in which the child had SSCSI composite and subscale sleep problems present using Bioconductor trio package in the R Suite (Schwender et al., 2015). Level of significance was set at $p<0.0007$ (Bonferroni correction). Only variants in which more than 100 trios were tested are reported.

\subsection{RESULTS}

\subsubsection{Sample characteristics}

Characteristics of the sample are presented in Table 9. Of the full sample of children with genetic data available ( $\mathrm{N}=2,200), 135$ were excluded for missing sleep data. Three-quarters of these (78-80\%) were also missing descriptive data (age, ancestry, diagnosis, ADOS severity score, NVIQ, sleep duration) so that comparisons between children included and excluded from the sample were not feasible. There was no significant difference in sex between those included and excluded $(p=0.42)$. The sample was

predominantly male (87\%), Non-Hispanic European (78\%), did not have seizures (95\%), and had NVIQ above 70 (75\%). Exactly half of the sample had GID. The mean age of the sample was approximately 9- 
years (range: 4-18-years). Children below the mean age were significantly more likely than children over the mean age to have a NVIQ $<70$ (40.2\% vs. $33.0 \%, p<0.05)$, to have seizures (6.9\% vs. $4.3 \%, p<0.01)$, and to have sleep problems on the SSCSI composite $(76.3 \%$ vs. $61.6 \%, p<0.01)$ and nighttime sleep problem subscale $(38.2 \%$ vs. $30.7 \%, p<0.05)$. Younger children also had significantly longer sleep duration than older children (mean $=9.5(1.3)$ hrs vs. 8.8(1.2)hrs, $p<0.01$ ). In addition, no significant differences were found between males and females in regular sleep duration, age, proportion of children with GID, seizures, NVIQ $\leq$ 70, or sleep duration less than recommended for age. Significantly more females had NVIQ $\leq 70$ (37.6\% vs. 23.7\%, $p<0.01)$. 
Table 9: Sample Characteristics

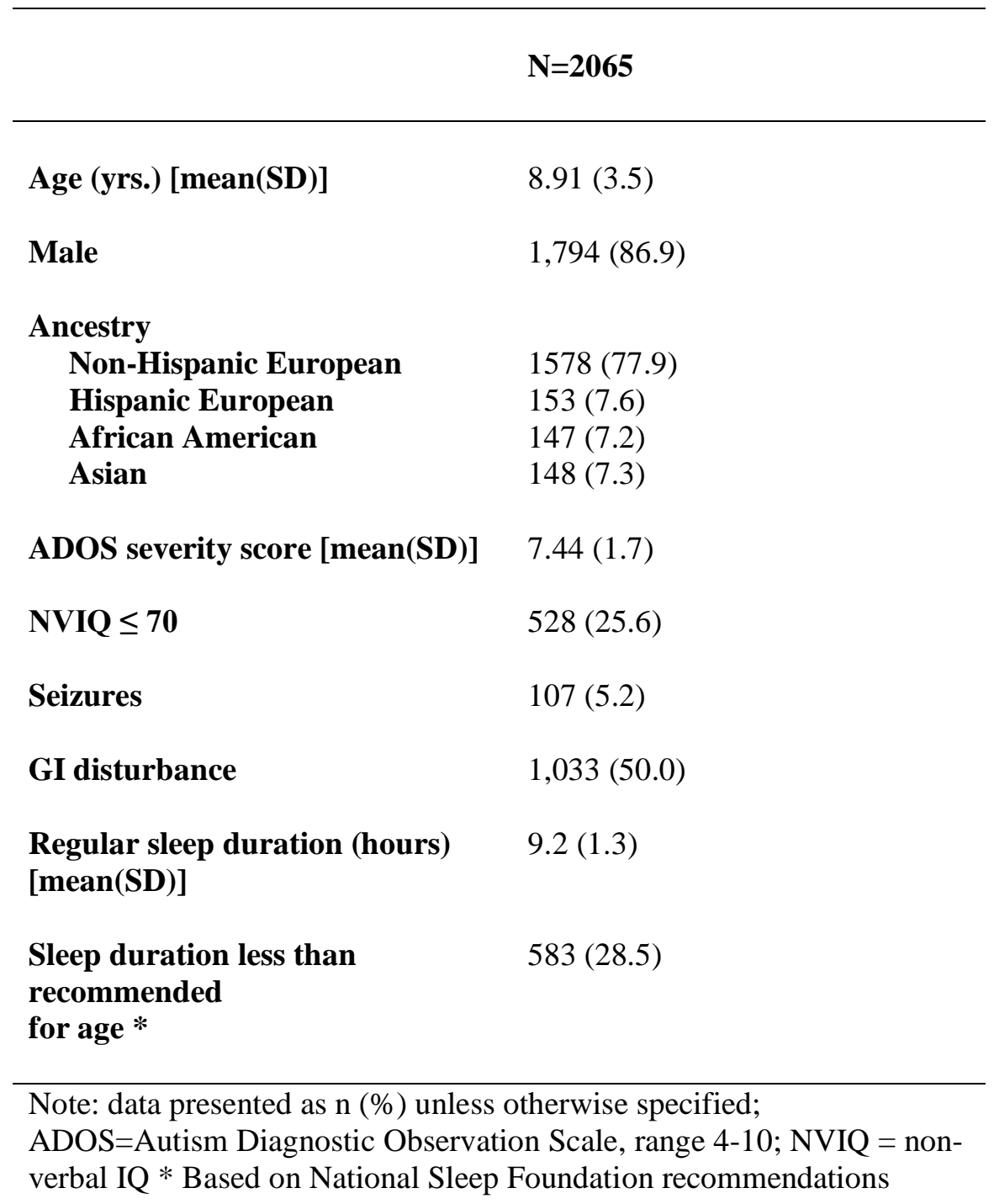

\subsubsection{Genotype associations with SSCSI sleep problems}

While no genetic associations remained significant after applying correction for multiple testing, due to the hypothesis-generating nature of the study, results that meet the conventional $p<0.05$ cut-off will be discussed. Those eQTLs associated $(p<0.05)$ with sleep problems (mild or moderate/severe vs. none) based on SSCSI composite and subscale scores are listed in Table 15. Composite sleep problems were 
most highly associated with the CRY1 eQTL, rs1035768 (reference allele: A, OR $=1.58,95 \%$ CI: 1.07 2.34); and also with $R O R A$ rs8038644 (reference allele: A, OR $=1.23,95 \%$ CI: $1.07-1.42$ ) and the GAD1 eQTL rs16857804 (reference allele: A, OR = 1.15, 95\% CI: 1.00-1.31). Nighttime sleep problems were most highly associated with the MTNR1B eQTL, rs1962041 (reference allele: G, OR= 1.44, 95\% CI: 1.09-1.89); and also associated with PER2 eQTLs rs6431588 (reference allele: A, OR=1.26, 95\% CI: 1.05-1.52) and rs934945 (reference allele: $\mathrm{G}, \mathrm{OR}=1.25,95 \% \mathrm{CI}: 1.04-1.50$ ), and the RORA eQTL rs8038664 (reference allele: A, OR = 1.23, 95\% CI: 1.04-1.44). Daytime problems were most highly associated with the MTNR1B eQTL, rs11598 (reference allele: G, OR = 1.59, 95\% CI: 1.17-2.17); and also associated with the GAD1 rs16857804 (reference allele: A, OR = 1.16, 95\% CI: 1.00-1.33) and the CLOCK eQTL rs11943456 (reference allele: G, OR = 1.14, 95\% CI: 1.00-1.30). Sleep duration problems were most highly associated with the GAD2 eQTL, rs11014824 (reference allele: G, OR = 4.47, 95\% CI: 1.08-18.56); and also associated with the $D D C$ rs3735274 (reference allele: G, OR = 3.14, 95\% CI: 1.06 9.31) and the GAD2 eQTL rs7077123 (reference allele: G, OR = 1.96, 95\% CI: 1.04-3.69). 
Table 10: Genotype Associations with SSCSI Composite and Subscale Sleep Problems

\begin{tabular}{|c|c|c|c|c|c|c|c|c|}
\hline & Variant & Gene & $\begin{array}{c}\text { Reference } \\
\text { allele }\end{array}$ & b & OR & $\begin{array}{c}95 \% \\
\text { CI } \\
\text { lower }\end{array}$ & $\begin{array}{c}95 \% \\
\text { CI } \\
\text { upper }\end{array}$ & $p$-value* \\
\hline \multirow{3}{*}{$\begin{array}{l}\text { Composite } \\
\text { sleep problems }\end{array}$} & rs1035768 & CRY1 & A & 0.458 & 1.58 & 1.07 & 2.34 & 0.022 \\
\hline & rs8038664 & RORA & A & 0.208 & 1.23 & 1.07 & 1.42 & 0.004 \\
\hline & rs16857804 & $G A D 1$ & A & 0.137 & 1.15 & 1.00 & 1.31 & 0.049 \\
\hline \multirow{4}{*}{$\begin{array}{l}\text { Nighttime } \\
\text { problems }\end{array}$} & rs1962041 & MTNR1B & $\mathrm{G}$ & 0.363 & 1.44 & 1.09 & 1.89 & 0.009 \\
\hline & rs6431588 & PER2 & A & 0.235 & 1.26 & 1.05 & 1.52 & 0.011 \\
\hline & rs934945 & PER2 & G & 0.222 & 1.25 & 1.04 & 1.50 & 0.017 \\
\hline & rs8038664 & RORA & A & 0.204 & 1.23 & 1.04 & 1.44 & 0.013 \\
\hline \multirow{3}{*}{$\begin{array}{l}\text { Daytime } \\
\text { problems }\end{array}$} & rs11598 & MTNR1B & G & 0.466 & 1.59 & 1.17 & 2.17 & 0.003 \\
\hline & rs16857804 & $G A D 1$ & A & 0.146 & 1.16 & 1.00 & 1.33 & 0.044 \\
\hline & rs11943456 & CLOCK & G & 0.133 & 1.14 & 1.00 & 1.30 & 0.048 \\
\hline \multirow{3}{*}{$\begin{array}{l}\text { Sleep duration } \\
\text { problems }\end{array}$} & rs11014824 & $G A D 2$ & $\mathrm{G}$ & 1.498 & 4.47 & 1.08 & 18.56 & 0.039 \\
\hline & rs3735274 & $D D C$ & G & 1.143 & 3.14 & 1.06 & 9.31 & 0.039 \\
\hline & rs7077123 & GAD2 & G & 0.673 & 1.96 & 1.04 & 3.69 & 0.037 \\
\hline
\end{tabular}

* No statistically significant results after Bonferroni correction $(p<0.0007)$; Top results among the variants tested are displayed. OR = odds ratio

\subsubsection{Transmission disequilibrium test}

No variants showed statistically significant over-transmission of alleles from parents to children with sleep problems after adjustment for multiple testing ( $p<0.0007$; Table 16). Sleep duration problems did not have any variants with $>100$ trios. The variant associated with the highest odds of sleep problems in the TDT analysis was CIART rs28730724, which displayed over-transmission of the A allele in children with composite sleep problems (OR=1.71, 95\% CI: 1.16-2.52, $p=0.012)$. RORA rs4775351 displayed over-transmission of the A allele in children with composite and daytime problems (OR=1.20, 95\% CI: 1.01-1.42, $p=0.038$; OR=1.23, 95\% CI: 1.01-1.48, $p=0.037$ ). Over-transmission of the A allele was shown in two variants of CSNK1E: rs135721 with composite sleep problems (OR=1.17, 95\% CI: 1.01-1.37, 
$p=0.041)$ and rs4296527 with daytime problems (OR=1.24, 95\% CI: $1.03-1.49, p=0.023)$. Two CLOCK variants were associated with transmission of alleles to children with daytime problems: rs693367 showed over-transmission of the A allele (OR=1.21, 95\% CI: 1.03-1.43, $p=0.019)$, and rs11943456 of the $\mathrm{G}$ allele (OR=1.23, 95\% CI: 1.05-1.43, $p=0.009)$. GAD1 rs13395562 and MTNR1B rs11598 also showed overtransmission of the $\mathrm{G}$ allele in children with daytime problems (OR=1.21, 95\% CI: 1.03-1.42, $p=0.018$; OR=1.55, 95\% CI: 1.08-2.24, $p=0.019$, respectively). Only one variant, PER2 rs6431588, showed overtransmission to children with nighttime problems (A allele, OR=1.36, 95\% CI: 1.09-1.71, $p=0.007$ ).

\subsection{DISCUSSION}

This study represents the first analysis of relationships between variation in genes of both the melatonin synthesis and core SCN pathways and sleep problems in children with ASD from the SSC. To investigate these associations, logistic regression was performed on 72 variants (68 eQTLs and 4 SNPs) in 25 genes and sleep problems-SSCSI composite problems, nighttime problems, daytime problems, and sleep duration problems-controlling for age, sex, total ADOS severity score, NVIQ, presence of gastrointestinal distress and seizures, and ancestry. To corroborate association findings, transmission disequilibrium was investigated between parents and children affected by sleep problems. The analysis revealed no variants significantly associated with sleep problems, and no evidence was found for overtransmission of alleles from parents to children with ASD affected by sleep problems. It can be said with some confidence that eQTLs and SNPs for the investigated genes in the melatonin synthesis pathway and core SCN pathways do not have a notable impact on sleep problems in children with ASD.

A number of strengths lend credence to the results of this study. While many previous studies have been limited in their ability to identify meaningful associations between genomic and biochemical variants and sleep patterns by small sample sizes and choice of only one or two gene, the present study presents a comprehensive analysis of 25 genes with more than 2,000 strictly phenotyped children. A 
unique aspect of this study is the a priori selection of eQTLs that affect transcription of these genes in the brain. A majority of eQTLs chosen came from the CMC, which mined gene expression data from the brain tissue, including tissue from individuals with schizophrenia, a neurobehavioral disorder with genetic similarities to ASD (O’Connell, Mcgregor, Lochner, Emsley, \& Warnich, 2018; The Autism Spectrum Disorders Working Group of The Psychiatric Genomics Consortium, 2017). The innovative use of RNASeq data to select gene variants affecting target gene expression may help to explain the divergent results from those of other groups who chose variants based on previous literature (Veatch, Pendergast, et al., 2015) or whole exome sequencing (Chaste et al., 2010; Melke et al., 2008; Yang et al., 2016). While these groups looked at associations with structural mutations in the genes or post-translational melatonin levels, the present study investigated associations with variation in transcription, or gene expression. The size of the SSC sample was also a key strength of this study, even with a large number of tests performed. This study was powered to detect effects of approximately $1 \%$ of variability in sleep problems yet did not detect significant effects of gene variants after correction for multiple testing. The use of TDT also augments power by eliminating the problem of population stratification.

The present study had several limitations. As neither the CMC nor GTEx collected samples with consideration to timing of circadian gene expression, the choice of eQTLs may not capture markers that were not expressed at the time of sample collection. The CMC used samples from the brains of adults, which may exhibit different gene expression patterns than the brains of children. In addition, using secondary data precludes the use of different sleep questionnaires or measuring levels of hormones related to sleep patterns, like melatonin and cortisol. Conversely, studies such as this one require large samples making it almost impossible to muster such a sample for which hormones were measured directly. The present study had a large sample, but if the effects of the variants were small, the current sample size might not be adequate to detect them. Finally, the SSCSI was developed for use with the SSC, and has not been independently validated or used with other samples of children with ASD. The SSCSI was also based on parents' report, and thus may be subject to reporting bias. 


\subsubsection{Implications}

Continued research into the genomics of sleep problems in individuals with ASD is necessary. The lack of association found between expression of genes and sleep patterns in the present study does not imply that the melatonin synthesis and SCN pathway should be abandoned. Although this study showed that transcription of these genes was not influencing sleep patterns, the effect of translational and posttranslational modification on melatonin and SCN proteins cannot be dismissed. That a number of research studies have shown low melatonin or melatonin metabolite levels (Leu et al., 2011; Melke et al., 2008; Tordjman et al., 2005), and success with melatonin supplementation (Cuomo et al., 2017; Gringras et al., 2017; Wright et al., 2011) demonstrates this is still a valid research area. However, as other gene pathways in the brain and body, such as glutamate, GABA, and serotonin are known to interact with melatonin and the SCN, inquiries should not be limited to the melatonin/SCN pathway.

\subsubsection{Conclusion}

Variation in the expression of core genes in the melatonin synthesis and SCN pathway were not found to have notable impacts on sleep problems in this large sample of children with ASD based on logistic regression and transmission disequilibrium tests. The results of this study may differ from previous investigations of the genetics of sleep problems in ASD due to the innovative use of expression data to guide gene variant selection, and because of the much larger size of the current sample. This study may be of use in clarifying genomic mechanisms related to the melatonin/SCN pathway and sleep problems in ASD, and in proposing further lines of inquiry. Future research may explore translational and posttranslational effects of these genes, the effects of genes in other sleep-homeostasis pathways on sleep patterns, and the use of objective measures of sleep. 
APPENDIX A

COMPARISON OF SLEEP PATTERNS IN CHILDREN WITH ASD AND TYPICALLY DEVELOPING CHILDREN 
Table 11: Comparison of Sleep Patterns in Children with ASD and Typically Developing Children

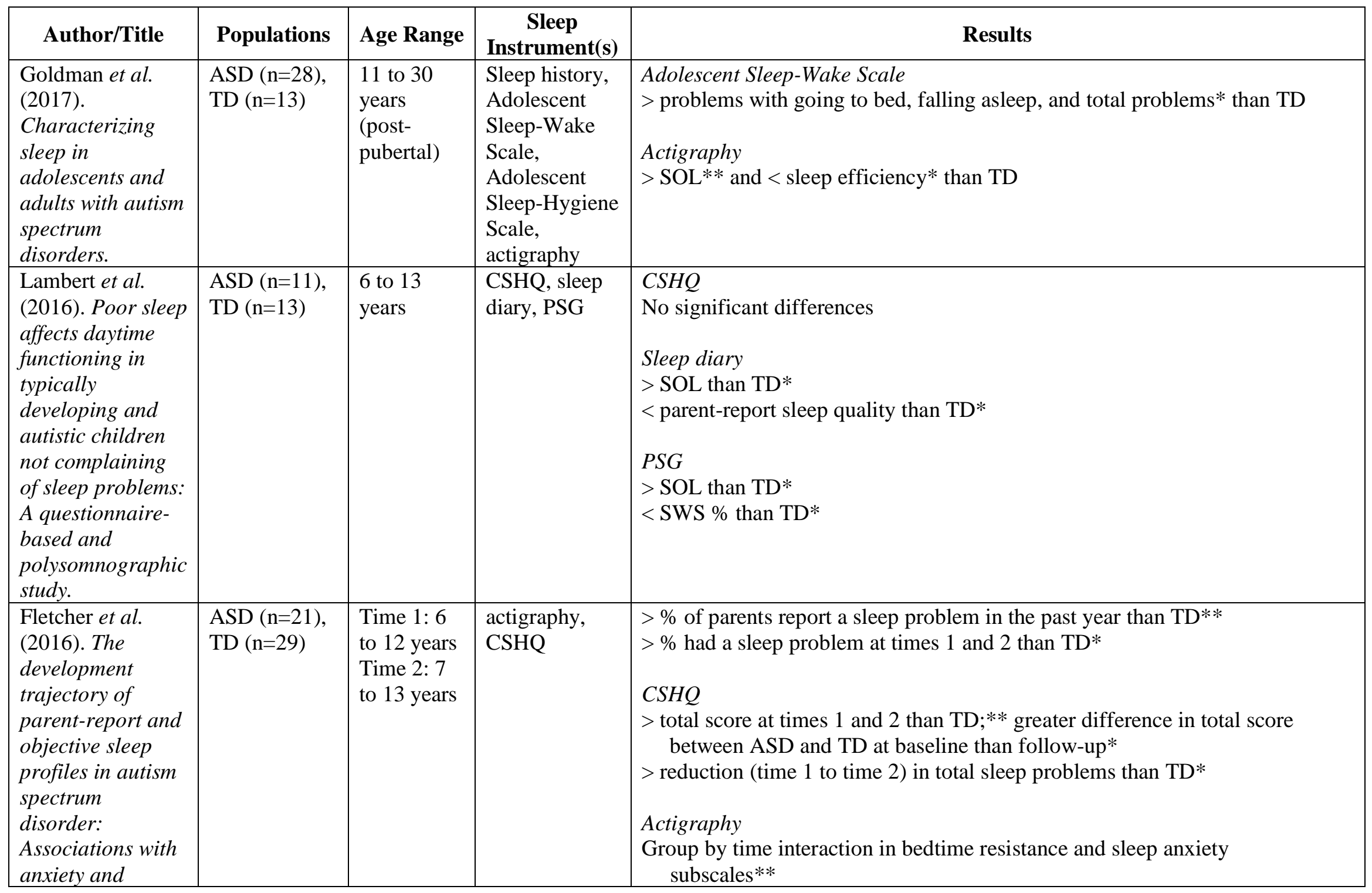




\begin{tabular}{|c|c|c|c|c|}
\hline bedtime routines. & & & & $\begin{array}{l}<\text { average (times } 1 \text { and } 2 \text { ) SE \% than TD** } \\
>\text { variability (time } 1 \text { to time } 2 \text { ) WASO (decreased)** and SE (decreased)* } \\
\text { than TD }\end{array}$ \\
\hline $\begin{array}{l}\text { May et al. (2015). } \\
\text { Sleep in high- } \\
\text { functioning } \\
\text { children with } \\
\text { autism: } \\
\text { Longitudinal } \\
\text { developmental } \\
\text { change and } \\
\text { associations with } \\
\text { behavior } \\
\text { problems. }\end{array}$ & $\begin{array}{l}\text { ASD }(n=46) \\
\operatorname{TD}(n=38)\end{array}$ & $\begin{array}{l}7 \text { to } 12 \\
\text { years }\end{array}$ & CSHQ & $\begin{array}{l}>\text { total score }{ }^{\text {a }} \text { at baseline and } 1 \text { year later** than TD } \\
>\text { bedtime resistance, }{ }^{*} \text { sleep onset delay, }{ }^{* *} \text { sleep duration, }{ }^{* *} \text { sleep } \\
\text { anxiety,* parasomnia,* daytime sleepiness** subscales than TD }\end{array}$ \\
\hline $\begin{array}{l}\text { Elrod and Hood } \\
\text { (2015; meta- } \\
\text { analysis). Sleep } \\
\text { differences among } \\
\text { children with } \\
\text { autism spectrum } \\
\text { disorders and } \\
\text { typically } \\
\text { developing peers: } \\
\text { A meta-analysis. }\end{array}$ & $\begin{array}{l}\text { ASD } \\
(\mathrm{n}=343), \mathrm{TD} \\
(\mathrm{n}=221) \\
(10 \text { papers })\end{array}$ & $\begin{array}{l}2 \text { to } 19 \\
\text { years }\end{array}$ & $\begin{array}{l}\text { actigraphy, } \\
\text { PSG }\end{array}$ & $\begin{array}{l}\text { Relationship between ASD and: TST moderated by ID,** SE by } \\
\text { medications, } * \text { SE by method (actigraphy, PSG)* } \\
\text { Pooled difference in means, ASD vs. TD: TST }=-32.8 \text { minutes, } 95 \% \text { CI: } \\
\quad-49.0 \text { to }-16.6) \text {, SL }=10.9 \text { minutes ( } 95 \% \text { CI: } 6.7 \text { to } 15.0), \text { SE }=-1.9 \% \\
\text { (95\% CI: }-3.1 \text { to }-0.7)\end{array}$ \\
\hline $\begin{array}{l}\text { Hodge } \text { et al. } \\
\text { (2014). Sleep } \\
\text { patterns in } \\
\text { children with and } \\
\text { without autism } \\
\text { spectrum } \\
\text { disorders: } \\
\text { Developmental } \\
\text { comparisons. } \\
\end{array}$ & $\begin{array}{l}\text { ASD } \\
(n=108), T D \\
(n=108)\end{array}$ & $\begin{array}{l}3 \text { to } 17 \\
\text { years }\end{array}$ & $\begin{array}{l}\text { CSHQ, sleep } \\
\text { diary }\end{array}$ & $\begin{array}{l}\text { CSHQ } \\
>\text { total score;** and bedtime resistance, }{ }^{* *} \text { sleep onset delay,** sleep } \\
\text { duration, }{ }^{* *} \text { sleep anxiety, }{ }^{* *} \text { night waking, }{ }^{* *} \text { sleep disordered } \\
\text { breathing* subscales than TD } \\
>\text { total score than TD in children 6-9 years* and } 10-17 \text { years* } \\
\text { Sleep diary } \\
<\text { sleep duration than TD* } \\
>\text { minutes awake after sleep onset than TD* }\end{array}$ \\
\hline $\begin{array}{l}\text { Giannotti et al. } \\
\text { (2011). Sleep in } \\
\text { children with }\end{array}$ & $\begin{array}{l}\text { ASD } \\
\text { (regressive } \\
\mathrm{n}=18\end{array}$ & $\begin{array}{l}5 \text { to } 10 \\
\text { years }\end{array}$ & $\begin{array}{l}\text { Sleep diary, } \\
\text { CSHQ, PSG }\end{array}$ & $\begin{array}{l}\text { Sleep diary } \\
\text { Regressive: later bedtime and earlier rise time, < sleep time, > SOL than } \\
\text { TD;** later bedtime, }<\text { sleep time, }{ }^{* *}>\text { SOL than non-regressed* }\end{array}$ \\
\hline
\end{tabular}




\begin{tabular}{|c|c|c|c|c|}
\hline $\begin{array}{l}\text { autism with and } \\
\text { without autistic } \\
\text { regression. }\end{array}$ & $\begin{array}{l}\text { nonregressive } \\
\mathrm{n}=22) \mathrm{TD} \\
(\mathrm{n}=12)\end{array}$ & & & $\begin{array}{l}\text { Non-regressive: later bedtime, < sleep time, > SOL than TD** } \\
\text { CSHQ } \\
\text { Regressive: > total score; and bedtime resistance, sleep onset delay, sleep } \\
\text { duration, night waking subscales than TD** and non-regressed** } \\
\text { Non-regressive: > total score; and bedtime resistance, sleep onset delay, } \\
\text { sleep duration, night waking subscales than TD** } \\
\text { PSG } \\
\text { Regressive: < TST, SE than TD and non-regressive;* < SWS \%, REM \% } \\
\text { than TD** > SOL, WASO \%, REM \% than TD and non-regressive;* } \\
\text { >awakenings/hr, REM latency, stage } 2 \% \text { than TD* } \\
\text { Non-regressive: }<\text { TST, SE than TD;* > SOL, WASO \%, awakenings/hr, } \\
\text { REM latency than TD }\end{array}$ \\
\hline $\begin{array}{l}\text { Cotton and } \\
\text { Richdale (2010). } \\
\text { Sleep patterns and } \\
\text { behaviour in } \\
\text { typically } \\
\text { developing } \\
\text { children and } \\
\text { children with } \\
\text { autism, Down } \\
\text { syndrome, } \\
\text { Prader-Willi } \\
\text { syndrome and } \\
\text { intellectual } \\
\text { disability. }\end{array}$ & $\begin{array}{l}\text { ASD }(n=34), \\
\text { TD }(n=33)\end{array}$ & $\begin{array}{l}3 \text { to } 16 \\
\text { years }\end{array}$ & Sleep diary & $\begin{array}{l}\text { Daytime problems } \\
\text { Behavior problems: > than } \mathrm{TD}^{* *} \\
\text { Nighttime problems } \\
\text { Level of bedtime sleepiness: < than TD** } \\
\text { Bedtime behavioral problems: > than } \mathrm{TD}^{* *}\end{array}$ \\
\hline $\begin{array}{l}\text { Buckley et al. } \\
\text { (2010). REM } \\
\text { sleep percentage } \\
\text { in children in } \\
\text { autism compared } \\
\text { with } \\
\text { developmental } \\
\text { delay and typical }\end{array}$ & $\begin{array}{l}\text { ASD }(n=60) \\
\operatorname{TD}(n=15)\end{array}$ & $\begin{array}{l}2 \text { to } 13 \\
\text { years }\end{array}$ & PSG & $<$ TST, SWS percentage, REM percentage than TD** \\
\hline
\end{tabular}




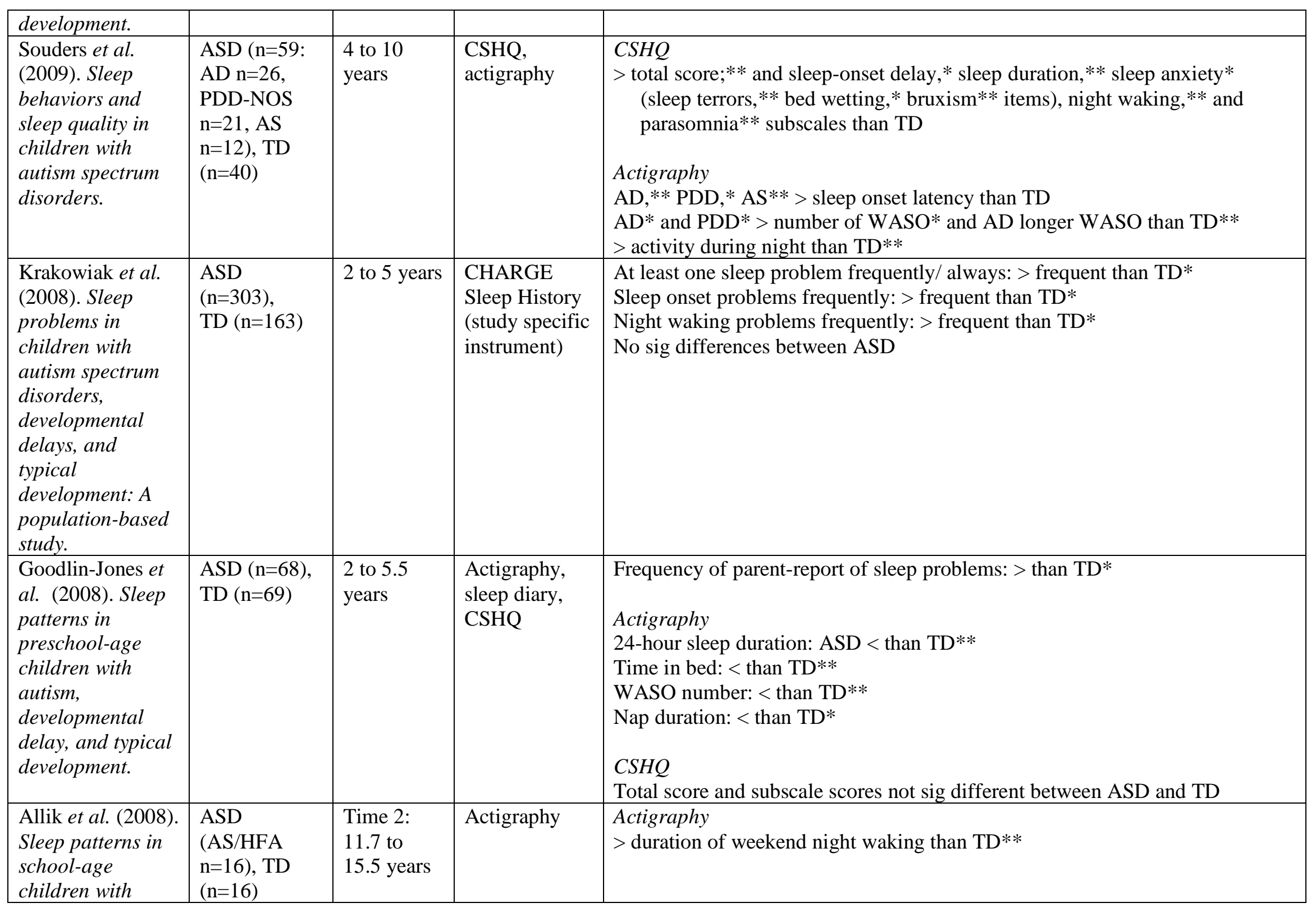




\begin{tabular}{|c|c|c|c|c|}
\hline $\begin{array}{l}\text { Asperger } \\
\text { syndrome or high- } \\
\text { functioning } \\
\text { autism: A follow- } \\
\text { up study. }\end{array}$ & & & & \\
\hline $\begin{array}{l}\text { Giannotti et al. } \\
\text { (2008). An } \\
\text { investigation of } \\
\text { sleep } \\
\text { characteristics, } \\
\text { EEG } \\
\text { abnormalities and } \\
\text { epilepsy in } \\
\text { developmentally } \\
\text { regressed and } \\
\text { non-regressed } \\
\text { children with } \\
\text { autism. }\end{array}$ & $\begin{array}{l}\text { ASD } \\
\text { (regressive } \\
n=70, \text { non- } \\
\text { regressive } \\
n=34), \text { TD } \\
(n=162)\end{array}$ & $\begin{array}{l}2.5 \text { to } 8 \\
\text { years }\end{array}$ & $\begin{array}{l}\text { CSHQ, sleep } \\
\text { diary, sleep } \\
\text { history }\end{array}$ & $\begin{array}{l}\text { Sleep diary } \\
\text { Regressive: later bedtime, earlier rise time, < TST, > SOL than TD** and } \\
\text { non-regressive;** > SOL than non-regressed* } \\
\text { Non-regressive: later bedtime, <TST, > SOL than TD** } \\
\text { CSHQ } \\
\text { Regressive: > bedtime resistance, sleep onset delay, sleep duration, night } \\
\text { wakings subscale scores than TD** and non-regressive;** > sleep } \\
\text { anxiety, parasomnia,** daytime sleepiness* subscale scores than TD } \\
\text { Non-regressive: > bedtime resistance, sleep onset delay, sleep duration, } \\
\text { sleep anxiety, night-wakings, parasomnia, }{ }^{* *} \text { daytime sleepiness* } \\
\text { subscale scores than TD } \\
\text { Sleep history } \\
\text { Regressive: > percent sleep problems before age 2, mean age at onset of } \\
\text { sleep problems, \% behavioral insomnia of childhood diagnosis, \% } \\
\text { circadian rhythm sleep disorder-delayed sleep phase type and irregular } \\
\text { sleep/wake type, \% seasonal sleep variations than TD;** > mean age at } \\
\text { onset of sleep problems, } * * \% \text { circadian rhythm sleep disorder-delayed } \\
\text { sleep phase type and irregular sleep/wake type, unclassified sleepiness } \\
\text { than non-regressive* } \\
\text { Non-regressive: > \% sleep problems before age 2, mean age at onset of } \\
\text { sleep problems, \% behavioral insomnia of childhood, \% circadian } \\
\text { rhythm sleep disorder-delayed sleep phase type and irregular } \\
\text { sleep/wake type, \% seasonal sleep variations than TD** }\end{array}$ \\
\hline $\begin{array}{l}\text { Bruni et al. } \\
\text { (2007). Sleep } \\
\text { architecture and } \\
\text { NREM alterations } \\
\text { in children and } \\
\text { adolescents with }\end{array}$ & $\begin{array}{l}\text { ASD }(n=10), \\
\operatorname{AS}(n=8), T D \\
(n=12)\end{array}$ & $\begin{array}{l}7 \text { to } 15 \\
\text { years }\end{array}$ & $\begin{array}{l}\text { PSG, sleep } \\
\text { questionnaire }\end{array}$ & AS: > sleep stage shifts than ASD* and TD* \\
\hline
\end{tabular}




\begin{tabular}{|c|c|c|c|c|}
\hline $\begin{array}{l}\text { Asperger } \\
\text { syndrome. }\end{array}$ & & & & \\
\hline
\end{tabular}


APPENDIX B

MANUSCRIPT \#1 SUPPLEMENTARY TABLES 


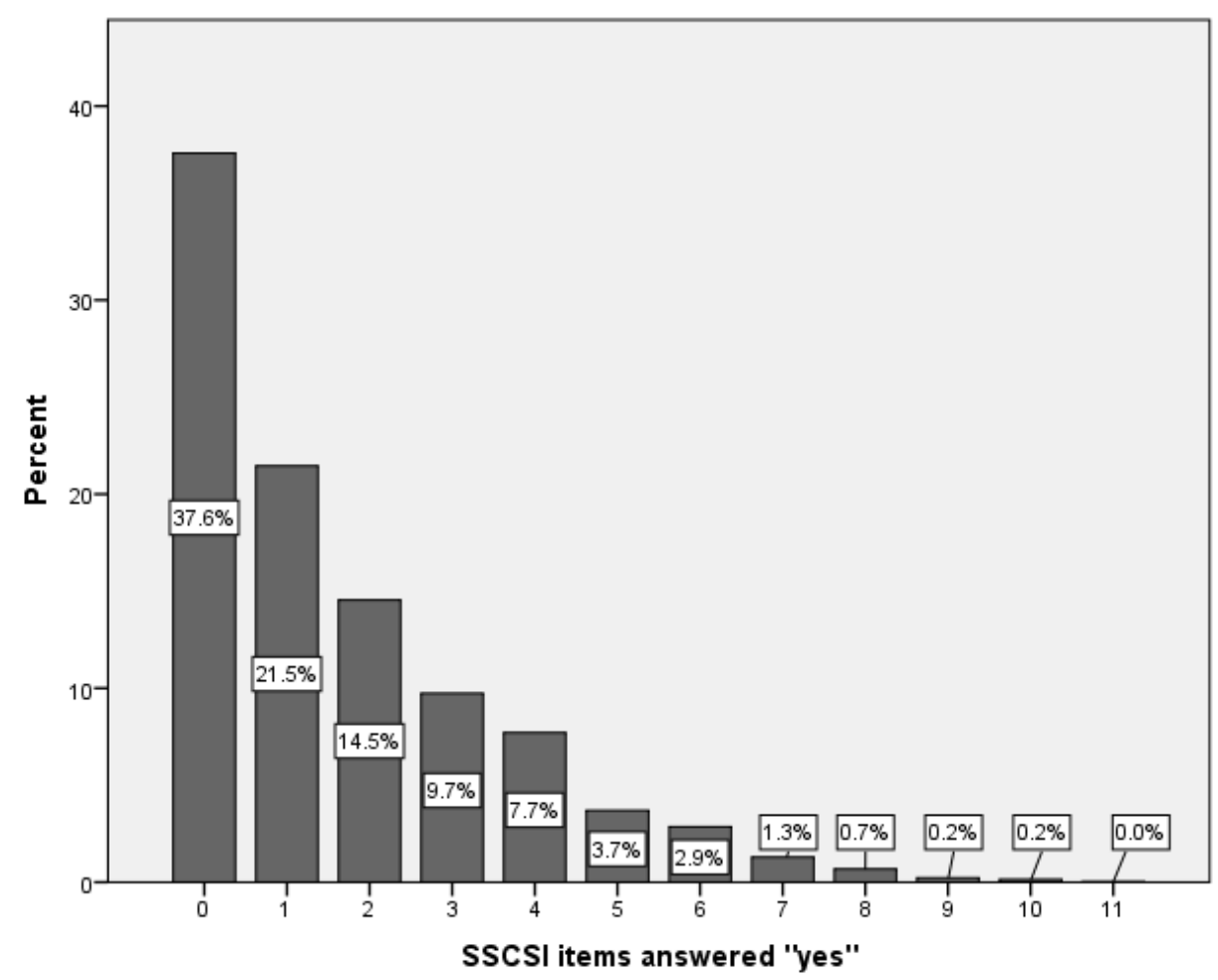

Figure 7: Distribution of SSCSI Item Scores

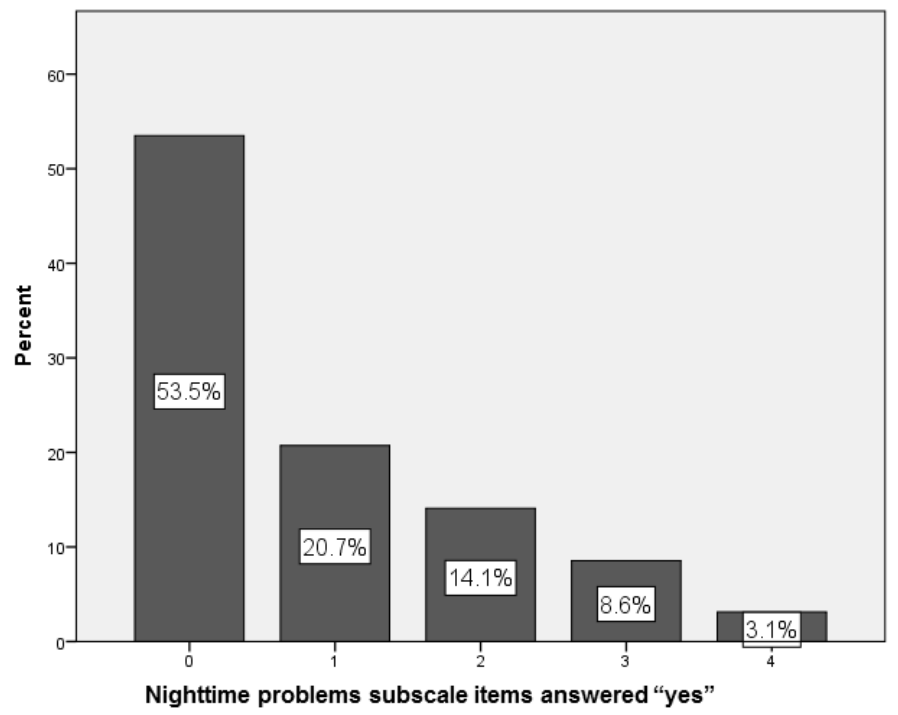

Figure 8: Distribution of SSCSI Nighttime Problems Item Scores 


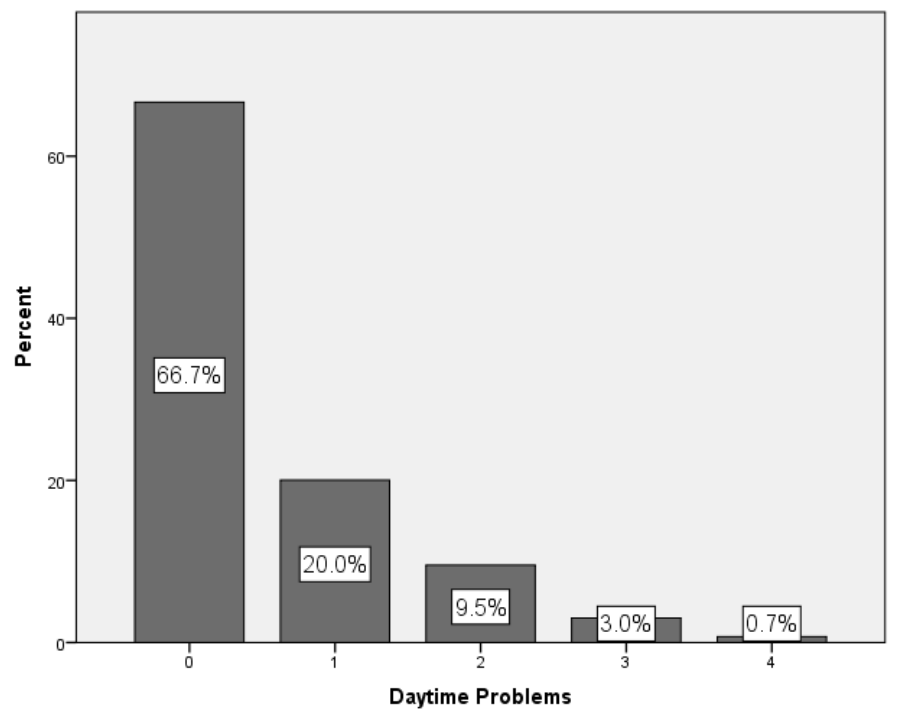

Figure 9: Distribution of SSCSI Daytime Problems Item Scores

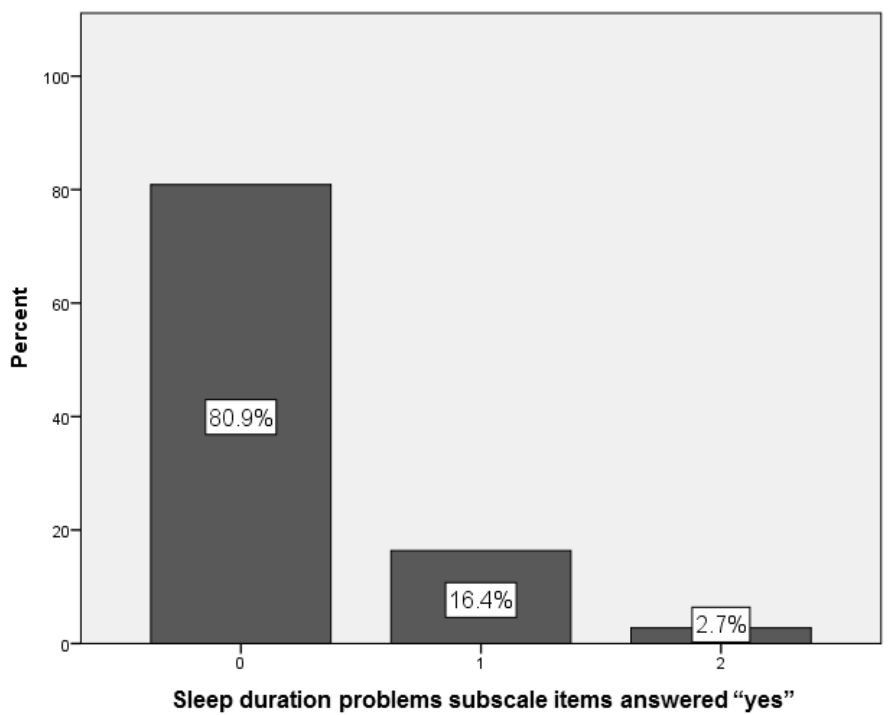

Figure 10: Distribution of Sleep Duration Problems Item Scores 
APPENDIX C

SIMONS SIMPLEX COLLECTION SLEEP INTERVIEW (SSCSI) 
Table 12. Simons Simplex Collection Sleep Interview (SSCSI)

$\underline{\text { Subscales }}$

Nighttime

problems

Day time

problems

Sleep duration

problems
Does your child currently have:

1. difficulty going to bed?

2. difficulty falling asleep?

3. need for a parent to lay down with them to fall asleep?

4. frequent or prolonged awakenings at night?

5. difficulty waking up in the morning?

6. long or frequent naps?

7. sleepiness during the day?

8. enough sleep?

9. an irregular bedtime and waketime?

10. a regular sleep duration?

11. What is your child's regular sleep duration in hours? 
APPENDIX D

IQ AND AUTISM SEVERITY MEASURE PSYCHOMETRICS 
Table 13: IQ and Autism Severity Measure Psychometrics

\begin{tabular}{|c|c|c|}
\hline $\begin{array}{l}\text { Weschler Intelligence Scale for } \\
\text { Children-IV }\end{array}$ & $\begin{array}{l}\text { Reliability: full scale } \\
\text { "Special” groups } \\
\text { Test-retest: full scale }\end{array}$ & $\begin{array}{l}r=0.97 \\
r=0.82-0.93 \\
r=0.93\end{array}$ \\
\hline $\begin{array}{l}\text { Weschler Abbreviated Scale of } \\
\text { Intelligence }\end{array}$ & $\begin{array}{l}\text { Reliability: full scale } \\
\text { Subscales } \\
\text { Psychiatric sample } \\
\text { Test-retest: full scale } \\
\text { Subscales }\end{array}$ & $\begin{aligned} r & =0.87-0.91 \\
r & =0.92-0.96 \\
r & =0.96-0.98 \\
r & =0.87-0.95 \\
r & =0.79-0.90\end{aligned}$ \\
\hline Mullen Scale of Early Learning & $\begin{array}{l}\text { Reliability: full scale } \\
\text { Test-retest: subscales }\end{array}$ & $\begin{array}{l}r=0.83-0.95 \\
r=0.85-0.96\end{array}$ \\
\hline Differential Ability Scale-II & $\begin{array}{l}\text { Reliability: full scale } \\
\text { Subscales }\end{array}$ & $\begin{array}{l}r=0.94-0.96 \\
r=0.87-0.96\end{array}$ \\
\hline $\begin{array}{l}\text { Autism Diagnostic Observation } \\
\text { Scale }\end{array}$ & $\begin{array}{l}\text { Internal consistency: } \\
\text { Social affect } \\
\text { Repetitive behavior } \\
\text { Sensitivity: } \\
\text { Specificity: } \\
\text { Test-retest: }\end{array}$ & $\begin{array}{l}\alpha=0.87-0.92 \\
\alpha=0.51-0.66 \\
91-98 \% \\
84-94 \% \\
r=0.59-0.82\end{array}$ \\
\hline
\end{tabular}


APPENDIX E

VARIANTS INCLUDED IN GENETIC REGRESSION ANALYSIS 
Table 14: Variants Included in Genetic Regression Analysis

\begin{tabular}{|c|c|c|c|c|c|}
\hline Variant & $\begin{array}{l}\text { Proxy for } \\
\text { Variant* }\end{array}$ & $\begin{array}{c}\text { Allele } \\
\text { Frequency } \\
\text { [n (\%)] }\end{array}$ & HWE & Gene & Source \\
\hline $\begin{array}{l}\text { rs12939873 } \\
\text { n }=2055\end{array}$ & & $\begin{array}{ll}\text { AA } & 807(39.1) \\
\text { AG } & 939(45.5) \\
\text { GG } & 309(15.0) \\
\end{array}$ & $p=0.5793$ & $A A N A T$ & GTEx \\
\hline rs3760138 & $\begin{array}{c}\mathrm{rs} 1000821 \\
\mathrm{n}=2065\end{array}$ & $\begin{array}{cc}\text { AA } & 695(33.7) \\
\text { AG } & 1016(49.2) \\
\text { GG } & 354(17.1) \\
\end{array}$ & $p=0.2627$ & $A A N A T$ & GTEX \\
\hline rs59467367 & $\begin{array}{l}\mathrm{rs} 4788926 \\
\mathrm{n}=613\end{array}$ & $\begin{array}{cc}\text { AA } & 255(41.6) \\
\text { AG } & 286(46.7) \\
\text { GG } & 72(11.7) \\
\end{array}$ & $p=0.3773$ & AANAT & GTEX \\
\hline rs10832178 & $\begin{array}{c}\text { rs11023081 } \\
\mathrm{n}=2064\end{array}$ & $\begin{array}{ll}\text { AA } & 968(46.9) \\
\text { AG } & 903(43.7) \\
\text { GG } & 193(9.3)\end{array}$ & $p=0.1745$ & ARNTL & CMC \\
\hline $\begin{array}{r}\text { rs4446909 } \\
\mathrm{n}=1152\end{array}$ & & $\begin{array}{cc}\text { AA } & 666(57.8) \\
\text { AG } & 411(35.7) \\
\text { GG } & 75(6.5)\end{array}$ & $p=0.2982$ & $A S M T$ & $\begin{array}{c}\text { Melke, } \\
2008\end{array}$ \\
\hline rs11710676 & $\begin{array}{l}\text { rs12629173 } \\
n=2063\end{array}$ & $\begin{array}{lr}\text { GG } & 528(25.6) \\
\text { GA } & 1004(48.6) \\
\text { AA } & 531(25.7)\end{array}$ & $p=0.4501$ & BHLHE40 & CMC \\
\hline rs36126004 & $\begin{array}{c}\text { rs6786132 } \\
n=1451\end{array}$ & $\begin{array}{cc}\text { AA } & 965(66.5) \\
\text { AG } & 433(29.8) \\
\text { GG } & 53(3.7) \\
\end{array}$ & $p=0.773$ & BHLHE40 & CMC \\
\hline rs4685806 & $\begin{array}{c}\text { rs7651990 } \\
n=2065\end{array}$ & $\begin{array}{lr}\text { GG } & 1330(64.4) \\
\text { GA } & 625(30.3) \\
\text { AA } & 110(5.3) \\
\end{array}$ & $p=0.4157$ & BHLHE40 & CMC \\
\hline $\begin{array}{r}\text { rs931503 } \\
n=2060\end{array}$ & & $\begin{array}{lc}\text { GG } & 1544(74.8) \\
\text { GA } & 469(22.7) \\
\text { AA } & 47(2.3)\end{array}$ & $p=0.3342$ & BHLHE40 & CMC \\
\hline rs4246238 & $\begin{array}{c}\text { rs } 4963647 \\
n=2062\end{array}$ & $\begin{array}{cc}\text { GG } & 1838(89.0) \\
\text { GA } & 217(10.5) \\
\text { AA } & 7(0.3) \\
\end{array}$ & $p=0.6351$ & BHLHE41 & CMC \\
\hline $\begin{array}{l}\text { rs28730724 } \\
\text { n=1451 }\end{array}$ & & $\begin{array}{cc}\text { AA } & 1290(88.9) \\
\text { AG } & 158(10.9) \\
\text { GG } & 3(0.2) \\
\end{array}$ & $p=0.6182$ & CIART & GTEx \\
\hline rs4549456 & $\begin{array}{c}\text { rs11943456 } \\
n=2065\end{array}$ & $\begin{array}{lr}\text { GG } & 538(26.1) \\
\text { GA } & 1033(50.0) \\
\text { AA } & 494(23.9) \\
\end{array}$ & $p=0.7625$ & CLOCK & CMC \\
\hline rs482114 & $\begin{array}{r}\text { rs693367 } \\
n=2056\end{array}$ & $\begin{array}{ll}\text { GG } & 973(47.1) \\
\text { GA } & 879(42.6) \\
\text { AA } & 204(9.9)\end{array}$ & $p=0.8602$ & CLOCK & CMC \\
\hline rs12298001 & $\begin{array}{c}\text { rs4964521 } \\
n=2063\end{array}$ & $\begin{array}{ll}\text { AA } & 723(35.0) \\
\text { AG } & 950(46.0) \\
\text { GG } & 390(18.9)\end{array}$ & $p=0.157$ & CRY1 & GTEX \\
\hline rs4964471 & $\begin{array}{c}\text { rs1035768 } \\
\mathrm{n}=613\end{array}$ & $\begin{array}{lc}\text { AA } & 467(76.2) \\
\text { AG } & 136(22.2) \\
\text { GG } & 10(1.6)\end{array}$ & $p=0.9999$ & CRY1 & GTEX \\
\hline
\end{tabular}




\begin{tabular}{|c|c|c|c|c|c|}
\hline rs57707013 & $\begin{array}{c}\text { rs11150805 } \\
n=2064\end{array}$ & $\begin{array}{cc}\text { AA } & 1453(70.4) \\
\text { AG } & 560(27.1) \\
\text { GG } & 51(2.5)\end{array}$ & $p=0.7862$ & CSNK1D & CMC \\
\hline rs135729 & $\begin{array}{r}\mathrm{rs} 135721 \\
\mathrm{n}=2064\end{array}$ & $\begin{array}{lc}\text { AA } & 1078(52.2) \\
\text { AG } & 811(39.3) \\
\text { GG } & 175(8.5) \\
\end{array}$ & $p=0.2057$ & CSNK1E & CMC \\
\hline $\begin{array}{c}\text { rs11089862 } \\
n=2064\end{array}$ & & $\begin{array}{ll}\text { AA } & 619(30.0) \\
\text { AG } & 992(48.0) \\
\text { GG } & 453(21.9)\end{array}$ & $p=0.9596$ & CSNK1E & CMC \\
\hline $\begin{array}{c}\text { rs4296527 } \\
\text { n=1451 }\end{array}$ & & $\begin{array}{ll}\text { GG } & 392(27.0) \\
\text { GA } & 727(50.1) \\
\text { AA } & 332(22.9) \\
\end{array}$ & $p=0.8093$ & CSNK1E & CMC \\
\hline $\begin{array}{c}\text { rs5756931 } \\
\text { n=2060 }\end{array}$ & & $\begin{array}{ll}\text { GG } & 781(37.8) \\
\text { GA } & 963(46.6) \\
\text { AA } & 316(15.3)\end{array}$ & $p=0.8752$ & CSNK1E & CMC \\
\hline rs526744 & $\begin{array}{r}\text { rs837642 } \\
n=2064\end{array}$ & $\begin{array}{lc}\text { GG } & 1094(53.0) \\
\text { GA } & 828(40.1) \\
\text { AA } & 142(6.9) \\
\end{array}$ & $p=0.0961$ & $D B P$ & CMC \\
\hline $\begin{array}{c}\text { rs11575281 } \\
n=2063\end{array}$ & & $\begin{array}{lc}\text { CC } & 1937(93.8) \\
\text { CA } & 126(6.1) \\
\text { AA } & 0 \\
\end{array}$ & $p=0.2593$ & $D D C$ & GTEx \\
\hline rs2122817 & $\begin{array}{l}\text { rs11575548 } \\
n=2065\end{array}$ & $\begin{array}{lr}\text { AA } & 1645(79.7) \\
\text { AC } & 407(19.7) \\
\text { CC } & 13(0.6)\end{array}$ & $p=0.039$ & $D D C$ & GTEx \\
\hline rs1157375 & $\begin{array}{c}\text { rs7809758 } \\
n=2064\end{array}$ & $\begin{array}{ll}\text { GG } & 853(41.3) \\
\text { GA } & 971(47.0) \\
\text { AA } & 240(11.6) \\
\end{array}$ & $p=0.0672$ & $D D C$ & GTEx \\
\hline rs58129743 & $\begin{array}{c}\text { rs3807553 } \\
n=2062\end{array}$ & $\begin{array}{cc}\text { AA } & 1671(80.9) \\
\text { AG } & 379(18.4) \\
\text { GG } & 12(0.6) \\
\end{array}$ & $p=0.2090$ & $D D C$ & GTEx \\
\hline rs6952023 & $\begin{array}{c}\text { rs3735274 } \\
n=1451\end{array}$ & $\begin{array}{lc}\text { GG } & 804(55.4) \\
\text { GA } & 551(38.0) \\
\text { AA } & 96(6.6)\end{array}$ & $p=0.8696$ & $D D C$ & GTEx \\
\hline rs6963996 & $\begin{array}{c}\text { rs3807558 } \\
n=2065\end{array}$ & $\begin{array}{lr}\text { AA } & 1253(60.7) \\
\text { AG } & 710(34.4) \\
\text { GG } & 102(4.9) \\
\end{array}$ & $p=0.8812$ & $D D C$ & GTEx \\
\hline rs10930411 & $\begin{array}{l}\text { rs10930412 } \\
n=2065\end{array}$ & $\begin{array}{cc}\text { CC } & 1629(78.9) \\
\text { CA } & 404(19.6) \\
\text { AA } & 32(1.5) \\
\end{array}$ & $p=0.883$ & $G A D 1$ & CMC \\
\hline rs11684294 & $\begin{array}{c}\text { rs11903215 } \\
\mathrm{n}=1451\end{array}$ & $\begin{array}{ll}\text { AA } & 410(28.3) \\
\text { AC } & 711(49.0) \\
\text { CC } & 330(22.7) \\
\end{array}$ & $p=0.589$ & $G A D 1$ & CMC \\
\hline $\begin{array}{c}\text { rs13014896 } \\
\text { n=2065 }\end{array}$ & & $\begin{array}{l}\text { CC } 876(42.4) \\
\text { CA } 928(44.9) \\
\text { AA } 261(12.6)\end{array}$ & $p=0.9569$ & $G A D 1$ & CMC \\
\hline $\begin{array}{c}\text { rs13395562 } \\
n=2063\end{array}$ & & $\begin{array}{l}\text { GG } 906(43.9) \\
\text { GA } 908(44.0) \\
\text { AA } 249(12.1) \\
\end{array}$ & $p=0.9543$ & $G A D 1$ & CMC \\
\hline rs13414831 & $\begin{array}{c}\text { rs16857804 } \\
n=2063\end{array}$ & $\begin{array}{l}\text { GG } 986(47.7) \\
\text { GA } 869(42.1)\end{array}$ & $p=0.6356$ & $G A D 1$ & CMC \\
\hline
\end{tabular}




\begin{tabular}{|c|c|c|c|c|c|}
\hline & & AA $208(10.1)$ & & & \\
\hline $\begin{array}{l}\text { rs17607321 } \\
n=2062\end{array}$ & & $\begin{array}{lc}\text { CC } & 1731(83.8) \\
\text { CA } & 310(15.0) \\
\text { AA } & 21(1.0) \\
\end{array}$ & $p=0.8295$ & $G A D 1$ & CMC \\
\hline rs34375966 & $\begin{array}{c}\text { rs7568852 } \\
n=2064\end{array}$ & $\begin{array}{l}\text { CC } 761(36.9) \\
\text { CA } 985(47.7) \\
\text { AA } 318(15.4) \\
\end{array}$ & $p=0.6011$ & $G A D 1$ & CMC \\
\hline rs56851249 & $\begin{array}{l}\mathrm{rs} 13384194 \\
\mathrm{n}=2060\end{array}$ & $\begin{array}{l}\text { CC } 902(43.7) \\
\text { CA } 920(44.6) \\
\text { AA } 238(11.5) \\
\end{array}$ & $p=0.8659$ & $G A D 1$ & CMC \\
\hline rs62170724 & $\begin{array}{c}\text { rs } 4668263 \\
n=2064\end{array}$ & $\begin{array}{cc}\text { AA } & 1302(63.1) \\
\text { AG } & 663(32.1) \\
\text { GG } & 99(4.8) \\
\end{array}$ & $p=0.1277$ & $G A D 1$ & CMC \\
\hline rs6759322 & $\begin{array}{c}\text { rs1364529 } \\
n=2064\end{array}$ & $\begin{array}{l}\text { AA } 698(33.8) \\
\text { AG } 980(47.5) \\
\text { GG } 386(18.7)\end{array}$ & $p=0.232$ & $G A D 1$ & CMC \\
\hline $\begin{array}{r}\text { rs7564575 } \\
\mathrm{n}=2065\end{array}$ & & $\begin{array}{cc}\text { AA } & 1560(75.5) \\
\text { AG } & 464(22.5) \\
\text { GG } & 41(2.0) \\
\end{array}$ & $p=0.412$ & $G A D 1$ & CMC \\
\hline rs11014816 & $\begin{array}{l}\text { rs11014824 } \\
\mathrm{n}=2061\end{array}$ & $\begin{array}{cc}\text { AA } & 1713(83.0) \\
\text { AG } & 328(15.9) \\
\text { GG } & 20(1.0) \\
\end{array}$ & $p=0.149$ & $G A D 2$ & CMC \\
\hline rs11015040 & $\begin{array}{l}\text { rs7077123 } \\
\text { n=2060 }\end{array}$ & $\begin{array}{ll}\text { AA } & 887(43.0) \\
\text { AG } & 926(44.8) \\
\text { GG } & 247(12.0) \\
\end{array}$ & $p=0.822$ & $G A D 2$ & CMC \\
\hline rs2442858 & $\begin{array}{c}\text { rs2442861 } \\
\mathrm{n}=1150\end{array}$ & $\begin{array}{lc}\text { CC } & 878(76.3) \\
\text { CA } & 258(22.4) \\
\text { AA } & 14(1.2) \\
\end{array}$ & $p=0.4713$ & $G A D 2$ & CMC \\
\hline rs787041 & $\begin{array}{r}\text { rs787033 } \\
n=2064\end{array}$ & $\begin{array}{ll}\text { GG } & 740(35.8) \\
\text { GA } & 959(46.4) \\
\text { AA } & 365(17.7) \\
\end{array}$ & $p=0.9562$ & $G A D 2$ & CMC \\
\hline rs9299827 & $\begin{array}{c}\text { rs2252096 } \\
n=2062\end{array}$ & $\begin{array}{ll}\text { GG } & 982(47.6) \\
\text { GA } & 890(43.1) \\
\text { AA } & 190(9.2) \\
\end{array}$ & $p=0.3029$ & $G A D 2$ & CMC \\
\hline $\begin{array}{l}\mathrm{rs} 10013543 \\
\mathrm{n}=2063\end{array}$ & & $\begin{array}{ll}\text { AA } & 584(28.3) \\
\text { AC } & 972(47.1) \\
\text { CC } & 507(24.6) \\
\end{array}$ & $p=0.0869$ & MTNR1A & Haploreg \\
\hline $\begin{array}{c}\text { rs } 1317237 \\
\mathrm{n}=1450\end{array}$ & & $\begin{array}{ll}\text { GG } & 802(55.3) \\
\text { GA } & 544(37.5) \\
\text { AA } & 104(7.2) \\
\end{array}$ & $p=0.3939$ & MTNR1A & Haploreg \\
\hline rs767168 & $\begin{array}{c}\text { rs7675885 } \\
n=2064\end{array}$ & $\begin{array}{lr}\text { CC } & 513(24.8) \\
\text { CA } & 1046(50.7) \\
\text { AA } & 505(24.5) \\
\end{array}$ & $p=0.1904$ & MTNR1A & Haploreg \\
\hline rs7950811 & $\begin{array}{l}\text { rs11598 } \\
n=2053\end{array}$ & $\begin{array}{lc}\text { GG } & 1871(90.6) \\
\text { GA } & 178(8.6) \\
\text { AA } & 4(0.2) \\
\end{array}$ & $p=0.7919$ & MTNR1B & GTEX \\
\hline rs16919041 & $\begin{array}{c}\text { rs1962041 } \\
\mathrm{n}=2064\end{array}$ & $\begin{array}{cc}\text { AA } & 1722(83.4) \\
\text { AG } & 327(15.8) \\
\text { GG } & 15(0.7) \\
\end{array}$ & $p=0.7431$ & MTNR1B & GTEX \\
\hline
\end{tabular}




\begin{tabular}{|c|c|c|c|c|c|}
\hline rs11714673 & $\begin{array}{c}\text { rs2043581 } \\
n=2062\end{array}$ & $\begin{array}{ll}\text { AA } & 657(31.8) \\
\text { AC } & 982(47.6) \\
\text { CC } & 423(20.5) \\
\end{array}$ & $p=0.877$ & NR1D2 & CMC \\
\hline $\begin{array}{r}\text { rs7619728 } \\
\mathrm{n}=2061\end{array}$ & & $\begin{array}{lc}\text { AA } & 1824(88.3) \\
\text { AG } & 230(11.1) \\
\text { GG } & 7(0.3)\end{array}$ & $p=0.8145$ & NR1D2 & CMC \\
\hline rs55739732 & $\begin{array}{c}\text { rs1294525 } \\
n=1150\end{array}$ & $\begin{array}{lc}\text { GG } & 756(65.7) \\
\text { GA } & 354(30.8) \\
\text { AA } & 40(3.5)\end{array}$ & $p=0.8355$ & PER1 & CMC \\
\hline rs6729088 & $\begin{array}{c}\text { rs6431588 } \\
n=2065\end{array}$ & $\begin{array}{lc}\text { AA } & 1397(67.7) \\
\text { AG } & 608(29.4) \\
\text { GG } & 60(2.9)\end{array}$ & $p=0.5591$ & PER2 & CMC \\
\hline $\begin{array}{r}\text { rs934945 } \\
n=2062\end{array}$ & & $\begin{array}{lc}\text { AA } & 1302(63.1) \\
\text { AG } & 681(33.0) \\
\text { GG } & 79(3.8)\end{array}$ & $p=0.5251$ & PER2 & $\begin{array}{l}\text { Yang et al, } \\
2015\end{array}$ \\
\hline rs10462020 & $\begin{array}{l}\text { rs10462021 } \\
n=2062\end{array}$ & $\begin{array}{lc}\text { GG } & 1398(67.7) \\
\text { GA } & 613(29.7) \\
\text { AA } & 51(2.5) \\
\end{array}$ & $p=0.0789$ & PER3 & $\begin{array}{l}\text { Yang et al, } \\
2015\end{array}$ \\
\hline rs228697 & $\begin{array}{r}\text { rs228690 } \\
n=2064\end{array}$ & $\begin{array}{lc}\text { AA } & 1749(84.7) \\
\text { AG } & 303(14.7) \\
\text { GG } & 12(0.6)\end{array}$ & $p=0.9999$ & PER3 & $\begin{array}{l}\text { Yang et al, } \\
2015\end{array}$ \\
\hline rs58654499 & $\begin{array}{c}\text { rs9310221 } \\
n=2063\end{array}$ & $\begin{array}{ll}\text { AA } & 704(34.1) \\
\text { AG } & 995(48.2) \\
\text { GG } & 364(17.6)\end{array}$ & $p=0.599$ & PROK2 & GTEX \\
\hline $\begin{array}{c}\text { rs10519085 } \\
n=2064\end{array}$ & & $\begin{array}{ll}\text { GG } & 633(30.7) \\
\text { GA } & 963(46.6) \\
\text { AA } & 468(22.7) \\
\end{array}$ & $p=0.1303$ & $R O R A$ & CMC \\
\hline rs28868703 & $\begin{array}{c}\text { rs8038513 } \\
n=2041\end{array}$ & $\begin{array}{cc}\text { GG } & 1198(58.0) \\
\text { GA } & 759(36.8) \\
\text { AA } & 84(4.1)\end{array}$ & $p=0.0168$ & $R O R A$ & CMC \\
\hline $\begin{array}{c}\text { rs17204945 } \\
\text { n=2063 }\end{array}$ & & $\begin{array}{cc}\text { AA } & 1452(70.3) \\
\text { AG } & 558(27.0) \\
\text { GG } & 53(2.6)\end{array}$ & $p=0.5147$ & $R O R A$ & CMC \\
\hline rs34088848 & $\begin{array}{c}\text { rs4419005 } \\
n=2065\end{array}$ & $\begin{array}{ll}\text { AA } & 1230(59.6) \\
\text { AG } & 719(34.8) \\
\text { GG } & 116(5.6)\end{array}$ & $p=0.6684$ & $R O R A$ & CMC \\
\hline $\begin{array}{c}\text { rs } 4775351 \\
n=2064\end{array}$ & & $\begin{array}{l}\text { GG } 1347(65.2) \\
\text { GA } \\
622(30.1) \\
\text { AA }\end{array}$ & $p=0.851$ & RORA & CMC \\
\hline rs55870008 & $\begin{array}{l}\text { rs10851691 } \\
n=2065\end{array}$ & $\begin{array}{ll}\text { AA } & 522(25.3) \\
\text { AG } & 1033(50.0) \\
\text { GG } & 510(24.7)\end{array}$ & $p=0.4191$ & $R O R A$ & CMC \\
\hline rs7162913 & $\begin{array}{c}\text { rs8038664 } \\
n=2062\end{array}$ & $\begin{array}{ll}\text { GG } & 1011(49.0) \\
\text { GA } & 869(42.1) \\
\text { AA } & 182(8.8)\end{array}$ & $p=0.8597$ & $R O R A$ & CMC \\
\hline $\begin{array}{c}\text { rs9888726 } \\
n=2065\end{array}$ & & $\begin{array}{ll}\text { GG } & 995(48.2) \\
\text { GA } & 890(43.1) \\
\text { AA } & 180(8.7) \\
\end{array}$ & $p=0.1521$ & $R O R A$ & CMC \\
\hline $\begin{array}{c}\mathrm{rs} 9989351 \\
\mathrm{n}=1746\end{array}$ & & $\begin{array}{lr}\text { CC } & 1249(71.5) \\
\text { CA } & 461(26.4)\end{array}$ & $p=0.1946$ & RORA & CMC \\
\hline
\end{tabular}




\begin{tabular}{|c|c|c|c|c|c|}
\hline & & AA $36(2.1)$ & & & \\
\hline rs10869497 & $\begin{array}{l}\text { rs10869495 } \\
\text { n=613 }\end{array}$ & $\begin{array}{ll}\text { GG } & 182(29.7) \\
\text { GA } & 309(50.4) \\
\text { AA } & 122(19.9) \\
\end{array}$ & $p=0.6494$ & $R O R B$ & CMC \\
\hline rs35752149 & $\begin{array}{c}\text { rs1323368 } \\
n=2064\end{array}$ & $\begin{array}{cr}\text { AA } & 1440(69.7) \\
\text { GA } & 573(27.7) \\
\text { GG } & 51(2.5) \\
\end{array}$ & $p=0.2631$ & $R O R B$ & CMC \\
\hline $\begin{array}{c}\text { rs3752955 } \\
\text { n }=2064\end{array}$ & & $\begin{array}{lr}\text { AA } & 671(32.5) \\
\text { AC } & 1040(50.4) \\
\text { CC } & 353(17.1) \\
\end{array}$ & $p=0.8696$ & $R O R B$ & CMC \\
\hline rs73524533 & $\begin{array}{c}\text { rs11143948 } \\
\mathrm{n}=2065\end{array}$ & $\begin{array}{lr}\text { AA } & 1186(57.4) \\
\text { AG } & 740(35.8) \\
\text { GG } & 139(6.7) \\
\end{array}$ & $p=0.2857$ & $R O R B$ & CMC \\
\hline rs2291738 & $\begin{array}{r}\text { rs774047 } \\
n=2063\end{array}$ & $\begin{array}{lr}\text { AA } & 577(27.9) \\
\text { AG } & 1013(49.1) \\
\text { GG } & 473(22.9) \\
\end{array}$ & $p=0.685$ & TIMELESS & GTEX \\
\hline rs17110451 & $\begin{array}{c}\text { rs4131348 } \\
n=1451\end{array}$ & $\begin{array}{cc}\text { GG } & 1060(73.1) \\
\text { GA } & 357(24.6) \\
\text { AA } & 34(2.3) \\
\end{array}$ & $p=0.3225$ & ТPH2 & CMC \\
\hline $\begin{array}{c}\text { rs4760820 } \\
\text { n=1756 }\end{array}$ & & $\begin{array}{ll}\text { CC } & 713(40.6) \\
\text { CA } & 791(45.0) \\
\text { AA } & 252(14.4) \\
\end{array}$ & $p=0.8211$ & ТРН2 & CMC \\
\hline
\end{tabular}


APPENDIX F

UNIVERSITY OF PITTSBURGH INSTITUTIONAL REVIEW BOARD APPROVAL 


\section{University of Pittsburgh Institutional Review Board}

3500 Fifth Avenue Pittsburgh, PA 15213 (412) $383-1480$ (412) 383-1508 (fax) http://www.irb.pitt.edu

\section{Memorandum}

To: Ann Johansson

From: IRB Office

Date: 8/11/2015

IRB\#: $\quad$ PRO15050345

Subject: Variations in Genes Related to Sleep in Children with Autism Spectrum Disorder

The above-referenced protocol has been reviewed by the University of Pittsburgh Institutional Review Board. Based on the information provided to the IRB, this project includes no involvement of human subjects, according to the federal regulations [§45 CFR 46.102(f)]. That is, the investigator conducting research will not obtain information about research subjects via an interaction with them, nor will the investigator obtain identifiable private information. Should that situation change, the investigator must notify the IRB immediately.

Given this determination, you may now begin your project.

Please note the following information:

- If any modifications are made to this project, use the "Send Comments to IRB Staff" process from the project workspace to request a review to ensure it continues to meet the determination.

- Upon completion of your project, be sure to finalize the project by submitting a "Study Completed" report from the project workspace.

Please be advised that your research study may be audited periodically by the University of Pittsburgh Research Conduct and Compliance Office. 
APPENDIX G

\section{LICENSE AGREEMENT FOR MANUSCRIPT \#1}




\section{Springer Publishing Company, Inc. LICENSE}

TERMS AND CONDITIONS

This is a License Agreement between Ann Johansson ("You") and Springer Publishing Company, Inc. ("Springer Publishing Company, Inc.") provided by Copyright Clearance Center ("CCC"). The license consists of your order details, the terms and conditions provided by Springer Publishing Company, Inc, and the payment terms and conditions.

All payments must be made in full to CCC. For payment instructions, please see information listed at the bottom of this form.

\begin{tabular}{|c|c|}
\hline License Number & 4267280160570 \\
\hline License date & Jan 13,2018 \\
\hline Licensed content publisher & Springer Publishing Company, Inc. \\
\hline Licensed content title & Journal of nursing measurement \\
\hline Licensed content date & $\operatorname{Jan} 1,1993$ \\
\hline Type of Use & Thesis/Dissertation \\
\hline Requestor type & Academic institution \\
\hline Format & Electronic \\
\hline Portion & chapter/article \\
\hline $\begin{array}{l}\text { The requesting } \\
\text { person/organization is: }\end{array}$ & University of Pittsburgh \\
\hline $\begin{array}{l}\text { Title or numeric reference of the } \\
\text { portion(s) }\end{array}$ & $\begin{array}{l}\text { Psychometric properties of the Simons Simplex Collection } \\
\text { Sleep Interview }\end{array}$ \\
\hline $\begin{array}{l}\text { Title of the article or chapter the } \\
\text { portion is from }\end{array}$ & $\begin{array}{l}\text { Psychometric properties of the Simons Simplex Collection } \\
\text { Sleep Interview }\end{array}$ \\
\hline E ditor of portion(s) & Janice L Hinkle \\
\hline Author of portion(s) & $\begin{array}{l}\text { Ann Johansson, Jeffrey Rohay, Christine Feeley, Eileen } \\
\text { Chasens }\end{array}$ \\
\hline Volume of serial or monograph. & $\mathrm{N} / \mathrm{A}$ \\
\hline Page range of the portion & \\
\hline Publication date of portion & in press \\
\hline Rights for & Main product \\
\hline Duration of use & Life of current edition \\
\hline Creation of copies for the disabled & no \\
\hline With minor editing privileges & no \\
\hline For distribution to & Worldw ide \\
\hline In the follow ing Ianguage(s) & Original language of publication \\
\hline With incidental promotional use & no \\
\hline $\begin{array}{l}\text { The lifetime unit quantity of new } \\
\text { product }\end{array}$ & Up to 499 \\
\hline Title & Dr \\
\hline Instructor name & Ann Johansson \\
\hline Institution name & other \\
\hline
\end{tabular}


Expected presentation date

Billing Type

Billing Address
Apr 2018

Invoice

Ann Johansson

7520 Penn Ave \# 32

Homew ood, PA 15208

United States

Attn: Ann Johansson

0.00 USD

Total (may include ccc user fee)

Terms and Conditions

\section{TERMS AND CONDITIONS}

The following terms are individual to this publisher:

A maximum of $10 \%$ of the content may be licensed for republication.

User is responsible for identifying and seeking separate licenses (under this service or otherwise) for, any of such third party materials which are identified anywhere in the work by permission; without a separate license, such third party materials may not be used.

\section{Other Terms and Conditions: \\ ST ANDARD TERMS AND CONDITIONS}

1. Description of Service; Defined Terms. This Republication License enables the User to obtain licenses for republication of one or more copyrighted works as described in detail on the relevant Order Confirmation (the "Work(s)"). Copyright Clearance Center, Inc. ("CCC") grants licenses through the Service on behalf of the rightsholder identified on the Order Confimation (the "Rightsholder"). "Republication", as used herein, generally means the inclusion of a Work, in whole or in part, in a new work or works, also as described on the Order Confirmation. "User", as used herein, means the person or entity making such republication.

2. The terms set forth in the relevant Order Confimation, and any terms set by the Rightsholder with respect to a particular Work, govem the terms of use of Works in connection with the Service. By using the Service, the person transacting for a republication license on behalf of the User represents and warrants that he/she/it (a) has been duly authorized by the User to accept, and hereby does accept, all such terms and conditions on behalf of User, and (b) shall inform User of all such terms and conditions. In the event such person is a "freelancer" or other third party independent of User and CCC, such party shall be deemed joirtly a "User" for purposes of these terms and conditions. In any event, User shall be deemed to heve accepted and agreed to all such terms and conditions if User republishes the Work in any fashion.

\section{Scope of License; Limitations and Obligations.}

3.1 All Works and all rights therein, including copyright rights, remain the sole and exclusive property of the Rightsholder. The license created by the exchange of an Order Confimation (and/or any invoice) and payment by User of the full amount set forth on that document includes only those rights expressly set forth in the Order Confimation and in these terms and conditions, and conveys no other rights in the Work(s) to User. All rights not expressly granted are hereby reserved.

3.2 General Payment Terms: You may pay by credit card or through an account with us payable at the end of the month. If you and we agree that you may establish a standing account with CCC, then the following terms apply: Remit Payment to: Copyright Clearance Center, 29118 Network Place, Chicago, $\mathbb{L}$ 60673-1291. Payments Due: Invoices are payable upon their delivery to you (or upon our notice to you that they are available to you for downloading). After 30 days, outstanding amounts will be subject to a service charge of 1-1/2\% per month or, if less, the maximum rate allowed by applicable law. Unless otherwise specifically set forth in the Order Confirmation or in a separate written agreement signed by CCC, invoices are due and payable on "net 30" tems. While User may exercise the rights 
licensed immediately upon issuance of the Order Confirmation, the license is automatically revoked and is null and void, as if it had never been issued, if complete payment for the license is not received on a timely basis either from User directly or through a payment agent, such as a credit card company.

3.3 Unless otherwise provided in the Order Confirmation, any grant of rights to User (i) is

"one-time" (including the editions and product family specified in the license), (ii) is non-

exclusive and non-transferable and (iii) is subject to any and all limitations and restrictions (such as, but not limited to, limitations on duration of use or circulation) included in the Order Confirmation or invoice and/or in these terms and conditions. Upon completion of the licensed use, User shall either secure a new permission for further use of the Work (s) or immediately cease any new use of the Work's) and shall render iraccessible (such as by deleting or by removing or severing links or other locators) any further copies of the Work (except for copies printed on paper in accordance with this license and still in User's stock at the end of such period).

3.4 In the event that the material for which a republication license is sought includes third party materials (such as photographs, illustrations, graphs, inserts and similar materials) which are identified in such material as having been used by permission, User is responsible for identifying, and seeking separate licenses (under this Service or otherwise) for, any of such third party materials; without a separate license, such third party materials may not be used.

3.5 Use of proper copyright notice for a Work is required as a condition of any license granted under the Service. Unless otherwise provided in the Order Confirmation, a proper copyright notice will read substantially as follows: "Republished with permission of [Rightsholder's name], from [Work's title, author, volume, edition number and year of copyright]; permission conveyed through Copyright Clearance Center, Inc. "Such notice must be provided in a reasonably legible font size and must be placed either immediately adjacent to the Work as used (for example, as part of a by-line or footnote but not as a separate electronic link) or in the place where substantially all other credits or notices for the new work containing the republished Work are located. Failure to include the required notice results in loss to the Rightsholder and CCC, and the User shall be liable to pay liquidated damages for each such failure equal to twice the use fee specified in the Order Confimation, in addition to the use fee itself and any other fees and charges specified. 3.6 User may only make alterations to the Work if and as expressly set forth in the Order Confirmation. No Work may be used in any way that is defarmatory, violates the rights of third parties (including such third parties' rights of copyright, privacy, publicity, or other tangible or intangible property), or is otherwise illegal, sexually explicit or obscene. In addition, User may not conjoin a Work with any other material that may result in damage to the reputation of the Rightsholder. User agrees to inform CCC if it becomes aware of any infringement of any rights in a Work and to cooperate with any reasonable request of $\mathrm{CCC}$ or the Rightsholder in connection therewith. 4. Indemity. User hereby indermifies and agrees to defend the Rightsholder and CCC, and their respective employees and directors, against all claims, liability, damages, costs and expenses, including legal fees and expenses, arising out of any use of a Work beyond the scope of the rights granted herein, or any use of a Work which has been altered in any unauthorized way by User, including claims of defamation or infringement of rights of copyright, publicity, privacy or other tangible or intangible property.

5. Limitation of Liability. UNDER NO CIRCUMSTANCES WLL CCC OR THE RIGHTSHOLDER BE LIABLE FOR ANY DIRECT, INDIRECT, CONSEQUENTIAL OR INCIDENTAL DAMAGES (INCLUDING WITHOUT LIMITA TION DAMAGES FOR LOSS OF BUSINESS PROFITS OR INFORMATION, OR FOR BUSINESS INTERRUPTION) ARISING OUT OF THE USE OR INAB ILITY TO USE A WORK, EVEN IF ONE OF THEM HAS BEEN ADVISED OF THE POSSIB ILITY OF SUCH DAMAGES. In any event, the total liability of the Rightsholder and CCC (including their 
respective employees and directors) shall not exceed the total amount actually paid by User for this license. User assumes full liability for the actions and omissions of its principals, employees, agents, affiliates, successors and assigns.

6. Limited Warranties. THE WORK(S) AND RIGHT(S) ARE PROVDED “AS IS". CCC HAS THE RIGHT TO GRANT TO USER THE RIGHTS GRANTED IN THE ORDER CONFIRMA TION DOCUMENT. CCC AND THE RIGHTSHOLDER DISCLAIM ALL OTHER WARRANTIES RELATIING TO THE WORK(S) AND RIGHT(S), EITHER EXPRESS OR IMPLIED, INCLUDING WITHOUT LMITA TION IMPLED WARRANTIES OF MERCHANTAB ILITY OR FITNESS FOR A PARTICULAR PURPOSE. ADDITIONAL RIGHTS MAY BE REQURED TO USE ILLUSTRA TIONS, GRAPHS, PHOTOGRAPHS, AB STRACTS, INSER TS OR OTHER POR TIONS OF THE WORK (AS OPPOSED TO THE ENTIRE WORK) IN A MANNER CONTEMPLA TED B Y USER; USER UNDERSTANDS AND AGREES THA T NEITHER CCC NOR THE RIGHTSHOLDER MAY HAVE SUCH ADDITIONAL RIGHTS TO GRANT.

7. Effect of Breach. Any failure by User to pay any amount when due, or any use by User of a Work beyond the scope of the license set forth in the Order Confirmation and/or these terms and conditions, shall be a material breach of the license created by the Order Confirmation and these terms and conditions. Any breach not cured within 30 days of written notice thereof shall result in immediate termination of such license without further notice. Any unauthorized (but licensable) use of a Work that is terminated immediately upon notice thereof may be liquidated by payment of the Rightsholder's ordinary license price therefor; any unauthorized (and unlicensable) use that is not teminated immediately for any reason (including, for example, because materials containing the Work cannot reasonably be recalled) will be subject to all remedies available at law or in equity, but in no event to a payment of less than three times the Rightsholder's ordinary license price for the most closely analogous licensable use plus Rightsholder's and/or CCC's costs and expenses incurred in collecting such payment.

8. Miscellaneous.

8.1 User acknowledges that CCC may, from time to time, make changes or additions to the Service or to these terms and conditions, and $\mathrm{CCC}$ reserves the right to send notice to the User by electronic mail or otherwise for the purposes of notifying User of such changes or additions, provided that any such changes or additions shall not apply to permissions already secured and paid for. 8.2 Use of User-related information collected through the Service is governed by CCC's privacy policy, available online here:

http://WwWw copvright. com/content/cc3/en/tools/footer/privacwpolicy.html.

8.3 The licensing transaction described in the Order Confirmation is persoral to User. There fore, User may not assign or transfer to any other person (whether a natural person or an organization of any kind) the license created by the Order Confirmation and these terms and conditions or any rights granted hereunder; provided, however, that User may assign such license in its entirety on written notice to $\mathrm{CCC}$ in the event of a transfer of all or substantially all of User's rights in the new material which includes the Work(s) licensed under this Service.

8.4 No amendment or waiver of any terms is binding unless set forth in writing and signed by the parties. The Rightsholder and CCC hereby object to any terms contained in any writing prepared by the User or its principals, employees, agents or affiliates and purporting to govern or otherwise relate to the licensing transaction described in the Order Confirmation, which terms are in any way inconsistent with any terms set forth in the Order Confirmation and/or in these terms and conditions or CCC's standard operating procedures, whether such witing is prepared prior to, simultaneously with or subsequent to the Order Confirmation, and whether such writing appears on a copy of the Order Confirmation or in a separate instrument. 
8.5 The licensing transaction described in the Order Confirmation document shall be governed by and construed under the law of the State of New York, USA, without regard to the principles there of of conflicts of law. Any case, controversy, suit, action, or proceeding arising out of, in connection with, or related to such licensing transaction shall be brought, at CCC's sole discretion, in any federal or state court located in the County of New York, State of New York, USA, or in any federal or state court whose geographical jurisdiction covers the location of the Rightsholder set forth in the Order Confirmation. The parties expressly submit to the personal jurisdiction and venue of each such federal or state court.If you have any comments or questions about the Service or Copyright Clearance Center, please contact us at 978-750-8400 or send an e-mail to info@copyright.com.

v 1.1

Questions? customercare@conviahtcom or +1-855-239-3415 (toll free in the US) or $+1-978-646-2777$. 
APPENDIX H

MANUSCRIPT 2 SUPPLEMENTARY INFORMATION 


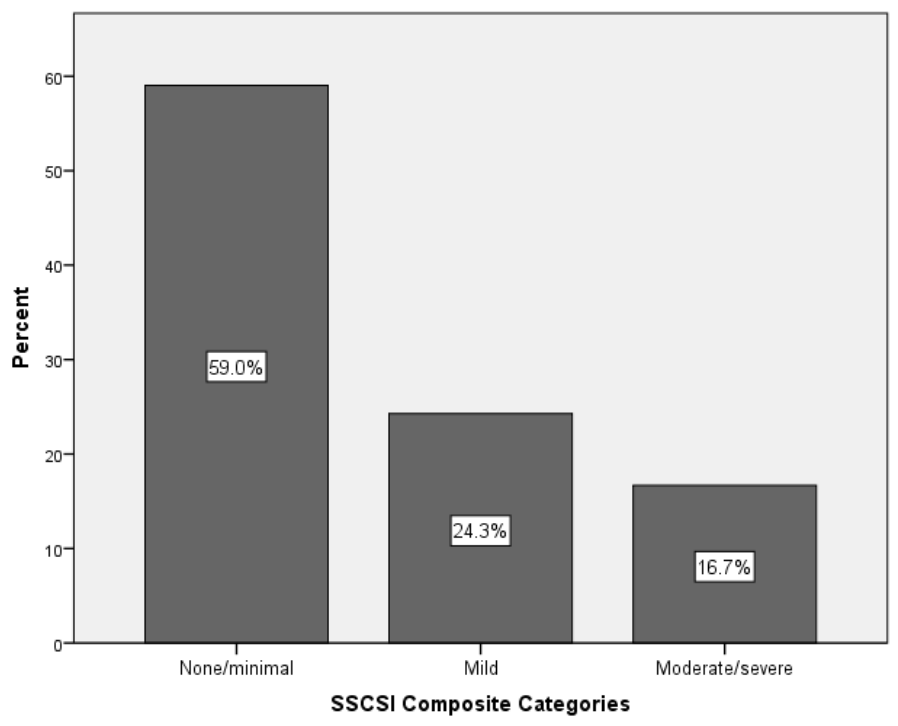

Figure 11: Distribution of SSCSI Composite Sleep Score Categories 
Table 15: Sample characteristics by SSCSI Sleep Score Categories

\begin{tabular}{|c|c|c|c|c|}
\hline & $\begin{array}{c}\text { Full Sample } \\
\underline{N=2,619}\end{array}$ & $\begin{array}{c}\text { None/minimal } \\
\underline{n=1429}\end{array}$ & $\begin{array}{c}\text { Mild } \\
\underline{\mathrm{n}=684}\end{array}$ & $\begin{array}{c}\text { Moderate/severe } \\
\underline{n=506}\end{array}$ \\
\hline Age (yrs.) [mean(SD)] & $9.0(3.6)^{\mathrm{a}}$ & $9.2(3.6)^{b, d}$ & $8.8(3.5)$ & $8.7(3.5)$ \\
\hline Male & $2,272(86.8)^{a}$ & $1,270(88.9)^{b, d}$ & $571(83.5)$ & $431(85.2)$ \\
\hline $\begin{array}{l}\text { ADOS severity score } \\
\text { [mean(SD)] }\end{array}$ & $7.4(1.7)$ & $7.4(1.7)$ & $7.6(1.6)$ & $7.4(1.6)$ \\
\hline $\begin{array}{l}\text { SA severity score } \\
\text { [mean(SD)] }\end{array}$ & $7.2(1.7)$ & $7.2(1.8)$ & $7.4(1.7)$ & $7.2(1.7)$ \\
\hline $\begin{array}{l}\text { RRB severity score } \\
\text { [mean(SD)] }\end{array}$ & $7.8(1.8)$ & $7.8(1.9)$ & $7.9(1.8)$ & $7.8(1.7)$ \\
\hline \multicolumn{5}{|l|}{ Diagnosis: } \\
\hline ASD & $274(10.5)$ & $173(12.1)^{b}$ & $63(9.2)$ & $53(10.5)$ \\
\hline AD & 2,345 (89.5) & $1,256(87.9)$ & $621(90.8)$ & 453 (89.5) \\
\hline $\mathrm{NVIQ} \leq 70$ & $695(26.6)^{a}$ & $355(24.8)^{d}$ & $182(26.6)$ & $158(31.2)$ \\
\hline Seizures & $135(5.2)^{\mathrm{a}}$ & $61(4.3)^{d}$ & $38(5.6)$ & $36(7.1)$ \\
\hline GI disturbance & $1,307(49.9)^{\mathrm{a}}$ & $640(44.8)^{b, d}$ & $359(52.5)$ & $308(60.9)^{c}$ \\
\hline $\begin{array}{l}\text { Regular sleep duration } \\
\text { (hours) [mean(SD)] }\end{array}$ & $9.2(1.3)^{a}$ & $9.5(1.1)^{b, d}$ & $9.2(1.3)$ & $8.3(1.6)^{c}$ \\
\hline $\begin{array}{l}\text { Duration less than NSF } \\
\text { recommendations }\end{array}$ & $741(28.3)^{a}$ & $297(19.3)^{b, d}$ & $214(34.4)$ & $230(53.9)^{c}$ \\
\hline
\end{tabular}

Note: data presented as n (\%) unless otherwise specified; ADOS=Autism Diagnostic Observation Scale, range 4-10; $A S D=$ autism spectrum disorder (ADOS score 4-5); $A D=$ autism disorder (ADOS score 6-10); NVIQ=nonverbal IQ; NSF=National Sleep Foundation; RRB=restricted, repetitive behavior; SA=social affect; ${ }^{\text {a} D i f f e r e n c e ~ b e t w e e n ~ n o n e / m i n i m a l, ~ m i l d, ~ a n d ~ m o d e r a t e / s e v e r e, ~ K r u s k a l-~}$ Wallis, $p<0.05$; ${ }^{\text {b }}$ Difference between none/minimal and mild, $p<0.05$; ${ }^{\mathrm{c}}$ Difference between mild and moderate/severe, $p<0.05$; ${ }^{\mathrm{d}}$ Difference between none/minimal and moderate/severe, $p<0.05$ 


\section{Table 16: Reported SSCSI Item Frequencies by Sleep Score Categories}

\begin{tabular}{|c|c|c|c|}
\hline & $\begin{array}{l}\text { Total Sample } \\
\qquad \mathbf{N = 2 , 6 1 9}\end{array}$ & $\begin{array}{l}\text { Mild } \\
\underline{n}=684\end{array}$ & $\begin{array}{c}\text { Moderate/severe } \\
\underline{n=506}\end{array}$ \\
\hline \multicolumn{4}{|l|}{ Nighttime problems } \\
\hline Bedtime problems & 466 (17.8) & $135(19.8)$ & 318 (62.8) \\
\hline $\begin{array}{l}\text { Need to lay down with } \\
\text { child }\end{array}$ & $500(19.1)$ & $173(25.4)$ & $283(56.3)$ \\
\hline Difficulty falling asleep & $833(31.8)$ & $325(47.6)$ & $420(83.7)$ \\
\hline $\begin{array}{l}\text { Frequent/prolonged } \\
\text { awakenings }\end{array}$ & $492(18.8)$ & $172(25.4)$ & $264(52.5)$ \\
\hline \multicolumn{4}{|l|}{ Daytime problems } \\
\hline Difficulty waking & 351 (13.4) & $104(15.2)$ & 222 (43.9) \\
\hline $\begin{array}{l}\text { Excess daytime } \\
\text { sleepiness }\end{array}$ & 351 (13.4) & $116(17.1)$ & $196(39.0)$ \\
\hline Long/ frequent naps & $100(3.8)$ & $31(4.5)$ & $64(12.7)$ \\
\hline $\begin{array}{l}\text { Regular duration is not } \\
\text { enough }\end{array}$ & $560(21.4)$ & $184(28.0)$ & $289(60.6)$ \\
\hline \multicolumn{4}{|l|}{$\begin{array}{l}\text { Sleep duration } \\
\text { problems }\end{array}$} \\
\hline $\begin{array}{l}\text { Irregular bedtime and/or } \\
\quad \text { waketime * }\end{array}$ & 513 (19.6) & $130(23.6)$ & $202(47.1)$ \\
\hline No regular duration & 149 (5.7) & $37(5.4)$ & $90(18.0)$ \\
\hline
\end{tabular}


Table 17: SSCSI Composite and Subscale Frequencies by Sleep Score Categories

\begin{tabular}{|c|c|c|c|}
\hline & $\begin{array}{c}\text { Full } \\
\text { Sample } \\
\mathbf{N = 2 , 6 1 9} \\
\end{array}$ & $\begin{array}{c}\text { Mild } \\
\mathbf{n}=684\end{array}$ & $\begin{array}{c}\text { Moderate/ } \\
\text { severe } \\
\text { n=506 }\end{array}$ \\
\hline \multicolumn{4}{|l|}{$\begin{array}{l}\text { Children with sleep } \\
\text { problems * }\end{array}$} \\
\hline Composite & $1,073(41.0)$ & & \\
\hline Nighttime problems & $675(25.8)$ & $238(34.8)$ & 437 (86.4) \\
\hline Daytime problems & $348(13.3)$ & $89(13.0)$ & 259 (51.2) \\
\hline $\begin{array}{l}\text { Sleep duration } \\
\text { problems }\end{array}$ & $72(2.7)$ & $8(1.2)$ & $64(12.6)$ \\
\hline
\end{tabular}


Table 18: Associations Between Child Characteristics and SSCSI Sleep Problems in Early

Childhood

\begin{tabular}{|c|c|c|c|c|}
\hline & \multicolumn{3}{|c|}{$\begin{array}{c}\text { None/minimal vs. } \\
\text { Mild-severe }\end{array}$} \\
\hline & & OR & CI & $p$ \\
\hline \multirow{8}{*}{$\begin{array}{l}\text { SSCSI } \\
\text { composite } \\
\text { sleep score }\end{array}$} & Age & 0.84 & $\begin{array}{l}0.63- \\
1.12\end{array}$ & 0.23 \\
\hline & Sex * & 1.25 & $\begin{array}{l}0,92- \\
1.71\end{array}$ & 0.15 \\
\hline & NVIQ & 0.82 & $\begin{array}{l}0.74- \\
0.90\end{array}$ & $\begin{array}{l}<\mathbf{0 . 0} \\
\mathbf{1}\end{array}$ \\
\hline & ADOS & 1.03 & $\begin{array}{l}0.96- \\
1.09\end{array}$ & 0.41 \\
\hline & SA ${ }^{t}$ & - & - & - \\
\hline & $\mathbf{R R B}^{\mathrm{t}}$ & - & - & - \\
\hline & GID & 1.61 & $\begin{array}{l}1.31- \\
1.99\end{array}$ & $\begin{array}{l}<0.0 \\
1\end{array}$ \\
\hline & Seizures & 1.19 & $\begin{array}{l}0.71- \\
2.01\end{array}$ & 0.28 \\
\hline
\end{tabular}

\section{Subscales}

\begin{tabular}{|c|c|c|c|c|}
\hline \multirow{8}{*}{$\begin{array}{l}\text { Nighttime } \\
\text { problems }\end{array}$} & Age & 0.94 & $\begin{array}{l}0.86- \\
1.01\end{array}$ & 0.10 \\
\hline & Sex * & 1.05 & $\begin{array}{l}0.75- \\
1.48\end{array}$ & 0.76 \\
\hline & NVIQ & 0.80 & $\begin{array}{l}\text { 0.72- } \\
0.89\end{array}$ & $\begin{array}{l}<\mathbf{0 . 0} \\
\mathbf{1}\end{array}$ \\
\hline & ADOS & 0.98 & $\begin{array}{l}0.91- \\
1.05\end{array}$ & 0.60 \\
\hline & $\mathbf{S A}^{\mathrm{t}}$ & - & - & - \\
\hline & $\mathbf{R R B}^{\mathrm{t}}$ & - & - & - \\
\hline & GID & 1.37 & $\begin{array}{l}1.09- \\
1.73\end{array}$ & $\begin{array}{l}<\mathbf{0 . 0} \\
\mathbf{1}\end{array}$ \\
\hline & Seizures & 1.46 & $\begin{array}{l}0.85- \\
2.50 \\
\end{array}$ & 0.17 \\
\hline \multirow{4}{*}{$\begin{array}{l}\text { Daytime } \\
\text { problems }\end{array}$} & Age & 0.95 & $\begin{array}{l}0.66- \\
1.38\end{array}$ & 0.80 \\
\hline & Sex $*$ & 1.59 & $\begin{array}{l}1.05- \\
2.37\end{array}$ & 0.02 \\
\hline & NVIQ & 1.01 & $\begin{array}{l}0.87- \\
1.16\end{array}$ & 0.92 \\
\hline & ADOS & 1.03 & $\begin{array}{l}0.94- \\
1.13\end{array}$ & 0.56 \\
\hline
\end{tabular}




\begin{tabular}{|c|c|c|c|c|}
\hline & $\mathbf{S A}^{\mathrm{t}}$ & - & - & - \\
\hline & $\mathbf{R R B}^{\mathrm{t}}$ & - & - & - \\
\hline & GID & 2.19 & $\begin{array}{l}1.60- \\
3.04\end{array}$ & $\begin{array}{l}<0.0 \\
1\end{array}$ \\
\hline & Seizures & 0.89 & $\begin{array}{l}0.41- \\
1.91\end{array}$ & 0.76 \\
\hline \multirow{8}{*}{$\begin{array}{l}\text { Sleep } \\
\text { duration } \\
\text { problems }\end{array}$} & Age & 0.67 & $\begin{array}{l}0.42- \\
1.09\end{array}$ & 0.11 \\
\hline & Sex * & 1.23 & $\begin{array}{l}0.54- \\
2.79\end{array}$ & 0.62 \\
\hline & NVIQ & 0.65 & $\begin{array}{l}\text { 0.52- } \\
0.82\end{array}$ & $\begin{array}{l}<0.0 \\
1\end{array}$ \\
\hline & ADOS & 1.01 & $\begin{array}{l}0.84- \\
1.21 \\
\end{array}$ & 0.93 \\
\hline & $\mathbf{S A}^{\mathrm{t}}$ & - & - & - \\
\hline & $\mathbf{R R B}^{\mathrm{t}}$ & - & - & - \\
\hline & GID & 1.01 & $\begin{array}{l}0.56- \\
1.84\end{array}$ & 0.97 \\
\hline & Seizures & 1.74 & $\begin{array}{l}0.52- \\
5.80\end{array}$ & 0.37 \\
\hline
\end{tabular}

Bold results denote statistical significance; * Reference group=females; ${ }^{\mathrm{t}}$ Only tested if ADOS severity score significant, ADOS=ADOS severity score; CI=95\% confidence interval; GID=gastrointestinal disturbance; NVIQ=non-verbal IQ; OR=odds ratio; $\mathrm{SA}=\mathrm{ADOS}$ social affect severity score; $\mathrm{RRB}=\mathrm{ADOS}$ restricted, repetitive behavior severity score 
Table 19: Associations Between Child Characteristics and SSCSI Sleep Problems in Middle Childhood

\begin{tabular}{|c|c|c|c|c|}
\hline & \multicolumn{3}{|c|}{$\begin{array}{c}\text { None/minimal vs. } \\
\text { Mild-severe }\end{array}$} \\
\hline & & OR & CI & $p$ \\
\hline \multirow{8}{*}{$\begin{array}{l}\text { SSCSI } \\
\text { composite } \\
\text { sleep score }\end{array}$} & Age & 1.15 & $\begin{array}{l}1.04- \\
1.27\end{array}$ & $\begin{array}{l}<0.0 \\
1\end{array}$ \\
\hline & Sex * & 1.86 & $\begin{array}{l}1.23- \\
2.83\end{array}$ & $\begin{array}{l}<\mathbf{0 . 0} \\
1\end{array}$ \\
\hline & NVIQ & 1.07 & $\begin{array}{l}0.95- \\
1.19 \\
\end{array}$ & 0.27 \\
\hline & ADOS & 1.01 & $\begin{array}{l}0.94- \\
1.10\end{array}$ & 0.72 \\
\hline & $\mathbf{S A}^{\mathrm{t}}$ & - & - & - \\
\hline & $\mathbf{R R B}^{\mathrm{t}}$ & - & - & - \\
\hline & GID & 1.56 & $\begin{array}{l}1.18- \\
2.06\end{array}$ & $\begin{array}{l}<0.0 \\
1\end{array}$ \\
\hline & Seizures & 1.74 & $\begin{array}{l}0.97- \\
3.22\end{array}$ & 0.07 \\
\hline
\end{tabular}

\section{Subscales}

\begin{tabular}{|c|c|c|c|c|}
\hline \multirow{8}{*}{$\begin{array}{l}\text { Nighttime } \\
\text { problems }\end{array}$} & Age & 0.80 & $\begin{array}{l}0.55- \\
1.17\end{array}$ & 0.25 \\
\hline & Sex * & 1.46 & $\begin{array}{l}0.93- \\
2.26\end{array}$ & 0.09 \\
\hline & NVIQ & 0.96 & $\begin{array}{l}0.85- \\
1.09\end{array}$ & 0.54 \\
\hline & ADOS & 1.05 & $\begin{array}{l}0.96- \\
1.15\end{array}$ & 0.29 \\
\hline & $\mathbf{S A}^{\mathrm{t}}$ & - & - & - \\
\hline & $\mathbf{R R B}^{\mathrm{t}}$ & - & - & - \\
\hline & GID & 1.63 & $\begin{array}{l}1.19- \\
2.24\end{array}$ & $\begin{array}{l}<\mathbf{0 . 0} \\
\mathbf{1}\end{array}$ \\
\hline & Seizures & 1.60 & $\begin{array}{l}0.86- \\
2.88\end{array}$ & 0.13 \\
\hline \multirow{4}{*}{$\begin{array}{l}\text { Daytime } \\
\text { problems }\end{array}$} & Age & 1.18 & $\begin{array}{l}1.03- \\
1.36\end{array}$ & 0.02 \\
\hline & Sex $*$ & 1.31 & $\begin{array}{l}0.75- \\
2.28\end{array}$ & 0.35 \\
\hline & NVIQ & 1.11 & $\begin{array}{l}0.94- \\
1.31\end{array}$ & 0.22 \\
\hline & ADOS & 0.97 & $\begin{array}{l}0.86- \\
1.09\end{array}$ & 0.61 \\
\hline
\end{tabular}




\begin{tabular}{|c|c|c|c|c|}
\hline & $\mathbf{S A}^{\mathrm{t}}$ & - & - & - \\
\hline & $\mathbf{R R B}^{\mathrm{t}}$ & - & - & - \\
\hline & GID & 1.98 & $\begin{array}{l}1.32- \\
3.01\end{array}$ & $\begin{array}{l}<\mathbf{0 . 0} \\
1\end{array}$ \\
\hline & Seizures & 1.72 & $\begin{array}{l}0.83- \\
3.35\end{array}$ & 0.12 \\
\hline \multirow{8}{*}{$\begin{array}{l}\text { Sleep } \\
\text { duration } \\
\text { problems }\end{array}$} & Age & 0.80 & $\begin{array}{l}0.33- \\
1.93\end{array}$ & 0.61 \\
\hline & Sex * & 1.60 & $\begin{array}{l}0.53- \\
4.86\end{array}$ & 0.40 \\
\hline & NVIQ & 0.73 & $\begin{array}{l}0.54- \\
0.99\end{array}$ & 0.04 \\
\hline & ADOS & 1.03 & $\begin{array}{l}0.80- \\
1.32\end{array}$ & 0.84 \\
\hline & $\mathbf{S A}^{\mathrm{t}}$ & - & - & - \\
\hline & $\mathbf{R R B}^{\mathrm{t}}$ & - & - & - \\
\hline & GID & 1.44 & $\begin{array}{l}0.60- \\
3.46\end{array}$ & 0.42 \\
\hline & Seizures & 3.00 & $\begin{array}{l}0.81- \\
8.82\end{array}$ & 0.06 \\
\hline
\end{tabular}

Bold results denote statistical significance; * Reference group=females; ${ }^{\mathrm{t}}$ Only tested if ADOS severity score significant, ADOS=ADOS severity score; $\mathrm{CI}=95 \%$ confidence interval; GID=gastrointestinal disturbance; NVIQ=non-verbal IQ; OR=odds ratio; SA=ADOS social affect severity score; $\mathrm{RRB}=\mathrm{ADOS}$ restricted, repetitive behavior severity score 
Table 20: Associations Between Child Characteristics and SSCSI Sleep Problems in Late Childhood

\begin{tabular}{|l|l|lll|}
\hline \multicolumn{2}{|c|}{} & \multicolumn{3}{|c|}{$\begin{array}{c}\text { None/minimal vs. } \\
\text { Mild-severe }\end{array}$} \\
\cline { 3 - 5 } \multicolumn{1}{|c|}{} & Age & 1.17 & $\begin{array}{l}0.86- \\
1.59\end{array}$ & 0.31 \\
\hline \multirow{4}{*}{$\begin{array}{l}\text { SSCSI } \\
\text { composite } \\
\text { sleep score }\end{array}$} & Sex ${ }^{*}$ & 1.70 & $\begin{array}{l}0.90- \\
3.19\end{array}$ & 0.10 \\
\cline { 2 - 5 } & \multirow{4}{*}{ NVIQ } & 1.06 & $\begin{array}{l}0.87- \\
1.28\end{array}$ & 0.57 \\
\cline { 2 - 5 } & ADOS & 1.06 & $\begin{array}{l}0.92- \\
1.23\end{array}$ & 0.40 \\
\cline { 2 - 5 } & SA & - & - & - \\
\cline { 2 - 5 } & RRB & - & - & - \\
\cline { 2 - 5 } & \multirow{4}{*}{ GID } & 1.23 & $\begin{array}{l}0.76- \\
1.97\end{array}$ & 0.39 \\
\cline { 2 - 5 } & \multirow{2}{*}{ Seizures } & 0.94 & $\begin{array}{l}0.38- \\
2.33\end{array}$ & 0.89 \\
\hline
\end{tabular}

Subscales

\begin{tabular}{|c|l|lll|}
\hline \multirow{4}{*}{} & Age & 1.23 & $\begin{array}{l}0.87- \\
1.74\end{array}$ & 0.25 \\
\cline { 2 - 5 } & Sex ${ }^{*}$ & 0.87 & $\begin{array}{l}0.38- \\
2.00\end{array}$ & 0.74 \\
\cline { 2 - 5 } $\begin{array}{c}\text { Nighttime } \\
\text { problems }\end{array}$ & NVIQ & 0.99 & $\begin{array}{l}0.78- \\
1.25\end{array}$ & 0.94 \\
\cline { 2 - 5 } & ADOS & 0.98 & $\begin{array}{l}0.82- \\
1.16\end{array}$ & 0.78 \\
\cline { 2 - 5 } & SA $^{\mathrm{t}}$ & - & - & - \\
\cline { 2 - 5 } & RRB & - & - & - \\
\cline { 2 - 5 } & GID & $\mathbf{2 . 0 8}$ & $\begin{array}{l}\mathbf{1 . 1 5 -} \\
\mathbf{3 . 8 5}\end{array}$ & $\mathbf{0 . 0 2}$ \\
\cline { 2 - 5 } & Seizures & 1.11 & $\begin{array}{l}0.39- \\
3.18\end{array}$ & 0.85 \\
\hline \multirow{4}{*}{$\begin{array}{c}\text { Daytime } \\
\text { problems }\end{array}$} & Age & 1.14 & $\begin{array}{l}0.78- \\
1.66\end{array}$ & 0.50 \\
\cline { 2 - 5 } & Sex $*$ & 2.23 & $\begin{array}{l}0.96- \\
4.90\end{array}$ & 0.05 \\
\cline { 2 - 5 } & NVIQ & 1.36 & $\begin{array}{l}1.01- \\
1.90\end{array}$ & 0.06 \\
\hline
\end{tabular}




\begin{tabular}{|c|c|c|c|c|}
\hline & ADOS & 0.97 & $\begin{array}{l}0.79- \\
1.18\end{array}$ & 0.73 \\
\hline & $\mathbf{S A}^{\mathrm{t}}$ & - & - & - \\
\hline & $\mathbf{R R B}^{\mathrm{t}}$ & - & - & - \\
\hline & GID & 1.07 & $\begin{array}{l}0.55- \\
2.07\end{array}$ & 0.85 \\
\hline & Seizures & 0.91 & $\begin{array}{l}0.25- \\
3.30\end{array}$ & 0.89 \\
\hline \multirow{8}{*}{$\begin{array}{l}\text { Sleep } \\
\text { duration } \\
\text { problems }\end{array}$} & Age & 1.09 & $\begin{array}{l}0.06- \\
20.42\end{array}$ & 0.95 \\
\hline & Sex $*$ & 1.14 & $\begin{array}{l}0.13- \\
10.18\end{array}$ & 0.91 \\
\hline & NVIQ & 0.98 & $\begin{array}{l}0.51- \\
1.90\end{array}$ & 0.96 \\
\hline & ADOS & 0.87 & $\begin{array}{l}0.53- \\
1.42 \\
\end{array}$ & 0.57 \\
\hline & $\mathbf{S A}^{\mathrm{t}}$ & - & - & - \\
\hline & $\mathbf{R R B}^{\mathrm{t}}$ & - & - & - \\
\hline & GID & 0.92 & $\begin{array}{l}0.18- \\
4.75\end{array}$ & 0.92 \\
\hline & Seizures & $\begin{array}{l}<0.0 \\
0\end{array}$ & 0 & - \\
\hline
\end{tabular}

Bold results denote statistical significance; * Reference group=females; ${ }^{\mathrm{t}}$ Only tested if ADOS severity score significant, ADOS=ADOS severity score; $\mathrm{CI}=95 \%$ confidence interval; GID=gastrointestinal disturbance; NVIQ=non-verbal IQ; OR=odds ratio; SA=ADOS social affect severity score; $\mathrm{RRB}=\mathrm{ADOS}$ restricted, repetitive behavior severity score 
APPENDIX I

TRANSMISSION DISEQUILIBRIUM TEST RESULTS 
Table 21: Transmission Disequilibrium Test Results

\begin{tabular}{|c|c|c|c|c|c|c|c|c|c|}
\hline & Variant & Gene & $\begin{array}{c}\text { Reference } \\
\text { allele }\end{array}$ & b & OR & $\begin{array}{c}95 \% \\
\text { CI } \\
\text { lower }\end{array}$ & $\begin{array}{c}95 \% \\
\text { CI } \\
\text { upper }\end{array}$ & $\begin{array}{c}\text { Trios } \\
\text { (n) }\end{array}$ & $\begin{array}{c}p \text { - } \\
\text { value* }\end{array}$ \\
\hline \multirow{3}{*}{$\begin{array}{l}\text { Composite } \\
\text { sleep problems }\end{array}$} & rs28730724 & CIART & A & 0.487 & 1.63 & 1.11 & 2.38 & 110 & 0.012 \\
\hline & rs4775351 & RORA & A & 0.180 & 1.20 & 1.01 & 1.42 & 461 & 0.038 \\
\hline & rs135721 & CSNK1E & A & 0.161 & 1.17 & 1.01 & 1.37 & 508 & 0.041 \\
\hline $\begin{array}{l}\text { Nighttime } \\
\text { problems }\end{array}$ & rs6431588 & PER2 & A & 0.311 & 1.36 & 1.09 & 1.71 & 262 & 0.007 \\
\hline \multirow{6}{*}{$\begin{array}{l}\text { Daytime } \\
\text { problems }\end{array}$} & rs11598 & MTNR1B & G & 0.440 & 1.55 & 1.08 & 2.24 & 113 & 0.019 \\
\hline & rs4296527 & CSNK1E & A & 0.212 & 1.24 & 1.03 & 1.49 & 354 & 0.023 \\
\hline & rs11943456 & CLOCK & G & 0.204 & 1.23 & 1.05 & 1.43 & 506 & 0.009 \\
\hline & rs4775351 & $R O R A$ & A & 0.203 & 1.23 & 1.01 & 1.48 & 362 & 0.037 \\
\hline & rs13395562 & $G A D 1$ & G & 0.192 & 1.21 & 1.03 & 1.42 & 477 & 0.018 \\
\hline & rs693367 & CLOCK & A & 0.194 & 1.21 & 1.03 & 1.43 & 453 & 0.019 \\
\hline
\end{tabular}

Note: Results with $p<0.05$ and trios with $\mathrm{n}>100$ are displayed. No trios with $\mathrm{n}>100$ for sleep duration problems subscale. * No significant results after Bonferroni correction $(p<0.0007)$. OR $=$ odds ratio 


\section{BIBLIOGRAPHY}

Achenbach, T. (2001). Child Behavior Checklist for Ages 6-18, 1-4.

Agnati, L. F., Barlow, P. W., Baluška, F., Tonin, P., Guescini, M., Leo, G., \& Fuxe, K. (2011). A new theoretical approach to the functional meaning of sleep and dreaming in humans based on the maintenance of "predictive psychic homeostasis." Communicative \& Integrative Biology, 4(6), 640654. http://doi.org/10.4161/cib.4.6.17602

Akshoomoff, N. (2006). Use of the Mullen Scales of Early Learning for the assessment of young children with autism spectrum disorders. Child Neuropsychology, 12(4-5), 269-277. http://doi.org/10.1080/09297040500473714

Al-Sahab, B., Ardern, C. I., Hamadeh, M. J., \& Tamim, H. (2012). Age at menarche and current substance use among Canadian adolescent girls: Results of a cross-sectional study. BMC Public Health, 12(1), 195. http://doi.org/10.1186/1471-2458-12-195

Alam, M. N., Kumar, S., Bashir, T., Suntsova, N., Methippara, M. M., Szymusiak, R., \& McGinty, D. (2005). GABA-mediated control of hypocretin- but not melanin-concentrating hormoneimmunoreactive neurones during sleep in rats. The Journal of Physiology, 563(2), 569-582. http://doi.org/10.1113/jphysiol.2004.076927

Aldinger, K. A., Lane, C. J., Veenstra-VanderWeele, J., \& Levitt, P. (2015). Patterns of risk for multiple co-occurring medical conditions replicate across distinct cohorts of children with autism spectrum disorder. Autism Research, 8, 771-781. http://doi.org/10.1002/aur.1492

Allebrandt, K. V, Teder-Laving, M., Akyol, M., Pichler, I., Müller-Myhsok, B., Pramstaller, P., ... Roenneberg, T. (2010). CLOCK gene variants associate with sleep duration in two independent populations. Biological Psychiatry, 67(11), 1040-7. http://doi.org/10.1016/j.biopsych.2009.12.026

Allik, H., Larsson, J. O., \& Smedje, H. (2008). Sleep patterns in school-age children with Asperger syndrome or high-functioning autism: A follow-up study. Journal of Autism and Developmental Disorders, 38(9), 1625-33. http://doi.org/10.1007/s10803-008-0543-0

Ambrosius, U., Lietzenmaier, S., Wehrle, R., Wichniak, A., Kalus, S., Winkelmann, J., ... Friess, E. (2008). Heritability of sleep electroencephalogram. Biological Psychiatry, 64(4), 344-8. http://doi.org/10.1016/j.biopsych.2008.03.002

American Academy of Sleep Medicine. (2014). International Classification of Sleep Disorders. (3rd ed.). Darien, IL: American Academy of Sleep Medicine.

American Psychiatric Association. (2013). Autism spectrum disorder. In Diagnostic and statistical 
manual of mental disorders (DSM-5). Arlington, VA: American Psychiatric Association.

Anaclet, C., Parmentier, R., Ouk, K., Guidon, G., Buda, C., Sastre, J. P., ... Lin, J. S. (2009). Orexin/hypocretin and histamine: Distinct roles in the control of wakefulness demonstrated using knock-out mouse models. Journal of Neuroscience, 29(46), 14423-14438. http://doi.org/10.1523/JNEUROSCI.2604-09.2009

Ancoli-Israel, S., Cole, R., Alessi, C., Chamers, M., Moorcroft, W., \& Pollak, C. (2003). The role of actigraphy in the study of sleep and circadian rhythms. Sleep, 26(3), 342-392.

André, E., Conquet, F., Steinmayr, M., Stratton, S. C., Porciatti, V., \& Becker-André, M. (1998). Disruption of retinoid-related orphan receptor beta changes circadian behavior, causes retinal degeneration and leads to vacillans phenotype in mice. The EMBO Journal, 17(14), 3867-77. http://doi.org/10.1093/emboj/17.14.3867

Andretic, R., van Swinderen, B., \& Greenspan, R. J. (2005). Dopaminergic modulation of arousal in Drosophila. Current Biologyy, 15(13), 1165-75. http://doi.org/10.1016/j.cub.2005.05.025

Archer, S. N., Carpen, J. D., Gibson, M., Lim, G. H., Johnston, J. D., Skene, D. J., \& von Schantz, M. (2010). Polymorphism in the PER3 promoter associates with diurnal preference and delayed sleep phase disorder. Sleep, 33(5), 695-701.

Archer, S. N., Robilliard, D. L., Skene, D. J., Smits, M., Williams, A., \& Arendt, J. (2003). A length polymorphism in the circadian clock gene Per3 is linked to delayed sleep phase syndrome and extreme diurnal preference. Sleep, 26(4), 24-26.

Auton, A., Abecasis, G. R., Altshuler, D. M., Durbin, R. M., Abecasis, G. R., Bentley, D. R., ... Abecasis, G. R. (2015). A global reference for human genetic variation. Nature, 526(7571), 68-74. http://doi.org/10.1038/nature15393

Axelrod, B. N. (2002). Validity of the Wechsler Abbreviated Scale of Intelligence and other very short forms of estimating intellectual functioning. Assessment, 9(1), 17-23. http://doi.org/10.1177/1073191102009001003

Bamshad, M., Song, C. K., \& Bartness, T. J. (1999). CNS origins of the sympathetic nervous system outflow to brown adipose tissue. American Journal of Physiology, 276, R1569-1578.

Barmada, M. M. (2016). Family-based association methods. Pittsburgh, PA: Graduate School of Public Health, University of Pittsburgh.

Basheer, R., Porkka-Heiskanen, T., Strecker, R. E., Thakkar, M. M., \& McCarley, R. W. (2000). Adenosine as a biological signal mediating sleepiness following prolonged wakefulness. Biological Signals and Receptors, 9(6), 319-27. http://doi.org/14655

Bishop, S. L., Guthrie, W., Coffing, M., \& Lord, C. (2011). Convergent validity of the Mullen Scales of Early Learning and the Differential Ability Scales in children with autism spectrum disorders. American Journal on Intellectual and Developmental Disabilities, 116(5), 331-43. http://doi.org/10.1352/1944-7558-116.5.331

Bjorness, T. E., Kelly, C. L., Gao, T., Poffenberger, V., \& Greene, R. W. (2009). Control and function of the homeostatic sleep response by adenosine A1 receptors. Journal of Neuroscience, 29(5), 1267- 
1276. http://doi.org/10.1523/JNEUROSCI.2942-08.2009

Blomeke, B., Golka, K., Griefahn, B., \& Roemer, H. C. (2008). Arylalkylamine N-acetyltransferase (AANAT) genotype as a personal trait in melatonin synthesis. Journal of Toxicology and Environmental Health. Part A, 71(13-14), 874-6. http://doi.org/10.1080/15287390801988020

Bolte, S., Dziobek, I., \& Poustka, F. (2009). Brief report: The level and nature of autistic intelligence revisited. Journal of Autism \& Developmental Disorders, 39, 678-682. http://doi.org/10.1007/s10803-008-0667-2

Borbély, A. A., Daan, S., Wirz-Justice, A., \& Deboer, T. (2016). The two-process model of sleep regulation: A reappraisal. Journal of Sleep Research, (September 2014), 131-143. http://doi.org/10.1111/jsr.12371

Born, J., Kellner, C., Uthgenannt, D., Kern, W., \& Fehm, H. L. (1992). Vasopressin regulates human sleep by reducing rapid-eye-movement sleep. The American Journal of Physiology, 262(3 Pt 1), E295-300.

Bradley-Johnson, S. (1997). Test reviews: Mullen Scales of Early Learning. Psychology in the Schools, 34(4), 379-382.

Brennan, K., Bates, E., Shapiro, R., Zyuzin, J., Hallows, W., Huang, Y., ... Jones, C. (2013). Casein kinase 1-delta mutations in familial migraine and advanced sleep phase. Scientific Translational Medicine, 5, 183RA56.

Buckley, A., Rodriguez, A., Jennison, K., Buckley, J., Thurm, A., Sato, S., \& Swedo, S. (2010). REM sleep percentage in children in autism compared with developmental delay and typical develoment. Archives of Pediatrics, 164(11), 1032-1037. http://doi.org/10.1001/archpediatrics.2010.202.REM

Buckley, A., Wingert, K., Swedo, S., Thurm, A., Sato, S., Appel, S., \& Rodriguez, A. J. (2013). First night effect analysis in a cohort of young children with autism spectrum disorder. Journal of Clinical Sleep Medicine, 9(1), 67-70. http://doi.org/10.5664/jcsm.2344

Buijs, R. M., Chun, S. J., Niijima, A., Romijn, H. J., \& Nagai, K. (2001). Parasympathetic and sympathetic control of the pancreas: A role for the suprachiasmatic nucleus and other hypothalamic centers that are involved in the regulation of food intake. Journal of Comparative Neurology, 431, 405-423.

Buijs, R. M., Wortel, J., Van Heerikhuize, J. J., Feenstra, M. G. P., Ter Horst, G. J., Romijn, H. J., \& Kalsbeek, A. (1999). Anatomical and functional demonstration of a multisynaptic suprachiasmatic nucleus adrenal (cortex) pathway. European Journal of Neuroscience, 11, 1535-1544.

Cabrera, S. M., Bright, G. M., Frane, J. W., Blethen, S. L., \& Lee, P. A. (2014). Age of thelarche and menarche in contemporary US females: A cross-sectional analysis. Journal of Pediatric Endocrinology and Metabolism, 27(0), 47-51. http://doi.org/10.1016/j.biotechadv.2011.08.021

Cain, N., \& Gradisar, M. (2010). Electronic media use and sleep in school-aged children and adolescents: A review. Sleep Medicine, 11(8), 735-742. http://doi.org/10.1016/j.sleep.2010.02.006

Canivez, G. L., Konold, T. R., Collins, J. M., \& Wilson, G. (2009). Construct validity of the Wechsler Abbreviated Scale of Intelligence and Wide Range Intelligence Test: Convergent and structural 
validity. School Psychology Quarterly, 24(4), 252-265. http://doi.org/10.1037/a0018030

Carkaci-Salli, N., Flanagan, J. M., Martz, M. K., Salli, U., Walther, D. J., Bader, M., \& Vrana, K. E. (2006). Functional domains of human tryptophan hydroxylase 2 (hTPH2). The Journal of Biological Chemistry, 281(38), 28105-12. http://doi.org/10.1074/jbc.M602817200

Carney, C. E., Buysse, D. J., Ancoli-Israel, S., Edinger, J. D., Krystal, A. D., Lichstein, K. L., \& Morin, C. M. (2012). The Consensus Sleep Diary: Standardizing prospective sleep self-monitoring. Sleep, 35(2), 287-302. http://doi.org/10.5665/sleep.1642

Carpen, J. D., von Schantz, M., Smits, M., Skene, D. J., \& Archer, S. N. (2006). A silent polymorphism in the PER1 gene associates with extreme diurnal preference in humans. Journal of Human Genetics, 51(12), 1122-5. http://doi.org/10.1007/s10038-006-0060-y

Carskadon, M. A. (2002). Factors influencing sleep patterns of adolescents. In M. A. Carskadon (Ed.), Adolescent sleep patterns: Biological, social, and psychological influences (pp. 4-26). Cambridge, UK: Cambridge University Press.

Carskadon, M. A., \& Dement, W. C. (2017). Normal human sleep. In M. Kryger \& T. Roth (Eds.), Principles and Practice of Sleep Medicine (6th ed., pp. 15-24). St. Louis, MO: Elsevier. http://doi.org/10.1016/B978-0-323-24288-2.00002-7

Carskadon, M., Harvey, K., Duke, P., Anders, T., Litt, I., \& Dement, W. (1979). Pubertal changes in daytime sleepiness. Sleep, 2(4), 453-460.

Carter, P. A. (2005). Bereaved caregivers' descriptions of sleep: Impact on daily life and the bereavement process. Oncology Nursing Forum, 32(4), 70-75.

Centers for Disease Control and Prevention. (2016). Community report from the Autism and Developmental Disabilities Monitoring (ADDM) Network: A snapshot of autism spectrum disorder among 8-year-old children in multiple communities across the United States in 2012. Retrieved from http://www.cdc.gov/ncbddd/autism/states/comm_report_autism_2014.pdf

Chaste, P., Clement, N., Botros, H. G., Guillaume, J.-L., Konyukh, M., Pagan, C., ... Bourgeron, T. (2011). Genetic variations of the melatonin pathway in patients with attention-deficit and hyperactivity disorders. Journal of Pineal Research, 51(4), 394-399. http://doi.org/10.1111/j.1600079X.2011.00902.x

Chaste, P., Clement, N., Mercati, O., Guillaume, J.-L., Delorme, R., Botros, H. G., ... Bourgeron, T. (2010). Identification of pathway-biased and deleterious melatonin receptor mutants in autism spectrum disorders and in the general population. PLoS ONE, 5(7), e11495. http://doi.org/10.1371/journal.pone.0011495

Cheng, M. Y., Bullock, C. M., Li, C., Lee, A. G., Bermak, J. C., Belluzzi, J., ... Zhou, Q.-Y. (2002). Prokineticin 2 transmits the behavioural circadian rhythm of the suprachiasmatic nucleus. Nature, 417(6887), 405-10. http://doi.org/10.1038/417405a

Clinton, J. M., Davis, C. J., Zielinski, M. R., Jewett, K. A., \& Krueger, J. M. (2011). Biochemical regulation of sleep and sleep biomarkers. Journal of Clinical Sleep Medicine, 7(5 Suppl), S38-42. http://doi.org/10.5664/JCSM.1360 
Coon, S. L. C., Azuruk, K. M., Ernard, M. B., Oseboom, P. H. R., Lein, D. C. K., \& Odriguez, I. R. R. (1996). The human serotonin N-acetyltransferase (EC 2.3.1.87) gene (AANAT): Structure, chromosomal localization, and tissue expression. Genomics, 84(34), 76-84.

Cortesi, F., Giannotti, F., Ivanenko, A., \& Johnson, K. (2010). Sleep in children with autistic spectrum disorder. Sleep Medicine, 11(7), 659-64. http://doi.org/10.1016/j.sleep.2010.01.010

Cortesi, F., Giannotti, F., Sebastiani, T., Panunzi, S., \& Valente, D. (2012). Controlled-release melatonin, singly and combined with cognitive behavioural therapy, for persistent insomnia in children with autism spectrum disorders: a randomized placebo-controlled trial. Journal of Sleep Research, 21(6), 700-9. http://doi.org/10.1111/j.1365-2869.2012.01021.x

Cotton, S., \& Richdale, A. (2006). Brief report: Parental descriptions of sleep problems in children with autism, Down syndrome, and Prader-Willi syndrome. Research in Developmental Disabilities, 27(2), 151-61. http://doi.org/10.1016/j.ridd.2004.12.003

Crowley, S., Tarokh, L., \& Carskadon, M. (2014). Sleep during adolescence. In S. Sheldon, R. Ferber, M. H. Kryger, \& D. Gozal (Eds.), Principles and Practice of Pediatric Sleep Medicine (2nd ed., pp. 4551). St. Louis, MO: Elsevier Inc.

Cuomo, B. M., Vaz, S., Lee, E. A. L., Thompson, C., Rogerson, J. M., \& Falkmer, T. (2017). Effectiveness of Sleep-Based Interventions for Children with Autism Spectrum Disorder: A MetaSynthesis. Pharmacotherapy, 37(5), 555-578. http://doi.org/10.1002/phar.1920

Dahl, R. E., \& Lewin, D. S. (2002). Pathways to adolescent health sleep regulation and behavior. The Journal of Adolescent Health, 31(6 Suppl), 175-84. Retrieved from http://www.ncbi.nlm.nih.gov/pubmed/12470913

Dardente, H., \& Cermakian, N. (2007). Molecular circadian rhythms in central and peripheral clocks in mammals. Chronobiology International, 24(2), 195-213. http://doi.org/10.1080/07420520701283693

Darko, D. F., Miller, J. C., Gallen, C., White, J., Koziol, J., Brown, S. J., ... Mitler, M. M. (1995). Sleep electroencephalogram delta-frequency amplitude, night plasma levels of tumor necrosis factor alpha, and human immunodeficiency virus infection. Proceedings of the National Academy of Sciences of the United States of America, 92(26), 12080-12084.

Davidson, J., Goin-Kochel, R. P., Green-Snyder, L. A., Hundley, R. J., Warren, Z., \& Peters, S. U. (2014). Expression of the broad autism phenotype in simplex autism families from the Simons Simplex Collection. Journal of Autism and Developmental Disorders, 44(10), 2392-2399. http://doi.org/10.1007/s10803-012-1492-1

De Gennaro, L., Marzano, C., Fratello, F., Moroni, F., Pellicciari, M. C., Ferlazzo, F., ... Rossini, P. M. (2008). The electroencephalographic fingerprint of sleep is genetically determined: A twin study. Annals of Neurology, 64(4), 455-60. http://doi.org/10.1002/ana.21434

Desachy, G., Croen, L. A., Torres, A. R., Kharrazi, M., Delorenze, G. N., Windham, G. C., ... Weiss, L. A. (2015). Increased female autosomal burden of rare copy number variants in human populations and in autism families. Molecular Psychiatry, 20(2), 170-5. http://doi.org/10.1038/mp.2014.179

DeVincent, C. J., Gadow, K. D., Delosh, D., \& Geller, L. (2007). Sleep disturbance and its relation to 
DSM-IV psychiatric symptoms in preschool-age children with pervasive developmental disorder and community controls. Journal of Child Neurology, 22(2), 161-9. http://doi.org/10.1177/0883073807300310

Diekelmann, S., Wilhelm, I., Wagner, U., \& Born, J. (2013). Sleep to implement an intention. Sleep, 36(1), 149-153.

Dubocovich, M. L., Benloucif, S., \& Masana, M. I. (1996). Melatonin receptors in the mammalian suprachiasmatic nucleus. Behavioural Brain Research, 73(1-2), 141-7.

Dumont, R., Cruse, C., Alfonso, V., \& Levine, C. (2000). Mullen Scales of Early Learning: AGS Edition. Journal of Psychoeducational Assessment, 18(4), 381-389.

Ebrahim, I. O., Howard, R. S., \& Kopelman, M. D. (2002). The hypocretin/orexin system. Journal of the Royal Society of Medicine, 95(5), 227-230. http://doi.org/10.1258/jrsm.95.5.227

Eicher, J. D., Landowski, C., Stackhouse, B., Sloan, A., Chen, W., Jensen, N., ... Johnson, A. D. (2015). GRASP v2.0: an update on the Genome-Wide Repository of Associations between SNPs and Phenotypes. Nucleic Acids Research, 43(D1), D799-D804. http://doi.org/10.1093/nar/gku1202

Elia, M., Ferri, R., Musumeci, S. A., Del Gracco, S., Bottitta, M., Scuderi, C., ... Grubar, J. C. (2000). Sleep in subjects with autistic disorder: A neurophysiological and psychological study. Brain \& Development, 22(2), 88-92.

Elliott, C. D. (2012). The Differential Ability Scales-Second Edition. In D. P. Flanagan \& P. L. Harrison (Eds.), Contemporary Intellectual Assessment: Theories, Tests, and Issues. (3rd ed., pp. 336-356). New York: The Guilford Press.

Elrod, M. G., \& Hood, B. S. (2015). Sleep differences among children with autism spectrum disorders and typically developing peers: A meta-analysis. Journal of Developmental and Behavioral Pediatrics, 36(3), 166-177. http://doi.org/10.1097/DBP.0000000000000140

Erlander, M. G., Tillakaratne, N. J. K., Feldblum, S., Patel, N., \& Tobin, a J. (1991). Two genes encode distinct gluamate decarboxylases. Neuron, 7, 91-100.

Etain, B., Jamain, S., Milhiet, V., Lajnef, M., Boudebesse, C., Dumaine, A., ... Bellivier, F. (2014). Association between circadian genes, bipolar disorders and chronotypes. Chronobiology International, 31(7), 807-14. http://doi.org/10.3109/07420528.2014.906445

Evans, D. S., Parimi, N., Nievergelt, C. M., Blackwell, T., Redline, S., Ancoli-Israel, S., ... Tranah, G. J. (2013). Common genetic variants in ARNTL and NPAS2 and at chromosome 12p13 are associated with objectively measured sleep traits in the elderly. Sleep, 36(3), 431-46. http://doi.org/10.5665/sleep.2466

Fadini, C. C., Lamônica, D. A., Fett-conte, A. C., Osório, E., Zuculo, G. M., Giacheti, C. M., \& Pinato, L. (2015). Influence of sleep disorders on the behavior of individuals with autism spectrum disorder. Frontiers in Human Neuroscience, 9, 1-8. http://doi.org/10.3389/fnhum.2015.00347

Fairfax, B. P., Humburg, P., Makino, S., Naranbhai, V., Wong, D., Lau, E., ... Mcgee, C. (2014). Innate immune activity conditions the effect of regulatory variants upon monocyte gene expression. Science, 343(6175), 1-30. http://doi.org/10.1126/science.1246949.Innate 
Fass, R., Fullerton, S., Tung, S., \& Mayer, E. A. (2000). Sleep disturbances in clinic patients with functional bowel disorders. The American Journal of Gastroenterology, 95(5), 1195-1200.

Fischbach, G. D., \& Lord, C. (2010). The Simons Simplex Collection: A resource for identification of autism genetic risk factors. Neuron, 68(2), 192-5. http://doi.org/10.1016/j.neuron.2010.10.006

Fletcher, F. E., Foster-Owens, M. D., Conduit, R., Rinehart, N. J., Riby, D. M., \& Cornish, K. M. (2016). The development trajectory of parent-report and objective sleep profiles in autism spectrum disorder: Associations with anxiety and bedtime routines. Autism, 21(4), 493-503. http://doi.org/10.1177/1362361316653365

Frank, M. G., Stryker, M. P., \& Tecott, L. H. (2002). Sleep and sleep homeostasis in mice lacking the 5HT2c receptor. Neuropsychopharmacology, 27(5), 869-73. http://doi.org/10.1016/S0893133X(02)00353-6

Franken, P., Lopez-Molina, L., Marcacci, L., Schibler, U., \& Tafti, M. (2000). The transcription factor DBP affects circadian sleep consolidation and rhythmic EEG activity. The Journal of Neuroscience, 20(2), 617-25.

Frazier, T. W., Georgiades, S., Bishop, S. L., \& Hardan, A. Y. (2014). Behavioral and cognitive characteristics of females and males with autism in the Simons Simplex Collection. Journal of the American Academy of Child and Adolescent Psychiatry, 53(3), 329-340.e3. http://doi.org/10.1016/j.jaac.2013.12.004

Gail Williams, P., Sears, L. L., \& Allard, A. (2004). Sleep problems in children with autism. Journal of Sleep Research, 13, 265-268.

Galli-Carminati, G., Deriaz, N., \& Bertschy, G. (2009). Melatonin in treatment of chronic sleep disorders in adults with autism: A retrospective study. Swiss Medical Weekly, 139(19-20), 293-6. http://doi.org/smw-12342

Gamsiz, E. D., Viscidi, E. W., Frederick, A. M., Nagpal, S., Sanders, S. J., Murtha, M. T., ... Morrow, E. M. (2013). Intellectual disability is associated with increased runs of homozygosity in simplex autism. American Journal of Human Genetics, 93(1), 103-9. http://doi.org/10.1016/j.ajhg.2013.06.004

Gerendai, I., Toth, I. E., Boldogkoi, Z., Medveczky, I., \& Halasz, B. (2000). CNS structures presumably involved in vagal control of ovarian function. Journal of the Autonomic Nervous System, 80, 40-45.

Giallo, R., Wood, C. E., Jellett, R., \& Porter, R. (2013). Fatigue, wellbeing and parental self-efficacy in mothers of children with an autism spectrum disorder. Autism, 17(4), 465-80. http://doi.org/10.1177/1362361311416830

Giannotti, F., Cortesi, F., Cerquiglini, A., \& Bernabei, P. (2006). An open-label study of controlledrelease melatonin in treatment of sleep disorders in children with autism. Journal of Autism and Developmental Disorders, 36(6), 741-752. http://doi.org/10.1007/s10803-006-0116-z

Giannotti, F., Cortesi, F., Cerquiglini, A., Miraglia, D., Vagnoni, C., Sebastiani, T., \& Bernabei, P. (2008). An investigation of sleep characteristics, EEG abnormalities and epilepsy in developmentally regressed and non-regressed children with autism. Journal of Autism and Developmental Disorders, 38(10), 1888-97. http://doi.org/10.1007/s10803-008-0584-4 
Giannotti, F., Cortesi, F., Cerquiglini, A., Vagnoni, C., \& Valente, D. (2011). Sleep in children with autism with and without autistic regression. Journal of Sleep Research, 20(2), 338-47. http://doi.org/10.1111/j.1365-2869.2010.00882.x

Gibbs, J. R., Van Der Brug, M. P., Hernandez, D. G., Traynor, B. J., Nalls, M. A., Lai, S., ... Singleton, A. B. (2010). Abundant quantitative trait loci exist for DNA methylation and gene expression in human brain. PLoS Genetics, 6(5), e1000952. http://doi.org/10.1371/journal.pgen.1000952

Goel, N., Banks, S., Lin, L., Mignot, E., \& Dinges, D. F. (2011). Catechol-O-methyltransferase Val158Met polymorphism associates with individual differences in sleep physiologic responses to chronic sleep loss. PLoS ONE, 6(12), e29283. http://doi.org/10.1371/journal.pone.0029283

Goldman, S. E., Alder, M. L., Burgess, H. J., Corbett, B. A., Hundley, R., Wofford, D., ... Malow, B. A. (2017). Characterizing sleep in adolescents and adults with autism spectrum disorders. Journal of Autism and Developmental Disorders, 47(6), 1682-1695. http://doi.org/10.1007/s10803-017-3089-1

Goldman, S. E., Bichell, T. J., Surdyka, K., \& Malow, B. A. (2012). Sleep in children and adolescents with Angelman syndrome: Association with parent sleep and stress. Journal of Intellectual Disability Research, 56, 600-608. http://doi.org/10.1111/j.1365-2788.2011.01499.x

Goldman, S. E., Surdyka, K., Cuevas, R., Adkins, K., Wang, L., \& Malow, B. A. (2010). Defining the sleep phenotype in children with autism. Developmental Neuropsychology, 34(5), 560-573. http://doi.org/10.1080/87565640903133509

Goldstein, G., \& Mazefsky, C. (2013). Wechsler Scales of Intelligence. In F. Volkmar (Ed.), Encyclopedia of Autism Spectrum Disorders (pp. 1-17). New York: Springer. http://doi.org/10.1007/978-1-4419-1698-3

Goodlin-Jones, B. L., Sitnick, S. L., Tang, K., Liu, J., \& Anders, T. F. (2008). The Children’s Sleep Habits Questionnaire in toddlers and preschool children. Journal of Developmental and Behavioral Pediatrics, 29, 82-88. http://doi.org/10.1097/DBP.0b013e318163c39a

Goodlin-Jones, B. L., Tang, K., Liu, J., \& Anders, T. F. (2008). Sleep patterns in preschool-age children with autism, developmental delay, and typical development. Journal of the American Academy of Child \& Adolescent Psychiatry, 47(8), 930-938. http://doi.org/10.1097/CHI.0b013e3181799f7c

Goriki, A., Hatanaka, F., Myung, J., Kim, J. K., Yoritaka, T., Tanoue, S., ... Takumi, T. (2014). A novel protein, CHRONO, functions as a core component of the mammalian circadian clock. PLoS Biology, 12(4), e1001839. http://doi.org/10.1371/journal.pbio.1001839

Gorrindo, P., Williams, K. C., Lee, E. B., Walker, L. S., Mcgrew, S. G., \& Levitt, P. (2012). Gastrointestinal dysfunction in autism: Parental report, clinical evaluation, and associated factors. Autism Research, 5, 101-108. http://doi.org/10.1002/aur.237

Gotham, K., Pickles, A., \& Lord, C. (2009). Standardizing ADOS scores for a measure of severity in autism. Journal of Autism and Developmental Disorders, 39(5), 693-705. http://doi.org/10.1007/s10803-008-0674-3

Gotham, K., Risi, S., Dawson, G., Tager-Flusberg, H., Joseph, R., Carter, A., ... Lord, C. (2008). A replication of the Autism Diagnostic Observation Schedule (ADOS) revised algorithms. Journal of the American Academy of Child and Adolescent Psychiatry, 47(6), 642-51. 
http://doi.org/10.1097/CHI.0b013e31816bffb7

Goto, M., Mizuno, M., Matsumoto, A., Yang, Z., \& Jimbo, E. F. (2017). Role of a circadian-relevant gene NR1D1 in brain development: Possible involvement in the pathophysiology of autism spectrum disorders. Nature, (January), 1-12. http://doi.org/10.1038/srep43945

Gottlieb, D. J., O’Connor, G. T., \& Wilk, J. B. (2007). Genome-wide association of sleep and circadian phenotypes. BMC Medical Genetics, 8 Suppl 1, S9. http://doi.org/10.1186/1471-2350-8-S1-S9

Goutagny, R., Luppi, P.-H., Salvert, D., Gervasoni, D., \& Fort, P. (2005). GABAergic control of hypothalamic melanin-concentrating hormone-containing neurons across the sleep-waking cycle. Neuroreport, 16(10), 1069-73. Retrieved from http://www.ncbi.nlm.nih.gov/pubmed/15973150

Green, L., Debra, S., Qixuan, D. A., Raphael, C., Arianne, R. P. G., Wallace, S., ... Ledbetter, D. H. (2016). Autism spectrum disorder, developmental and psychiatric features in 16p11.2 duplication. Journal of Autism \& Developmental Disorders, 46, 2734-2748. http://doi.org/10.1007/s10803-0162807-4

Gringras, P., Nir, T., Breddy, J., Frydman-Marom, A., \& Findling, R. L. (2017). Efficacy and Safety of Pediatric Prolonged-Release Melatonin for Insomnia in Children With Autism Spectrum Disorder. Journal of the American Academy of Child and Adolescent Psychiatry, 56(11), 948-957.e4. http://doi.org/10.1016/j.jaac.2017.09.414

Grundberg, E., Adoue, V., Kwan, T., Ge, B., Duan, Q. L., Lam, K. C. L., ... Pastinen, T. (2011). Global analysis of the impact of environmental perturbation on cis-regulation of gene expression. PloS Genetics, 7(1), e1001279. http://doi.org/10.1371/journal.pgen.1001279

Hamet, P., \& Tremblay, J. (2006). Genetics of the sleep-wake cycle and its disorders. Metabolism: Clinical and Experimental, 55(10 Suppl 2), S7-12. http://doi.org/10.1016/j.metabol.2006.07.006

Hao, K., Bosse, Y., Nickle, D. C., Pare, P. D., Postma, D. S., Laviolette, M., ... Sin, D. D. (2012). Lung eQTLs to help reveal the molecular underpinnings of asthma. PLoS Genetics, 8(11), e1003029. http://doi.org/10.1371/journal.pgen.1003029

He, Y., Jones, C. R., Fujiki, N., Xu, Y., Guo, B., Holder, J. L., ... Fu, Y. (2009). The transcriptional repressor DEC2 regulates sleep length in mammals. Science, 325(5942), 866-870. http://doi.org/10.1126/science.1174443

Hirata, I., Mohri, I., Kato-nishimura, K., \& Tachibana, M. (2016). Sleep problems are more frequent and associated with problematic behaviors in preschoolers with autism spectrum disorder. Research in Developmental Disabilities, 49-50, 86-99. http://doi.org/10.1016/j.ridd.2015.11.002

Hirshkowitz, M., Whiton, K., Albert, S. M., Alessi, C., Bruni, O., DonCarlos, L., ... Adams Hillard, P. J. (2015). National Sleep Foundation's sleep time duration recommendations: Methodology and results summary. Sleep Health, 1(1), 40-43. http://doi.org/10.1016/j.sleh.2014.12.010

Hobson, J. A. (1989). The development of sleep. In Sleep (pp. 71-92). New York: Scientific American Library.

Hodge, D., Carollo, T. M., Lewin, M., Hoffman, C. D., \& Sweeney, D. P. (2014). Sleep patterns in children with and without autism spectrum disorders: Developmental comparisons. Research in 
Developmental Disabilities, 35(7), 1631-1638. http://doi.org/10.1016/j.ridd.2014.03.037

Hohjoh, H., Takasu, M., Shishikura, K., Takahashi, Y., Honda, Y., \& Tokunaga, K. (2003). Significant association of the arylalkylamine $\mathrm{N}$-acetyltransferase ( AA-NAT) gene with delayed sleep phase syndrome. Neurogenetics, 4(3), 151-153. http://doi.org/10.1007/s10048-002-0141-9

Hu, V. W., Sarachana, T., Sherrard, R. M., \& Kocher, K. M. (2015). Investigation of sex differences in the expression of RORA and its transcriptional targets in the brain as a potential contributor to the sex bias in autism. Molecular Autism, 6(7), 1-18.

Huafang, Y., Donohue, S. J., Klein, D. C., \& Mcbride, O. W. (1992). Localization of the hydroxyindoleO-methyltranferase gene to the pseudoautosomal region: Implications for mapping of psychiatric disorders. Human Molecular Genetics, 2(2), 127-131.

Hus, V., Gotham, K., \& Lord, C. (2014). Standardizing ADOS domain scores: Separating severity of social affect and restricted and repetitive behaviors. Journal of Autism \& Developmental Disorders, 44(10), 2400-2412. http://doi.org/10.1007/s10803-012-1719-1

Ibrahim, S. H., Voigt, R. G., Katusic, S. K., Weaver, A. L., \& William, J. (2009). Incidence of gastrointestinal symptoms in children with autism: A population-based study. Pediatrics, 124(2), 680-686. http://doi.org/10.1542/peds.2008-2933

Iglowstein, I., Jenni, O. G., Molinari, L., \& Largo, R. H. (2003). Sleep duration from infancy to adolescence: Reference values and generational trends. Pediatrics, 111(2), 302-307. http://doi.org/10.1542/peds.111.2.302

Isobe, Y., \& Nishino, H. (2004). Signal transmission from the suprachiasmatic nucleus to the pineal gland via the paraventricular nucleus: Analysed from arg-vasopressin peptide, rPer2 mRNA and AVP mRNA changes and pineal AA-NAT mRNA after the melatonin injection during light and dark per. Brain Research, 1013(2), 204-11. http://doi.org/10.1016/j.brainres.2004.04.052

Jackman, A. R., Biggs, S. N., Walter, L. M., Embuldeniya, U. S., Davey, M. J., Nixon, G. M., ... Horne, R. S. C. (2013). Sleep disordered breathing in early childhood: Quality of life for children and families. Sleep, 36(11), 1639-1646. http://doi.org/10.5665/sleep.3116

Jenni, O. G., Achermann, P., \& Carskadon, M. A. (2005). Homeostatic sleep regulation in adolescents. Sleep, 28(11), 1446-1454. Retrieved from http://www.journalsleep.org

Johansson, A. E. E., Feeley, C. A., Dorman, J. S., \& Chasens, E. R. (n.d.). Characteristics of sleep in children with autism spectrum disorders from the Simons Simplex Collection. In Preparation.

Johansson, A. E. E., Rohay, J. M., \& Chasens, E. R. (n.d.). Psychometric properties of the Simons Simplex Collection Sleep Interview. Journal of Nursing Measurement.

Johnson, C. R., Turner, K. S., Foldes, E., Malow, B. A., \& Wiggs, L. (2013). Comparison of sleep questionnaires in the assessment of sleep disturbances in children with autism spectrum disorders. Sleep Medicine, 13(7), 795-801. http://doi.org/10.1016/j.sleep.2012.03.005

Johnson, N. L., \& Simpson, P. M. (2013). Lack of father involvement in research on children with autism spectrum disorder: Maternal parenting stress and family functioning. Issues in Mental Health Nursing, 34(4), 220-8. http://doi.org/10.3109/01612840.2012.745177 
Joseph, R. M., Tager-Flusberg, H., \& Lord, C. (2002). Cognitive profiles and social-communicative functioning in children with autism spectrum disorder. Journal of Child Psychology and Psychiatry, 43(6), 807-821.

Kalsbeek, A., Fliers, E., Franke, A. N., Wortel, J., \& Buijs, R. M. (2000). Functional connections between the suprachiasmatic nucleus and the thyroid gland as revealed by lesioning and viral tracing techniques in the rat. Endocrinology, 141, 3832-3841.

Kawamoto, T., Noshiro, M., Sato, F., Maemura, K., Takeda, N., Nagai, R., ... Kato, Y. (2004). A novel autofeedback loop of Dec1 transcription involved in circadian rhythm regulation. Biochemical and Biophysical Research Communications, 313(1), 117-24. http://doi.org/10.1016/j.bbrc.2003.11.099

Khor, A., Gray, K., Reid, S., \& Melvin, G. (2014). Feasibility and validity of ecological momentary assessment in adolescents with high-functiong autism and Asperger's disorder. Journal of Adolescence, 37(1), 37-46. http://doi.org/10.1016/j.adolescence.2013.10.005

King, D. P., Zhao, Y., Sangoram, A. M., Wilsbacher, L. D., Tanaka, M., Antoch, M. P., ... Takahashi, J. S. (1997). Positional cloning of the mouse circadian clock gene. Cell, 89(4), 641-53. Retrieved from http://www.pubmedcentral.nih.gov/articlerender.fcgi?artid=3815553\&tool=pmcentrez\&rendertype= abstract

Ko, C. H., \& Takahashi, J. S. (2006). Molecular components of the mammalian circadian clock. Human Molecular Genetics, 15(Review Issue 2), R271-R277. http://doi.org/10.1093/hmg/ddl207

Kondratov, R. V, Chernov, M. V, Kondratova, A. A., Gorbacheva, V. Y., Gudkov, A. V, \& Antoch, M. P. (2003). BMAL1-dependent circadian oscillation of nuclear CLOCK: Posttranslational events induced by dimerization of transcriptional activators of the mammalian clock system. Genes \& Development, 17, 1921-1932. http://doi.org/10.1101/gad.1099503

Koopmann, T. T., Adriaens, M. E., Moerland, P. D., Marsman, R. F., Westerveld, M. L., Lal, S., ... Bezzina, C. R. (2014). Genome-wide identification of expression quantitative trait loci (eQTLs) in human heart. PLoS One, 9(5), e97380. http://doi.org/10.1371/journal.pone.0097380

Koskenvuo, M., Hublin, C., Partinen, M., Heikkila, K., \& Kaprio, J. (2007). Heritability of diurnal type: A nationwide study of 8753 adult twin pairs. Journal of Sleep Research, 16(2), 156-162.

Krakowiak, P., Goodlin-Jones, B., Hertz-Picciotto, I., Croen, L. A., \& Hansen, R. L. (2008). Sleep problems in children with autism spectrum disorders, developmental delays, and typical development: A population-based study. Journal of Sleep Research, 17(2), 197-206. http://doi.org/10.1111/j.1365-2869.2008.00650.x

Kripke, D. F., Klimecki, W. T., Nievergelt, C. M., Rex, K. M., Murray, S. S., Shekhtman, T., ... Kelsoe, J. R. (2014). Circadian polymorphisms in night owls, in bipolars, and in non-24-hour sleep cycles. Psychiatry Investig, 11(4), 345-362. http://doi.org/10.4306/pi.2014.11.4.345

Krishna, J. (2014). Polysomnography and MSLT. In S. Sheldon, R. Ferber, M. Kryger, \& D. Gozal (Eds.), Principles and Practice of Pediatric Sleep Medicine (2nd ed., pp. 399-409). St. Louis, MO: Elsevier Inc.

Krueger, J. M., Rector, D. M., \& Churchill, L. (2007). Sleep and cytokines. Sleep Medicine Clinics, 2(2), 161-169.

Retrieved

from 
http://www.pubmedcentral.nih.gov/articlerender.fcgi?artid=2605347\&tool=pmcentrez\&rendertype= abstract

Kulman, G., Lissoni, P., Rovelli, F., Roselli, M. G., Brivio, F., \& Sequeri, P. (2000). Evidence of pineal endocrine hypofunction in autistic children. Neuroendocrinology Letters, 21(1), 31-34. Retrieved from http://www.ncbi.nlm.nih.gov/pubmed/11455326

Kume, K., Kume, S., Park, S. K., Hirsh, J., \& Jackson, F. R. (2005). Dopamine is a regulator of arousal in the fruit fly. The Journal of Neuroscience, 25(32), 7377-84. http://doi.org/10.1523/JNEUROSCI.2048-05.2005

Kushida, C., Littner, M., \& Morgenthaler, T. (2005). Practice parameters for the indications for polysomnography and related procedures: An update for 2005. Sleep, 28(4), 499-521.

Laird, N., \& Lange, C. (2011). Association analysis in family designs. In The Fundamentals of Modern Statistical Genetics (pp. 139-142). New York: Springer Science+Business Media.

Lambert, A., Tessier, S., Rochette, A.-C., Scherzer, P., Mottron, L., \& Godbout, R. (2016). Poor sleep affects daytime functioning in typically developing and autistic children not complaining of sleep problems: A questionnaire-based and polysomnographic study. Research in Autism Spectrum Disorders, 23, 94-106. http://doi.org/10.1016/j.rasd.2015.11.010

Lamontagne, S., Singh, R., \& Palosky, C. (2010). Daily Media Use Among Children and Teens Up Dramatically From Five Years Ago. Washington, D.C. Retrieved from http://kff.org/disparitiespolicy/press-release/daily-media-use-among-children-and-teens-up-dramatically-from-five-yearsago/

Lappalainen, T., Sammeth, M., Friedländer, M. R., 't Hoen, P. A. C., Monlong, J., Rivas, M. A., ... Dermitzakis, E. T. (2013). Transcriptome and genome sequencing uncovers functional variation in humans. Nature, 501(7468), 506-511. http://doi.org/10.1038/nature12531

Lauritsen, M. B., Børglum, A. D., Betancur, C., Philippe, A., Kruse, T. A., Leboyer, M., \& Ewald, H. (2002). Investigation of two variants in the DOPA decarboxylase gene in patients with autism. American Journal of Medical Genetics, 114(4), 466-70. http://doi.org/10.1002/ajmg.10379

Leu, R. M., Beyderman, L., Botzolakis, E. J., Surdyka, K., Wang, L., \& Malow, B. (2011). Relation of melatonin to sleep architecture in children with autism. Journal of Autism and Developmental Disorders, 41(4), 427-433. http://doi.org/10.1007/s10803-010-1072-1

Lewandowski, A. S., Toliver-Sokol, M., \& Palermo, T. M. (2011). Evidence-based review of subjective pediatric sleep measures. Journal of Pediatric Psychology, 36(7), 780-793. http://doi.org/10.1093/jpepsy/jsq119

Li, J., Hu, W., Boehmer, L., Cheng, M., Lee, A., Jilek, A., ... Zhou, Q. (2006). Attenuated circadian rhythms in mice lacking the Prokineticin 2 gene. Journal of Neuroscience, 26(45), 11615-11623. http://doi.org/10.1021/ja8019214.Optimization

Li, Q., Stram, A., Chen, C., Kar, S., Gayther, S., Haiman, C., ... Freedman, M. L. (2014). Expression QTL-based analyses reveal candidate causal genes and loci across five tumor types. Human Molecular Genetics, 23(19), 5294-5302. http://doi.org/10.1093/hmg/ddu228 
Licis, A. (2018). Sleep disorders: Assessment and treatment in preschool-aged children. Child and Adolescent Psychiatry Clinics of North America, 26(3), 587-595. http://doi.org/10.1016/j.chc.2017.02.009

Limoges, É., Bolduc, C., Berthiaume, C., Mottron, L., \& Godbout, R. (2013). Relationship between poor sleep and daytime cognitive performance in young adults with autism. Research in Developmental Disabilities, 34(4), 1322-1335. http://doi.org/10.1016/j.ridd.2013.01.013

Liu, C., \& Reppert, S. M. (2000). GABA synchronizes clock cells within the suprachiasmatic circadian clock. Neuron, 25(1), 123-128. http://doi.org/10.1016/S0896-6273(00)80876-4

Liu, C., Weaver, D. R., Jin, X., Shearman, L. P., Pieschl, R. L., Gribkoff, V. K., \& Reppert, S. M. (1997). Molecular dissection of two distinct actions of melatonin on the suprachiasmatic circadian clock. Neuron, 19, 91-102.

Liu, X., Hubbard, J. A., Fabes, R. A., \& Adam, J. B. (2006). Sleep disturbances and correlates of children with autism spectrum disorders. Child Psychiatry and Human Development, 37(2), 179-91. http://doi.org/10.1007/s10578-006-0028-3

Lopez-Molina, L., Conquet, F., Dubois-Dauphin, M., \& Schibler, U. (1997). The DBP gene is expressed according to a circadian rhythm in the suprachiasmatic nucleus and influences circadian behavior. The EMBO Journal, 16(22), 6762-6771. http://doi.org/10.1093/emboj/16.22.6762

Lord, C., Petkova, E., Hus, V., Gan, W., Lu, F., Martin, D. M., ... Risi, S. (2012). A multi-site study of the clinical diagnosis of different autism spectrum disorders. Archives of General Psychiatry, 69(3), 306-313. http://doi.org/10.1001/archgenpsychiatry.2011.148.

Lord, C., Risi, S., Lambrecht, L., Cook, E. H., Leventhal, B. L., DiLavore, P. C., ... Rutter, M. (2000). The Autism Diagnostic Observation Schedule-Generic: A standard measure of social and communication deficits associated with the spectrum of autism. Journal of Autism and Developmental Disorders, 30(3), 205-23. http://doi.org/10.1023/A:1005592401947

Lord, C., Rutter, M., Goode, S., Heemsbergen, J., Jordan, H., Mawhood, L., \& Schopler, E. (1989). Autism Diagnostic Observation Schedule: A standardized observation of communicative and social behavior. Journal of Autism and Developmental Disorders, 19(2), 185-212. Retrieved from http://www.ncbi.nlm.nih.gov/pubmed/2745388

Lord, C., Rutter, M., \& Le Couteur, A. (1994). Autism Diagnostic Interview-Revised: A revised version of a diagnostic interview for caregivers of individuals with possible pervasive developmental disorders. Journal of Autism and Developmental Disorders, 24(5), 659-85.

Luo, R., Sanders, S. J., Tian, Y., Voineagu, I., Huang, N., Chu, S. H., ... Geschwind, D. H. (2012). Genome-wide transcriptome profiling reveals the functional impact of rare de novo and recurrent CNVs in autism spectrum disorders. American Journal of Human Genetics, 91(1), 38-55. http://doi.org/10.1016/j.ajhg.2012.05.011

Luyster, F. S., Choi, J., Yeh, C.-H., Imes, C. C., Johansson, A. E. E., \& Chasens, E. R. (2015). Screening and evaluation tools for sleep disorders in older adults. Applied Nursing Research, 28(4), 334-340. http://doi.org/10.1016/j.apnr.2014.12.007

Maas, A. P. H. M., Didden, R., Korzilius, H., Braam, W., Collin, P., Smits, M. G., \& Curfs, L. M. G. 
(2011). Psychometric properties of a sleep questionnaire for use in individuals with intellectual disabilities. Research in Developmental Disabilities, 32(6), 2467-2479. http://doi.org/10.1016/j.ridd.2011.07.013

Malow, B. A., Connolly, H. V, Weiss, S. K., Halbower, A., Goldman, S., Hyman, S. L., ... Reynolds, A. M. (2016). The Pediatric Sleep Clinical Global Impressions Scale - A new tool to measure pediatric insomnia in autism spectrum disorders. Journal of Developmental and Behavioral Pediatrics, 37(5), 370-376. http://doi.org/10.1097/DBP.0000000000000307

Malow, B., Adkins, K., McGrew, S., Wang, L., Goldman, S. E., Fawkes, D., \& Burnette, C. (2012). Melatonin for sleep in children with autism: A controlled trial examining dose, tolerability, and outcomes. Journal of Autism and Developmental Disorders, 42(8), 1729-1737. http://doi.org/10.1007/s10803-011-1418-3.Melatonin

Malow, B., Adkins, K. W., Reynolds, A., Weiss, S. K., Loh, A., Fawkes, D., ... Clemons, T. (2014). Parent-based sleep education for children with autism spectrum disorders. Journal of Autism and Developmental Disorders, 44(1), 216-28. http://doi.org/10.1007/s10803-013-1866-z

Malow, B., Marzec, M., McGrew, S., Wang, L., Henderson, L., \& Stone, W. (2006). Characterizing sleep in children with autism spectrum disorders: A multidimensional approach. Sleep, 29(12), 15631571.

Markovich, A. N., Gendron, M. A., \& Corkum, P. V. (2015). Validating the Children's Sleep Habits Questionnaire against polysomnography and actigraphy in school-aged children. Frontiers in Psychiatry, 6, 1-10. http://doi.org/10.3389/fpsyt.2014.00188

Masumoto, K. H., Nagano, M., Takashima, N., Hayasaka, N., Hiyama, H., Matsumoto, S. I., ... Shigeyoshi, Y. (2006). Distinct localization of prokineticin 2 and prokineticin receptor 2 mRNAs in the rat suprachiasmatic nucleus. European Journal of Neuroscience, 23(11), 2959-2970. http://doi.org/10.1111/j.1460-9568.2006.04834.x

May, T., Cornish, K., Conduit, R., Rajaratnam, S. M. W., \& Rinehart, N. J. (2015). Sleep in highfunctioning children with autism: Longitudinal developmental change and associations with behavior problems. Behavioral Sleep Medicine, 13(1), 2-18. http://doi.org/10.1080/15402002.2013.829064

Mayes, S. D., \& Calhoun, S. L. (2009). Variables related to sleep problems in children with autism. Research in Autism Spectrum Disorders, 3, 931-941. http://doi.org/10.1016/j.rasd.2009.04.002

Mazurek, M. O., \& Petroski, G. F. (2015). Sleep problems in children with autism spectrum disorder: Examining the contributions of sensory over-responsivity and anxiety. Sleep Medicine, 16(2), 270279. http://doi.org/10.1016/j.sleep.2014.11.006

McCrimmon, A. W., \& Smith, A. D. (2012). Review of the Wechsler Abbreviated Scale of Intelligence, Second Edition (WASI-II). Journal of Psychoeducational Assessment, 31(3), 337-341. http://doi.org/10.1177/0734282912467756

McNemar, Q. (1947). Note on the sampling error of the difference between correlated proportions or percentages. Psychometrika, 12(2), 153-157. http://doi.org/10.1007/BF02295996

Melke, J., Goubran Botros, H., Chaste, P., Betancur, C., Nygren, G., Anckarsäter, H., ... the PARIS 
study. (2008). Abnormal melatonin synthesis in autism spectrum disorders. Molecular Psychiatry, 13, 90-98. http://doi.org/10.1038/sj.mp.4002016

Miano, S., Bruni, O., Elia, M., Trovato, A., Smerieri, A., Verrillo, E., ... Ferri, R. (2007). Sleep in children with autistic spectrum disorder: A questionnaire and polysomnographic study. Sleep Medicine, 9(1), 64-70. http://doi.org/10.1016/j.sleep.2007.01.014

Mindell, J. A., Leichman, E. S., \& Composto, J. (2016). Development of infant and toddler sleep patterns: Real-world data from a mobile application. Journal of Sleep Research, 25, 508-516. http://doi.org/10.1111/jsr.12414

Mindell, J. A., \& Moore, M. (2014). Bedtime problems and night wakings. In S. H. Sheldon, R. Ferber, M. Kryder, \& D. Gozal (Eds.), Principles and Practice of Pediatric Sleep Medicine (2nd ed., pp. 105-109). St. Louis, MO: Elsevier Inc.

Mindell, J. A., Sadeh, A., Kohyama, J., \& How, T. H. (2010). Parental behaviors and sleep outcomes in infants and toddlers: A cross-cultural comparison. Sleep Medicine, 11(4), 393-9. http://doi.org/10.1016/j.sleep.2009.11.011

Mochizuki, T., Crocker, A., McCormack, S., Yanagisawa, M., Sakurai, T., \& Scammell, T. E. (2004). Behavioral state instability in orexin knock-out mice. Journal of Neuroscience, 24(28), 6291-6300. http://doi.org/10.1523/JNEUROSCI.0586-04.2004

Montgomery, S. B., Sammeth, M., Gutierrez-Arcelus, M., Radoslaw, P. L., Ingle, C., Nisbett, J., ... Dermitzakis, E. T. (2013). Transcriptome genetics using second generation sequencing in a Caucasian population. Nature, 464(7289), 1-16. http://doi.org/10.1038/nature08903

Moore, R. Y. (1996). Entrainment pathways and the functional organization of the circadian system. Progress in Brain Research, 111, 103-119.

Moore, R. Y., Speh, J. C., \& Leak, R. K. (2002). Suprachiasmatic nucleus organization. Cell and Tissue Research, 309(1), 89-98. http://doi.org/10.1007/s00441-002-0575-2

Moreira, F., Pedrazzoli, M., Dos Santos Coelho, F., Pradella-Hallinan, M., Lopes da Conceição, M., Pereira Peregrino, A., ... Tufik, S. (2005). Clock gene polymorphisms and narcolepsy in positive and negative HLA-DQB1*0602 patients. Brain Research: Molecular Brain Research, 140(1-2), $150-154$.

Nakashima, A., Kawamoto, T., Honda, K. K., Ueshima, T., Noshiro, M., Iwata, T., ... Kato, Y. (2008). DEC1 modulates the circadian phase of clock gene expression. Molecular and Cellular Biology, 28(12), 4080-4092. http://doi.org/10.1128/MCB.02168-07

National Center for Biotechnology Information. (2014a). ARNTL aryl hydrocarbon receptor nuclear translocator-like [Homo sapiens (human)]. Retrieved from http://www.ncbi.nlm.nih.gov/gene/406

National Center for Biotechnology Information. (2014b). ASMT acetylserotonin O-methyltransferase [ Homo sapiens (human) ]. Retrieved from http://www.ncbi.nlm.nih.gov/gene/438

National Center for Biotechnology Information. (2014a). CLOCK clock circadian regulator [Homo sapiens (human)]. Retrieved from http://www.ncbi.nlm.nih.gov/gene/9575\#reference-sequences 
National Center for Biotechnology Information. (2014). CSNK1D casein kinase 1, delta [ Homo sapiens (human) ]. Retrieved from http://www.ncbi.nlm.nih.gov/gene/1453

National Center for Biotechnology Information. (2014b). DDC dopa decarboxylase (aromatic L-amino acid decarboxylase) [ Homo sapiens (human) ]. Retrieved from http://www.ncbi.nlm.nih.gov/gene/1644

Nicholas, B., Rudrasingham, V., Nash, S., Kirov, G., Owen, M. J., \& Wimpory, D. C. (2007). Association of Per1 and Npas2 with autistic disorder: Support for the clock genes/social timing hypothesis. Molecular Psychiatry, 12(6), 581-92. http://doi.org/10.1038/sj.mp.4001953

Nieto, P. S., Revelli, J. A., Garbarino-Pico, E., Condat, C. A., Guido, M. E., \& Tamarit, F. A. (2015). Effects of different PER translational kinetics on the dynamics of a core circadian clock model. Plos One, 10(1), e0115067. http://doi.org/10.1371/journal.pone.0115067

Nir, I., Meir, D., Zilber, N., Knobler, H., \& Hadjez, J. (1995). Brief report: Circadian melatonin, thyroidstimulating hormone, prolactin, and cortisol levels in serum of young adults with autism. Journal of Autism and Developmental Disorders, 25(6), 641-654.

O’Connell, K. S., Mcgregor, N. W., Lochner, C., Emsley, R., \& Warnich, L. (2018). The genetic architecture of schizophrenia , bipolar disorder, obsessive- compulsive disorder and autism spectrum disorder. Molecular and Cellular Neuroscience, 88, 300-307. http://doi.org/10.1016/j.mcn.2018.02.010

O’Hara, B. F., Andretic, R., Heller, H. C., Carter, D. B., \& Kilduff, T. S. (1995). GABAA, GABAC, and NMDA receptor subunit expression in the suprachiasmatic nucleus and other brain regions. Molecular Brain Research, 28(2), 239-250. http://doi.org/10.1016/0169-328X(94)00212-W

Oliveras-Rentas, R. E., Kenworthy, L., Roberson, R. B., Martin, A., \& Wallace, G. L. (2013). WISC-IV profile in high-functioning autism spectrum disorders: Impaired processing speed is associated with increased autism communication symptoms and decreased adaptive communication abilities. Journal of Autism and Developmental Disorders, 42(5), 655-664. http://doi.org/10.1007/s10803011-1289-7.WISC-IV

Ornitz, E., Ritvo, E., Brown, M., La Franchi, S., Parmelee, T., \& Walter, R. (1969). The EEG and rapid eye movements during REM sleep in normal and autistic children. Electroencephalography and Clinical Neurophysiology, 26(2), 167-175.

Owens, J., Spirito, A., \& McGuinn, M. (2000). The Children’s Sleep Habits Questionnaire (CSHQ): Psychometric properties of a survey instrument for school-aged children. Sleep, 23(8), 1043-51.

Pagan, C., Delorme, R., Callebert, J., Goubran-Botros, H., Amsellem, F., Drouot, X., ... Launay, J.-M. (2014). The serotonin-N-acetylserotonin-melatonin pathway as a biomarker for autism spectrum disorders. Translational Psychiatry, 4(11), e479. http://doi.org/10.1038/tp.2014.120

Pagan, C., Goubran-Botros, H., Delorme, R., Benabou, M., Lemière, N., Murray, K., ... Bourgeron, T. (2017). Disruption of melatonin synthesis is associated with impaired 14-3-3 and miR-451 levels in patients with autism spectrum disorders. Scientific Reports, 7(1), 2096. http://doi.org/10.1038/s41598-017-02152-x

Pandi-Perumal, S. R., Srinivasan, V., Spence, D. W., \& Cardinali, D. P. (2007). Role of the melatonin 
system in the control of sleep: Therapeutic implications. CNS Drugs, 21(12), 995-1018. http://doi.org/10.2165/00023210-200721120-00004

Parks, G. S., Olivas, N. D., Ikrar, T., Sanathara, N. M., Wang, L., Wang, Z., ... Xu, X. (2014). Histamine inhibits the melanin-concentrating hormone system: Implications for sleep and arousal. The Journal of Physiology, 0, 1-14. http://doi.org/10.1113/jphysiol.2013.268771

Popa, D., El Yacoubi, M., Vaugeois, J.-M., Hamon, M., \& Adrien, J. (2006). Homeostatic regulation of sleep in a genetic model of depression in the mouse: Effects of muscarinic and 5-HT1A receptor activation. Neuropsychopharmacology, 31(8), 1637-46. http://doi.org/10.1038/sj.npp.1300948

Porkka-Heiskanen, T., Strecker, R. E., Thakkar, M., Bjorkum, A. A., Greene, R. W., \& McCarley, R. W. (1997). Adenosine: A mediator of the sleep-inducing effects of prolonged wakefulness. Science, 276(5316), 1265-1268. http://doi.org/10.1126/science.276.5316.1265

PsychCorp. (2016). Differential Ability Scales-II (DAS-II). Retrieved February 27, 2016, from http://www.pearsonclinical.com/education/products/100000468/differential-ability-scales-ii-dasii.html\#tab-scoring

Purcell, S., Cherny, S., \& Sham, P. (2003). Genetic Power Calculator: design of linkage and association genetic mapping studies of complex traits. Bioinformatics, 19(1), 149-150.

R Core Team. (2016). R: A language and environment for statistical computing. Vienna, Austria: R Foundation for Statistical Computing. Retrieved from https://www.r-project.org/

Ramasamy, A., Trabzuni, D., Guelfi, S., Varghese, V., American Brain Expression Consortium, Coin, L., ... Weale, M. E. (2014). Genetic variability in the regulation of gene expression in ten regions of the human brain. Nature Neuroscience, 17(10), 1418-1428. http://doi.org/10.1038/nn.3801

Ren, Z., \& Qiu, A. (2014). Sleep-related breathing disorder is associated with hyperactivity in preschoolers. Singapore Medical Journal, 55(5), 257-260. http://doi.org/10.11622/smedj.2014068

Riquelme, I., Hatem, S. M., \& Montoya, P. (2016). Abnormal pressure pain, touch sensitivity, proprioception , and manual dexterity in children with autism spectrum disorders. Neural Plasticity, 2016, 1-9. http://doi.org/10.1155/2016/1723401

Roland, P. S., Rosenfeld, R. M., Brooks, L. J., Friedman, N. R., Jones, J., Kim, T. W., .. Robertson, P. (2011). Clinical practice guideline: Polysomnography for sleep-disordered breathing prior to tonsillectomy in children. Otolaryngology--Head and Neck Surgery, 145, S1-S15. http://doi.org/10.1177/0194599811409837

Rosen, G. (2014). Disorders of arousal. In S. H. Sheldon, R. Ferber, M. Kryger, \& D. Gozal (Eds.), Principles and Practice of Pediatric Sleep Medicine (2nd ed., pp. 313-320). St. Louis, MO: Elsevier Inc.

Rossignol, D. a, \& Frye, R. E. (2011). Melatonin in autism spectrum disorders: a systematic review and meta-analysis. Developmental Medicine and Child Neurology, 53(9), 783-92. http://doi.org/10.1111/j.1469-8749.2011.03980.x

Sadeh, A., Mindell, J., \& Rivera, L. (2011). “'My child has a sleep problem "”: A cross-cultural comparison of parental definitions. Sleep Medicine, 12(5), 478-482. 
http://doi.org/10.1016/j.sleep.2010.10.008

Sanders, S. J., He, X., Willsey, A. J., Ercan-Sencicek, A. G., Kaitlin, E., Cicek, A. E., ... Somer, L. (2015). Insights into autism spectrum disorder genomic architecture and biology from 71 risk loci. Neuron, 87(6), 1215-1233. http://doi.org/10.1016/j.neuron.2015.09.016.Insights

Sangoram, A. M., Saez, L., Antoch, M. P., Gekakis, N., Staknis, D., Whiteley, A., ... Takahashi, J. S. (1998). Mammalian circadian autoregulatory loop: A timeless ortholog and mPer1 interact and negatively regulate CLOCK-BMAL1-induced transcription. Neuron, 21(5), 1101-1113. http://doi.org/10.1016/S0896-6273(00)80627-3

Sato, F., Kawamoto, T., Fujimoto, K., Noshiro, M., Honda, K. K., Honma, S., ... Kato, Y. (2004). Functional analysis of the basic helix-loop-helix transcription factor DEC1 in circadian regulation. Interaction with BMAL1. European Journal of Biochemistry / FEBS, 271(22), 4409-19. http://doi.org/10.1111/j.1432-1033.2004.04379.x

Schadt, E. E., Molony, C., Chudin, E., Hao, K., Yang, X., Lum, P. Y., ... Ulrich, R. (2008). Mapping the genetic architecture of gene expression in human liver. PLoS Biology, 6(5), e107. http://doi.org/10.1371/journal.pbio.0060107

Scheer, F. A. J. L., Ter Horst, G. J., Van der Vliet, J., \& Buijs, R. M. (2001). Physiological and anatomic evidence for regulation of the heart by suprachiasmatic nucleus in rats. American Journal of Physiology-Heart, 280, H1391-H1399.

Schreck, K., Mulick, J. A., \& Smith, A. F. (2004). Sleep problems as possible predictors of intensified symptoms of autism. Research in Developmental Disabilities, 25(1), 57-66. http://doi.org/10.1016/j.ridd.2003.04.007

Schwender, H., Li, Q., Berger, P., Neumann, C., Taub, M., \& Ruczinski, I. (2015). trio: Testing of SNPs and SNP interactions in case-parent trio studies.

Schwichtenberg, A. J., Young, G. S., Hutman, T., Iosif, A.-M., Sigman, M., Rogers, S. J., \& Ozonoff, S. (2013). Behavior and sleep problems in children with a family history of autism. Autism Research, 6(3), 169-76. http://doi.org/10.1002/aur.1278

Shanware, N. P., Hutchinson, J. A., Kim, S. H., Zhan, L., Bowler, M. J., \& Tibbetts, R. S. (2011). Casein kinase 1-dependent phosphorylation of familial advanced sleep phase syndrome-associated residues controls PERIOD 2 stability. The Journal of Biological Chemistry, 286(14), 12766-74. http://doi.org/10.1074/jbc.M111.224014

Sheldon, S. (2014). Development of sleep in infants and children. In S. Sheldon, R. Ferber, M. H. Kryger, \& D. Gozal (Eds.), Principles and Practice of Pediatric Sleep Medicine (2nd ed., pp. 17-23). St. Louis, MO: Elsevier Inc.

Sheldon, S. H. (2014). Development of sleep in infants and children. In Principles and Practice of Pediatric Sleep Medicine. (2nd ed., pp. 17-23). St. Louis, MO: Elsevier.

Short, M. A., Arora, T., Gradisar, M., Taheri, S., \& Carskadon, M. A. (2017). How many sleep diary entries are needed to reliably estimate adolescent sleep? Sleep, 40(3), 1-10.

Short, M., Gradisar, M., Lack, L., Wright, H., \& Carskadon, M. (2012). The discrepancy between 
actigraphic and sleep diary measures of sleep in adolescents. Sleep Medicine, 13(4), 378-384.

Sikora, D. M., Johnson, K., Clemons, T., \& Katz, T. (2012). The relationship between sleep problems and daytime behavior in children of different ages with autism spectrum disorders. Pediatrics, 130, S83S90. http://doi.org/10.1542/peds.2012-0900F

Simonds, J. F., \& Parraga, H. (1982). Prevalence of sleep disorders and sleep behaviors in children and adolescents. Journal of the American Academy of Child Psychiatry, 21(4), 383-8.

Simons Foundation Autism Research Initiative. (2014). SFARI Base / SSC / Simons VIP researcher welcome packet. New York. Retrieved from https://sfari.org/resources/sfari-base

Souders, M. C., Mason, T. B. A., Valladares, O., Bucan, M., Levy, S. E., Mandell, D. S., ... Pinto-Martin, J. (2009). Sleep behaviors and sleep quality in children with autism spectrum disorders. Sleep, 32(12), 1566-78. Retrieved from https://www.ncbi.nlm.nih.gov/pmc/articles/PMC2786040/

Sparrow, S., Balla, D., Cicchetti, D., Harrison, P., \& Doll, E. (1984). Vineland Adaptive Behavior Scales. Circle Pines, MN: American Guidance Service.

Stenberg, D., Litonius, E., Halldner, L., Johansson, B., Fredholm, B. B., \& Porkka-Heiskanen, T. (2003). Sleep and its homeostatic regulation in mice lacking the adenosine A1 receptor. Journal of Sleep Research, 12(4), 283-90. Retrieved from http://www.ncbi.nlm.nih.gov/pubmed/14633239

Stickgold, R. (2005). Sleep-dependent memory consolidation. Nature, 437, 1272-1278.

Stranger, B. E., Nica, A. C., Forrest, M. S., Dimas, A., Bird, C. P., Beazley, C., ... Dermitzakis, E. T. (2009). Population genomics of human gene expression. Nature Genetics, 39(10), 1217-1224. http://doi.org/10.1038/ng2142

Su, M. S., Li, A. M., So, H. K., Au, C. ., Ho, C., \& Wing, Y. K. (2011). Nocturnal enuresis in children: Prevalence, correlates, and relationship with obstructive sleep apnea. The Journal of Pediatrics, 159(2), 238-242.e1. http://doi.org/10.1016/j.jpeds.2011.01.036

Sumi-Ichinose, C., Ichinose, H., Takahashi, E., Hori, T., \& Nagatsu, T. (1992). Molecular cloning of genomic DNA and chromosomal assignment of the gene for human aromatic L-amino acid decarboxylase, the enzyme for catecholamine and serotonin biosynthesis. Biochemistry, 31(8), 2229-38. Retrieved from http://www.ncbi.nlm.nih.gov/pubmed/1540578

Tabachnick, B., \& Fidell, L. (2013a). Cleaning up your act: Screening data prior to analysis. In Using Multivariate Statistics (6th ed., pp. 60-116). Boston, MA: Pearson.

Tabachnick, B., \& Fidell, L. (2013b). Principal components and factor analysis. In Using Multivariate Statistics (6th ed., pp. 612-680). Boston, MA: Pearson.

Takano, A., Uchiyama, M., Kajimura, N., Mishima, K., Inoue, Y., Kamei, Y., ... Ebisawa, T. (2004). A missense variation in human casein kinase I epsilon gene that induces functional alteration and shows an inverse association with circadian rhythm sleep disorders. Neuropsychopharmacology, 29(10), 1901-9. http://doi.org/10.1038/sj.npp.1300503

Tanguay, P. E., Ornitz, E. M., Forsythe, A. B., \& Ritvo, E. R. (1976). Rapid eye movement (REM) activity in normal and autistic children during REM sleep. Journal of Autism and Childhood 
Schizophrenia, 6(3), 275-88. Retrieved from http://www.ncbi.nlm.nih.gov/pubmed/186448

Tarokh, L., \& Carskadon, M. A. (2010). Developmental changes in the human sleep EEG during early adolescence. Sleep, 33(6), 801-809.

Tasse, M. J., Luckasson, R., \& Nygren, M. (2013). AAIDD proposed recommendations for ICD-11 and the condition previously known as mental retardation. Intellectual and Developmental Disabilities, 51(2), 127-131.

Taylor, M. A., Schreck, K. A., \& Mulick, J. A. (2012). Sleep disruption as a correlate to cognitive and adaptive behavior problems in autism spectrum disorders. Research in Developmental Disabilities, 33(5), 1408-17. http://doi.org/10.1016/j.ridd.2012.03.013

Thakkar, M. M. (2011). Histamine in the regulation of wakefulness. Sleep Medicine Reviews, 15(1), 6574. http://doi.org/10.1016/j.smrv.2010.06.004

The Autism Spectrum Disorders Working Group of The Psychiatric Genomics Consortium. (2017). Metaanalysis of GWAS of over 16,000 individuals with autism spectrum disorder highlights a novel locus at 10q24.32 and a significant overlap with schizophrenia. Molecular Autism, 8(21), 1-17. http://doi.org/10.1186/s13229-017-0137-9

The GTEx Consortium. (2015). Human genomics. The Genotype-Tissue Expression (GTEx) pilot analysis: Multitissue gene regulation in humans. Science, 348, 648-660. http://doi.org/10.1126/science.1262110

The Psychological Corporation. (1999). WASI Manual. San Antonio, TX: Harcourt Brace \& Company.

Tietze, A. L., Zernikow, B., Michel, E., \& Blankenburg, M. (2014). Sleep disturbances in children, adolescents, and young adults with severe psychomotor impairment: Impact on parental quality of life and sleep. Developmental Medicine and Child Neurology, 56(12), 1187-1193. http://doi.org/10.1111/dmcn.12530

Tononi, G., \& Cirelli, C. (2006). Sleep function and synaptic honeostasis. Sleep Medicine Reviews, 10, 49-62.

Tordjman, S., Anderson, G. M., Pichard, N., Charbuy, H., \& Touitou, Y. (2005). Nocturnal excretion of 6-sulphatoxymelatonin in children and adolescents with autistic disorder. Biological Psychiatry, 57(2), 134-8. http://doi.org/10.1016/j.biopsych.2004.11.003

UniProtKB. (2014a). O15534 (PER1_HUMAN). Retrieved from http://www.uniprot.org/uniprot/O15534\#section_comments

UniProtKB. (2014b). Q16526 (CRY1_HUMAN). Retrieved from http://www.uniprot.org/uniprot/Q16526

Utge, S. J., Soronen, P., Loukola, A., Kronholm, E., Ollila, H. M., Pirkola, S., ... Paunio, T. (2010). Systematic analysis of circadian genes in a population-based sample reveals association of TIMELESS with depression and sleep disturbance. PloS One, 5(2), e9259. http://doi.org/10.1371/journal.pone.0009259

Vázquez-Palacios, G., Retana-Márquez, S., Bonilla-Jaime, H., \& Velázquez-Moctezuma, J. (2001). Further definition of the effect of corticosterone on the sleep-wake pattern in the male rat. 
Pharmacology, Biochemistry, and Behavior, 70(2-3), 305-10. Retrieved from http://www.ncbi.nlm.nih.gov/pubmed/11701201

Veatch, O. J., Maxwell-Horn, A. C., \& Malow, B. A. (2015). Sleep in autism spectrum disorders. Current Sleep Medicine Reports, 1(2), 131-140. http://doi.org/10.1007/s40675-015-0012-1

Veatch, O. J., Pendergast, J. S., Allen, M. J., Leu, R. M., Hirschie Johnson, C., Elsea, S. H., \& Malow, B. A. (2015). Genetic variation in melatonin pathway enzymes in children with autism spectrum disorder and comorbid sleep onset delay. Journal of Autism \& Developmental Disorders, 45(1), 100-110. http://doi.org/10.1007/s10803-014-2197-4

Veatch, O. J., Reynolds, A., Katz, T., Weiss, S. K., Loh, A., Wang, L., \& Malow, B. A. (2016). Sleep in children with autism spectrum disorders: How are measures of parent report and actigraphy related and affected by sleep education? Behavioral Sleep Medicine, 14(6), 665-676. http://doi.org/10.1080/15402002.2015.1065408

Veatch, O. J., Sutcliffe, J. S., Warren, Z. E., Keenan, B. T., Potter, M. H., \& Malow, B. A. (2017). Shorter sleep duration is associated with social impairment and comorbidities in ASD. Autism Research, 0, 1-18. http://doi.org/10.1002/aur.1765

Viscidi, E. W., Triche, E. W., Pescosolido, M. F., Mclean, R. L., Joseph, R. M., Spence, S. J., \& Morrow, E. M. (2013). Clinical characteristics of children with autism spectrum disorder and co-occurring epilepsy. PloS One, 8(7), 1-11. http://doi.org/10.1371/journal.pone.0067797

Walker, G., \& Shostak, J. (2010). McNemar's test. In Common Statistical Methods for Clinical Research with SAS® Examples (3rd ed., pp. 325-335). Cary, NC: SAS Institute, Inc.

Wang, G. Y., Lee, C. G. L., \& Lee, E. J. D. (2004). Genetic variability of arylalkylamine-N-acetyltransferase (AA-NAT) gene and human sleep/wake pattern. Chronobiology International, 21(2), 229-237. http://doi.org/10.1081/CBI-120037822

Wang, L., Li, J., Ruan, Y., Lu, T., Liu, C., Jia, M., ... Zhang, D. (2013). Sequencing ASMT identifies rare mutations in Chinese Han patients with autism. PloS One, 8(1), e53727. http://doi.org/10.1371/journal.pone.0053727

Webb, S. J., Garrison, M. M., Bernier, R., Mcclintic, A. M., King, B. H., \& Mourad, P. D. (2016). Severity of ASD symptoms and their correlation with the presence of copy number variations and exposure to first trimester ultrasound. Autism Research, 10(3), 472-484. http://doi.org/10.1002/aur.1690

Weschler, D. (2003). WISC-IV administration and scoring manual: Wechsler Intelligence Scale for Children- Fourth Edition. San Antonio, TX: PsychCorp.

Westra, H.-J., Peters, M. J., Esko, T., Yaghootkar, H., Schurmann, C., Kettunen, J., ... Franke, L. (2013). Systematic identification of trans-eQTLs as putative drivers of known disease associations. Nature Genetics, 45(10), 1238-1243. http://doi.org/10.1038/ng.2756

Wiggs, L., \& Stores, G. (1996). Severe sleep disturbance and daytime challenging behaviour in children with severe learning disabilities. Journal of Intellectual Disability Research, 40(6), 518-528. http://doi.org/10.1046/j.1365-2788.1996.799799.x 
Wiggs, L., \& Stores, G. (2004). Sleep patterns and sleep disorders in children with autistic spectrum disorders: Insights using parent report and actigraphy. Developmental Medicine and Child Neurology, 46(6), 372-80. Retrieved from http://www.ncbi.nlm.nih.gov/pubmed/15174528

Williams, K., Brignell, A., Prior, M., Bartak, L., \& Roberts, J. (2015). Regression in autism spectrum disorders. Journal of Paediatrics and Child Health, 51(1), 61-64. http://doi.org/10.1111/jpc.12805

Williams, P. E., Weiss, L. G., \& Rolfhus, E. L. (2003). WISC-IV technical report \#1: Theoretical model and test blueprint. San Antonio, TX: The Psychological Corporation.

Winsky-Sommerer, R. (2009). Role of GABAA receptors in the physiology and pharmacology of sleep. The European Journal of Neuroscience, 29(9), 1779-94. http://doi.org/10.1111/j.14609568.2009.06716.x

Wirojanan, J., Jacquemont, S., Diaz, R., Bacalman, S., Anders, T. F., Hagerman, R. J., \& Goodlin-Jones, B. L. (2009). The efficacy of melatonin for sleep problems in children with autism, fragile X syndrome, or autism and fragile X syndrome. Journal of Clinical Sleep Medicine : JCSM : Official Publication of the American Academy of Sleep Medicine, 5(2), 145-150. http://doi.org/10.1038/ejhg.2008.107

World Health Organization. (1996). ICD-10 guide for mental retardation. Geneva, Switzerland: World Health Organization. Retrieved from http://www.who.int/whr/2001/chapter2/en/index4.html

Wright, B., Sims, D., Smart, S., Alwazeer, A., Alderson-Day, B., Allgar, V., ... Miles, J. (2011). Melatonin versus placebo in children with autism spectrum conditions and severe sleep problems not amenable to behaviour management strategies: a randomised controlled crossover trial. Journal of Autism and Developmental Disorders, 41(2), 175-84. http://doi.org/10.1007/s10803-010-1036-5

Yamajuku, D., Shibata, Y., Kitazawa, M., Katakura, T., Urata, H., Kojima, T., ... Hashimoto, S. (2011). Cellular DBP and E4BP4 proteins are critical for determining the period length of the circadian oscillator. FEBS Letters, 585(14), 2217-2222. http://doi.org/10.1016/j.febslet.2011.05.038

Yang, Z., Matsumoto, A., Nakayama, K., Jimbo, E. F., Kojima, K., Nagata, K., ... Yamagata, T. (2016). Circadian-relevant genes are highly polymorphic in autism spectrum disorder patients. Brain and Development, 38(1), 91-99. http://doi.org/10.1016/j.braindev.2015.04.006

Yeung, C. K., Sreedhar, B., Sihoe, J. D. Y., Sit, F. K. Y., \& Lau, J. (2006). Differences in characteristics of nocturnal enuresis between children and adolescents: A critical appraisal from a large epidemiological study. BJU International, 97, 1069-1073. http://doi.org/10.1111/j.1464410X.2006.06074.x

Yoo, S. ., Gujar, N., Hu, P., Jolesz, F. A., \& Walker, M. P. (2007). The human emotional brain without sleep--A prefrontal amygdala disconnect. Current Biology, 17, R877-R878.

Zachor, D. A., \& Ben-Itzchak, E. (2016). Specific medical conditions are associated with unique behavioral profiles in autism spectrum disorders. Frontiers in Neuroscience, 10(410), 1-11. http://doi.org/10.3389/fnins.2016.00410

Zhang, X., Beaulieu, J.-M., Sotnikova, T. D., Gainetdinov, R. R., \& Caron, M. G. (2004). Tryptophan hydroxylase-2 controls brain serotonin synthesis. Science (New York, N.Y.), 305(5681), 217. http://doi.org/10.1126/science.1097540 
Zou, F., Chai, H. S., Younkin, C. S., Allen, M., Crook, J., Pankratz, V. S., ... Younkin, S. G. (2012). Brain expression genome-wide association study (eGWAS) sdentifies human disease-associated variants. PLoS Genetics, 8(6), e1002707. http://doi.org/10.1371/journal.pgen.1002707 